듬 SCHOOL of GRADUATE STUDIES

EAST TENNESSEE STATE UNIVERSITY
East Tennessee State University Digital Commons@ East Tennessee State University

$12-2014$

\title{
NICU Culture of Care for Infants with Neonatal Abstinence Syndrome: A Focused Ethnography
}

Monica M. Nelson

East Tennessee State University

Follow this and additional works at: https://dc.etsu.edu/etd

Part of the Maternal, Child Health and Neonatal Nursing Commons

\section{Recommended Citation}

Nelson, Monica M., "NICU Culture of Care for Infants with Neonatal Abstinence Syndrome: A Focused Ethnography" (2014).

Electronic Theses and Dissertations. Paper 2465. https://dc.etsu.edu/etd/2465

This Thesis - Open Access is brought to you for free and open access by the Student Works at Digital Commons @ East Tennessee State University. It has been accepted for inclusion in Electronic Theses and Dissertations by an authorized administrator of Digital Commons @ East Tennessee State

University. For more information, please contact digilib@etsu.edu. 
NICU Culture of Care for Infants with Neonatal Abstinence

Syndrome: A Focused Ethnography

\author{
A dissertation \\ presented to \\ the faculty of the Department of Nursing \\ East Tennessee State University
}

In partial fulfillment

of the requirements for the degree

Doctor of Philosophy in Nursing

by

Monica Marie Nelson

December 2014

Dr. Masoud Ghaffari, Chair

Dr. Sally Blowers, Committee Member

Dr. Sharon Loury, Committee Member

Dr. Jodie Murphy-Oikonen, Committee Member

Keywords: Neonatal Abstinence Syndrome, nursing care, nursing interventions, culture of care, focused ethnography 


\begin{abstract}
NICU Culture of Care for Infants with Neonatal Abstinence

Syndrome: A Focused Ethnography
\end{abstract}

by

Monica Marie Nelson

Purpose: The purpose of this ethnographic study was to describe the culture of care and nonpharmacological nursing interventions performed by NICU nurses for infants with NAS.

Background: Infants with NAS are increasingly being cared for in the inpatient hospital setting by NICU nurses. Interventions used for the care of premature and sick infants in the NICU may or may not be the exact interventions that should be used for the care of the fragile infant with NAS. Research studies on the nonpharmacological nursing care of infants with NAS encompass 5 main areas of practice: environment, adequate rest and sleep, feeding, assessment and evaluation using Finnegan scoring by nurses, and nurses as caregivers. Method: Roper and Shapira's (2000) framework for the analysis of ethnographic data was used for this research and included participant observation, individual interviews, and the examination to existing documents. Data analysis included: (a) coding for descriptive labels, (b) sorting to identify patterns, (c) identification of outliers or negative cases, (d) generalizing constructs and theories, and (e) memoing to note personal reflection and insights. Focused ethnography allows for the articulation of research questions before fieldwork while observing and describing a culture. Results: Five themes emerged from the data: learn the baby (routine care, comfort care, environment, adequate rest and sleep, feeding), core team relationships (support, interpersonal relationships), role satisfaction (nurturer or comforter, becoming an expert), grief, and making a difference (wonderful insanity, critical to them). Implications: The results of this were a 
description of the culture of care provided to infants with NAS by NICU nurses and provide general recommendations to the nurse caring for an infant with NAS in the areas of environment, adequate sleep and rest, feeding, and the role of the nurse. This study also has implications for future study of evidence-based research strategies to decrease withdrawal symptoms in infants with NAS. Research is needed in the areas of clinical practice guidelines to help the bedside nurse care for these infants using current research and evidence for practice. Nursing theory and nursing education both contribute to nursing research in how to better understand the culture of care provided by nurses. 


\section{DEDICATION}

I dedicate my dissertation work to my husband Mark and my children Michael, Matthew, and Meghan. My husband Mark has been my cheerleader throughout this process and without his love and support, I really could not have done this. Thank you sweet man. Frederick Buechner writes, "The place God calls you to is the place where your deep gladness and the world's deep hunger meet.” I am thankful and grateful that my family is that intersection of calling for me.

I also dedicate this work to my parents Roger and Patricia Long; their influence on me as a person in regards to being loving, caring, and committed to people above work has been the foundation of my life. Also a special dedication to my mom Patricia Carole Long, who was my first glimpse and example of a nurse. Also, to Mary Soja, my nursing instructor at Indiana University, who saw in me what I did not see in myself. My goal is to be this kind of teacher.

Lastly, I dedicate this work to the nurses who daily and fearlessly care for infants with NAS. Their depth of love, concern, and expertise in caring for these infants is like none other. They are fighting for those who cannot fight for themselves. I am in complete admiration of them and have been humbled and honored to conduct research with them. They are the heroes of this research. 


\section{ACKNOWLEDGEMENTS}

I wish to thank my committee members who were incredibly generous with their expertise and time. A very special thanks to Dr. Masoud Ghaffari, my committee chair, for his countless hours of reading, reflecting, providing feedback, and patience. Thank you also to Dr. Sally Blowers, Dr. Sharon Loury, and Dr. Jodie Murphy-Oikonen for serving on my committee. I have needed each of you to produce this scholarly work.

I would like to thank all of my teachers at East Tennessee State University and all of the preparation that they provided in coursework to allow me to arrive at this place. A huge "thank you” and hugs to all of the girls in my cohort, especially Kristen Hershey, who has become a lifelong friend. For me, it would have been impossible to do this work without a friend who "gets it.” Thank you also for my dear friend, Penny Neal, and her encouragement to me to even start a doctoral program. I am so glad that we work together. Thank you for your friendship.

Lastly, I would like to thank my Creator, who knows me and knows the challenges that I need to live. Thank You for opening doors, opportunities to keep learning, encouraging me to keep going, and for answering the daily prayer, "Please make me smart enough to do this.” 


\section{TABLE OF CONTENTS}

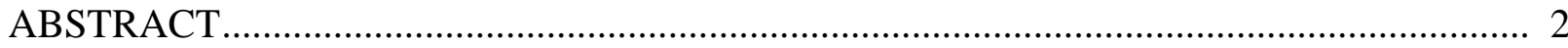

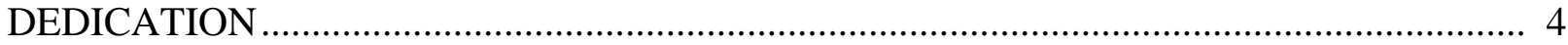

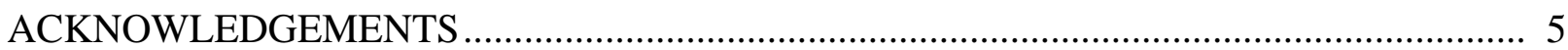

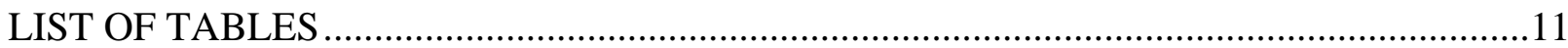

Chapter

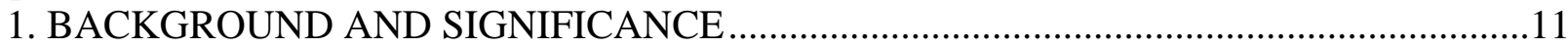

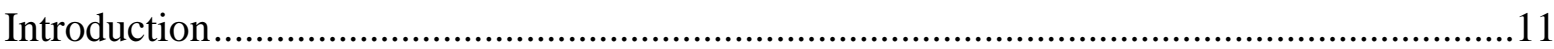

Research Problem, Research Questions, and Research Goals ........................................13

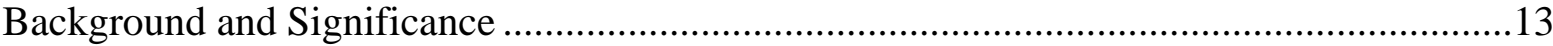

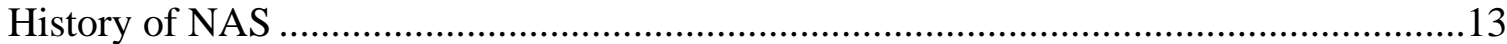

Known Drugs Taken by Mother ...............................................................................15

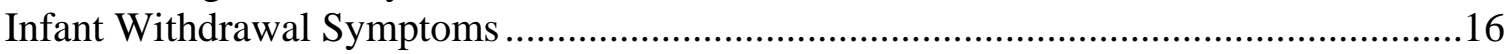

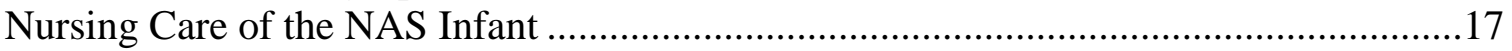

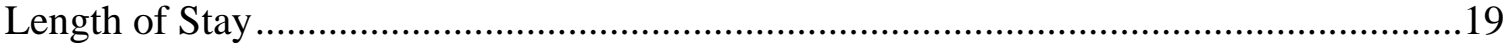

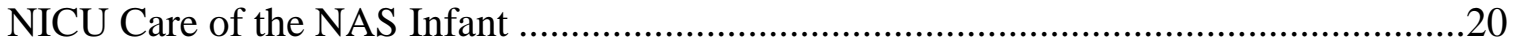

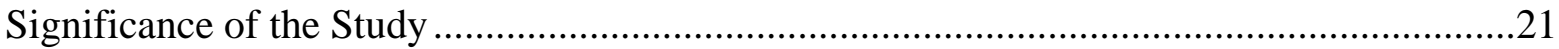

Philosophical Underpinnings ...............................................................................22

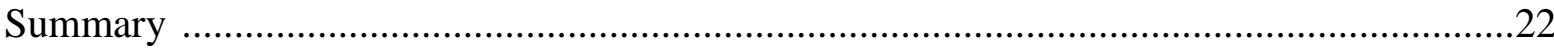

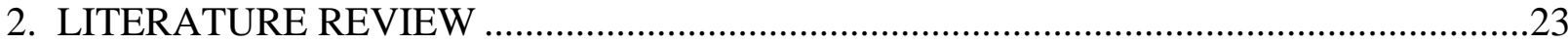

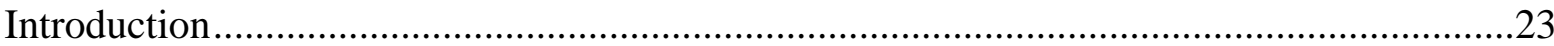

Gap Between Recommendations and Knowledge ..................................................25

Nonpharmacological Nursing Interventions ......................................................26

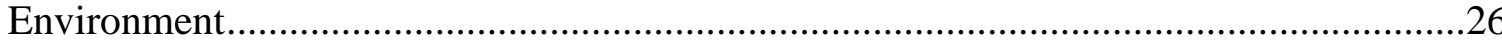

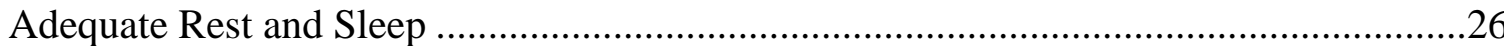

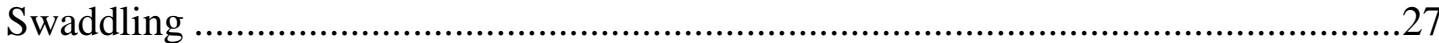

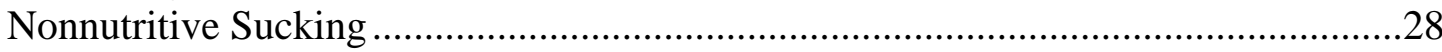

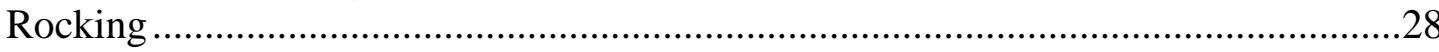

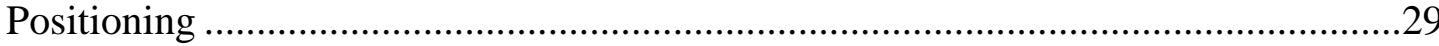

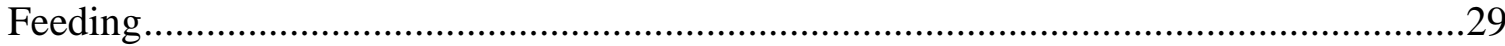

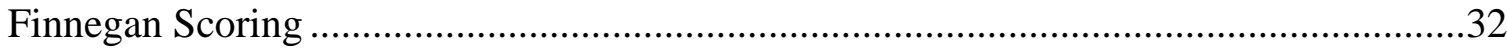

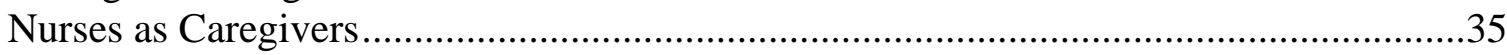

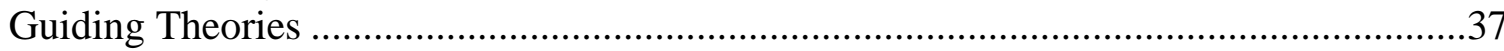

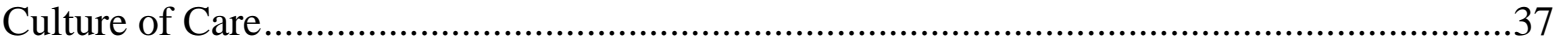

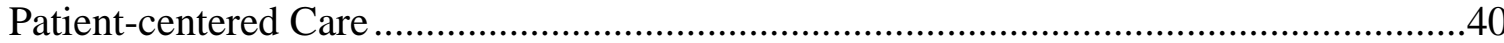

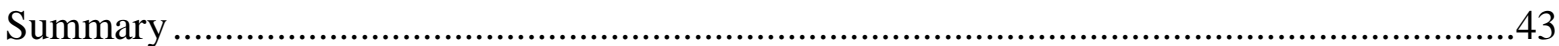

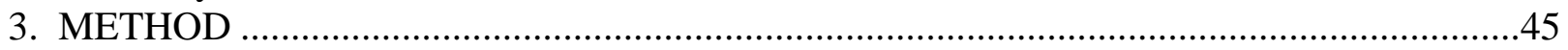

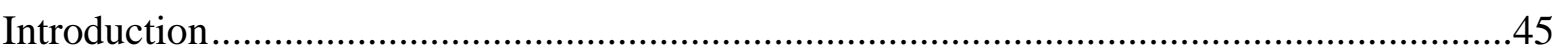

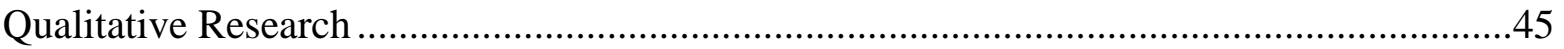

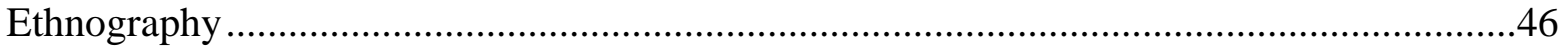

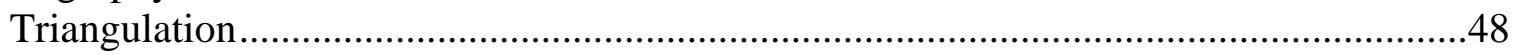

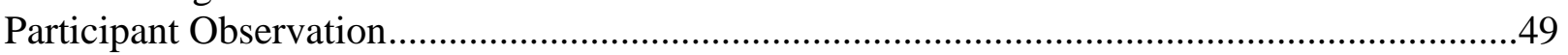

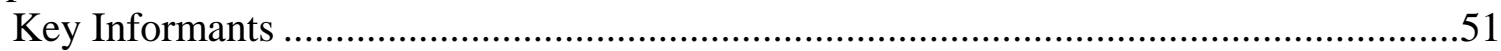

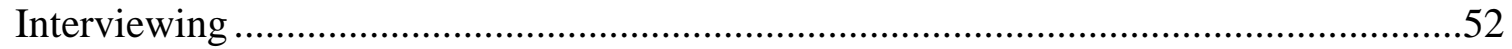

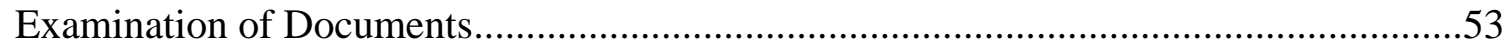




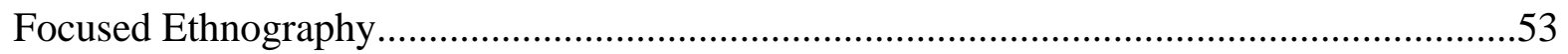

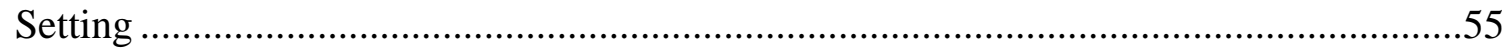

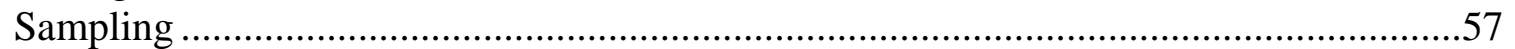

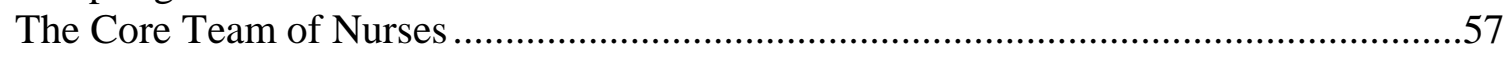

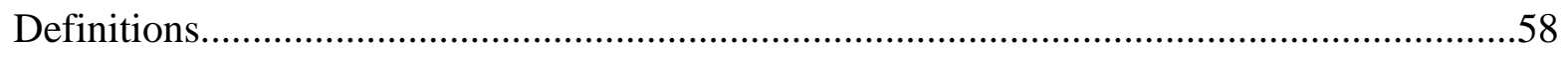

A Fictitious Example of a Typical Day in NICU ...........................................................60

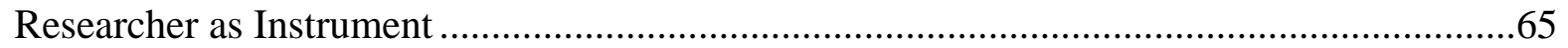

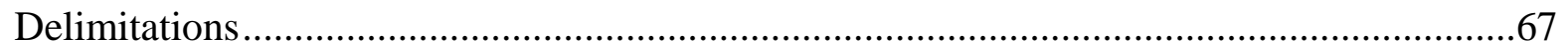

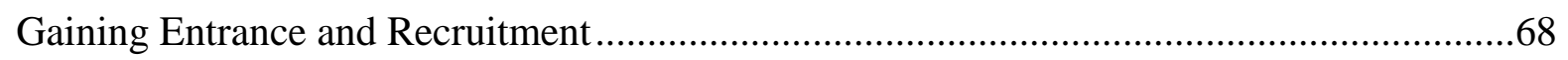

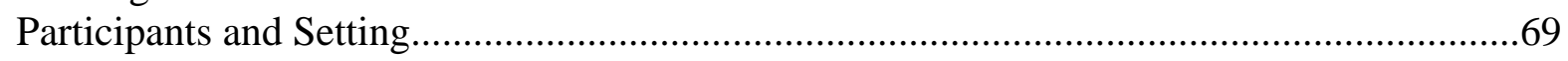

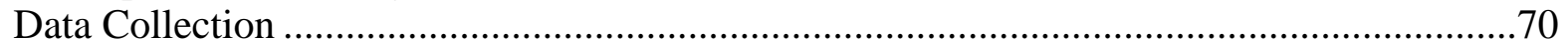

Participant Observation.........................................................................................70

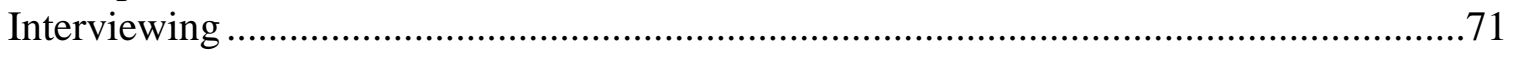

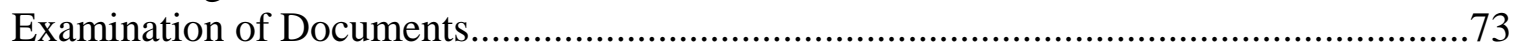

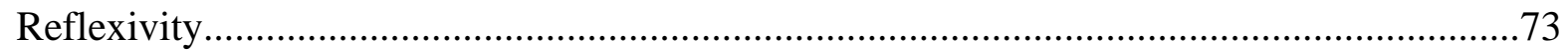

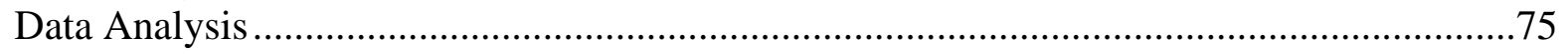

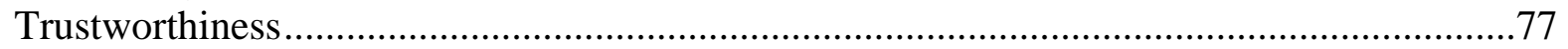

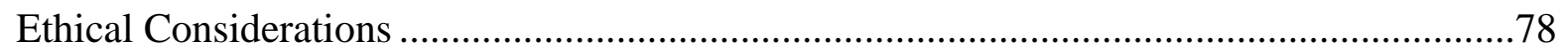

Protection of Human Subjects .........................................................................................8

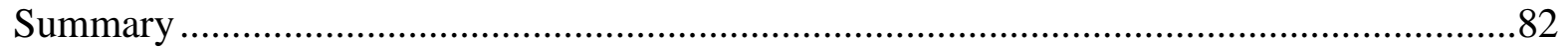

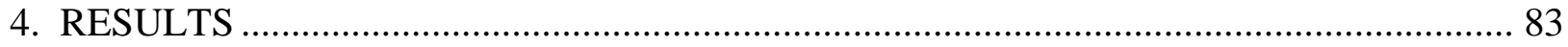

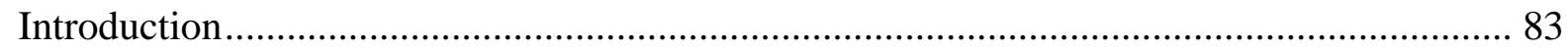

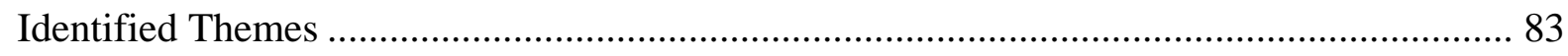

Theme 1: Learn the baby ……………………………............................................ 84

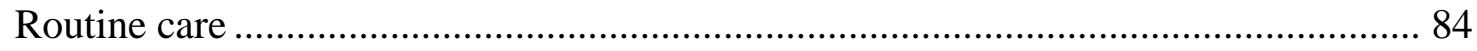

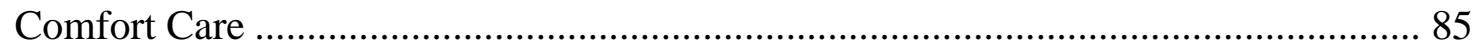

Environment (nursing intervention: environment: light, noise, touch)....................... 88

Culture of Care and Environment (observational results) ……………................... 90

Adequate Rest and Sleep (nursing intervention: adequate rest and sleep) ................... 92

Culture of Care and Adequate Rest and Sleep (observational results) ................... 93

Feeding (nursing intervention: feeding).......................................................................96

Culture of Care and Feeding (observational results) ................................................97

Theme 2: Core Team Relationships.............................................................................101

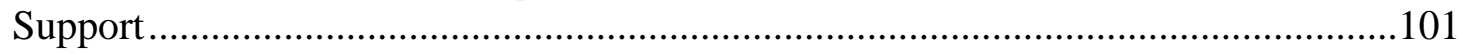

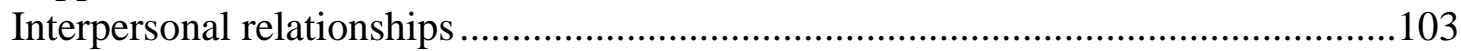

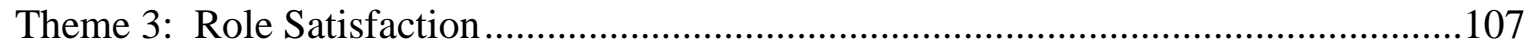

Nurturer or Comforter (nursing intervention: role of the nurse).................................107

Becoming an expert ...................................................................................................110

Culture of Care and the Role of the Nurse as Nurturer or Comforter

(observational results) ....................................................................................110

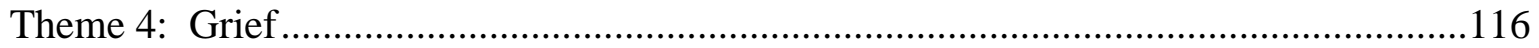

Theme 5: Making a Difference .......................................................................................119

"Wonderful insanity" (rewards and challenges) .........................................................119

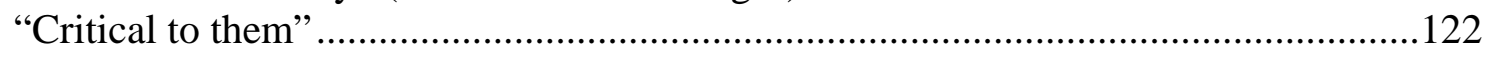

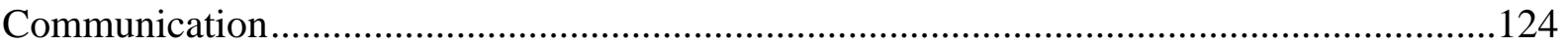

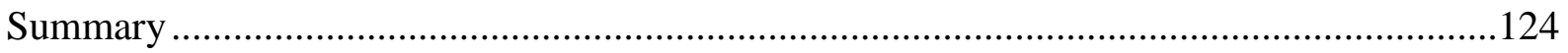




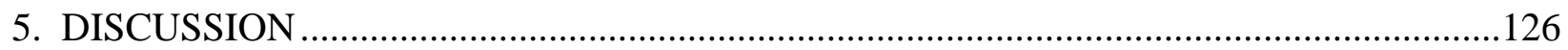

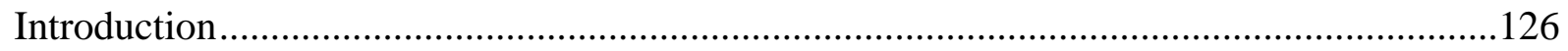

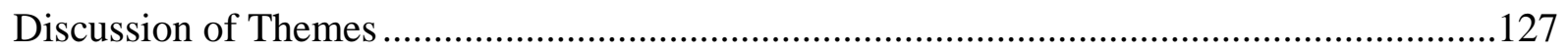

Theme 1: Learn the Baby (Subthemes: Routine Care, Comfort Care,

Environment, Adequate Rest and Sleep, Feeding) .....................................................127

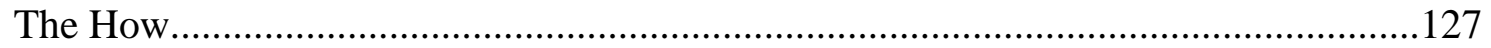

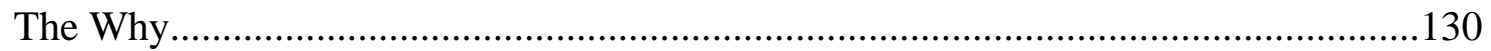

Care or Caring Component .............................................................................131

Recommendations for the Environment in Providing a Culture of Care for Infants with NAS....................................................................................131

Recommendations for Adequate Rest and Sleep in Proving a Culture of Care for Infants with NAS ..............................................................................132

Recommendations for Feeding in Providing a Culture of Care for Infants with NAS ...........................................................................................133

Theme 2: Core Team Relationships (Subthemes: Support, Interpersonal

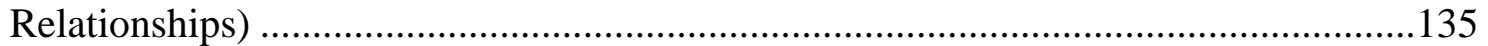

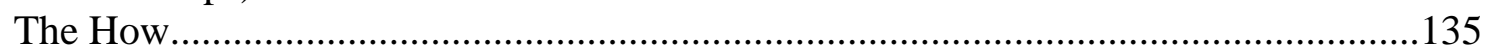

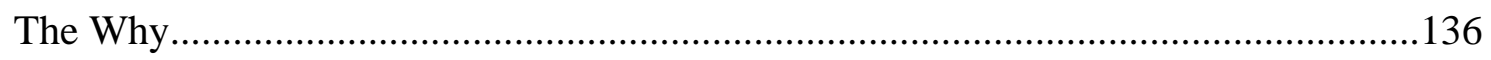

Care or Caring Component ......................................................................................137

Theme 3: Role Satisfaction (Subthemes: Nurturer or Comforter, Becoming an

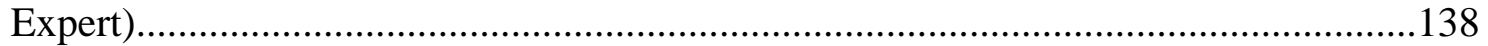

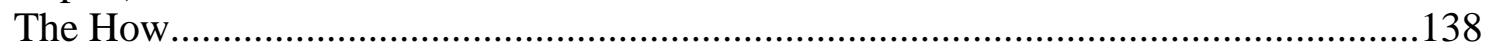

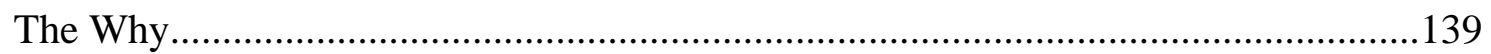

Care or Caring Component ......................................................................................140

Recommendations for the Nurturer or Comforter Role in Providing a Culture of Care for Infants with NAS..........................................................140

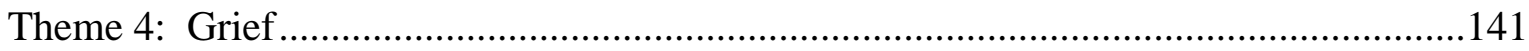

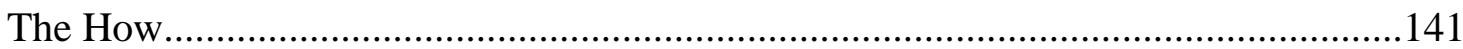

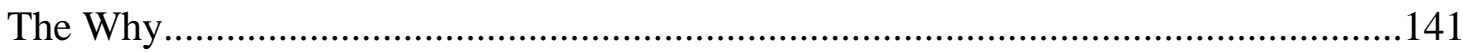

Care or Caring Component .......................................................................................142

Theme 5: Making a Difference (Subthemes: Wonderful Insanity, Critical to

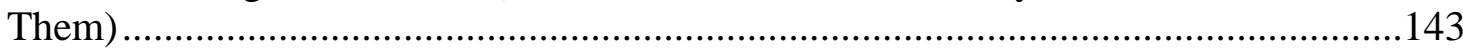

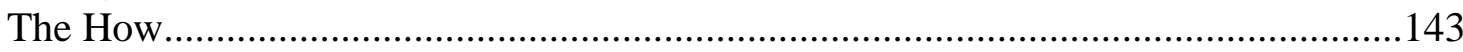

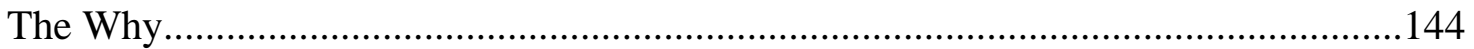

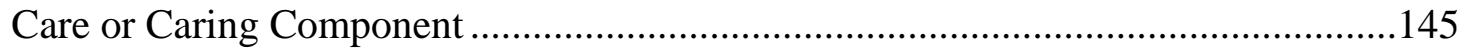

Discussion of Themes in Relationship to Research Questions .............................................145

The Meaning of the Culture of Care in NICU 3 ................................................................147

Conceptual Model: NICU Culture of Care for Infants with NAS ......................................150

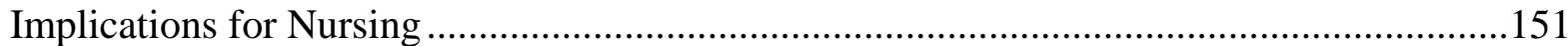

Implications for Nursing Theory ................................................................................151

Implications for Theory Supported by Watson's Theory of Caring...........................151

Implications for Theory Supported by Kolcaba's Theory of Comfort .......................152

Implications for Nursing Research ...................................................................................153

Implications for Nursing Education...........................................................................156

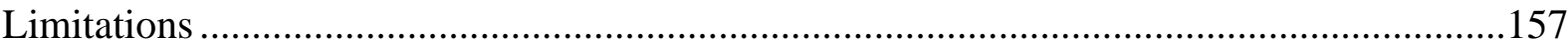


Personal Reflection on the Research Process and Results...........................................157

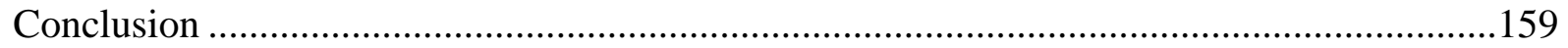

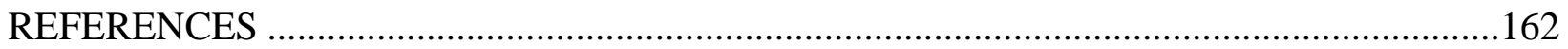

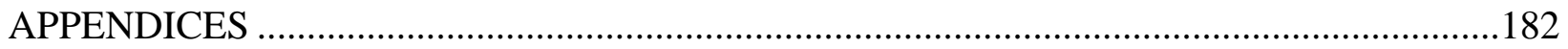

Appendix A: Empirical research on nonpharmacologic nursing interventions ...............182

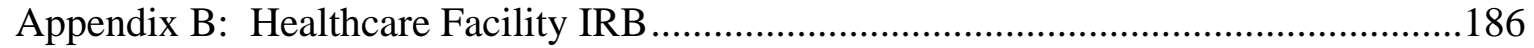

Appendix C: Healthcare Facility Research Council Approval Letter ............................187

Appendix D: ETSU Informed Consent Letter for Participants-Participant

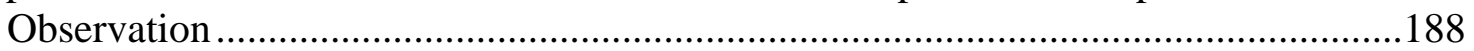

Appendix E: ETSU Informed Consent Letter for Participants— Interviews ..................190

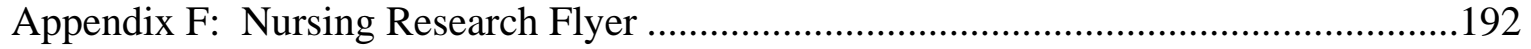

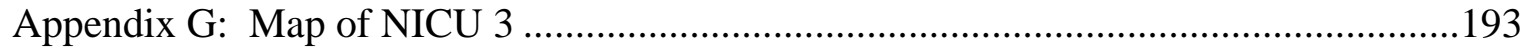

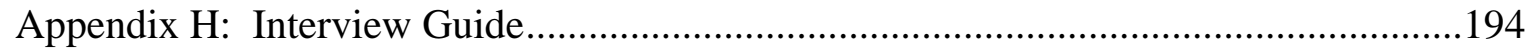

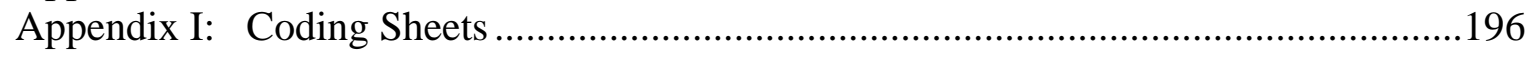

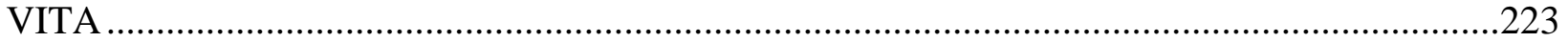




\section{LIST OF TABLES}

Table

1. Routine Care Chart 86

2. Additions to Routine Care .. 86

3. Nursing Interventions Used to Comfort Infants During Routine Care and

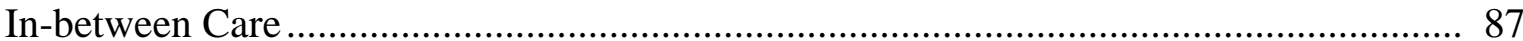

4. Field Note Observations for Environment.................................................................. 92

5. Comparisons of Performed Rest and Sleep Measures by NICU 3 Nurses .............................96

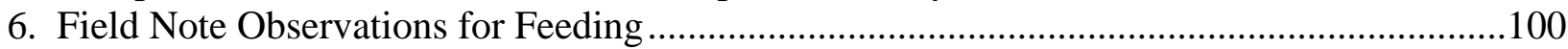

7. Field Note Observations of Interpersonal Connections of 12 Nurses................................106

8. Field Note Observations for Initial Encounter with the Baby ..........................................114

9. Recommendations for the Environment of the Infant Before Captured ...............................133

10. Recommendations for the Nurse to Provide Adequate Rest and Sleep ...............................134

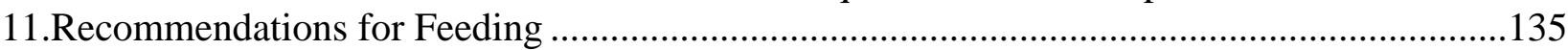

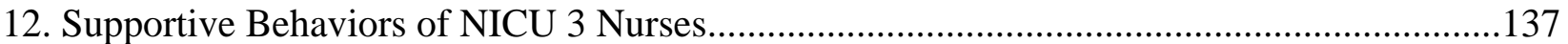

13. Recommendations for Nurturing or Comforting Behaviors From the Nurse

To the Infant 


\section{CHAPTER 1}

\section{BACKGROUND AND SIGNIFICANCE}

\section{Introduction}

Illicit drug use among pregnant women is on the rise. Current national findings indicate that illicit drug use among pregnant teenagers is $18.3 \%$. Among pregnant women aged 18 to 25 years the rate is $9.0 \%$. Women aged 26 to 44 have a 3.4\% rate of illicit drug use. (Substance Abuse and Mental Health Services Administration, 2013). Intrauterine opioid exposure can result in Neonatal Abstinence Syndrome (NAS). Newborns exposed to addicting substances during the mother's pregnancy begin withdrawal as soon as the umbilical cord is cut. Although there are many scoring tools to assess the presence and severity of NAS symptoms, there is still a lack of consensus about the relationship between neonatal outcomes and opiate use in the pregnant mother.

As the percentage of pregnant mothers who use and abuse addictive substances increases, so will the incidence of NAS. Most infants born to drug-dependent mothers will undergo NAS (between 55\% and 94\%) and will require pharmacotherapy for withdrawal symptoms (Fricker, 1978; Harper, 1974; Hudak \& Tan, 2012; Madden, 1977; Ostrea, 1976). Infants with NAS may exhibit a constellation of central nervous system, gastrointestinal, autonomic nervous system, and respiratory symptoms (Finnegan \& MacNew, 1974; Hudak \& Tan, 2012).

Because of the severity of withdrawal symptoms of NAS, many infants require pharmacotherapy to help them withdraw from maternally induced substances. Many infants also require inpatient and outpatient care during the withdrawal process. Research suggests that infant comfort measures, including a quiet environment, swaddling of the infant in a blanket, low light levels, minimal handling while sleeping, rocking, use of a swing, and generous use of a pacifier 
all contribute to decreasing the stimulation these NAS infants have difficulty handling, and contribute to the infants' wellbeing and progress (D’Apolito, 1999, D’Apolito \& Hepworth, 2001; Hudak \& Tan, 2012; Marcellus, 2002, 2007; Murphy-Oikonen, Montelpare, Bertoldo, Southon, \& Persichino, 2012). However, most nonpharmacological nursing interventions have not been empirically tested (D’Apolito, 1999; Velez \& Jansson, 2008).

Because of the increased numbers of infants with NAS being cared for in the hospital setting, Neonatal Intensive Care Unit (NICU) nurses are caring for infants with NAS along with the premature infant. Nursing interventions for infants cared for in the NICU include the assessment of vital signs, feedings, rocking, diaper changing, swaddling, placing in a swing, medication administration, use of pulse oximetry, and cardiac monitoring, but it is unknown how such interventions are tailored to the NAS infant. NICU nurses often collaborate and help each other with the care of infants. Most nurses who care for infants enjoy caring for them and providing comfort to them.

Research is also lacking in understanding the how and why NICU nurses provide care. Specifically, focused ethnography as a means to describe culture, is sparse in nursing research (Roper \& Shapira, 2000). In order to understand how and why NICU nurses do what they do, it is important to study the culture in which they work and care for infants with NAS. Culture of care is defined behaviorally (what they do), or the how, and cognitively (what they know and believe), or the why, for this study (Roper \& Shapira, 2000). Care or caring defined by Watson (1985a) is the intentional and holistic care that is provided to infants. For the purposes of this study, "culture of care" will be defined as the how and why of the intentional and holistic care that is provided to infants. Culture of care is discussed in more detail in Chapter 2. 


\section{Research Purpose, Research Questions, and Research Goals}

This purpose of this focused ethnographic study was to explore and describe the culture of care (the how and why of care) and the role of nonpharmacologic nursing interventions used by NICU nurses who provide care to infants with NAS at a hospital in the southeastern United States. One of the research goals was to identify the nurses’ meanings of their actions and beliefs by observation and individual interviews. Another goal was to bring the insider and outsider view to this ethnographic report and provide deeper insights than the nurses alone or the researcher alone (Roper \& Shapira, 2000). The last research goal was to provide general recommendations for the nurse caring for infants with NAS in regards to the nonpharmacological nursing interventions.

\section{Background and Significance}

In order to understand the nature and progression of NAS and the care of infants with NAS, a history of NAS is discussed. The most commonly employed scoring system for NAS symptoms is introduced. Next, infant withdrawal symptoms and the nursing care that is required to care for infants with NAS is also presented. Because many infants with NAS are withdrawing from maternal drugs in the inpatient setting instead of their own homes, NICU nurses are caring for these infants, who are primarily full-term, along with sick and premature infants. Exploring the NICU culture of care is crucial to understanding the nursing care provided to infants with NAS.

\section{History of NAS}

Loretta Finnegan, a pediatrician from Philadelphia General Hospital, first began to report withdrawal symptoms of newborns from drug-dependent mothers in 1969. Finnegan and MacNew's (1974) seminal article, which is quoted in almost every current article on NAS, uses 
the terms, "passively addicted infants," "Infant Withdrawal Syndrome," and "neonatal narcotic withdrawal syndrome.” Finnegan's search for previous research found one study by Perlstein (1947), which reported that narcotic addiction in mothers could cause seizures in infants during the neonatal period (Finnegan \& MacNew, 1974; Perlstein, 1974). Then, in 1975, Finnegan, Connaughton, Kron, and Emich (1975) published a landmark study in which the term "Neonatal Abstinence Syndrome” was consistently used to describe the symptoms of withdrawal in these infants. Also, in this study, the Neonatal Abstinence Scoring System (NASS) was presented as an instrument to score the withdrawal symptoms. It was in these (Finnegan \& MacNew,1974; Finnegan et al.,1975) articles that Finnegan first presented the assessment, assessment tool, and management of these withdrawing infants.

Little is published after this until the 1980s. At this point large cities were starting to see an increase in methadone use for the care of pregnant opioid-dependent women and concern for the effects of methadone on the newborn arose (Kaltenbach \& Finnegan, 1986). Currently, this concern for the effects of methadone and other addictive substances on newborns, their assessment, clinical management, and length of stay (LOS) is immense (Sarkar \& Donn, 2006). This increased incidence of NAS in infants led to further assessment and research by Finnegan.

In 1990 Finnegan and Ehrlich published the modified Finnegan scoring system called the Finnegan Neonatal Abstinence Scoring Tool (FNAST), which is in use in most NICUs today and is informally called the "Finnegan score” (Finnegan \& Ehrlich, 1990; Lall, 2008; Sarkar \& Donn, 2006). Finnegan scoring encompasses 21 withdrawal symptoms assessed observationally by nurses every 3 hours before feedings (Finnegan \& Ehrlich, 1990).

Between 2000 and 2009 a drastic increase in the incidence of NAS, maternal drug use, and hospital charges related to LOS for NAS infants has been documented in the United States 
(Patrick et al., 2012). The incidence of NAS among newborns has increased from 1.2 per 1,000 births in 2,000 to 3.39 per 1,000 births in 2009; an almost 3-fold increase (Patrick et al., 2012; Substance Abuse and Mental Health Services Administration, 2011). Uncertainty about the protocols for the reporting of NAS populations lead many to believe that the incidence of NAS has been underreported in the past (Patrick et al., 2012). Concern for the stability and coping skills of drug-using mothers in the home setting has led to increased inpatient care for infants with NAS. This drastic increase in the incidence of NAS and subsequent care of the infant with NAS in the hospital setting has led healthcare personnel to seek further standardization of treatment of NAS to decrease withdrawal symptoms and LOS (Patrick et al., 2012).

\section{Known Drugs Taken by Mothers}

According to the literature the common known drugs taken by pregnant mothers are: methadone, buprenorphine, opioids, benzodiazepines, barbiturates, alcohol, heroin, and marijuana (Finnegan \& MacNew, 1974; Hudak \& Tan, 2012; Lall, 2008). NAS is also a specific syndrome involving opioid use by the mother (Oro \& Dixon, 1987). Many mothers may use cocaine (a depressant) along with opioids; however, most research involving NAS states that recent cocaine exposure in the infant is uncommon (D’Apolito \& Hepworth, 2001). Methadone specifically, is the most used drug by pregnant mothers as they attempt to withdraw from other, more harmful drugs (Bakstad, Sarfi, Welle-Strand, \& Ravndal, 2009). Though many pregnant women who are substance abusers do not seek prenatal care, many seek addiction help by replacing their current narcotics with methadone. Methadone has a long duration of action (approximately 26 hours half-life) compared to the short half-life of heroin (Rosen \& Pippenger, 1975). In pregnancy, methadone creates a stable intrauterine environment and helps to prevent fluctuations in maternal drug levels (Hulatt, 2000). The continued use of methadone during 
pregnancy is standard pharmacotherapy for opioid-dependent women in order to prevent the miscarriage of the infant. When methadone is combined with prenatal care, there are improved obstetric and neonatal outcomes. (Gray et al., 2010).

A lesser-used alternative for opioid dependence is buprenorphine, which was approved by the U.S. Food and Drug Administration (FDA) in 2002 (Rayburn \& Bogenschutz, 2004). The pregnant woman's use of methadone maintenance therapy (MMT) and buprenorphine maintenance therapy (BMT) both prolong the LOS for NAS infants. The dose-response connection between maternal methadone and buprenorphine levels and infant withdrawal has been studied but remains unclear and inconsistent (Pritham, Paul, \& Hayes, 2012). Therefore, there are many factors that can affect the symptoms and the severity of withdrawal in these fragile infants (Johnson, Greenough, \& Gerada, 2003; Lim, Prasad, Samuels, Gardner, \& Cordero, 2009; Pritham, Troese, \& Stetson, 2007; Pritham et al., 2012; Seligman et al., 2008). Lastly, many women are poly-drug users (narcotics and methadone or buprenorphine), and physicians and nurses are unaware of this until the delivery of the infant. This poly-drug use in the mother brings many challenges to the care of drug-dependent newborns.

\section{Infant Withdrawal Symptoms}

The literature suggests that withdrawal symptoms begin anywhere from 8 to 72 hours after birth with a possible delayed onset of 4 weeks (Finnegan \& MacNew, 1974; Kandall \& Gartner, 1974; Lim et al., 2009). Cord clamping is the initiating event, which begins withdrawal for the infant (Nelson, 2013). As the circulating levels of the mother's addictive substances decrease in the infant, the infant begins to show signs and symptoms of NAS. Therefore, the newborn nursery nurse may be the first to observe withdrawal symptoms in these infants. The mother's lack of prenatal care, very late prenatal care (after 24 weeks), abruptio placentae, 
preterm labor with no obvious cause, or intrauterine growth retardation with no obvious cause will alert healthcare personnel of possible drug addiction in the mother and subsequent drug dependence and withdrawal in the newborn. However, many infants and mothers may be discharged to the home environment before the observance of withdrawal symptoms, which poses further health problems for the infant such as excessive crying, feeding problems, respiratory issues, and seizures.

Beginning with Finnegan (1974), to the current American Academy of Pediatrics (AAP) statement on NAS (2012), there is a general agreement on the specific cluster of symptoms that encompass the newborn with NAS. The withdrawal symptoms are exhibited through the central nervous system (tremors, irritability, high-pitched cry, abnormal suck, excessive sucking or poor feeding, seizures), autonomic nervous system (sneezing, yawning, mottling), gastrointestinal (diarrhea, loose or watery stools, vomiting), and pulmonary (increased apnea and respiratory distress) and can be evidenced at varying degrees (Finnegan, 1974; Hudak \& Tan, 2012). Although normal newborn care can be challenging for nurses, the care of the extremely complex infants with NAS can be stressful and frustrating due to their fluctuating and extreme withdrawal symptoms (Murphy-Oikonen, Brownlee, Montelpare, \& Gerlach, 2010).

\section{Nursing Care of the NAS Infant}

Nurses provide the assessment, medication administration, and care of the drugwithdrawing infant. After the infant is assessed for withdrawal symptoms in the newborn nursery and is diagnosed with NAS, these infants are then transferred to a NICU for specialized withdrawal care. Many times, the mothers of these infants are not present to provide care to their infants. The nurse assesses, feeds, and comforts the infant during withdrawal. These infants need to be rocked, have a quiet and calm atmosphere (darkened room, alarms turned down, and 
quieter voices), have a pacifier provided, and have human contact. Adequate nutritional intake is required to meet their increased caloric needs (D’Apolito, 1979; Hudak \& Tan, 2012; MurphyOikonen et al., 2012).

If the Finnegan score is used to assess withdrawal symptoms, the infant will be observed before feedings and a score will be given. This 21-item assessment of infant withdrawal symptoms is one of two published abstinence-scoring tools recommended by the AAP (Hudak \& Tan, 2012). Another scoring system, the Lipsitz tool (also known as the Neonatal Drug Withdrawal Scoring System), scores symptoms from zero to 20 (tremors, irritability, hyperreflexia, increased muscle tone, and tachypnea) (Lipsitz, 1975). Both the Finnegan and the Lipsitz tools have been recommended by the American Academy of Pediatrics (AAP, 1998). Currently, Finnegan scoring tool is the most widely used tool in the United States and Canada (Marcellus, 2002). If an infant obtains two consecutive Finnegan scores $>8$, pharmacotherapy is usually initiated. NICUs are encouraged to implement a weaning protocol to help the infant's withdrawal from maternal substances (Hudak \& Tan, 2012). Morphine is the most common drug administered to infants to help them withdraw from maternal substances. Phenobarbital and clonidine are adjunct medications that are also used for drug withdrawal in these infants (Finnegan \& MacNew, 1974; Lim, Prasad, Samuels, Gardner, \& Cordero, 2009). However, Sarkar and Donn's (2006) national survey on the management of NAS reports that only 55\% of surveyed NICUs use and implement a written protocol for withdrawal from NAS. The uses of written clinical practice guidelines (CPG) by healthcare personnel are recommended for the care of infants with NAS in order to decrease withdrawal symptoms and, possibly, length of stay (Hudak \& Tan, 2012; Murphy-Oikonen et al., 2012). 


\section{Length of Stay}

According to the severity of the withdrawal, the duration of symptoms is anywhere from 6 days to 8 weeks (Finnegan \& MacNew, 1974; Patrick et al., 2012). Studies of inpatient populations of infants with NAS report a wide range of length of stay (LOS) ranging from 1 to 122 days (Agthe, Kim, \& Mathias, 2009; Burns \& Mattick, 2007; Johnson et al., 2003; Seligman et al., 2008). The implementation of clinical practice guidelines (CPG) for inpatient care has proven to decrease withdrawal symptoms in infants (Murphy-Oikonen et al., 2012). The Murphy-Oikonen et al. (2012) study distinguishes between infants treated in the newborn nursery and those treated in the NICU. NICU care has been shown to decrease LOS compared to newborn nursery care for methadone-exposed infants (Murphy-Oikonen et al., 2012). Inpatient pharmacologic weaning protocols, the excessive withdrawal symptoms, training in Finnegan scoring for NAS symptoms, prolonged care, and lack of parental involvement require more specialized nursing and medical care for these newborns (Hudak \& Tan, 2012; Hulatt, 2000; Patrick et al., 2012). Rooming in with the mother has been associated with decreased withdrawal symptoms and shorter LOS. However, in these experiences, routine care for the mother include teaching on-infant care, breastfeeding instructions (if needed), and encouragement by the nursing and medical staff. Although this method of rooming in with the mother and infant proved effective, many drug-abusing mothers would not consent to this, and many facilities could not practically allow for the cost of caring for both the mother and the infant (Abrahams et al., 2007). Although a combination of inpatient and outpatient treatment for NAS has been studied with evidence for decreased LOS, most healthcare providers have not adopted this approach to care (Backes et al., 2012). The lack of identification in the literature of the location for inpatient care of NAS infants (newborn nursery, NICU, rooming with the mother, or combination inpatient or 
outpatient care) makes LOS data difficult to interpret. Although each infant metabolizes and responds differently to the drugs obtained from the mother before and at birth, LOS and the factors that decrease LOS continue to be issues that need to be addressed for the best outcomes for these infants and for the NICUs where they receive care (Hudak \& Tan, 2012).

\section{NICU Care of the NAS Infant}

Most of the infants cared for in the NICU are premature or sick-term infants who require the specialized care of a neonatologist and NICU trained nurses. NICU trained nurses usually do not have advanced or special training to care for the infant with NAS (Sarkar \& Donn, 2006). Neonatal nurses and health care providers are encouraged to create an environment that closely resembles the mother's womb. Environmental factors (light, noise, and touch) in the NICU can cause stress on the preterm and term infant and discourage proper growth and development (Long, Philip, \& Lucey, 1980; Mann, Haddow, Stokes, Goodley, \& Rutter, 1986). Infants cared for in the NICU live in an artificial environment that is unlike the in utero environment; thus unnatural light, increased noise, and repeated disturbances (gentle touch and painful touch) can all deliver stress to the preterm or sick infant (Als et al., 2004). The reduction of light to correlate with circadian rhythms and the reduction of noise to prevent overstimulation have long been the interventions to help the infant rest and sleep and to mimic the mother's womb (Levy, Woolston, \& Browne, 2003; Mann et al., 1986; Miller, White, Whitman, O’Callaghan, \& Maxwell, 1995).

Neonatal stress was considered an important topic in research in the early 1980s. Als, Lester, Tronick, and Brazelton (1982) developed the Newborn Individualized Developmental Care and Assessment Program (NIDCAP) to provide a combination of strategies to decrease stress on premature infants. Control of environmental stimuli (auditory, visual, and tactile) and positioning of the infant were all hypothesized to provide holistic care to improve developmental 
outcomes (Als et al., 1986). As vulnerable infants responded to light, noise, and handling, it was concluded that these areas of over stimulation could inhibit normal growth and development (Als et al., 1986, Als et al., 2004). However, current research on the NIDCAP with preterm infants reports positive effects and no effects on preterm infants (Als et al., 2004; Jacobs, Sokol, \& Ohlsson, 2002; Maguire et al., 2009). To date no research exists that uses the NIDCAP to assess neonatal stress on infants with NAS.

Another concept in the NICU is "developmental care," a term used to describe the individualized care for the preterm infant that includes decreased stimulation of light, noise, and touch (Aita \& Snider, 2002; Als, 1982; Reiser, 1996). Terms in the literature that encompass decreased stimulation are "decreased touch,” "decreased light," "decreased noise,” “coordination of care,” and "cluster care” (Peters, 1999). Currently, most NICUs follow protocols to decrease stimulation for preterm and sick infants (Hack, 2009; White, 2011). Although some infants with NAS are initially cared for in newborn nurseries, most inpatient care for infants with NAS is in the context of the NICU. Therefore, many of the aspects of developmental care involving decreased stimuli to the infant with NAS originate from the care of preterm or sick infants.

\section{Significance of the Study}

NAS is an increasing healthcare and social problem that is currently being researched through the quantitative lens and through the medical model (Hudak \& Tan, 2012; Patrick et al., 2012). Few studies exist to describe why and how infants with NAS are cared for. Even more narrow is the lack of qualitative studies exploring the culture of care for infants with NAS withdrawing in the inpatient hospital setting. Therefore, this study is my effort to holistically 
describe the culture of care provided by NICU nurses to infants with NAS to potentially help nurses and provide knowledge that is useful for clinical practice (Roper \& Shapira, 2000)

\section{Philosophical Underpinnings}

The philosophical basis of this study is that of constructionism. Interpretive paradigms that seek understanding of the world are socially constructed (Denzin \& Lincoln, 2000). These social expressions of culture allow for reconstructed understandings of the social world; therefore, complex views are uncovered instead of a narrowing of views to a few categories or ideas. Reality is “constructed” by social interaction. This philosophy does not start with a theory, but concepts are inductively developed into patterns of meaning (Denzin and Lincoln, 2000). Therefore, constructionism is in agreement with the method of focused ethnography.

\section{Summary}

A culture of care requires leadership, detailed language in how to provide a culture of care, and a specific community to care for. NICU nurses also have developed a culture of care for infants with NAS by using many of the nursing interventions used for premature and sick infants and by the "passing down" of a culture of caring for infants from nurse to nurse. A literature review of the five nonpharmacological nursing interventions follows as well as examples of culture in healthcare settings. Nursing interventions are part of a culture of care that nurses provide to patients. The nonpharmacological interventions are learned from other nurses in orientation, training, and on-going educational opportunities. These interventions are also learned in the how and why of providing care to infants in a culture sharing experience. 


\section{CHAPTER 2}

\section{LITERATURE REVIEW}

\section{Introduction}

NICU nurses perform many nursing interventions to care for the fragile infant with NAS. Little research has been conducted for the nursing interventions that are provided to infants with NAS. The literature search resulted in five main areas of nonpharmacological nursing interventions performed by nurses for infants with NAS: environment, adequate rest and sleep, feeding, Finnegan scoring, and nurses as caregivers. Each of these nonpharmacological interventions has been reviewed in the literature with detailed findings for each intervention. Appendix A provides a condensed view of the findings. This chapter also includes recommendations for future research. In order to describe a culture of care for infants with NAS, the how and why of culture need to be explored. Some of the how and why are in the form of nursing interventions.

A systematic and thorough review of the literature was conducted on the nursing care of infants with Neonatal Abstinence Syndrome. A CINAHL, Nursing and Allied Health, PubMed, and Cochrane Database of Systematic Review database search was conducted with the keywords neonatal abstinence syndrome, nursing, nursing care, environment, light, noise, touch, swaddling, non-nutritive sucking, rocking, positioning, infant feeding, formula, breastfeeding, breast milk, Finnegan score, surrogate mothers, nurses as mothers, length of stay, culture, caring, caring, culture of care, in-patient, and out-patient. Each term was searched independently in combination with the term neonatal abstinence syndrome. Abstracts and reference lists were reviewed as well. Most of the literature surrounding NAS involves pharmacotherapy and synthesis of literature reviews for infant care. The 57 research studies on 
NAS in the Cochrane Database of Systematic Reviews yielded only two that studied nursing interventions: water-bed care and rocking bed vs. standard beds (D’Apolito, 1979; Oro \& Dixon, 1988). The Proquest Dissertation and Theses database yielded one result on breastfeeding (Gerlach, 2010). Most of the research involving NAS originated years ago and is not considered current except for the research on feeding, Finnegan scoring, and nurses as surrogate mothers. Initially, 277 articles were found using the keywords. The titles and abstracts were then reviewed for relevance and quality. The final sample included 16 reports in the English language.

Whittemore's (2005) integrative review criteria were used to analyze the literature for quality. Each research study was scored on a scale of 1-11 and categorized as "excellent" (score of 9 or greater) or "good" (score of 6 or greater but less than 9). Studies that were not considered “excellent” or "good" were excluded. The inclusion criteria for selection were articles that (a) addressed methodological rigor; (b) used quantitative or qualitative research strategies; and (c) involved nursing care/interventions for NAS infants.

The primary disciplines reviewed were nursing, medicine, social work, psychology, and addiction. NAS is an international social phenomenon; therefore, all articles written outside of the United States and published in the English language were included. Research studies on the nursing care of infants with NAS encompassed six main areas of practice: environment (light, noise, and touch), adequate rest and sleep (use of swaddling, nonnutritive sucking, rocking, and positioning), feeding (formula and breastfeeding), Finnegan scoring by nurses, nurses as caregivers, and pharmacotherapy. Although medication administration is considered a nursing intervention, the prescribing of medications for infants with NAS falls outside the realm of independent nursing interventions; therefore, pharmacotherapy research studies were excluded from this analysis. Nursing literature is abundant with literature reviews about specific clinical 
issues of NAS and the care of these infants; however, actual empirical research on nursing interventions is scarce.

\section{Gap Between Recommendations and Knowledge}

In 2012 the AAP released a policy statement on neonatal drug withdrawal challenging clinicians to "include evidence-based approaches to the management of the hospitalized infant who requires weaning from analgesics or sedatives” (Hudak \& Tan, 2012, p. e540). The expert recommendations from the Committee on Drugs and the Committee on Fetus and Newborns include protocols for screening of maternal and infant substances, pharmacotherapy, assessment of withdrawal symptoms using a published assessment tool, and nonpharmacologic supportive measures. The nonpharmacologic supportive measures include "minimizing environmental stimuli, promoting adequate rest and sleep, and providing sufficient caloric intake to establish weight gain” (Hudak \& Tan, 2012, p. e540).

The question arises, then, as to what is the culture of care provided by NICU nurses for infants with NAS as it relates to nonpharmacologic nursing interventions? Velez and Jansson (2008) reported that there is little clinical research to support nursing interventions to help decrease withdrawal symptoms. Most of the published literature of listed nonpharmacologic interventions for NAS is based on previous research on premature or sick infants cared for in the NICU. Because most NAS infants are cared for in the NICU by NICU nurses, these same nonpharmacologic interventions (decreased stimuli with light and noise, adequate rest and sleep, and the provision of sufficient caloric intake to establish weight gain) have been applied to the NAS population. Because newborn nursery nurses and NICU nurses provide care for infants with NAS, evidence-based nursing interventions are needed for the nurse to help decrease withdrawal symptoms, provide surrogate parental care of the newborns, and decrease LOS. 


\section{Nonpharmacological Nursing Interventions}

\section{Environment (Light, Noise, Touch)}

Empirical literature regarding the intervention of decreasing environmental stimuli for infants with NAS is limited. Ostrea, Chavez, and Strauss (1976) reported the first study of the effect of the nursery environment on infants with NAS. Findings revealed that decreasing the amount of light and noise in the nursery did not lower the incidence of withdrawal symptoms in drug dependent infants. However, in their earlier observational study of withdrawal behaviors using the Brazelton Neonatal Behavioral Assessment Scale (NBAS), assessment revealed that addicted infants were less capable of maintaining an alert state and became more irritable with the stimuli of light and noise, especially at 48 hours of age (Strauss, Lessen-Firestone, Starr, \& Ostrea, 1975). Regardless of the contradiction, the Ostrea et al. (1976) nursery intervention research confirmed that signs of withdrawal in the drug dependent infant are internal (irritability, tachypnea, tremors, diarrhea, vomiting) as well as external (light, noise, touch). Observed internal factors match 5 of the 21 withdrawal symptoms reported by Finnegan and MacNew (1975) at this time, which reflect the infant's withdrawal from maternal drugs. Many authors suggest interventions of dim lighting, quiet rooms, and infant handling in a slow and gentle manner (Finnegan \& MacNew, 1974; Jansson \& Velez, 1999; Murphy-Oikonen et al., 2012; Velez \& Jansson, 2008); however, these interventions have not been systematically tested for infants with NAS since the Ostrea et al. (1976) study.

\section{Adequate Rest and Sleep (Swaddling, Nonnutritive Sucking, Rocking, Positioning) \\ O’Brien and Jeffrey (2002) found that sleep deprivation, disorganization (indeterminate sleep), and fragmentation (arousals to wakefulness) were found in newborns with NAS at the onset of treatment. Sleep disturbances were found to be associated with the severity of}


withdrawal from maternal drugs. Greatest differences in sleep disturbances occurred in infants with NAS receiving treatment (oral morphine) over infants with NAS not receiving treatment (O’Brien \& Jeffrey, 2002). O’Brien and Jeffrey (2002) hypothesized that sleep disturbances for infants receiving oral morphine could be a result of CNS changes throughout opiate dependency in utero and CNS hyperactivity during opiate withdrawal after birth, although the mechanisms remain unclear. Similar findings for sleep disturbances in infants receiving oral morphine were found by Anders, Emde, and Parmalee (1971), Dinges, Davis, and Glass (1980), and Pinto, Torrioli, Tempesta, and Fundaro (1988). The O’Brien and Jeffrey (2002) observational research was the first to suggest that sleep changes may be due to opiate dependence in utero. To date there are no researched intervention studies to promote adequate sleep for the infant with NAS.

Supine sleep for the prevention of Sudden Infant Death Syndrome (SIDS) is part of standard newborn care and teaching (AAP, 2011). To date there is no research on supine sleep with infants with NAS and the incidence of SIDS.

Swaddling. Nurses routinely swaddle infants to mimic the comfort of the womb. This intervention also decreases the Moro reflex, which can wake or aggravate an infant. Swaddling is a well-known practice to prolong sleep in healthy infants (Franco et al., 2004; Gerard, Harris, \& Thach, 2002). Infant swaddling is also an ancient practice that has recently gained popularity through a book and promotional program by Harvey Karp (2002). Though there are many swaddling techniques, it has been proven that some forms of tight leg swaddling have been associated with developmental dysplasia of the hip (DDH) (Kutlu, Memik, Mutlu, Kutlu, \& Arslan, 1992). Tight chest swaddling is linked to increased risk for pneumonia and is discouraged in infant care (Yurdakok, 1990). However, contemporary swaddling techniques with infants snugly wrapped with their hips safely flexed and abducted - have shown beneficial 
results. Swaddling implemented correctly is shown to reduce crying in infants (Van Sleuwen et al., 2007), improve sleep (Franco et al., 2004; Gerard et al., 2002; Richardson, Walker, \& Horne, 2010), and shorten periods of distress (Franco et al., 2004; Ohgi, Akiyama, Arisawa, \&

Shigemori, 2004). Although nurses swaddle healthy, premature, and drug-exposed infants, there is currently no empirical evidence for the intervention of swaddling to decrease the number or amount of withdrawal symptoms in infants with NAS.

Nonnutritive sucking. Nonnutritive sucking has been established as an effective method for the relief of pain in newborns and preterm infants, especially during painful procedures (Liu, Lin, Chou, \& Lee, 2010). Liaw et al. (2013) reported a study in which more quiet-sleep occurred during heal sticks with a combination of nonnutritive sucking, sucrose, and facilitated tucking (swaddling) of the infant than those not receiving these interventions. Although most of the published literature suggests the generous use of a pacifier to calm the infant and decrease withdrawal symptoms, no empirical research exists for the use of nonnutritive sucking for infants with NAS.

Rocking. Mothers have often used rocking to calm irritable infants and to lull them to a quieter state (Barnard, 1972). Rocking to decrease withdrawal symptoms in infants with NAS was the impetus for D’Apolito’s (1999) rocking bed versus standard bed intervention research. D’Apolito (1999) found that when infants' withdrawal symptoms were recorded using mechanical rocking beds versus standard beds, the rocking bed use did not decrease withdrawal symptoms and actually resulted in overstimulation of the infants. D’Apolito and Hepworth (2001) in a secondary analysis of data from the previous rocking bed study (D’Apolito, 1999), found that polydrug-exposed infants demonstrated increased muscle tone (41\%), disturbed sleep (77\%), and crying (23\%) from day 3 to day 7 with the use of rocking beds. Although the nursing 
interventions of swaddling (to control tremors, decrease the Moro reflex, and facilitate sleep) and offering a pacifier (for nonnutritive sucking) were suggested, this was not an interventional study (D’Apolito \& Hepworth, 2001).

Waterbeds have been used for premature infants to reduce apnea, increase sleep periods, decrease crying and irritability, and improve growth (Korner, Kraemer, \& Haffner, 1975; Korner, Schneider, \& Forrest, 1983). Oro and Dixon (1988) used nonoscillating waterbeds and conventional bassinets for infants with NAS. Decreased CNS symptoms permitted the infants on waterbeds to require less pharmacology to decrease symptoms and assisted with earlier weight gain (Oro \& Dixon, 1988). D’Apolito’s (1999) and Oro and Dixon’s (1998) intervention studies were both attempts to decrease CNS symptoms of withdrawal in order to promote adequate rest and sleep. However, the fact that rocking beds $(n=14)$ provided overstimulation, which increased withdrawal symptoms of the infant and nonoscillating waterbeds $(n=30)$ decreased withdrawal symptoms, suggest that more research is needed for rocking beds and waterbeds with possibly larger sample sizes.

Positioning. Maichuk, Zahorodny, and Marshall’s (1999) research study on positioning of infants with NAS found that swaddled prone lying infants $(n=25)$ proved to have decreased withdrawal symptoms over swaddled supine lying infants $(\mathrm{n}=23)$. Although prone lying infants experience less crying and have decreased energy requirements, current standards to prevent Sudden Infant Death Syndrome (SIDS) require that all infants sleep supine for safe sleep (AAP, 2011; Maichuk et al., 1999).

\section{Feeding (Formula and Breastfeeding)}

The breastfeeding of infants has become the gold standard for infant nutrition (Lawrence, 2010). Breastfeeding is now supported by research for its long-term medical and 
neurodevelopmental advantages over infant formula (International Lactation Consultant Association, 2004). The AAP recommends exclusive breastfeeding for 6 months along with continued breastfeeding for 1 year or longer with age-appropriate foods (AAP, 2005; AAP, 2012b). Exclusive breastfeeding is also supported by the World Health Organization (2012), the Centers for Disease Control (2013), and International Lactation Consultant Association (2004). However, mothers maintained on methadone usually have low rates of breastfeeding due to a lack of coping skills and physical, sexual, and emotional abuse (Velez et al., 2001). Infants with NAS may experience intrauterine growth restriction and/or premature birth (Bolnick \& Rayburn, 2003). Along with their possible low birth weight, infants with NAS undergo withdrawal symptoms that expend energy and deprive sleep (Wilson, 1975); therefore, caloric needs are increased. Hill and Desmond (1963) and Wilson (1975) report that most infants with NAS require double the caloric needs of a normal newborn due to vomiting, loose stools, and increased energy expenditure. Nurses provide feedings to infants with NAS in the form of formula and expressed breast milk and helping the infant's mother breastfeed, which requires teaching and skill for the nurse. Infant feeding goals are to facilitate sleep to conserve energy and to have adequate nutritional intake to establish consistent weight gain (Hudak \& Tan, 2012).

Breastfeeding and the use of expressed breast milk are recommended and are related to less severe withdrawal symptoms (Abdel-Latif et al., 2006; Isemann, Meinzen-Derr, \& Akinbi, 2011). No studies exist that test the effectiveness of formula feedings alone or type of formula fed to infants with NAS on weight gain. Also, no studies exist for the use of banked human milk for infants with NAS. However, LOS has been reported shorter in breastfed infants over formulafed infants or in infants fed a combination of formula and breast milk (Pritham et al., 2012).

Quality research studies on the effects of methadone levels in breast milk are few. Eight 
research studies comparing the use of breast milk and formula as a feeding method for infants with NAS were evaluated. Six of the eight studies used a published abstinence-scoring tool to assess withdrawal symptoms. One of the eight studies used LOS data and another used nine neurobehavioral outcomes to assess the infants. Ballard (2001) found that breast fed infants exhibited decreased withdrawal symptoms and decreased LOS; however, with a small sample size ( $n=6$ ), the results were limited. Abdel-Latif et al. (2006) were the first to use an adequate sample size (breast milk: $\mathrm{n}=85$; formula: $\mathrm{n}=105$ ) and found that Finnegan scores were significantly lower in the breast milk group over the formula fed group (after stratifying for prematurity and polydrug and methadone exposure). Significantly fewer infants required weaning with oral morphine and the median time for withdrawal occurred later in the breast fed group (Abdel-Latif et al., 2006). Jansson et al. (2008) found that fewer infants required pharmacology for NAS although these findings were not statistically significant (formula: $n=4$; breast: $\mathrm{n}=1$ ). All infants were assessed for nine neurobehavioral symptoms (attention, quality of movement, regulation, nonoptimal reflexes, stress or abstinence, arousal, excitability, and lethargy) on days 3, 14, and 30 with no significant affects. Jansson et al. (2008) found that these results contribute to previous recommendations for breastfeeding for mothers on MMT if it is appropriate. Gerlach (2010), in a retrospective cohort study in Canada measured methadone exposed infants (breast: $\mathrm{n}=8$; formula: $\mathrm{n}=9$, combination: $\mathrm{n}=11$ ) and found that the infants who were breastfed exhibited less overall neonatal abstinence symptoms when looking at the magnitude, duration, and area of scores. Finnegan scores for withdrawal symptoms were low compared to infants who were formula fed or fed a combination of breast milk and formula in this epidemiological study (Gerlach, 2010). Gerlach recommended that larger sample sizes be used for further research. Dryden, Young, Hepburn, and Mactier (2009) used the Lipsitz scoring 
tool to assess 450 infants with NAS. Findings revealed that after breastfeeding for 72 hours, only half of the infants required pharmacotherapy (Dryden et al., 2009). This large sample size adds to the reliability of the findings. McQueen, Murphy-Oikonen, Gerlach, and Montelpare (2011) found predominantly breastfed infants have significantly lower Finnegan scores; suggesting decreased severity and duration of withdrawal symptoms when compared with infants who were fed a combination of breast milk and formula or formula alone (breast milk: $\mathrm{n}=8$; combination: $\mathrm{n}=11$; formula: $\mathrm{n}=9$ ). Research revealed that breastfeeding may offer withdrawal benefits to methadone exposed NAS infants (McQueen et al., 2011). Isemann et al. (2011) found that breastfeeding is associated with shorter median duration of pharmacological treatments in preterm and term infants. Breastfeeding infants also had decreased LOS when compared to formula fed infants (breast: $\mathrm{n}=56$; formula: $\mathrm{n}=56$ )(McQueen et al., 2011). Newest research on breastfeeding by Pritham et al. (2012) revealed LOS was shorter in breastfed infants than formula-fed infants or infants who received formula and breast milk. Neonates with prenatal exposure to methadone $(\mathrm{n}=136)$ spent more days in the hospital (21 versus 14 days) for treatment of NAS than those prenatally exposed to buprenorphine $(n=16)$. These findings are consistent with previous studies for decreased LOS with the breastfeeding group. Pritham et al. recommend a larger sample size for the buprenorphine group for future research. The findings from each of these studies support the AAP (2012b) recommendation for mothers who are on MMT to continue breastfeeding. However, each mother needs to be evaluated individually for poly-drug use, coping skills, and contraindications of HIV status (Jansson, Velez, \& Harrow, 2004).

\section{Finnegan Scoring}

Systematic use of abstinence assessment tools is recommended for infants with NAS to 
provide adequate interventions (Hudak \& Tan, 2012). Finnegan et al. (1975) developed the first scoring system for NAS called the Neonatal Abstinence Scoring System (NASS). This primary work describing this scoring system is cited in most articles on NAS. In 1990 Finnegan published the modified Finnegan Neonatal Abstinence Scoring Tool (FNAST), which is in use in most NICUs today and is informally called the "Finnegan Score.” The Finnegan score is comprised of 21 withdrawal symptoms that are scored every 3 hours before feedings. This multidimensional scoring of the infant produces no burden on the infant because the Finnegan score is an observational assessment. Withdrawal symptoms are divided into central nervous system symptoms, metabolic or respiratory symptoms, and gastrointestinal symptoms. Infants receiving a score $>8$ on two consecutive scores will usually receive morphine to help to ease withdrawal symptoms. Phenobarbital and clonidine are adjunct medications that are also used for the drug withdrawal in these infants. The Finnegan score is also the only scoring tool that provides consistency for pharmacotherapy use. Techniques learned by using the Finnegan scoring tool are appropriate for nurses to give consistent scores for effective treatment of the infant with NAS (D’Apolito \& Finnegan, 2010; Finnegan et al., 1975).

The applicability to practice of the Finnegan scoring tool resides in the fact that the actual withdrawal symptoms of NAS are the symptoms assessed on the Finnegan Score. Finnegan Scoring provides unique and specific data for the infants' withdrawal symptoms and their subsequent management. Many NICU nurses receive training and education in observation of signs and symptoms and scoring. D’Apolito (1999) reported that content validity of the Finnegan tool has been clinically tested with more than 200 infants and staff inter-observer reliability scores were 90\% or greater. However, Murphy-Oikonen’s (2012) research reported that reliability and validity data for the Finnegan scoring tool are lacking; however, many studies 
reported the tool to be extensively used to assess and guide treatments for NAS. Continued research for the validity and inter-rater reliability of the Finnegan scoring tool is needed (Hulatt, 2000; Murphy-Oikonen et al., 2010). Nurse's scoring of infants can be subjective, therefore; adequate training and education is needed to prevent inaccurate scores, inappropriate treatment, and increased LOS (Lucas \& Knobel, 2012).

Sarkar and Donn's (2006) national survey data on the management of NAS infants in the NICU setting report that only $70 \%$ of respondents use an abstinence scoring system to determine when to initiate or stop pharmacologic treatment. Inconsistency in clinical practice can prohibit evidence-based practice for the care of infants with NAS (Sarkar \& Donn, 2006). Current quality research on the nurse's education and consistent use of the Finnegan scoring tool is sparse. Lucas and Knobel (2012) implemented practice guidelines and education for the Finnegan scoring tool with NICU nurses to answer the research questions: Will delivering an educational program to nurses in the NICU around NAS and the use of the FNAST result in increased knowledge as evidenced by a higher score on the NAS test, and will the educational program result in increased accuracy in using the FNAST to score an infant with NAS? Expert content validity of the knowledge was obtained from Finnegan on the pretest and posttest. Although the sample size was small $(n=10)$, study participants scored $90 \%$ or greater on the posttest 1 week after completion of the educational program. Inter-rater reliability program guidelines require an achievement of $90 \%$ or greater on consistency of scores (D'Apolito \& Finnegan, 2010). Many NICU nurses may participate in D’Apolito and Finnegan's (2010) Inter-Observer Reliability Program (DVD); however, Lucas and Knobel's (2012) research is the only published report on this educational program. Evidence-based clinical guidelines and education of NAS and Finnegan scoring provide healthcare personnel with the necessary tools to accurately assess the 
drug-exposed infant (Lucas \& Knobel, 2012).

The greatest advantage of the Finnegan Scoring system is that it was developed specifically for infants with NAS. The 21 withdrawal symptoms assessed are specific to infant withdrawal from maternally induced drugs. The benefit of this tool is that it provides an objective means to validate treatment. The disadvantage is that the scoring may be subjective if assessors are not adequately trained using evidence-based methods of education. Although most research studies use the Finnegan Score as a means of reporting NAS symptoms and treatment, there has been little empirical research of nursing education to test the inter-rater observability of nurses using the Finnegan score (Murphy-Oikonen et al., 2012).

\section{Nurses as Caregivers}

Due to the social and personal consequences of addiction in the mother, many times the nurse is the caregiver or "mother” for the hospitalized infant with NAS (Velez \& Jansson, 2008). Research supports that the early environment and influence of caregivers and attachment in the first few years of life impact early right brain development. Efficient right brain function (through attachment of the infant to the primary caregiver) provides future adaptive coping skills for future relational development (Schore, 2001). When the mother is absent from the NICU or only visits occasionally, the nurse takes over the caregiving responsibilities of maternal care of the infant with NAS (Murphy-Oikonen, Brownlee, Montelpare, \& Gerlach, 2010). Withdrawal symptoms of infants with NAS (irritability, excessive crying, and lack of sleep) can be difficult for nurses. This demanding role can cause frustration and burnout (Murphy-Oikonen, et al., 2010). Most of the NAS research provides nurses with suggestions for care of the drugdependent newborn (swaddling, rocking, use of a pacifier, feeding, and consoling) with nurses and parents delivering this “mothering” care to the infant (Velez \& Jansson, 2008; Murphy- 
Oikonen et al., 2012). With many drug-addicted mothers absent from the NICU, nurses provide and supplement this care.

Research on nurses as caregivers for infants with NAS is limited. Fraser, Barnes, Biggs, and Kain (2007) reported in their interpretive methods study that the nurse's relationship to the infant with NAS is time consuming and difficult. Many nurses felt that their care of the infant had to be balanced between treatments for the infant with NAS and time spent consoling the infant. Group interviews with nurses revealed that the workload was very demanding. Tensions in the work environment were also discussed in relation to the location of NAS infant care. Questions rose as to the optimal environment for NAS infant care: the NICU setting, or separate rooms for the infant and mother to promote attachment. This study concluded with nurses wanting to make a difference in the lives of these infants and mothers. Empathy for infants with NAS and their mothers was a predominant characteristic of the NICU nurse. However, nurses agreed that more education is needed to care for these infants and mothers in a comprehensive manner that facilitates parental commitment and caregiving skills (Fraser et al., 2007).

Murphy-Oikonen et al.’s (2010) qualitative study on the experiences of NICU nurses caring for infants with NAS revealed that nurses experience numerous expectations to provide maternal care to infants. The NICU nurse is highly trained in technical skills; however, there was a conflict between the provision of these skills and at the same time the responsibility of consoling demanding infants. Regardless of the demands of NICU nurses caring for infants with NAS, these nurses continue to possess love and commitment to newborns (Murphy-Oikonen et al., 2010). Concern for the infant's welfare after the infant is discharged from the hospital reflects the caring feelings of NICU nurses. The theme of commitment to newborns was revealed in this study (Murphy-Oikonen et al., 2010). Despite the difficulty in caring for these infants, 
both Fraser et al. (2007) and Murphy-Oikonen et al. (2010) portrayed the NICU nurse as one who provides "maternal" care for the infants, especially in the absence of the mother.

\section{Guiding Theories}

Nursing theory guides nursing practice by providing an efficient means of collecting data in order to predict nursing practice (Alligood \& Mariner-Tomey, 2010). The use of nursing theory in nursing research clarifies the purpose of nursing practice and differentiates it from other caring professions (McEwen \& Wills, 2011). With the drastic increase in numbers of infants with NAS (reported and not reported) and research on best practices to care for these fragile infants, nursing theory is needed to provide the framework and narrative for models of care.

\section{Culture of Care}

Nurses, as they care for patients, operate in a culture that provides care to specific patients with specific needs. Each nursing unit has an individual culture of care. The term "culture of care" can be defined in numerous ways; however, literature describing this term is limited. In order to provide a clear description and definition of culture of care for this research, the terms culture and care or caring need to be defined separately. Culture, defined in the discipline of anthropology, represents two main conceptualizations: behavioral and cognitive. Behaviorally, Harris (1968) defines culture as the behaviors, customs, and way-of-life observed by a group. Leininger (1995c) defines culture as patterns of behavior that are shared among the members of a group and spread to others over time. Nurses in a group caring for patients have specific behaviors in the group. Observations and descriptions of behaviors explain how and why they do what they do. Cognitively, Roper and Shapira (2000) define culture as a group of rules for living that are learned. These rules tell group members how to behave, what to eat, and how 
to respond to the world around them (p. 3). Nurses, as a group, learn how to care for patients, how to respond to their needs, and how to act as they learn the rules exemplified by the group. For the purposes of this paper, culture was defined in a behavioral (patterns of behavior and values shared by a group and transmitted to others in the group through time) and cognitive manner (rules that tell group members how to behave and how to respond to the world around them) because the nursing behaviors, which will be observed, will represent both dimensions of culture.

The concept of caring is considered central to nursing (Watson, 1979, pp. 8-9). Although the term caring is difficult to define because the experience of caring is personal, many nurse theorists have added to nursing knowledge through their theoretical work on the concept of caring (Boykin \& Shoenhofer, 1990, 1993; Leininger, 1978, 1983; Watson, 1979). As Boykin and Shoenhofer were revising the caring-based curriculum at the College of Nursing at Florida Atlantic University, they recognized the importance of conceptualizing the meaning and purpose of nursing. During these revisions they identified the focus of nursing as "nurturing persons living caring and growing in caring” (Boykin \& Shoenhofer, 1993, p. 22). This intention of nursing to care for others guided the idea of nursing as a shared, lived experience called "caring between.” When the nurse enters the world of the one being cared for with the intention of being a caring person, this encounter is called “caring between,” which cultivates personhood (Boykin \& Schoenhofer, 2001a). Without the “caring between,” nursing in its fullest expression does not occur. With an individual patient the nurse enters the patient's care with the intention of caring for the patient. When the nurse knows the patient and cares intentionally, this knowing affects the nurse's response and transforms the knowing from general to specific (Boykin \& Schoenhofer, 2001a). The nurse response develops and evolves over time as the acts of caring 
are practiced and understood through multiple patient care experiences. Boykin and Shoenhofer's theory of Nursing as Caring assumes: (1) to be human is to be caring, and (2) the purpose of the discipline and profession is to come to know persons and nurture them as persons living caring and growing in caring (Tomey \& Alligood, 2006, p. 414).

Another nurse theorist, Leininger, who is also a cultural anthropologist, defines caring as an abstract expression of "assistive, supportive, enabling, and facilitating ways to help self or others with evident or anticipated needs to improve health, a human condition, or a lifeway, or to face disabilities or dying” (as cited in Tomey \& Alligood, 2006, p. 478). Leininger’s theoretical work within the ethnonursing research method combines the concepts of caring and culture to construct the term cultural care. Cultural care can be translated into two distinct kinds of care: transcultural nursing, in which a nurse specialist practices, teaches, and performs research for a select or distinct culture; and cross-cultural nursing, as nurses using anthropological concepts in nursing practice (Leininger \& McFarland, 2002a). Although nursing literature and nursing research mention the terms cultural care, transcultural nursing, and cross-cultural nursing, none of these terms and definitions encompass the definition of culture of care for this research.

Watson's theory of human caring (1988) provides nurses with 10 carative factors to guide nurses in intentional and holistic care of patients. According to Watson (1985a) caring is a term to describe how nurses give care to patients. Responding to each person as a unique individual, the caring nurse perceives and responds to the specific needs of patients (Watson, 1985a). Therefore, Watson's definition of caring depicts the nurse providing individualized care to patients. For the purpose of this paper, care or caring will be defined as intentional and holistic care to infants. 


\section{Patient-centered Care}

Because a common use of focused ethnographies is to explore nursing units (Roper \& Shapira, 2000) that are placed within a larger context of a hospital system, it is important to understand how the hospital system views culture. "Patient-centered care" has become a term to describe individualized holistic patient care in the last decade (Agency for Healthcare Research and Quality, 2001; Institute of Medicine, 2001, 2010; Picker Institute, 2014; Robert Wood Johnson Foundation, 2013). According to the Institute of Medicine (2003), on providing patientcentered care, healthcare providers "identify, respect, and care about patient difference, values, preferences, and expressed needs; relieve pain and suffering; coordinate continuous care; listen to and continuously advocate disease prevention, wellness, and promotion of healthy lifestyles, including a focus on population health” (p 45). The AAP (2012a) states that patient-centered care improves the health outcomes of pediatric patients, increases patient satisfaction, increases professional care-giving satisfaction, and is cost-effective (p. 397). Patient-centered care has become one of the six improvements encouraged by the Institute of Medicine (IOM) to decrease the poor outcomes of healthcare reported in Crossing the Quality Chasm: A New Health System for the 2st Century (IOM, 2001). One recommendation from the IOM (2010) is for healthcare institutions to "support and help nurses in taking the lead in developing and adopting innovative, patient-centered care models” (p. 2). Maizes, Rakel, and Niemiec (2009) state, regarding healthcare team members participating in patient-centered care: “A primary health partner, who knows the patient well, is able to address mind, body and spiritual needs, and coordinates care with the help of a team of practitioners is at the centerpiece. Collectively, the team can meet all the health needs of the particular patient and forms the patient centered medical home” (p. 2). 
Hospital systems have focused on patient-centered care as the main provision for quality care for their patients. Mountain States Healthcare Alliance (MSHA), a 13-hospital integrated healthcare delivery system based in Johnson City, Tennessee, received the 2012 National Quality Healthcare Award from the National Quality Forum, recognizing its achievement of numerous quality-focused goals. These goals to provide quality care to patients represent "providing the best care possible to the people of our region" (Becker's Healthcare, 2012, 10 Guiding Principles for Patient-Centered Care section, para. 2). Some of MSHA’s 10 Guiding Principles for PatientCentered Care consist of: all team members are considered caregivers, care is customized and reflects patient needs, care is provided in a healing environment of comfort, peace and support, and all caregivers cooperate with one another through a common focus on the best interests and personal goals of the patient (Becker Healthcare, 2012, 10 Guiding Principles for PatientCentered Care section, para. 1-10). These intentional and holistic care goals for patients reflect more than a philosophy of healthcare, but concrete decisions for care.

Also connected to hospital systems, in regards to culture, is Duke University Medical Center's emphasis on a culture of safety. The development of the culture of safety originates from the IOM's Crossing the Quality Chasm: A New Health System for the 2st Century (IOM, 2001), which has an emphasis on six areas of safety; assessing the culture, teamwork, patient involvement, systems, openness or transparency, and accountability (Duke University Medical Center, 2005, Overview section). Duke University Medical Center's approach to culture and safety is that each discipline and each unit in the hospital need to be assessed for the current culture of safety that exists in order to change the culture to one of safety for all patients. The implementation of tools and processes to consciously change a culture will promote a culture of safety (Duke University Medical Center, 2005, Summary section). The examination of a current 
culture is how changes can be made to a culture to provide best outcomes for patients. The term "culture" in this hospital center has a focus on "safety," which has been the result of intentionally describing how and what has been going on to arrive at an intentional place of safety for patients.

Sharp HealthCare in San Diego, California is another example of an institution that's desires and goals for healthcare stem from quality care to all patients. Along with safe care, Sharp HealthCare strives to provide a culture for effective, patient-centered, timely, efficient, and equitable healthcare. In regards to the work environment or culture, Sharp focuses on teamwork, compassion, and creating healing environments as they care for patients in an atmosphere of camaraderie (Sharp HealthCare, 2007, Patient Experience section). Culture, described by hospitals, consistently employs the terms patient-centered, safe, teamwork, compassionate, and an atmosphere of safety.

Outside of the hospital experience many businesses and institutions have developed a culture of care in their services. Doubletree hotels by Hilton have established a caring program called “Create A Rewarding Experience (CARE).” Words that Doubletree uses to exemplify care are: real, attentive, cheerful, flexible, thoughtful, honest, and caring (Doubletree by Hilton, 2014, Our CARE Culture section, para. 2). Indiana University Bloomington has instituted a student-led, staff-supported culture of care program on the Bloomington campus to promote a community of care for one another. This program was initiated to establish a culture where “mutual respect and compassionate action are the norm” (Indiana University Bloomington, 2014, Vision section, para. 2). Doubletree hotels and Indiana University have adopted and instilled a culture of care that is descriptive and tangible for their leaders to implement.

Healthcare institutions and institutions outside of the hospital setting are concerned about creating a culture of care in order to create an environment of care to its clients. Specifically, the 
hospital setting's current emphasis is on patient-centered care. Patient-centered care is the hospital setting's method of providing culture to patients; therefore, culture of care and patientcentered care are linked.

\section{Summary}

Little research exists for nursing interventions for infants with NAS. Nonpharmacological care is crucial to the decreasing of withdrawal symptoms in infants with NAS. Providing excellent nursing care to infants with NAS requires clinical knowledge, patience and commitment (Marcellus, 2007). The empirical research posed five nonpharmacological nursing interventions that require more research: environment (light, noise, and touch), adequate rest and sleep (use of swaddling, nonnutritive sucking, rocking, positioning), feeding (formula and breastfeeding), Finnegan scoring by nurses, and nurses as caregivers (Appendix A). Interventions used for the care of premature and sick infants in the NICU may or may not be the exact interventions to be used for the care of the fragile infant with NAS. Environmental and rest or sleep research is considerably older or nonexistent and needs current research in order to accurately use these interventions with infants with NAS. Although the areas of feeding, Finnegan scoring by nurses, and nurses as caregivers present the most current research, the volume of research is scant. While the gaps in nursing knowledge for the nonpharmacological care of infants with NAS are present, nurses can research these interventions in clinical practice in order to provide evidence-based practice for care.

Clinical Practice Guidelines (CPG) offer a standardized approach to the management of infants with NAS. Sarkar and Donn's (2006) national survey on the clinical management of NAS reports that only $54.5 \%$ of respondents have a written policy for NAS infants' care. Hudak and Tan (2012) recommend that each nursery should develop and follow a standardized plan for the 
evaluation and treatment for infants with NAS showing signs of withdrawal. Murphy-Oikonen et al. (2012) compared hospital data in Canada from infants born 6 months prior to and infants born in the 6 months following the implementation of CPG with significant results. Decreases in Finnegan scores, decreases in the number of measures taken over time, and decreases in LOS were reported for the CPG intervention group (Murphy-Oikonen et al., 2012). For newborn nurseries and NICUs, the National Association of Neonatal Nurses (NANN) has prepared guidelines for writing CPG (NANN, 2009) and The AGREE Collaboration Project has developed an instrument for assessing the quality of CPG (The AGREE Collaboration, 2003) Standardized approaches to the nonpharmacological and pharmacological treatment of infants with NAS can provide evidence-based practice to nursing care.

Nursing interventions are part of a culture of care that nurses provide to patients. The nonpharmacological interventions are learned from other nurses in orientation, training, and ongoing educational opportunities. These interventions are also learned in the how and why of providing care to infants in a culture sharing experience. The purpose of this focused ethnographic study was to identify and describe the culture of care and nonpharmacological nursing interventions performed by NICU nurses for infants with NAS at a children's hospital in the southeastern United States. Exploring this culture of care by participant observation, individual interviews with nurses, and the examination of existing documents will provide a rich description of the culture of care for infants with NAS. 


\section{CHAPTER 3}

\section{METHOD}

\section{Introduction}

This chapter is a description of the qualitative method of focused ethnography. This method was chosen because there is a focus on a distinct population in a specific context. Focused ethnography allowed the researcher to articulate research questions before going into the field with the expectation that the knowledge would be useful for nursing practice (Roper \& Shapira, 2000). Focused ethnography was also congruent with the research purpose. This chapter explains ethnography, focused ethnography, delimitations, gaining entry and recruitment, participants, setting, definitions of common terms, a fictitious example of a typical day in NICU 3, data collection, and data analysis. This chapter ends with trustworthiness of the data and ethical considerations.

\section{Qualitative Research}

This qualitative inquiry was an exploration of the culture of care of NICU nurses caring for infants with NAS. Qualitative research, instead of quantitative research, was chosen to explore meaning instead of measurement. Creswell (2007) states, "We conduct qualitative research because we want to understand the contexts or settings in which participants in a study address a problem or issue. We cannot separate what people say from the context in which they say it” (p. 40). A focused ethnography was used as the study method. This inductive approach allowed for a rich and thick description of this unique culture of care. A focused ethnographic approach was appropriate to explore the culture of care and nonpharmacologic nursing interventions performed by NICU nurses who provide comfort to infants with NAS. This approach provided a holistic interpretation of the knowledge, beliefs, and behaviors that underlie the nursing interventions of the NICU culture of care. Infants with NAS are increasingly being 
cared for in the inpatient hospital setting by NICU nurses. Interventions used for the care of premature and sick infants in the NICU may or may not be the exact interventions that are used for the care of the fragile infant with NAS. Research studies on the nonpharmacological nursing care of infants with NAS encompass five main areas of practice: environment (light, noise, and touch), adequate rest and sleep (use of swaddling, nonnutritive sucking, rocking, and positioning), feeding (formula and breastfeeding), Finnegan scoring by nurses, and nurses as caregivers. The purpose of this ethnographic study was to identify and describe the culture of care and nonpharmacological nursing interventions provided by NICU nurses for infants with NAS at a children’s hospital in the southeastern United States.

\section{Ethnography}

Ethnography is a "process of learning about people by learning from them” (Roper \& Shapira, 2000, p. ix). The goal is to understand why "a group of people do what they do" in a given context (Spradley, 1980). Aamodt (1991) describes ethnography as a way of collecting and analyzing the ways that humans make meaning of their lives by the knowledge and behaviors that are used in their culture. Within the context of nursing Leininger (1996) uses the term ethnonursing to describe the beliefs, values, and meanings of patients by nurse researchers. Ethnography can provide understanding of health behaviors among patients and offer problemsolving strategies for nurses (Aamodt, 1991).

The researcher’s “insider perspective,” called the emic view, allows the investigator to experience the day-to-day-events within the culture in order to see the world from their perspective. The researcher's “outsider perspective,” called the etic view, allows the investigator to maintain professional distance in order to effectively describe and analyze what is learned about a culture (Wolcott, 1999). Fieldwork through participant observation allowed the 
researcher to actively listen to conversations, watch interactions, learn the meanings of language, and discover complex systems in order to make sense of the world the group members interact, work and live (Roper \& Shapira, 2000; Spradley, 1980).

Historically ethnography is a qualitative method that has its roots in the field of anthropology. The product of anthropology is the study of a culture; an ethnography. Ethnography as a scientific method is the work of describing culture (Spradley, 1980). Contemporary ethnography began in the late $19^{\text {th }}$ century as a way to study cultural differences that deviated from Western society's norms and culture. Atkinson and Hammersly (1994) found that two significant events influenced the development of ethnography in the $20^{\text {th }}$ century; the work of Boas, Makinowski, and Radcliffe-Brown - who were committed to the idea of anthropology as a science - and the influence of the Chicago school of sociology. Boas, Makinowski, and Radcliffe-Brown in their detailed descriptions of other cultures, allowed for empirical investigation over speculation (Atkinson \& Hammersley, 1994). The Chicago school represented a foundation for field research in the urban setting viewing the city as a "social laboratory," and the idea of "going native” was expanded to include local significant social groups (Woods, 1992). Therefore, ethnography is no longer consigned to "exotic cultures in faroff places” but is now a means for understanding ourselves, modern cultures, and distinct groups (Spradley, 1980).

Qualitative researchers conduct research on cultures in order to report the multiple realities of a culture. This subjective nature of reality (ontology) is discovered as the researcher gets as close to the participants as possible and uses quotes and themes in the participant's own words to provide "evidence of their different perspectives" (Creswell, 2007). The longer researchers spend time with participants in the field, the more they "know what they know" from 
collected data (Creswell, 2007). Becoming an "insider" allowed the researcher to gain knowledge of the participants (epistemology) and understand their culture. Axiological assumptions in qualitative research are that the research is value-laden, which freely admits the place of bias in the researcher's interpretation of participants. The roles of values are to be acknowledged and written into the narrative (Creswell, 2007). The language of a qualitative study (rhetorical) is the use of a personal voice using qualitative terms and definitions (Creswell, 2007).

As nursing practice has expanded so have the research methods used to study the meanings of health, illness, nursing, and culture (Roper \& Shapira, 2000; Streubert \& Carpenter, 2011). Nurses have used ethnography to study the nature of attachment in a NICU (Bialoskurski, Cox, \& Hayes, 1999), cancer units (Germain, 1979), and children’s quality of life after heart transplantation (Green, McSweeney, Ainley, \& Bryant, 2007). Because ethnography is an approach to understanding human behavior, nursing research of nursing practice helps nurses to understand specific cultures of healthcare. Ethnographic research within a specific subgroup of healthcare can provide improved interventions to enhance the health care strategies already in use by the group.

Triangulation. Triangulation is a method that was first advocated by Denzin (1978) as a way to enrich trustworthiness in qualitative research. Triangulation usually involves the use of multiple methods to corroborate findings (Roper \& Shapira, 2000; Wolcott, 1999). Incorporating triangulation in all field-based qualitative research endeavors gives researchers the advantage of "having more of a look around” in the research setting (Wolcott, 1999, p. 220). Because exact descriptions of a culture may be unclear, looking at data from a different vantage point allows the researcher additional data to make the description more clear. The researcher will compare 
descriptions with published literature, which is limited. In addition, a consultant who is considered an in expert in NAS research will review the descriptions. The application of multiple approaches to ethnographic research (participant observation, interviews and the use of existing documents) allows researchers to confirm findings and conclusions (Streubert \& Carpenter, 2011).

Participant observation. Participant observation is the part of ethnographic fieldwork where the researcher is "learning from people” (Spradley, 1980). Although participant observation is also part of grounded theory and phenomenological research, it is the central strategy in ethnographic research (Roper \& Shapira, 2000). This gathering of data by participating in the daily lives of the members of a group allows a sense of discovery that cannot be obtained by interviews alone. Active involvement in the natural setting allows an outsider to become an insider to experience a culture (Roper \& Shapira, 2000; Spradley, 1980; Wolcott, 1999). The observation of the nurses in NICU 3 was overt participant observation. Overt observation results in participants being aware that they are being observed and covert observation results in an unawareness of being observed. Ethically, many researchers feel that if participants know they are being observed, their behavior will change (Streubert \& Carpenter, 2011).). Clarke (1996) highlights the need to describe an “uncontaminated picture of the unit” (p. 37). Also, some feel that deception in the form of covert observation is necessary to obtain valid and reliable data (Punch, 1994). Overt participant observation was demonstrated by the participants' awareness of the purpose and explanation of this research as an observation and description of the culture of care of nurses who care for infants with NAS. Although the participants may have changed some of their behaviors in the presence of the researcher, there was decreased suspicion of the researcher's motives for the study. Although anecdotal notes 
were taken during participant observation and may have appeared as a disadvantage, this note taking was explained as part of the research process.

According to Spradley (1980) there are three kinds of observations: descriptive, focused, and selective. Descriptive observations start in the beginning stages of participant observation and provide an overview of the culture and initial descriptions of what is going on. Focused observations provide a concentrated look at specific interventions done by nurses in the culture. Based on focused observations, a selected observation of a few nurses, or selected interventions lead the researcher to more in-depth knowledge and findings (Spradley, 1980; Streubert \& Carpenter, 2011). This observational process was not linear but was cyclical in nature. A selected observation may lead the researcher to a focused observation that was not noticed earlier were in the research. It is important for the researcher to be aware of this cyclical process in order to provide a rich comparison of data (Streubert \& Carpenter, 2011).

Participant observation allowed the ethnographer to observe and participate in the activities and culture of group members. This participatory experience permitted the researcher to see the patterns of a culture while building rapport and trust within the culture. Interviewing, both informal and formal, allowed the researcher to discover more in-depth meanings of patterns of behavior for group members. Existing documents in the form of charts from monthly unit meetings, admission data, discharge instructions, written reports, and brochures provided additional meaning to the data. In ethnography the researcher is the instrument; therefore, the data from observations was documented in field notes and interview data were recorded and transcribed. When the data were analyzed, meanings emerged from the data. Analyzing the field notes and transcribed interviews revealed thick descriptions of the examined culture and answered questions proposed by the researcher. The documented description and analysis of data 
is called an ethnography (Roper \& Shapira, 2000).

Key informants. Key informants, or gatekeepers, were participants in the culture or surrounding the culture who may have the power to give or not give permission to make observations or conduct interviews (Spradley, 1980). The early identification of key informants within the cultural setting was paramount to the success of the study. Care was taken to protect the rights and interests of key informants. Key informants may not want to be known as "key informants;” they may or may not share the interests or opinions of the rest of the participants being studied. It was important for the researcher not to exploit key informants and to provide a “fair return” for their role. Some key informants may need monetary compensation or a copy of the written ethnographic record for their role. Ethically, the researcher needed to protect the privacy of informants by maintaining privacy with demographic data and interview data. No names of individuals or the setting was used in the written ethnography, and all written fieldwork documents and reflective journals were stored in a locked box. Any data that were transferred to a computer document was stored on a password-protected computer. Interviews took place in a private room without visual access to the NICU 3 unit to ensure confidentiality for the participants during the interview. The researcher did not provide names of participants who were interviewed to other participants. Informed consent documents were explained and provided to each participant (Appendix 1). The researcher remained trustworthy and treated each key informant and participant in an authentically kind manner (Roper \& Shapira, 2000; Spradley, 1980). Specifically, no harsh or inappropriate words were used during conversations and interviews with participants. Also, if a participant inquired as to what another participant had stated in a previous interview or conversation, the researcher respectfully declined an answer and explained the confidential nature of each participant's contribution to the research. 
Interviewing. Interviews are used in ethnographic research to validate observations and to give direction for future observations (Roper \& Shapira, 2000). Although participant observation can be passive and active, interviews are intentional, inquiring, and require an active asking on the part of the researcher. Interviewing can pose a dilemma for researchers because the interview process can interject the researcher's agenda into the cultural setting. There was no way to know if the questions asked would be answered naturally at a later date or if the questions would never be asked (Wolcott, 1999). Informal interviews were not scheduled but involved asking questions about events or experiences immediately after they occurred. This timing of asking questions after events was crucial to checking participant's perceptions against the researcher’s perceptions (Roper \& Shapira, 2000). Specifically, during a nursing intervention or after a nursing intervention, questions were asked as to why and how the intervention was done with the individual infant. Also, when nurses collaborate on what is to be done to comfort an unusually fussy infant, questions were asked about the process nurses use to find interventions that help comfort infants. This informal interview process preceded formal interviews and helped the formulation of wording for guiding interview questions.

Interviews should last until saturation is reached. Creswell (2007) defines saturation as a point when no new information can be added to the data to bring more understanding. (p. 1240). According to Morse (1994) saturation is when the repetition and confirmation of collected data is reached. The repetitive nature of findings reveals to the researcher that saturation has been achieved (Streubert \& Carpenter, 2011). Although each research setting will require different time in the field, the research question will influence the amount of time in the setting when the research question has been answered and no new data are revealed. When the data are saturated (answers to interview questions are revealing no new information), it is time to end interviews 
(Roper \& Shapira, 2000).

Asking good questions and being a good listener were keys to descriptive interview data (Yin, 2009). Because few ethnographic studies finish as planned, it was crucial for the investigator to maintain flexible and adapt to unanticipated events that occurred in the research setting (Wolcott, 1999). Continuing to have a firm grasp on the issues and the research questions allowed the researcher to stay on target. As each interview was transcribed and analyzed, the researcher interpreted the information and assessed if additional evidence is needed. This inductive approach allowed for the emergence of meanings and for modification and adaptation of interview questions. It is imperative that the researcher be open to contradictory findings. The researcher reporting early findings that may be contradictory in nature to a few select colleagues can reduce bias (Roper \& Shapira, 2000).

Examination of documents. Existing documents may take the form of maps, policies, procedures, biographical data, charts, graphs, and census figures and may provide data that are useful to the research question (Roper \& Shapira, 2000). Permission was needed to acquire some documents; therefore, identification of this process of permission early in the research ensured the use of these documents. The use of existing documents, along with participant observation and interview data, added to the depth of understanding of the culture of care to assure data trustworthiness and confirmability (Denzin \& Lincoln, 2000).

\section{Focused Ethnography}

When nurse researchers decide to use ethnography to study a culture of healthcare, a decision is to be made whether they will conduct a traditional or focused ethnography (Morse, 1991). A traditional ethnography is concerned with broadly defining a culture (e.g., an entire healthcare facility), whereas a focused ethnography study is focused on a smaller group or 
culture (e.g., a specific unit within the healthcare facility) (Morse, 1991). Another term for focused ethnographies, micro-ethnographies, described by Richards and Morse (2007), are useful for evaluating information on a specific topic or a mutual experience of nurses. Leininger (1985) describes focused ethnographies with the comparative terms mini-ethnographies (a smaller focused study of a problem or distinct culture) and maxi-ethnography (a comprehensive study of the general descriptions of a culture) (p.35). All focused ethnographies (mini ethnographies, micro ethnographies, and focused ethnographies) are studies that focus on a distinct problem within a specific culture. Knoblauch (2005) confirms that focused ethnographies are used to focus on a small element of society for a short but intensive time and require an intensive analysis of data. Focused ethnographies are useful to answer questions that are articulated before fieldwork. The learned knowledge is expected to be useful for nursing practice for nurses. Because the concentrated observation can focus on specific questions, less time is required in the field to accomplish the data collection (Roper \& Shapira, 2000). As with traditional ethnographies, focused ethnographies employ the same methods of participant observation, interviewing and the examination of existing documents. These three types of information are intertwined to provide a deep understanding of a culture (Roper \& Shapira, 2000). Specifically, focused ethnography can provide a rich and deep understanding of the culture of care and explore any nonpharmacological nursing interventions currently used by NICU nurses to care for infants with NAS.

Multiple focused ethnographies have gone beyond broad cultural descriptions in order to observe and analyze detailed nursing interventions that contribute to a culture of care. Nurse researchers have conducted focused ethnographies on the cultural context of continence care (Lyons, 2007), the cultural context of critical care nurses and their role in the process to 
withdraw life-sustaining treatment (Landreville, 2008), and the experiences of nurses transitioning to the operating room (Brinkman, 2013). Focused ethnographies provide specific descriptions in a specific context. Higginbottom, Pillay, and Boadu (2013) explain focused ethnographies as a "Focus on cultures and sub-cultures framed within a discrete community or phenomenon and context, whereby participants have specific knowledge about an identified problem” (p. 2). The characteristics of focused ethnographies are specific aspects of the field are studied with a purpose, the background knowledge usually informs the research question, there are purposeful field visits using particular timeframes or events, and data analysis is often with recording devices (Higginbottom et al., 2013, p. 3).

Setting. The study was performed at a children's hospital in the southeastern United States. This healthcare facility is a 152 bed, private, independent, not-for-profit pediatric medical center. The NICU is composed of three separate units that collectively are called the NICU. NICU 1 is the main unit where all infants are admitted. Severely premature infants, infants with the highest acuity, infants with NAS, and critically ill infants remain in this NICU 1 setting until they are stable enough to be transferred to NICU 2 or NICU 3. NICU 2 is a unit for infants to feed and grow in open cribs, infants with NAS, and for the first steps of discharge teaching and instructions to be given to parents before their infant can go home with them. After an infant with NAS has been admitted to NICU 1, the infant may be transferred to NICU 2 or NICU 3. If there are available rooms in NICU 3, this is the first choice for the transfer of an infant with NAS. Previously, the infants with NAS were discharged to the home setting to withdrawal under the care of their mother or parents. After multiple readmissions of infants with NAS for withdrawal care, the decision was made to have all infants with NAS withdraw from maternal substances in the NICU. The increased numbers of infants with NAS remaining in the hospital 
setting after birth or transferred from other healthcare facilities required increased inpatient space for the care of these infants. All three of the NICU settings that comprise the entire NICU require that each nurse have extensive NICU training and orientation. Nurses are taught how to care for the newborn with minor health problems to the most premature infants. Also included in the NICU training is the education of how to care for the infant with NAS. Although NAS infants are located in each of the three NICU settings, the NICU 3 setting is exclusively for infants with NAS. This 16 individual-bed unit admits only infants with NAS. Also, most of the scheduled nurses who care for the infants with NAS in NICU 3 are part of a "core" of nurses who have chosen to care for these infants. At times a NICU nurse from NICU 1 or NICU 2 may be asked to "float" to the NICU 3 because of acuity and census issues; however, most of the NICU 3 nurses prefer to care for infants with NAS.

The 16-bed unit for infants with NAS is located on the third floor and is appropriately called NICU 3. This locked unit has a nursing station and medication room in the middle with rooms divided along three hallways; rooms 1 to 7,8 to 13, 14 to 16 . Each 12-hour shift has six RNs/LPNs, two or three PCAs, and a unit secretary. Although the unit is locked, a security officer walks through the unit every 15 to 30 minutes to provide a secure presence on NICU 3 . When parents come to the unit to be with their infant, the parent(s) use the intercom system outside of NICU 3 to state their name and band number. There is a security camera outside of the unit as well so that the unit secretary can see the parent(s). Once the unit secretary has opened the door, the parents sign in and show their wristbands with a number that matches the infant's band at the counter by the unit secretary. Parents sign out at the same location when they leave NICU 3. 
Five of the RNs or LPNs are usually given three infants to care for in rooms that are next to each other (pod). Proximity of the rooms allows the nurse to hear the infants when they are fussy or crying and allow for quicker access to the needs of the infants. Most nurses do their charting within this three-room area in order to be available to their infants for the day. One RN or LPN is assigned as the resource nurse for the day. This nurse is given one infant to care for in one room, which is closest to the nurse's station, and is the nurse who will cosign for medications with most of the nurses during the shift.

The NICU 3 nursing interventions include medication administration, Finnegan scoring, feeding, teaching, swaddling, comforting, bathing, and routine newborn care for the infant with NAS. NICU 3 was an appropriate setting for this focused ethnography because it has a distinct culture of care within the entire NICU and its exclusive care of infants with NAS.

Sampling. Quantitative research uses the term subjects to inform research, while qualitative research uses the term participants or informants (Streubert \& Carpenter, 2011). Individuals in an ethnographic study play an active role in the research and are referred to as study participants. Participants’ first-hand experiences within the culture being studied provide the data to be described for meaning. The sample of participants was not randomly selected as in quantitative research but was purposefully chosen because of the individual's involvement in the culture. The participants have specific knowledge of the culture and were chosen for the purpose of sharing that knowledge with the researcher (Roper \& Shapira, 2000; Streubert \& Carpenter, 2011).

The core team of nurses. The nurses who comprise the "core" team of nurses in NICU 3 are all NICU nurses. Most of the core team has had years of experience as NICU nurses who care for premature or sick infants. Previous to the opening of NICU 3 for infants with NAS, 
these infants were cared for in NICU 1, NICU 2, or in a set of rooms next to each other on another floor. All NICU nurses have taken care of infants with NAS during their nursing careers. Nurses were randomly assigned to care for these infants by the decisions of the charge nurses on each shift. Some of these nurses were drawn to and enjoyed caring for these infants more than other nurses. When the plan was made to establish an NAS unit on the third floor, nurses began to request working there instead of NICU 1 and NICU 2. Eventually, there were enough nurses to comprise a core team of day and night shift nurses. The core team is scheduled to work on NICU 3. From time to time, if there is a shortage of nurses in NICU 1 and NICU 2, a core nurse may need to float to these units to care for premature or sick infants. Also, if there is a shortage of nurses on NICU 3, NICU 1 and NICU 2 nurses may float down to NICU 3 to care for infants with NAS.

The day shift has 13 core team nurses and the night shift has 17 core team nurses who work full-time, part-time, and as needed. Core team nurses practice "primary care nursing” when they take care of the same infants each time they work, depending on the staffing and infants. Currently, there are not enough nurses to cover all of the shifts needed to care for these infants. NICU 3 relies on NICU 1 and NICU 2 nurses to provide enough nursing coverage for this unit.

At times a doctor or nurse practitioner will write an order stating “core team only" for an infant who is not captured and is continuing to need increased dosages of medication. Because core team nurses will be caring for the same infant almost every time the nurse is scheduled to work, the core team nurse will "know the baby" better than a nurse who floats from NICU 1 or NICU 2 and has never cared for the infant. 


\section{Definitions}

Four of the five definitions listed below were common terms used by NICU nurses on NICU 3. These first four definitions were not found in the literature. The last definition, fragile, was a term that is used in the literature but not used by any nurse on NICU 3.

Captured — when an infant is medicated with the highest needed dose of morphine and scores eight or below on the Finnegan score for 48 hours. After an infant is “captured” the infant will begin to be weaned from morphine in small increments:

The definition of captured is when an infant has had their symptoms of NAS under control for 48 hours. This means the infant has had scores of eight or less for 48 hours. At that point we can begin weaning morphine. With that being said, we don't always wait the full 48 hours. Sometimes an infant will have an elevated score in the morning and for the next day and a half, have scores under eight. We won't wait that full next day to begin a wean. Also there have been times when an infant has received an elevated score during the night before the first wean and the scores weren't reported. The infant gets the weaned dose. Generally we just continue the wean (K. Smith, personal communication, July 7, 2014).

Learn the baby — how a primary care nurse describes the process of learning the individual comfort measures needed to provide care and comfort for the infant.

Pod - a set of three rooms next to each other. Each nurse cares for three infants located in three rooms next to each other.

Wean-when an infant starts to be given decreased amounts of morphine for withdrawal. The infant is being “weaned” from the morphine.

Fragile - term used in the literature, but not used by any nurse in NICU 3. This term is 
used to depict the unclear dose-response connection; therefore, these infants can exhibit differing degrees of symptoms (Pritham, Paul, \& Hayes, 2012).

\section{A Fictitious Example of a Typical Day in NICU}

Mary (all names are pseudonyms) has been a NICU 3 “core” nurse for the past 2 years and has just clocked-in at 6:30 am by using the phone at the nurse's station. After putting her purse and lunch in the nurse's break room, she goes to the counter at the nurse's station and retrieves her three "boards” that are each infant's printed information with demographic data, medications, and any pertinent health information. The night shift unit secretary has printed these "boards" for each of the oncoming nurses. Mary then looks for Patricia (the night shift nurse who cared for the same three infants) in order to obtain report on the infant's physical assessment, Finnegan scores, the reasons for some of the withdrawal symptoms scored on the Finnegan score, medications and timing of administration, feeding amounts and types (breastfeeding, expressed breast milk, formula), presence of the mother or father in caring for the infant, any social work issues or concerns, and any techniques used to comfort the infant that were effective. Between the reports for the three individual infants, there is joking about something humorous that was said the day before during work, the sharing of information or questions about the nurse's personal life. Patricia is a NICU 3 nurse who has been in NICU 3 for 3 months who has worked in the NICU for 7 years but has requested to become a "core" nurse in NICU 3. Patricia is still asking questions to other more experienced nurses about providing care to infants with NAS and confirming Finnegan scores to make sure that she is accurately scoring the infant for withdrawal symptoms. After report, nurses document electronically that they have given and received report and have checked the safety equipment in each room. 
Mary then goes into each of the infant's individual rooms to visually observe each infant, monitor setting, and possibly greet any parents who are awake. The pulse-oximeter monitor is alarming for the second infant that Mary observes. The pulse-oximeter probe has lost some of its stickiness and will not stay on the infant's foot, so Mary goes to the medication-stock room to obtain another probe, charges it to the infant, and then applies it to the infant's other foot with a small amount of tape. While Mary was in the medication-stock room, the first infant began to cry and a Patient Care Assistant (PCA) tried to give the infant a pacifier to calm her down and help her return to sleep. Mary sees that the PCA is in the room and quickly goes to check on her third infant who is being fed by the mother in a rocking chair. Mary introduces herself to the mother because it has been a few days since she has seen the mother in the room with the infant. Mary encourages the mother and praises her feeding of the infant with words such as "Wow, he is taking that bottle so well from you."

After Mary has seen her team of three infants, she then takes her medication cart to the medication room and signs out the needed medications for the next 3 hours of care for the three infants. Then Mary takes her cart to the Automated Medication Dispensing cart, located on a hallway in NICU 3, and retrieves the other medications that she will need for the next 3 hours. Mary's cart is only opened by the use of her password and is locked after each use to secure the safety of the medications for the infants. At 7:45 am, Mary goes into the first infant's room to assess the infant, take vital signs including blood pressure, gives the medications ordered, changes the diaper and feeds the infant formula. Mary sits in the patient's room and feeds the infant while talking with and looking at the infant, calling the infant by her first name. After the infant has been fed and burped, the infant is reswaddled and either put on its back in the crib or put in the swing, depending on the fussiness of the infant. The first attempt at laying the infant 
down is in the crib is on his or her back, according to the safe sleep protocol. If the infant continues to be fussy and will not self-soothe, the infant will then be put in the swing or handed to a PCA to rock. Most of the time infants will be given a pacifier to help them sleep. This first infant of Mary's team has almost fallen asleep with her pacifier, so she places the infant in the crib. Mary has a few minutes before the next infant's assessment, so she checks the bedside table for baby linens and is thankful that the night shift nurse stocked it with fitted sheets, cloth diapers, blankets for swaddling, and infant clothes. She will stock each of the bedside tables in the infant's rooms with new baby linen for the oncoming night shift later in the day. At some point during the day, Mary will change the sheets and provide clean blankets and cloth diapers for each infant.

A few of the nurses are leaving the unit to get breakfast from the cafeteria and they ask Mary to join them. Mary lets them know that she cannot leave because her second infant needs to be assessed and fed, but gives the nurses her order for bacon and scrambled eggs. Before the nurses leave for breakfast, one of the nurses walks up to Mary and mentions that this infant needed to be held and gently bounced to calm down when fussy. Then, Mary goes into the second infant's room and the infant has a high-pitched cry, has become unswaddled and has a very smelly diaper. The infant is also slightly tachypnic and is frantically trying to get her fingers in her mouth to suck on her fingers. Mary picks up the infant and tries to soothe the infant by saying her name and telling her that she is going to be okay. Mary stands with infant, gently holding and bouncing the infant for a minute to try to calm her before changing her diaper and assessing her. Mary gives the infant her pacifier and continues to talk to her and hold the infant close to her body. Mary is thankful for the other nurse's recommendation to stand, hold, and gently bounce the infant especially because this intervention helped the infant to become 
calm. When the infant becomes a little calmer, Mary takes the infant's clothes off, changes the diaper, and takes the vital signs including blood pressure. During this entire process Mary is talking to the infant, saying soothing words to the infant, looking at the infant and periodically picking up the infant when needed and providing the pacifier. Because it is 8:45, Mary remembers to score the first infant for withdrawal symptoms using the Finnegan Scoring Tool, which is performed one hour after each feeding. Meanwhile, the third infant begins to scream and Mary sees that a cuddler is walking towards the room to attend to the infant. Mary then returns to the second infant, checks the identification band with the computerized Medication Administration Record (MAR), scans the armband and the medications, and double checks the medications with another nurse in the patient's room. All medications given to these infants require that two nurses check the dosages and sign the MAR. Then Mary administers each medicine to the infant. Mary then asks a passing nearby PCA to obtain two bottles of formula with regular nipples to feed the infant. Mary swaddles the infant and, putting a cloth diaper under the infants chin, feeds the infant the formula. The infant does not eat well and has an unorganized suck. Mary offers chin and cheek support to help the infant grasp the nipple and begin the suck and swallow process. Between sporadic sucking the infant cries out and wiggles hands free from the tight swaddle. The infant has a good rooting reflex but has trouble keeping a grasp on the nipple. Slowly, Mary feeds this infant for 30 minutes and burps her. While the second infant is being fed by Mary, a PCA comes into the room to let her know that the first infant is fussy and will not calm down. Mary asks the PCA if she has time to hold the baby until she calms down and then put her in the swing if needed. The PCA goes to the first infant, reswaddles the infant, provides the pacifier, and holds the infant in the room. 
Meanwhile, Mary is still trying to calm the second infant down and get her to sleep, when the infant has an explosive watery stool. Mary changes the diaper and applies barrier cream to the infant's buttocks to prevent future stool from sitting on the skin. Mary tries to place the infant in the crib, but she is fussy and is drawing up her legs. Mary picks the infant up and gently bounces the infant, reswaddles the infant, provides the pacifier, and talks in a soothing voice to the infant for 10 minutes. When the infant is quieter and less fussy, Mary places the infant in the swing and also puts on some soft music for "white noise" and turns the lights down in the room to help the infant to be able to sleep better.

Mary has about 10 minutes before her third infant needs to be assessed so she quickly rewarms her bacon and eggs, drinks some coffee from her travel mug that she brought in 2 hours ago, and goes to the restroom. The third infant is asleep in his crib. The mother who was feeding the baby earlier is not in the room. Mary does not want to wake the infant, but it is time for the infant's medications and feeding. Mary assesses the infant, obtains vital signs including blood pressure, administers the scheduled medications after another nurse cosigns the medications, and calls the mother to see if she wants to feed the infant. This infant is occasionally breastfed by the mother and then supplemented with formula. The mother returns to the infant's room, washes her hands, and sits in the rocking chair with pillows to begin feeding her infant. Mary asks the mother to help her with the diaper change and to put clean clothes on the infant. The mother rises to change the diaper and retrieves clean infant clothes from her overnight bag to put on her son. Then the mother wraps the infant loosely in the blanket, so that the infant will not get too warm while breastfeeding. Mary helps the mother get set up to breast feed with the pillows and is talking with her about her infant's weight gain from the previous day. The mother asks Mary what her son’s “scores” were and Mary provides the answer, “five, seven, six, five” as the 
previous 12 hours of Finnegan scores for her son. After the mother has started breastfeeding the infant, Mary goes to the medication-supply room to obtain a bottle of formula for supplementing after breastfeeding. Mary asks the mother if she has any questions for her, but the mother does not for now.

This first 3 hours of nursing care for infants with NAS is typical. The nurse is going from room to room with the help of a PCA to calm the infants between feedings. Although the infants all have the diagnosis of NAS, they each requires individual comfort and soothing to become calm. At times a nurse may have a very fussy or "difficult” team of three infants and a PCA, a unit secretary or a "cuddler" to help hold the infants in order to calm them. Also, as a nurse "learns the baby" he or she is better able to provide the individual comfort an infant may need. The nursing care of these infants is not linear in that the nurse cares for the first, second and third infant in order and then begins the same order again. There is usually a back-and-forth motion of nursing care among the three infants.

\section{Researcher as Instrument}

When ethnography is the method for research, the researcher becomes the instrument for data collection (Creswell, 2007; Streubert \& Carpenter, 2011). Two kinds of knowledge are observed by ethnographic researchers; tacit and explicit. Tacit knowledge is best explained as the knowledge that members of a culture know but do not talk about openly. Explicit knowledge is the easily observed knowledge of a culture (Spradley, 1980; Streubert \& Carpenter, 2011). The researcher conducting participant observation, formal and informal interviews, and the examination of documents access both tacit and explicit knowledge. In order to gain access to this knowledge, the researcher must understand her role in the discovery of cultural meanings. The researcher becomes the instrument for the discovery of knowledge through participation in the culture, interviewing group members, collecting documents, analyzing the findings, and 
writing the ethnographic report (Streubert \& Carpenter, 2011). The researcher needs to regularly reflect and document her individual impact on participation in the culture (Creswell, 2007).

Conscientiousness of the researcher's presence in the naturalistic setting allowed for the awareness or prevention of bias. It is possible for the researcher's presence and interactions with participants to change behaviors in ways that would not have occurred in the absence of the researcher (Denzin \& Lincoln, 2000). The concept of “observer bias” is recognized as a topic of primary importance to the researcher during ethnographic data collection. Researchers who conduct research in the natural setting bring their biases, talents, personality, and limitations to the research; therefore, it is imperative that the written quality of analysis and writing become the “measure of usable observational data” (Denzin \& Lincoln, 2000, p. 676).

In learning how to be the researcher as a research instrument, introspection was needed. Introspection is an increased awareness of self, responses and emotions regarding what are observed in the culture being studied, and a reflection on how this was portrayed in the cultural setting. Basic to field work is the researcher's self-knowledge of the researcher's own values, ethics, and cultural background and how these are affected by the research and how the researcher’s self affects the research (Morse, 1991). Roper and Shapira (2000) ask excellent questions for the researcher to keep in mind during research: "Does the investigator change the interaction, responses, and behavior of the participants? How does the investigator separate his or her "self" from the data? If it is possible to use the self as data collection instrument--how is it learned?” (p. 12). In addition to field notes, a reflective journal to record the researcher's personal experiences was used to record feelings, differing perspectives, biases, and the state of the researcher on the research. This journal was used after each fieldwork entry in order to process and record the researcher's thoughts and feelings about the research. This reflective 
journal was locked in a storage box with the fieldwork journal in the researcher's home office.

In an effort to understand the culture of nursing within the NICU environment, the purpose of this ethnographic research was to explore and describe the culture of care and nonpharmacological nursing interventions provided by NICU nurses for infants with NAS.

\section{Delimitations}

This study was delimited within the ethnographic framework of allowing the researcher to select members of the culture based on the research questions (Creswell, 2007, p. 128). The purposive sample was chosen in order to observe and describe full-time nurses who care exclusively for infants with NAS. Participant observation was scheduled to begin in May, 2014; however, due to the delay in Institutional Review Board (IRB) approval, observations began in June, 2014. The dissertation chair and the researcher decided in advance that the first week of participant observation would be an "immersion phase" into NICU 3. Because I am a cuddler on NICU 3, a clinical nursing instructor for a university, and on the NAS task force team, it was important for this new role to be distinguished and established. Also, it was decided in advance that participant observation would take place for 2 hours per day for 5 weeks and occur on all shifts. Field notes were to be written within 1 hour after participant observation in order that the details of observation were remembered.

Although there are registered nurses (RN), licensed practical nurses (LPN), and patient care assistants (PCA) who care for these infants, only the RNs and LPNs were interviewed because they are the population of nurses performing most of the nonpharmacological nursing interventions. Also, on NICU 3 only RNs and LPNs are considered core team nurses. PCAs will often float to all three NICUs according to the staffing needs of the unit. The goal was to provide a description of the nurses who delivered the most care for infants with NAS in order to 
gain perspective of the culture of care. Therefore, only RNs and LPNs who worked full time were interviewed.

All participants were recruited from a single children's hospital in the southeastern United States. Therefore, the sample of nurses was homogeneous in their nursing experiences on the unit and with the other health care professionals. This hospital setting was chosen because of its high prevalence of infants with NAS, but even more importantly because NICU 3 is the only known unit of its kind. To date, there is no known NAS specific unit where infants are cared for in the inpatient setting to withdraw from maternal drugs.

Another delimitation is within the ethnographic method and the research question. Describing a culture of care provided by nurses to infants with NAS is lacking in the literature; therefore, the exploration of a limited number of participants in a discrete community allows a single researcher to provide specific descriptions in a specific context (Higginbottom et al., 2013, p. 3). Each of these delimitations was presented to explain the parameters of the research in regards to what I will and will not do. These boundaries offer a protection for the integrity of the research, the method, and the researcher.

\section{Gaining Entrance and Recruitment}

Gaining entrance by cuddling infants with NAS on a regular basis was the first opportunity for exposure of the researcher to the NICU 3 unit. Added to this experience, I am also a member of the NAS taskforce committee, which included many NICU 3 nurses. Approval by the Director of Critical Care and the nurse manager from the NICU also allowed for access to the NICU 3 unit and provided the first levels of acceptance by the nursing staff toward the researcher. In order to clarity my role as a researcher, I did not continue these other roles throughout the research process. 
Before IRB approval and research, I met with the Director of Critical Care and the NICU nurse manager to discuss the possibility of conducting research on the NICU 3 unit. I presented the proposal for a focused ethnographic study of the nurses and the culture of care that they provided to the infants; this was met with great enthusiasm and approval. I then went through the hospital center’s IRB process and East Tennessee State University's (ETSU) IRB process. Before IRB approval, I presented this study to the NAS taskforce committee and to the hospital's Research Council in April, 2014. After IRB approval, an information email was sent to the NICU 3 nurses and a research flyer (Appendix F) was posted in the medication room on NICU 3. The NAS task force meeting, the email, and the research flyer all allowed for the researcher's contact information to be stated in case the nursing staff had questions and to clearly state that participant observation and interviews were optional. The hospital setting's IRB and ETSU's IRB approval was granted in May, 2014. ETSU's IRB requested that I have individual Informed Consent Documents (ICD); one for participant observation and one for interviews.

\section{Participants and Setting}

A purposive sample of NICU 3 registered nurses who are employed by the hospital were observed and interviewed. Inclusion criteria required that each nurse be a NICU staff nurse (RN or LPN), work full-time, provide direct patient care, and predominantly work in NICU 3. Almost all of the nurses were observed during the participant observation period. I found that there were around 30 nurses who worked predominantly on NICU 3. In the first week of participant observation, I discovered that there were 16 core team nurses who worked full-time. All of these full-time nurses were recruited and invited for interviews, with 12 participating in interviews and 4 not available for interviews. 
There were 12 nurses who participated in interviews. There were 11 RNs and one LPN. Nurses ranged in age from 27 to 63 with a mean of 46.1. Four of the 12 nurses had 3 or fewer years as a NICU nurse, with the other nine nurses having greater than 5 years and up to 40 years of NICU experience. All of the nurses interviewed have had at least 1 year of experience in the NICU 3 with five of the nurses who have worked in the NICU 3 since the opening of the unit.

\section{Data Collection}

Participant observation. As stated by Spradley (1980), the researcher will conduct participant observation with two purposes; “to engage in activities appropriate to the situation and to observe the activities, people and physical aspects of the situation” (p. 54). I began participant observation in June 2014 after receiving IRB approval from ETSU and the health care facility. I introduced myself to each nurse and healthcare worker during the first week, which was an "immersion phase” to clarify my role as a researcher. I knew many of these nurses from being a cuddler; therefore I needed to state to each nurse that I was on the unit for research purposes only. I observed nurses for 2 hours each day during day shift and night shift for this first week and took anecdotal notes as needed. I also requested that each nurse sign the ICD for participant observation, which officially began the next week. All nurses I observed readily signed the ICDs.

During weeks 2 through 6 participant observation occurred on day and night shift and during shift change in order to obtain a comprehensive observation of the nursing unit. I was right next to each nurse as I observed each nurse on NICU 3. I would also follow nurses one at a time into the infant's room in order to further observe their actions with the infants. This close observation with each nurse allowed for the asking of questions as needed or to clarify why a nurse did a particular action. Anecdotal notes were taken during the observations in order to 
remember details of the descriptive, focused, and selective observations. Field notes were written within 1 hour after each observation and recorded on a password-protected computer. Initially, I used the SPEAKING (Setting, Participants, Ends, Act, Key, Instrumentality, Norms, Genre) acronym by Hymes (1972), as recommended by Roper and Shapira (2000) to categorize the details of the observations. This process felt constricting and as if some of observation had to be forced into categories. After a few days of writing field notes, I switched from using the SPEAKING acronym to writing observations in a narrative form. The narrative process of writing was more natural and holistic. At the end of the fifth week I evaluated the findings from field notes to determine if more participant observation was needed. No new information was being gathered; therefore, I felt that the participant observation stage could end and interviews could begin.

Interviewing. During the last 2 weeks of participant observation, participants were invited to participate in a 1-hour interview to be conducted in a private and quiet setting, not within view of the NICU 3. Hospital administration and the nurse manager approved this interview setting. Each interview was scheduled with the researcher for a date and time that was convenient for the nurse. The day before each interview, I contacted the participant by phone or email as a reminder for the interview time and place. Semistructured interviews were conducted and each interview was audio recorded. In order to provide consistency and the needed energy for interviewing, no more than two interviews were done per day.

Each interview began with the researcher’s appreciation for the participant's time and involvement in the research process. Each participant signed an ICD for interviews as prescribed by ETSU IRB. Then, each nurse was given a demographic data sheet to fill out. The demographic data form included the name, age, gender, years of experience in the NICU, and 
years of experience in NICU 3. Both of these forms were copied later and the copies were given to the interviewed participant. Also, each interview was recorded on an audio recorder in view of the participant. It was explained that this interview would be transcribed and that this information would be useful for understanding the culture of care provided to infants with NAS. Interviews were audiotaped on two separate machines in order to ensure the recording and to prevent the loss of the interview due to possible mechanical failures. The original ICD for interviews, demographic data sheets, and audio recorder were stored in a locked box in the researcher's home office. All audio files and transcribed interviews were stored on a passwordprotected computer.

Formal interviews require planning and the use of an interview guide. Spradley (1980) suggests starting with more global questions and then transitioning to more specific questions. Interview questions were stated in Appendix $\mathrm{H}$. The 5 weeks of participant observation taking field notes and writing reflective journals helped me to formulate my guiding questions for the ethnographic interview with core nurses from NICU 3. The researcher conducted individual interviews with nurses with the goal of obtaining an account in the healthcare providers own words about the culture of care provided to infants with NAS. Some of the guiding questions that were asked were, "Could you please explain how you came to be a part of the "core team” of nurses who work in NICU 3?”; “Tell me what it is like caring for infants with NAS?” and "Tell me about the type of care that you provide for these infants." More specific questions were, "Are there types of interventions that you have found helpful in soothing these infants?”; “Describe how you help an infant with NAS to get adequate sleep and rest?”; and "What role do you see yourself in with these infants?” The goal of the interviews was to gain deeper understanding of individual perspectives on the NAS unit culture of care for infants with NAS. After each 
interview I wrote a reflection of the interview in the reflective journal. This discipline of reflection allowed the researcher to persist and stay loyal to the research question and focus on the "big picture" of this holistic culture of care for infants with NAS. Also, after each interview the interview was transcribed verbatim. Each transcribed interview was checked with the audio file for accuracy. All transcribed interviews were stored in a locked box in the researcher's home office.

Examination of documents. A physical map of the 16-bed NICU 3 unit provided a picture of the setting of the research. I obtained this physical map from the engineering department; however, this department deleted any names or identifiers from the document. The existing documents revealed a map of the unit (Appendix G) described earlier in this chapter and a notebook titled “Your Baby’s Journey Through the NICU.” This notebook has a list of support services, NICU 3 guidelines for parents, lists of ways that parents can care for their infants, and educational materials. Also, this notebook can be used as a scrapbook of the infant's hospital stay in NICU 3. Scrapbooking materials and art supplies were available on the unit to allow parents to chronicle their infant's growth and progress. Brochures and teaching material in the notebook provided general information about NICU 3.

\section{Reflexivity}

Each day, after participant observation and completion of the field notes, I wrote extensively in the reflective journal about that day's activity. This process of "putting on paper" my thoughts, feelings, questions, and insights all allowed me to put emotional meaning to what I had observed. I did find myself attaching and bonding with some of the babies and wondering about their future home environment after they left the hospital, as many of the nurses do. This reflection on what I observed and experienced proved helpful to the why of my passion for these 
infants. During an interview with a nurse, it was found out that both this nurse and I took care of the same infant in the NICU many years ago. Unresolved grief had been a part of me since the death of this infant. However, the shared memories, shared grief, and shared love for this infant brought much healing to me. Although this unexpected shared experience does not explain all of why in caring for these infants, it did remind me that caring for infants of all kinds has been always been a desire of mine. This experience gave me a different sensitivity to the nurses and the attachments that they make with these infants. After this specific interview, I listened even more intently as they described their "love for a baby" or their concerns about an infant's future, knowing that these feelings for these infants were very deep and impacting on the nurses.

Throughout the research process I assessed how I felt about the specific experiences of participant observer and interviewer. The questions by Roper and Shapira (2000) stated earlier were the focus of the writing in the reflective journal. I had to ask myself if my presence on the unit changed the interactions or behaviors of the participants. All of the nurses shared easily with me their care, thoughts, and feelings about working with infants with NAS and working on NICU 3. After the first week of immersion, I felt very comfortable with these nurses. Although my role as a cuddler was suspended for the length of the research study, some subjective bias occurred due to the previously formed relationships with nurses and staff related to this "cuddler" role. These thoughts and feelings were recorded in the reflective journal. In regards to the question, “How did I separate myself from the data?” I had to continually use introspection to remind myself that I was not one of them. However, many times during the research process nurses asked when I was going to start working with them on NICU 3. Although this was a compliment and a sign of rapport, I had to remind myself of my researcher role. At times there was the tension between researcher as researcher and researcher as a cultural member. I found 
myself becoming attached to infants in much the same way as the nurses. It was difficult to stay distant from the infants while holding infants and being a part of the nurse's care of the infant. Becoming part of the culture was the way to immerse myself in the culture to learn from the nurses; however, I had to deliberately stay connected to my feelings in order to avoid bias and misinterpret the data. I do not think that I could completely be free of bias in this research; however, daily I reflected on this process, asked myself questions and wrote in the reflective journal. This reflective journal was stored on a password-protected computer.

\section{Data Analysis}

The analysis of ethnographic data is both process and product. The process consists of separating raw data into small bits and then reconstructing them into patterns or wholes to make the product of ethnography. This analysis does not happen in a strict linear manner but in a back and forth motion. This process allows for deeper understandings of culture and richer descriptions (Roper \& Shapira, 2000). Roper and Shapira’s framework for the analysis of ethnographic data was used for this research and included: 1) coding for descriptive labels, 2) sorting to identify patterns, 3) identification of outliers or negative cases, 4) generalizing constructs and theories, and 5) memoeing to note personal reflection and insights.

The first step of Roper and Shapira's (2000) method was coding for descriptive labels. First, the field notes from observations and transcribed interviews were reviewed and initial coding began. I wrote the codes on the right hand margin of each transcript and on the field notes. This process of coding was difficult and tedious because I checked the codes three times on different days to ensure consistency. The dissertation chair was sent the coding sheets (Appendix I) to ensure that I was coding correctly.

The second step of Roper and Shapira’s (2000) method was sorting to identify patterns 
Roper and Shapira use the term "patterns" in the same way as Miles and Huberman (1994) use the term "themes." The term "themes" was used from here on to reference "patterns." At this point I created a chart with codes, categories, and themes. The codes were clumped into smaller segments to form categories. The categories overlapped in some areas and I began to see the beginning of possible themes. The categories were sent to the dissertation chair to corroborate the coding into categories. The next step was to further reduce the categories into themes. The dissertation chair suggested that I write all of the categories on small pieces of paper and lay all of them out on a table or post them to a wall. Then I was to start putting the categories into small chunks, which resulted in the found themes. These themes were discussed and agreed upon by the dissertation chair and the consultant who is considered an expert in NAS research.

The third step in Roper and Shapira's (2000) method was identification of outliers or negative cases. There were a few unique observations and experiences of nurses that were not consistent with the emerged themes and they were discussed in the results section. The fourth step in Roper and Shapira's method was generalizing constructs and theories. The concepts of culture (behavioral and cognitive) and the concept of care or caring were identified in the themes and are discussed in the results section. The fifth step of Roper and Shapira's method was memoeing to note personal reflection and insights. This step was performed throughout the research process. Many times I woke up during the night to write down a thought or a question. Also, during participant observation and individual interviews often a memo was written down in order to not forget an idea or item for later inquiry. In the course of driving a car, running, or preparing a meal, I would think of insights that needed to be written down for later reflection. This process of memoeing allowed the researcher to make connections between of pieces of information. 


\section{Trustworthiness}

Close social interaction between the researcher and participants created access to the collection of data of a culture. The conduct, rapport, and relational skills of the researcher helped to develop the mutual trust that was needed for ethnographic research. It was essential for the researcher to develop and continue to cultivate good interpersonal skills and be aware of her strengths and weaknesses.

Guba and Lincoln (1985) have identified four techniques to support the rigor or trustworthiness of a research study: credibility, dependability, confirmability, and transferability. Credibility was established by increasing the probability that credible findings were found. Prolonged engagement in the field and member checking (when participants recognize the findings to be true) provide credibility to the research (Streubert \& Carpenter, 2011). Dependability asks the question how dependable are these results? Triangulation of data (participant observation, interviews, and evaluation of existing documents) contributed to the dependability of the findings. The application of multiple approaches to ethnographic research allowed the researcher to confirm findings and conclusions (Streubert \& Carpenter, 2011). Dependability required credibility (Lincoln \& Guba, 1985). Confirmability is when researchers confirm findings by leaving an audit trail, which is a recording of activities over time in order that another individual can follow the research process (Lincoln \& Guba, 1985). I compared descriptions with published literature, which is limited. In addition, a consultant who is considered an in expert in NAS research reviewed the codes, categories, and themes from participant observations and individual interviews. The audit trail was written in the form of field notes, reflective journal, transcribed interviews, demographic data, and coding that is locked in a secure location or located on a password-protected computer. Transferability was the probability 
that the findings have meaning to other individuals in a similar situation. This provision of a database or process of data collection, if needed, made the transferability of the findings available to other researchers (Lincoln \& Guba, 1985). A detailed and rich description provided meaning of this culture of care so that other NICUs can use these results. After completion of this dissertation, the results will be disseminated and published in a scholarly journal. These four criteria supply rigor to qualitative research to outsiders for the accurate representation of participants’ experiences (Streubert \& Carpenter, 2011).

\section{Ethical Considerations}

Conducting research in the natural setting caused the researcher to be aware of ethical obligations to the participants. Carper's (1978) work on ways of knowing in nursing explains four ways of knowing: empirical knowing, aesthetic knowing, personal knowing, and moral knowing. Moral knowing reflects an ethical obligation to act in a way that is right and just in a given situation. This abstract knowing is based on traditional codes of conduct but is most important when the individual is faced with situations where the decisions of right and wrong are blurred because of value systems and beliefs (Carper, 1978; Streubert \& Carpenter, 2011).

Prior to participant observation the Critical Care Director of the healthcare setting was contacted to explain the purpose of the study and to receive approval and direction for presenting the study to the NICU 3 nurses. After this meeting the nurse manager of the NICU was also be contacted to explain the study. The Critical Care Director and the nurse manager were both consulted for the best strategy and advice on how to inform the participants and begin the study.

Inclusion and exclusion criteria were evaluated for participant observation and the recruitment of nurses to be interviewed. Information about the research study was then conveyed in a unit meeting, a flyer placed in the medication room, and an email to all nurses who work on the 
NICU 3 unit. Nurses were allowed to decline participation in the observation process. Questions were answered at this time and encouraged at any time during the research process. Informed consent documents were explained to and signed by the participants; documents that indicated they could withdraw from the study at any time. Also, each nurse observed signed informed consent documents before the participant observation period. To protect the confidentiality of the participants, each interviewed nurse signed informed consent documents prior to the start of each interview. Ample time was allowed for participants to read and ask if they had any questions about the process. A signed copy was given to each participant prior to conducting the interview. The researcher's contact information and a copy of the informed consent were left with each participant in case the need arose to contact the researcher at a future date.

Participant observation was conducted in NICU 3 and occurred over a 5-week period with multiple observation sessions amounting to 80 hours with the core team of nurses. During this time the researcher observed and wrote field notes, in addition to writing reflective journals after the interviews. Semistructured interviews using the guiding questions were conducted with 12 of the core nurses. Some of the core team nurses could not participate in interviews due to living great distances from the hospital or due to scheduled vacations. Each interview was conducted on the core nurse’s day off from work. Each interview took about 1 hour. All interviews were audiotaped on a digital recorder that was locked in a fireproof box between interviews. Each interview was transcribed verbatim. To further protect the identity and confidentiality of participants, identifying information was removed from all transcripts before sharing them with the dissertation chair. A numeric identifier was used to refer to interviewed participants. All transcripts and recordings were stored on a password protected hard drive or in a fireproof locked box in the researcher's home office. Confidentiality and privacy were also 
maintained by conducting the interviews in a private location outside of view of NICU 3.

Additional ethical considerations in regards to the methodology were establishing rapport and developing trusting relationship with the participants. The researcher was on time for all observations and interviews and thanked the participants when leaving the study site. The researcher dressed in professional attire that was consistent with the cultural setting. The researcher communicated with the nurses in an honest and confidential manner in all matters concerning the NICU 3 unit research. The researcher developed sensitivity in the research field because the researcher's presence may directly or indirectly affect the culture being studied. Engagement in the research setting established rapport and trust between the researcher and participants (Lambert, Glacken, \& McCarron, 2011).

\section{Protection of Human Subjects}

Prior to the initiation of data collection, approval was obtained from the healthcare setting's Institutional Review Board (IRB). IRB approval forms are located in Appendix B. Approval from the healthcare setting's Research Council is considered an optional approval; however, the approval letter is located in Appendix C. Approval from East Tennessee State University's IRB was obtained after the hospital setting's IRB approval and is located in Appendix D (participant observation) and Appendix E (individual interviews). Participants were assured that information shared with the researcher would remain confidential. Also, all participants understood that they could withdraw from the study at any time. Code numbers were used to identify audiotapes and demographic data forms. All audiotapes and demographic forms will be destroyed after 5 years.

Member checking is often used in qualitative research to check for the trustworthiness of the data (Creswell, 2003). Procedurally, member checking is the process of taking a transcribed 
interview document and returning it to the participant who was interviewed to determine if the data were recorded and transcribed accurately. The participants are allowed to discuss, add or delete information, and state if the interview was transcribed accurately (Creswell, 2003; Denzin \& Lincoln, 2000, p 393). However, member checking can also elicit feelings that a participant may no longer have. A story or emotion felt at the time of an interview may not seem relevant, important, or even embarrassing to the participant during member checking (Sandelowski, 1993). Changes in data may lead to further analysis for meaning. Sandelowski (1993) states, "The very act of reading a transcript for accuracy may not only lead the member to provide additional data that have to be analyzed, but it may itself also cause the member to revise his or her views and/or influence events still to be experienced in the course of the study” (p. 6). Trustworthiness was gained when the researcher can demonstrate that the data were collected and reported consciously and ethically; not by transcribing accuracy (Carlson, 2010; McConnell-Henry, Chapman, \& Francis, 2011).

In order to gain trustworthiness of interview data, and after the transcription of interviews and analysis of the data into themes, participants were provided with final themes and a question to each participant (Andrasik, 2014; Miles \& Huberman, 1994). After reflecting on the final themes, each participant was asked the following question: "Would you be able to see yourself in these general themes?” Each participant's response was recorded, with the nurse stating that she could "see herself" in these themes. These data were used to confirm findings or present a negative case. A codebook containing code numbers and names and addresses of informants was stored in a locked file box separate from all other data. At the end of the research study, each interviewed participant received an individual note of gratitude and a \$25.00 gift card as a gift of appreciation for participation in the study. 


\section{Summary}

Roper and Shapira's (2000) method of focused ethnography was an appropriate method

for this research. The purpose of this study was to explore the culture of care and nonpharmacologic nursing interventions performed by NICU nurses who provide care to infants with NAS. The culture of care was observed during participant observation and recorded in field notes. Also, this culture of care was described during individual interviews. In a focused ethnography the researcher goes into the research with some questions already articulated before research begins. The nonpharmacological nursing interventions that encompassed five main areas of practice: environment (light, noise, and touch), adequate rest and sleep (use of swaddling, nonnutritive sucking, rocking, and positioning), feeding (formula and breastfeeding), assessment and evaluation using Finnegan scoring by nurses, and nurses as caregivers were observed during participant observation and described during individual interviews. The findings from participant observation, individual interviews, and the examination of existing documents are presented next. 


\section{CHAPTER 4}

\section{RESULTS}

\section{Introduction}

The purpose of this study was to explore the culture of care and nonpharmacologic nursing interventions performed by NICU nurses who provide care to infants with NAS. Roper and Shapira's (2000) method of data analysis was appropriate for this focused ethnography. The information presented in this chapter described the sources of data from a fieldwork journal, reflective journal, individual interviews, observational charts, and relevant documents and were then presented as cultural themes. The goal of this researcher was to describe the culture of care provided by NICU 3 nurses who care for infants with NAS. Another goal of this researcher was to provide direction to the nurse caring for an infant with NAS; therefore, four of the five nonpharmacological nursing interventions and the culture of care were presented in themes.

\section{Identified Themes}

Findings from participant observations recorded as field notes and transcribed interviews were coded. These codes were clustered into categorizes which revealed five themes that helped the researcher understand the beliefs and feelings of nurses as they provide a culture of care for infants with NAS. These five themes were: 1) learn the baby (routine care, comfort care, environment, adequate rest and sleep, feeding), 2) core team relationships (support, interpersonal relationships), 3)role satisfaction (nurturer or comforter, becoming an expert), 4) grief, and 5) making a difference (wonderful insanity, critical to them). The cultural themes are presented below with subthemes and quotes that support the categories. Four of the five nonpharmacological nursing interventions are also identified within two of the themes. 


\section{Theme 1: Learn the baby}

Nurses repeated the phrase "learn the baby" during participant observation and interviews. This phrase is defined as the process of learning the individual comfort measures needed to provide care and comfort for the infant. Another way that "learn the baby" was explained was the intentional learning of the baby's personality. The process of learning the baby occurs through routine care, comfort care, and the nonpharmacological nursing interventions of environment, adequate rest and sleep, and feeding.

Routine care. Through observations of nurses in NICU 3 and by individual interviews, I observed the following aspects of routine care that can be described as a set of nursing interventions performed every 3 hours with infants. These nursing interventions included a physical assessment, Finnegan scoring 1 hour after feedings, vital signs, a blood pressure if an infant was receiving the medication Clonidine, diaper changing, changing of clothes if soiled, the administration of medications, and feeding. The next routine care, 3 hours later, included all of the above nursing interventions but did not include a full physical assessment. Nurses recorded all of the routine care nursing interventions every 3 hours. At the end of the feeding, the nurse may have needed to hold the infant, reswaddle the infant, provide a pacifier, turn on soft music, or turn down the lights in order to create an atmosphere of rest and sleep for the infant. If the nurse needed to perform routine care on another infant, a fussy infant may be handed to a PCA or to a cuddler. If a PCA or a cuddler was not available, the infant would be placed in the swing to provide a rocking motion to help the infant to sleep (see Table 1 below). 
Table 1

Routine Care Chart

\begin{tabular}{ll}
\hline \multicolumn{1}{c}{ Every 3 hours } & \multicolumn{1}{c}{ Every 6 hours } \\
\hline Vital signs (temperature, respirations, & All 3-hour routine care interventions \\
heart rate, blood pressure if on clonidine) & minus the full physical assessment. \\
Full physical assessment & \\
Finnegan scoring-1 hour after feeding & \\
(to score for sleep) & Vital signs may be taken from monitor \\
$\begin{array}{l}\text { Diaper change-no variation } \\
\text { Change of clothes or bedding if needed } \\
\text { Medication administration }\end{array}$ \\
Feeding
\end{tabular}

Continued observations in NICU 3 revealed two more components to the routine care provided by NICU 3 nurses. After an infant reached the age of 28 days, the infant was not scored for sleep on the Finnegan scoring tool. Nurses stated in observations and interviews that infants experienced more awake states around 1 month of age and scoring an infant for lack of sleep after 28 days could give the infant a higher score for withdrawal than the infant was actually experiencing. Also, the night shift was required to provide additional care in the form of baths, weight, changing of linens, and measurements (See Table 2).

Table 2

\section{Additions to Routine Care}

\begin{tabular}{ll}
\hline \multicolumn{1}{c}{ Greater than 28 days care } & \multicolumn{1}{c}{ Night shift care } \\
\hline $\begin{array}{l}\text { All of routine care and in between care, } \\
\text { but no score for sleep with Finnegan }\end{array}$ & Baths \\
scoring. & Weights \\
Many infants may feed every 3-4 hours & Change all bed linens \\
and may sleep longer periods of time. & Measurements of infants 1 time a week \\
\hline
\end{tabular}

Comfort care. From observations and interviews with NICU 3 nurses, comfort care was observed and described as any nursing intervention used to comfort infants. Between the routine 
care provided every 3 hours for infants, many infants become fussy, irritable, have stomach aches, need a diaper change, need to suck on a pacifier, become unswaddled and need to be reswaddled, need to be held, need to be talked to, and need to be rocked in a swing or by a nurse. Until an infant with NAS is “captured,” most infants have very fussy periods during the day. An infant is considered "captured" when the infant is on the highest needed dose of morphine with Finnegan scores eight and below. However, many infants may still have fussy periods even after they are “captured” and have started weaning from their morphine dosages. Comfort care interventions can be provided to infants with NAS individually or in combinations to help relieve withdrawal symptoms (see Table 3). These nursing interventions were provided during routine care and between routine care.

Table 3

Nursing Interventions Used to Comfort Infants During Routine Care and In-between Care

Swaddling (tight swaddle with blanket, hands covered swaddle, Halo swaddle)

Holding and rocking

Talking/singing to the infant

Rhythmically patting the infant's bottom

Lights off-dark room

Quiet room

Soft music, sound machine, TV off, white noise from sound machine or TV

Looking at infant

Pacifier

Infant riding in a stroller

Infant strapped to a nurse with a front backpack or Moby wrap

Swing

Infant's abdomen on nurse's legs to help with abdominal discomfort

Heel warmer to infant's abdomen over a thin layer of clothing

Hands covered with mittens to protect the skin

Participant 07 described the process of learning the baby as the nurse's intentional understanding of individual infants. 
You know of course we do our assessments and do all those things we're supposed to do, but I guess we look at it more as a whole, meeting their needs and that's different with every baby. Like you can have 10 lined up and they're all gonna have different things that make them feel better or maybe better, positions you feed them or it's learning their habits and I think that's a big plus with core team is you get to know their little quirks. This trial-and-error approach to the nurse's use of nursing interventions for infants with NAS appeared throughout the participant observation period and was stated in the individual interviews. All of the interviews were with core team nurses, and every nurse referred to the fact that core team nurses knew the comfort measures that were needed to care for the infants they cared for. Over time, day after day, the core team nurse learns the baby's comfort needs. Participant 012 described the core team and their understanding of how to comfort their infants. When you have a core team, when we have that group of people, we do this every day. We know what the babies are like. We've learned the babies and whether you know your baby was that way three weeks ago, (you know screaming and peeling the paint off the walls), so you can go and try to help because of what you've done for your baby that maybe calms your baby.

Although many of the nurses in NICU 3 are core team nurses, many interviews and observations revealed that a NICU nurse who "floats" to NICU 3 does not know the baby in the same way as a core team nurse who has cared for the baby for weeks.

Three infants were assigned to an individual nurse each day. A "pod" is defined as a set of three rooms next to each other. The map of the unit supports this finding in regards to the placement of rooms and the assignment of nurses to care for a "pod." When a nurse cares for three infants in close proximity of each other, the nurse can be close to hear and see the needs of 
the infants. Nurses in observations and interviews stated that this assignment of a "pod” allowed them to more easily provide comfort to the infants they cared for, as comfort was needed. Participant 012 described the assignment of groups of babies into pods to increase consistency of care for the infants.

Having people have the same group of babies, 1, 2, and 3; 4, 5, and 6, and sharing your pods with the nightshift person who consistently has these babies because there again, you understand each other. You know I understand what they're talking about, they understand what I'm talking about, we a lot of times will care for the infants the same way so I think that that's worked well.

The next three subthemes of the theme, learn the baby, are three of the five nonpharmacological nursing interventions of environment, adequate rest and sleep, and feeding that were found in the literature. Each of the nursing interventions was described and a chart (Table 4) of how the 12 interviewed nurses performed each of these interventions was provided and explained.

Environment. Of the nurses interviewed and asked the question, "What effect does light, noise and touch have on infants with NAS," all of the nurses stated that all of the environmental stimuli needed to be decreased for the infant with NAS, especially before the infant was captured. Most stated that the infants were "hyper-sensitive" because of the neurological damage from maternal drugs. Participant 09 responded with this reply to the effect of light, noise, and touch on infants with NAS.

If they're un-captured and they’ve just come in, then we keep the shades drawn, we keep the lights out, the noise down because they're very sensitive to all those things and it can start some screaming in such a way that it's hard to get them calmed back down. Now as 
they age, we try actually to expose them to those things because it would not be beneficial to a baby to be in a controlled environment with the shades drawn then (whispering) 'everyone talks really low', and then suddenly you send 'em home with a parent who has three other kids and it’s chaotic, that would just flip them, for they wouldn't be able to organize themselves to do the things that we want them to do, so we start talking to 'em, we turn on the lights, we try to get 'em into a rhythm, you sleep then we turn on the lights and talk and we eat and we feed so they have to learn how to socialize, they have to learn how to deal with the stimuli but it stresses 'em them til they're captured.

In regards to light, all of the nurses stated that the room needed to be dark or darkened for the infants to eat better before the infants were captured. Five of the nurses tried to keep the blinds open with the lights out in the room to keep the pattern of day and night for the infants, but three nurses mentioned that some infants could not handle even this much light.

In regards to sound, all of the nurses stated that the infants were sensitive to noise. Nurses stated that loud noises could startle them, and because they already have a hyper-Moro reflex, a loud noise could stimulate this reflex and in turn make the infant cry. Eight of the nurses mentioned that many infants needed "white noise" to create a small amount of noise to drown out noises from the hallways and from the extra noise created during the change of shift. Some nurses would use the sounds from the swing or use the soft music from the aquarium mobiles in the cribs to provide the "white noise" that may be needed for some infants.

In regards to touch, all of the nurses mentioned the hypersensitivity to touch. Five of the nurses stated that infants "do not like to be touched." Participant 01 stated, "you need to treat the 
babies like they have a 300\% migraine.” Nurses also stated in interviews that the individual rooms for each infant helped to decrease light, noise, and touch.

Culture of care and environment (observational results). To further demonstrate the culture of care provided by NICU nurses to infants with NAS, all 12 of the interviewed nurses were observed for how the nurse decreased or used light, noise, and touch with an infant (Table 4). As a general observation all of the nurses kept the light, noise, and touch decreased. Many of these nurses displayed similar environmental actions with the infant, but there were also some differences.

Most nurses were observed decreasing the light in the infant's room to help keep the infant calm. Two nurses kept the blinds opened during the daylight hours to promote a sense of day and night for the infants. All of the other nurses kept the room dark.

Most nurses were observed caring for infants in a quiet room. Some nurses used the music mobiles on the infant's crib or the white noise sounds from the swing to provide a barrier to the noise that can occur outside of the room and possibly startle the infant. The extra noise that occurred during shift change was observed and stated by the nurses during interviews and observed during participant observation. One nurse observed did not want to use the noise from the television as white noise and another nurse observed did use the noise from the television as white noise. Three of the 12 nurses cared for the infant in a completely quiet room.

Touch was incredibly varied among nurses. Seven of the nurses demonstrated a firm touch or a firm holding of the infant close to the nurse's body. Three of the nurses demonstrated and stated that little or no touching was what the baby needed to remain calm, and two other nurses touched the infant only when needed during vital signs or assessment. Rhythmic patting was a kind of touch that was observed with two nurses but also observed with various nurses 
throughout the observation period. One nurse in particular was observed after an infant's bath. The infant was massaged with lotion while lying prone in the crib, dressed, and held close to the nurse and rhythmically patted. All of the nurses stated that the process of learning the baby allowed the nurse to understand what kind of touch an infant needs to remain or become calm. Table 4

Field Note Observations for Environment: Nurses 1-6

\begin{tabular}{|c|c|c|c|c|c|}
\hline Nurse 1 & Nurse 2 & Nurse 3 & Nurse 4 & Nurse 5 & Nurse 6 \\
\hline $\begin{array}{l}\text { State of the } \\
\text { baby: } \\
\text { stirring }\end{array}$ & $\begin{array}{l}\text { State of the } \\
\text { baby: } \\
\text { awake }\end{array}$ & $\begin{array}{l}\text { State of the } \\
\text { baby: } \\
\text { asleep }\end{array}$ & $\begin{array}{l}\text { State of the } \\
\text { baby: } \\
\text { fussy }\end{array}$ & $\begin{array}{l}\text { State of the } \\
\text { baby: } \\
\text { awake }\end{array}$ & $\begin{array}{l}\text { State of the } \\
\text { baby: } \\
\text { extremely } \\
\text { fussy }\end{array}$ \\
\hline $\begin{array}{l}\text { Light: dark } \\
\text { room }\end{array}$ & $\begin{array}{l}\text { Light: room } \\
\text { dark, blinds } \\
\text { open during } \\
\text { day }\end{array}$ & $\begin{array}{l}\text { Light: room } \\
\text { dark }\end{array}$ & $\begin{array}{l}\text { Light: blinds } \\
\text { open during } \\
\text { day, but } \\
\text { lights off. } \\
\text { Dark room } \\
\text { at night }\end{array}$ & $\begin{array}{l}\text { Light: room } \\
\text { dark }\end{array}$ & $\begin{array}{l}\text { Light: dark } \\
\text { room }\end{array}$ \\
\hline $\begin{array}{l}\text { Noise: white } \\
\text { noise from } \\
\text { sound } \\
\text { machine or } \\
\text { soft music }\end{array}$ & $\begin{array}{l}\text { Noise: } \\
\text { Quiet or } \\
\text { white sound } \\
\text { machine }\end{array}$ & $\begin{array}{l}\text { Noise: quiet, } \\
\text { white noise } \\
\text { from sound } \\
\text { machine }\end{array}$ & $\begin{array}{l}\text { Noise: } \\
\text { white sound } \\
\text { machine, no } \\
\text { TV }\end{array}$ & $\begin{array}{l}\text { Noise: quiet, } \\
\text { white noise } \\
\text { from sound } \\
\text { machine }\end{array}$ & $\begin{array}{l}\text { Noise: white } \\
\text { noise }\end{array}$ \\
\hline $\begin{array}{l}\text { Touch: tight } \\
\text { swaddling and } \\
\text { holding }\end{array}$ & $\begin{array}{l}\text { Touch: firm } \\
\text { holding }\end{array}$ & $\begin{array}{l}\text { Touch: no } \\
\text { touching }\end{array}$ & $\begin{array}{l}\text { Touch: } \\
\text { massage } \\
\text { with lotion } \\
\text { after bath. } \\
\text { Firm } \\
\text { holding and } \\
\text { close } \\
\text { holding. } \\
\text { Some } \\
\text { rhythmic } \\
\text { patting. }\end{array}$ & $\begin{array}{l}\text { Touch: firm } \\
\text { touch, } \\
\text { rhythmic } \\
\text { patting }\end{array}$ & $\begin{array}{l}\text { Touch: } \\
\text { holding and } \\
\text { firm touch }\end{array}$ \\
\hline
\end{tabular}


Table 4 Continued

Field Note Observations for Environment: Nurses 7-12

\begin{tabular}{|c|c|c|c|c|c|}
\hline Nurse 7 & Nurse 8 & Nurse 9 & Nurse 10 & Nurse 11 & Nurse 12 \\
\hline $\begin{array}{l}\text { State of the } \\
\text { baby: } \\
\text { fussy }\end{array}$ & $\begin{array}{l}\text { State of the } \\
\text { baby: } \\
\text { Awake, /off } \\
\text { and on fussy }\end{array}$ & $\begin{array}{l}\text { State of the } \\
\text { baby: } \\
\text { asleep }\end{array}$ & $\begin{array}{l}\text { State of the } \\
\text { baby: } \\
\text { Awake and } \\
\text { quiet }\end{array}$ & $\begin{array}{l}\text { State of the } \\
\text { baby: } \\
\text { asleep }\end{array}$ & $\begin{array}{l}\text { State of the } \\
\text { baby: } \\
\text { fussy }\end{array}$ \\
\hline $\begin{array}{l}\text { Light: room } \\
\text { dark }\end{array}$ & $\begin{array}{l}\text { Light: dark } \\
\text { room }\end{array}$ & $\begin{array}{l}\text { Light: room } \\
\text { dark }\end{array}$ & $\begin{array}{l}\text { Light: room } \\
\text { dark }\end{array}$ & $\begin{array}{l}\text { Light: dark } \\
\text { room }\end{array}$ & $\begin{array}{l}\text { Light: room } \\
\text { dark }\end{array}$ \\
\hline $\begin{array}{l}\text { Noise: white } \\
\text { noise } \\
\text { machine, TV } \\
\text { for white } \\
\text { noise }\end{array}$ & Noise: quiet & $\begin{array}{l}\text { Noise: quiet, } \\
\text { quiet voices }\end{array}$ & $\begin{array}{l}\text { Noise: music } \\
\text { from lullaby } \\
\text { machine or } \\
\text { quiet }\end{array}$ & Noise: quiet & $\begin{array}{l}\text { Noise: quiet } \\
\text { or white noise }\end{array}$ \\
\hline $\begin{array}{l}\text { Touch: no } \\
\text { stroking the } \\
\text { baby, very } \\
\text { little touch }\end{array}$ & $\begin{array}{l}\text { Touch: } \\
\text { holding tight } \\
\text { and still }\end{array}$ & $\begin{array}{l}\text { Touch: little } \\
\text { touch }\end{array}$ & $\begin{array}{l}\text { Touch: } \\
\text { rhythmic } \\
\text { patting }\end{array}$ & $\begin{array}{l}\text { Touch: only } \\
\text { touch when } \\
\text { needed }\end{array}$ & $\begin{array}{l}\text { Touch: } \\
\text { holding firm } \\
\text { and close, no } \\
\text { extra touching }\end{array}$ \\
\hline
\end{tabular}

Adequate sleep and rest. Some nurses used different comfort measures they found to help comfort an infant to promote sleep and rest (Table 5). Some nurses used a Halo swaddle on the infant, which is a wearable blanket with Velcro that holds an infant's arms snug to the midline chest position. Most of these infants, because of their fussiness, will wiggle their arms and bodies loose from blankets when being swaddled. The halo swaddle helped prevent the infants from waking themselves because the arms are held secure in the swaddle. Also, a few of the nurses placed the infant's arms and hands midline with very tight swaddle with blanket or Halo swaddle, in order to mimic the tightness of the womb to promote sleep. Some nurses were found singing softly to the infants while holding them in order to promote sleep. Some nurses left the room quiet or with white noise from sound machine or white noise from TV while the infant was sleeping in the crib. A few of the nurses laid the infant's abdomen across the nurse's legs to comfort an infant's upset gastrointestinal tract. Also, some nurses used an activated heel 
warmer over one layer of clothing to the infant's abdomen to comfort an infant's upset gastrointestinal tract. Only a few nurses carried infants in Moby wraps or front carrying backpacks while the nurse walked in the hallways or performed charting on the infants. Some nurses would swaddle an infant and secure the infant in a stroller and walk around the unit with the infant. Many times a nurse would pass the infant in the stroller to a PCA to walk with the infant until the infant fell asleep.

All of the nurses stated that decreasing environmental stimuli (light, noise, touch) helped the infants to sleep and rest. Also, all of the nurses stated that the process of learning the baby allows the nurse to understand what kinds of comfort measures an infant needs to sleep or rest.

Culture of care and adequate rest and sleep (observational results). To further demonstrate the culture of care provided by NICU nurses to infants with NAS, all 12 of the interviewed nurses were observed for how the nurse provided adequate rest and sleep for an infant (see Table 5). Although there were similarities in this type of care, there were also differences.

With a fussy or awake infant, all of the nurses provided some kind of swaddling of the infant, although the way the infant was swaddled varied. Ten of the 12 nurses swaddled the infant with a blanket, while two of the nurses used a Halo swaddle. However, one of the nurses using the Halo swaddle placed the infant's arms and hands midline before the swaddle was secured with velcro and then placed the infant in a Moby wrap, while the other nurse using the Halo swaddle placed the arms in an "arms flexed, fist up" natural position of most newborns.

All but one of the infants was held to promote rest and sleep. The infant who was not being held was placed in the crib first and then the swing was awake and not fussy when observed. The other infant who was not fussy progressed from being held to the crib and then to 
the swing to remain sleeping. Seven of the held infants were simultaneously being rocked to promote sleep. Another of the held infants was bounced and the infant's bottom was patted to promote sleep. There were two interesting forms of holding an infant who had an gastrointestinal disturbances that was observed. One form of holding was with the infant lying prone over the legs of the nurse while sitting down. This infant’s abdomen to nurses’ legs position was observed throughout participant observation to provide relief from gastrointestinal disturbances, which is a symptom of withdrawal. Another comfort measure for gastrointestinal disturbances was the use of an activated heel-warmer over one layer of clothing on the infant's abdomen, which was used by Nurse 11 .

Most nurses were observed trying to place the infants in the crib if they would remain asleep. Nurses stated in participant observation that many families may not have a swing at home or financially able to afford the type of swing that is currently on NICU 3; therefore, nurses tried to place the infant in the crib first. If the infant could not remain asleep, the nurse would place the infant in the swing.

Six of the observed nurses used a pacifier to help comfort the infant to promote rest and sleep. Two of the nurses were observed singing softly to the infants. Seven of the nurses provided white noise in the room for the infant, while three of the nurses kept the room quiet. The nurse carrying the infant in the Halo swaddle and Moby wrap was observed in the hallway and another nurse was talking to the baby in the nurse's station. 
Table 5

Comparisons of Performed Rest and Sleep Measures by NICU 3 Nurses

\begin{tabular}{|c|c|c|c|c|c|}
\hline Nurse 1 & Nurse 2 & Nurse 3 & Nurse 4 & Nurse 5 & Nurse 6 \\
\hline $\begin{array}{l}\text { State of the } \\
\text { baby: } \\
\text { Fussy/crying }\end{array}$ & $\begin{array}{l}\text { State of the } \\
\text { baby: } \\
\text { Almost } \\
\text { asleep }\end{array}$ & $\begin{array}{l}\text { State of the } \\
\text { baby: } \\
\text { Awake, but } \\
\text { not fussy }\end{array}$ & $\begin{array}{l}\text { State of the } \\
\text { baby: } \\
\text { Fussy/crying }\end{array}$ & $\begin{array}{l}\text { State of the } \\
\text { baby: } \\
\text { Fussy/crying }\end{array}$ & $\begin{array}{l}\text { State of the } \\
\text { baby: } \\
\text { Fussy/crying }\end{array}$ \\
\hline $\begin{array}{l}\text { Swaddled } \\
\text { Holding } \\
\text { Rocking } \\
\text { Talking to } \\
\text { baby } \\
\text { Singing to } \\
\text { baby } \\
\text { Pacifier in } \\
\text { mouth } \\
\text { White noise } \\
\text { Machine } \\
\text { If calm, in crib }\end{array}$ & $\begin{array}{l}\text { Swaddled } \\
\text { Holding } \\
\text { Pacifier in } \\
\text { mouth } \\
\text { Crib first, } \\
\text { then } \\
\quad \text { Swing } \\
\text { Quiet }\end{array}$ & $\begin{array}{l}\text { Swaddled } \\
\text { Pacifier in } \\
\text { mouth } \\
\text { TV on for } \\
\text { white } \\
\text { noise } \\
\text { Swing (tried } \\
\text { crib } \\
\text { first) } \\
\text { Quiet }\end{array}$ & $\begin{array}{l}\text { Swaddled } \\
\text { Room cool } \\
\text { Holding } \\
\text { Rocking } \\
\text { Talking to } \\
\text { baby } \\
\text { Singing to } \\
\text { baby } \\
\text { Pacifier in } \\
\text { mouth } \\
\text { TV off } \\
\text { White noise } \\
\text { Machine } \\
\text { If calm, in crib }\end{array}$ & $\begin{array}{l}\text { Arms and } \\
\text { hands } \\
\text { midline with } \\
\text { very } \\
\text { tight } \\
\text { swaddle or } \\
\text { Halo } \\
\text { swaddle } \\
\text { Holding very } \\
\text { close } \\
\text { Carry infant in } \\
\text { Moby wrap } \\
\text { Infant } \\
\text { swaddled in a } \\
\text { stroller and } \\
\text { walked around }\end{array}$ & $\begin{array}{l}\text { Halo swaddle } \\
\text { Room cool } \\
\text { Holding very } \\
\text { close } \\
\text { Rocking in } \\
\text { rocking } \\
\quad \text { Chair } \\
\text { Pacifier in } \\
\text { mouth } \\
\text { White sound } \\
\quad \text { machine }\end{array}$ \\
\hline
\end{tabular}


Table 5 Continued

Comparisons of Performed Rest and Sleep Measures by NICU 3 Nurses

\begin{tabular}{|c|c|c|c|c|c|}
\hline Nurse 7 & Nurse 8 & Nurse 9 & Nurse 10 & Nurse 11 & Nurse 12 \\
\hline $\begin{array}{l}\text { State of the } \\
\text { baby: } \\
\text { Fussy/crying }\end{array}$ & $\begin{array}{l}\text { State of the } \\
\text { baby: } \\
\text { Fussy/crying }\end{array}$ & $\begin{array}{l}\text { State of the } \\
\text { baby: } \\
\text { Fussy/crying }\end{array}$ & $\begin{array}{l}\text { State of } \\
\text { the baby: } \\
\text { Fussy }\end{array}$ & $\begin{array}{l}\text { State of the } \\
\text { baby: } \\
\text { Fussy/crying }\end{array}$ & $\begin{array}{l}\text { State of the } \\
\text { baby: } \\
\text { Fussy/crying }\end{array}$ \\
\hline $\begin{array}{l}\text { Swaddled } \\
\text { Holding } \\
\text { Rocking } \\
\text { Talking to } \\
\text { baby } \\
\text { Pacifier in } \\
\text { mouth } \\
\text { White noise } \\
\text { from } \\
\text { sound } \\
\text { machine } \\
\text { Heel warmer } \\
\text { to } \\
\text { infant's } \\
\text { abdomen } \\
\text { If calm, in } \\
\text { crib } \\
\text { If not calm, } \\
\text { swino }\end{array}$ & $\begin{array}{l}\text { Swaddled } \\
\text { Holding in } \\
\text { breastfeeding } \\
\text { position } \\
\text { Rocking } \\
\text { Talking to } \\
\text { baby } \\
\text { White noise } \\
\text { from } \\
\text { sound } \\
\text { machine } \\
\text { If calm, in } \\
\text { crib }\end{array}$ & $\begin{array}{l}\text { Swaddled } \\
\text { Holding } \\
\text { infant on } \\
\text { shoulder } \\
\text { Rocking } \\
\text { Talking to } \\
\text { baby } \\
\text { In nurse's } \\
\text { station }\end{array}$ & $\begin{array}{l}\text { Swaddled } \\
\text { Baby } \\
\text { lying } \\
\text { across her } \\
\text { legs with } \\
\text { infant in } \\
\text { prone } \\
\text { position, } \\
\text { to comfort } \\
\text { baby's } \\
\text { stomach. } \\
\text { Talking to } \\
\text { baby } \\
\text { White } \\
\text { noise } \\
\text { from } \\
\quad \text { TV } \\
\text { If calm, } \\
\text { swino }\end{array}$ & $\begin{array}{l}\text { Swaddled } \\
\text { Holding } \\
\text { Rocking } \\
\text { Talking to } \\
\text { baby } \\
\text { Pacifier in } \\
\text { mouth } \\
\text { Quiet room } \\
\text { Heel warmer } \\
\text { to } \\
\text { infant's } \\
\text { abdomen } \\
\text { If calm, in } \\
\text { crib } \\
\text { If not calm, } \\
\text { swing }\end{array}$ & $\begin{array}{l}\text { Swaddled } \\
\text { Holding } \\
\text { Bouncing } \\
\text { Patting } \\
\text { bottom } \\
\text { Talking to } \\
\text { Pacifier in } \\
\text { mouth } \\
\text { White noise } \\
\text { from sound } \\
\text { machine } \\
\text { If calm, in } \\
\text { crib }\end{array}$ \\
\hline
\end{tabular}

Nurses use many similar comfort measures to help the infant to sleep and/or rest and include swaddling, holding, rocking, talking to the infant, and a pacifier in mouth. Most nurses found that if the infant was calm, the infant could be laid in the crib. If the infant was not calm and other comfort measures were tried, the infant could be put in the swing.

Feeding. Although much of the literature surrounding NAS and infant feeding concerns the effects of breastfeeding and withdrawal symptoms, this research did not provide examples of breast feeding mothers. Most of the infants on NICU 3 were bottle-fed formula by a nurse or PCA. Throughout individual interviews nurses described feeding an infant with NAS as 
“difficult” and "different with each infant.” Most of the nurses stated that infants had a “disorganized suck”, had trouble latching, and “required chin and cheek” support while feeding. Also, many of the nurses indicated that the infants saw "food as comfort" while at the same time "food is not comfort." The nurses described the act of sucking on the nipple of the bottle and receiving formula as a comfort to the infants. However, in regards to "food is not comfort" many infants would over-eat because of excessive sucking for comfort and possibly vomit part or all of the feeding. Participant 06 describes an infant's excessive suck and the potential for vomiting.

A lot of these kids have an excess suck especially at the beginning. They will suck on anything. They'll suck on your finger, they'll suck on the pacie, they'll suck the fire out of the pacifier but they'll suck on a bottle whether or not they're hungry. Well all of the sudden, they're so full, you pick 'em up to burp but and everything comes back out that's fine until you have all your phenbarb, your morphine, your clonidine, your okay so then they’ve just thrown all that up because you can tell cause there's red everywhere, you don't know how much has come out so a lot of times we don't re-dose them, we just wait 'til next time so that can make for three very lousy hours.

In addition, many nurses stated in interviews that when an infant would eat and possibly feel comfort at first from becoming full, the formula could possibly further irritate an already sensitive gastrointestinal tract from the withdrawal from maternal drugs.

Culture of care and feeding (observational results). To further demonstrate the culture of care provided by NICU nurses to infants with NAS, all 12 of the interviewed nurses were observed while formula feeding an infant with NAS. The descriptions of these feedings are recorded in the (see Table 6) below. Most nurses started with a standard approach to bottle- 
feeding, which was learned and practiced in their previous NICU experiences. These standard nursing practices are swaddle the baby, infant held close, eye contact with the baby, talking to the baby, and burping half way through the feeding and at the end of the feeding. However, as the nurse learned the baby, many differences in feeding methods were observed. Each nurse performed many of the standard feeding practices with adaptations to fit the baby's needs for effective feeding. The differences in feeding practices included four nurses with the infant unswaddled, five nurses holding the infant away from the nurse's body, and one nurse using the Halo swaddle to feed the infant. One of the four nurses who unswaddled the infant during feeding placed mittens on the infants hands to prevent the infant from scratching his face during the feeding. Seven nurses provided chin and cheek support to the infant while feeding. One nurse used a cool cloth to wipe the infant's face to keep the infant more awake during the feeding.

In regards to burping, six of the nurses frequently burped the infant (more often than half way through the feeding and at the end). Although this was observed in participant observation, this frequent burping was not mentioned during the interviews. Nurse 10 explained the effects of formula on some infants, “sometimes food isn’t always a comfort measure to them and especially if the formula is hurting their bellies because they're already cramping, they're already having diarrhea a lot of times." However, one of the nurses did not burp the infant until the end of the feeding because she found that the infant fed better without a break for burping. This seemed to be the exception but was what worked for this one infant in regards to burping. Eight of the infants took from 15 to 25 minutes to feed, while four of the infants took the full 30 minutes. At 30 minutes, most nurses know that they are to stop the feeding in order for the infant to resume sleep and rest. 
Table 6

Field Note Observations for Infant Feeding: Nurses 1-6

\begin{tabular}{|c|c|c|c|c|c|}
\hline Nurse 1 & Nurse 2 & Nurse 3 & Nurse 4 & Nurse 5 & Nurse 6 \\
\hline $\begin{array}{l}\text { State of the } \\
\text { baby: calm }\end{array}$ & $\begin{array}{l}\text { State of the } \\
\text { baby: calm }\end{array}$ & $\begin{array}{l}\text { State of the } \\
\text { baby: } \\
\text { Calm and } \\
\text { sleepy }\end{array}$ & $\begin{array}{l}\text { State of the } \\
\text { baby: } \\
\text { calm }\end{array}$ & $\begin{array}{l}\text { State of the } \\
\text { baby: } \\
\text { fussy }\end{array}$ & $\begin{array}{l}\text { State of the } \\
\text { baby: calm }\end{array}$ \\
\hline $\begin{array}{l}\text { Baby } \\
\text { unswaddled } \\
\text { Baby held } \\
\text { close } \\
\text { Mittens on } \\
\text { baby } \\
\text { Looking at } \\
\text { baby } \\
\text { Talking to } \\
\text { baby } \\
\text { Feeding for } \\
20 \\
\quad \text { minutes } \\
\text { Burping } 1 \text { ² } \\
\text { way } \\
\text { and at end }\end{array}$ & $\begin{array}{l}\text { Baby } \\
\text { swaddled } \\
\text { Baby held } \\
\text { close } \\
\text { Looking at } \\
\text { baby } \\
\text { Talking to } \\
\text { baby } \\
\text { Feeding } \\
\text { infant for } \\
\quad 15 \text { minutes } \\
\text { Burping } 1 / 2 \\
\text { way } \\
\text { and at end }\end{array}$ & $\begin{array}{l}\text { Baby } \\
\text { unswaddled } \\
\text { Baby held } \\
\text { close } \\
\text { Looking at } \\
\text { baby } \\
\text { Talking to } \\
\text { baby } \\
\text { Cool cloth to } \\
\text { face } \\
\text { to keep } \\
\text { infant } \\
\text { awake } \\
\text { Chin and } \\
\text { cheek } \\
\text { support } \\
\text { Slow feeding } \\
\text { (30 } \\
\text { minutes) } \\
\text { Frequent } \\
\text { burping } \\
\text { to keep } \\
\text { infant } \\
\text { awake }\end{array}$ & $\begin{array}{l}\text { Baby } \\
\text { swaddled } \\
\text { Baby held } \\
\text { away } \\
\text { Looking at } \\
\text { baby } \\
\text { Talking to } \\
\text { baby } \\
\text { Chin and } \\
\text { cheek } \\
\text { support } \\
\text { Feeding for } \\
20 \\
\text { minutes } \\
\text { Burping } 1 / 2 \\
\text { way } \\
\text { and at end }\end{array}$ & $\begin{array}{l}\text { Baby } \\
\text { swaddled } \\
\text { Baby held } \\
\text { close } \\
\text { Looking at } \\
\text { baby } \\
\text { Talking to } \\
\text { baby } \\
\text { Chin and } \\
\text { cheek support } \\
\text { Feeding for } \\
25 \text { minutes } \\
\text { Burping } \\
\text { frequently } \\
\text { because baby } \\
\text { crying }\end{array}$ & $\begin{array}{l}\text { Halo swaddle } \\
\text { Baby held } \\
\text { away } \\
\text { Looking at } \\
\text { baby } \\
\text { Talking to } \\
\text { baby } \\
\text { Feeding for } \\
20 \\
\text { minutes } \\
\text { Frequent } \\
\text { burping } \\
\text { Held up on } \\
\text { shoulder to } \\
\text { help } \\
\text { with } \\
\text { stomach } \\
\text { pain }\end{array}$ \\
\hline $\begin{array}{l}\text { Differences: } \\
\text { Baby } \\
\text { unswaddled } \\
\text { Mittens on } \\
\text { baby to } \\
\text { prevent } \\
\text { scratching } \\
\text { face } \\
\text { with hands. }\end{array}$ & $\begin{array}{l}\text { Differences: } \\
\text { none }\end{array}$ & $\begin{array}{l}\text { Differences: } \\
\text { Baby } \\
\text { unswaddled } \\
\text { Cool cloth to } \\
\text { face } \\
\text { Chin and } \\
\text { cheek } \\
\text { Support } \\
\text { Full } 30 \\
\text { minutes to } \\
\text { feed } \\
\text { Frequent } \\
\text { burping }\end{array}$ & $\begin{array}{l}\text { Differences: } \\
\text { Baby held } \\
\text { away } \\
\text { Chin and } \\
\text { cheek } \\
\text { support }\end{array}$ & $\begin{array}{l}\text { Differences: } \\
\text { Chin and } \\
\text { cheek } \\
\text { support } \\
\text { Frequently } \\
\text { burping }\end{array}$ & $\begin{array}{l}\text { Differences: } \\
\text { Halo swaddle } \\
\text { Baby held } \\
\text { away } \\
\text { Frequent } \\
\text { burping } \\
\text { Held up on } \\
\quad \text { shoulder }\end{array}$ \\
\hline
\end{tabular}


Table 6 Continued

Field Note Observations for Infant Feeding: Nurses 7-12

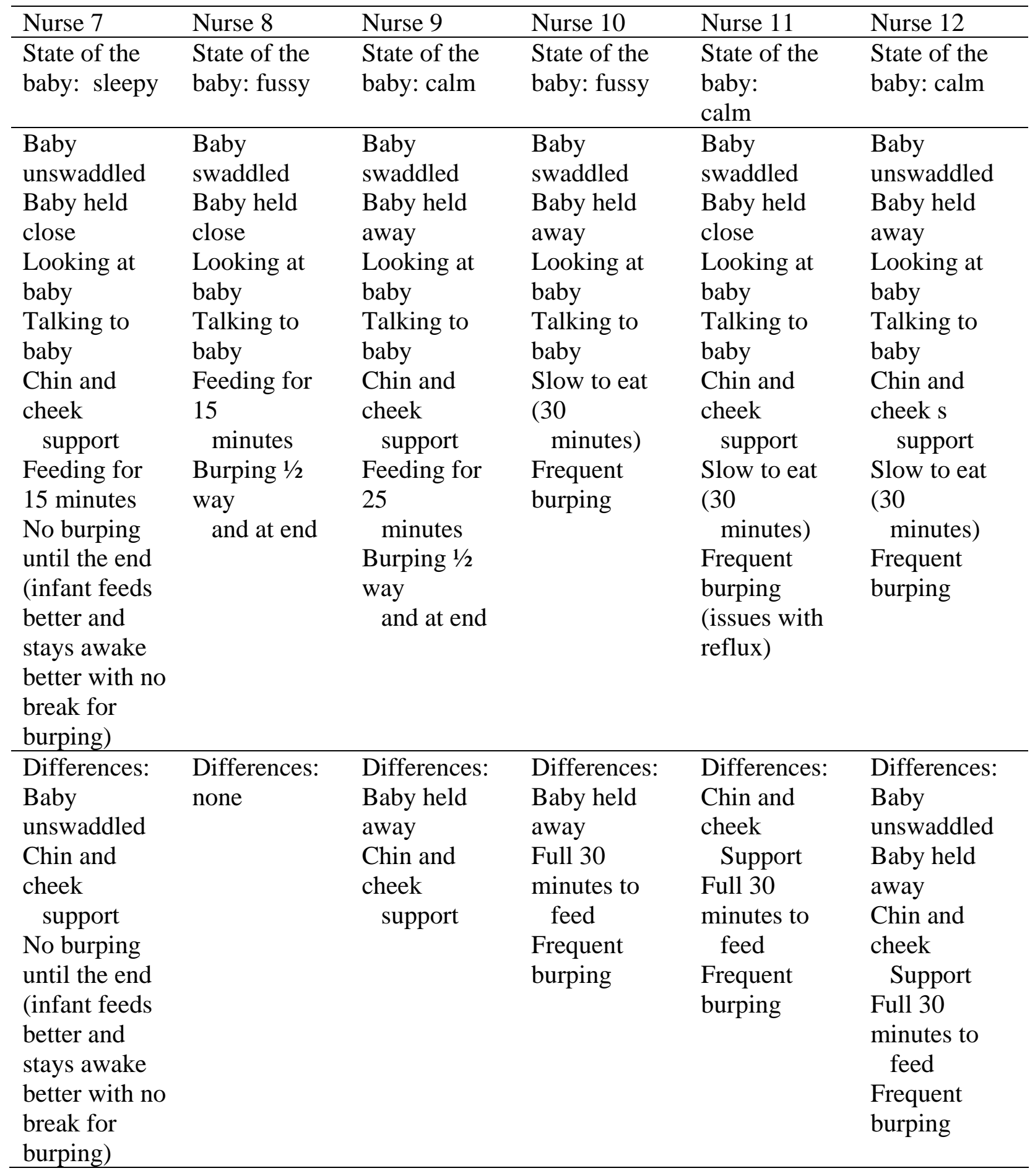


The theme of, learn the baby, with the subthemes of routine care, comfort care, environment, adequate rest and sleep, and feeding were all observed during participant observation and described during individual interviews.

\section{Theme 2: Core Team Relationships}

Only full-time "core” team nurses were observed and interviewed for this ethnography. The researcher felt like these nurses would offer a richer and deeper understanding of the culture of care provided to infants with NAS. In the beginning stages of participant observation, it became clear that besides a culture of care towards infants, there was also a culture of care among the core team nurses. From the observational data and the interview data two subthemes emerged that support this positive interaction among core team nurses; support and interpersonal relationships.

Support. From individual interviews, this small group of core team nurses all stated that they felt very supported at work by the other core team nurses, PCAs, cuddlers, hospital security, nursing administration, and by the nurse practitioners. Although there were many levels of support, the interviewed nurses all stated that the support of the core team made "all the difference.”

We have a very strong cohesive core group on night shift and we understand each other. We think along the same lines, we think 'what is best for the baby?' not 'what is best for the paperwork?' Without that support system and my coworkers, it would be difficult to work there.

The core team nurses stated in individual interviews that the core team supported each other by sharing the "same passion" for the babies, "shared knowledge" of care for infants and families, "emotional support”, "shared language”, "shared strategies for care”, "shared enjoyment” in 
caring for these infants, and "shared understanding" of the difficulties in caring for infants with NAS. All of this shared understanding of the work was summed up in the frequently used phrase, "you get it.”

We're able to understand each other more, we're able to understand the babies more because we’ve worked with them you know just kind of over-consistent so something that might need further explanation to a nurse that's not done this for a while, you know you can give a report to one of the nurses on the core team and it's just kind of, you just kind of understand each other. You get it.

Field notes and individual interviews revealed many instances where the nurses experienced teamwork on the unit in regards to the care of the infants. Many times I observed one nurse helping another nurse with the care of her baby. The nurses stated that there were "not enough hands.” Therefore, the nurses also help each other with their infants’ care when needed.

Participant 11 described this teamwork in detail.

The teamwork is just unbelievable and I mean you don't hesitate to ask anybody anything. I've worked places before where there's some people that you don't ask to do anything but there's not one single person that I work with that you can't ask and I'm honest, I mean anybody would do anything to help you.

When asked the question "what makes work easier for you?” the response most stated in interviews was "working with the core team.” When asked the question, "how do you cope with the difficult days?”, core team nurses had the similar response of "working with the core team.” Participant 07 speaks to "getting used to" and the improvement of coping mechanisms over time among nurses. 
I actually think you just learn your coping mechanisms. Your coping mechanisms improve because at first, you know coming into this, it was, I guess more stressful because the noise level maybe sometimes isn't quite what you're used to and so you learn in that timeline you know everything's gotta be done, but then you learn that 'yes, this needs to be done but this baby is really having a hard time," so you kind of learn to prioritize and you take what's most important and deal with it.

Many nurses described periods of joking, laughing, and teasing amongst themselves to cope and relieve tensions during interviews. I also observed these behaviors of teasing and laughing between the core team nurses during participant observation. I observed many nurses talking with each other about a specific baby and its care, discussions on what to try next and concerns for the infant. Also in regards to coping and how nurses support each other, Participant 09 states, "you just get the babies through as well as you can and you just focus on what you can do." Nurses stated this phrase, "focusing on what you can do," often during participant observation and in interviews.

Interpersonal relationships. Along with support from core team relationships, I also observed nurses exhibiting behaviors of friendship. Participant 08 states, "I think on night shift, we're particularly lucky because for the most part, we get along extraordinarily well. We have a crew that genuinely enjoys each other." One nurse described the nurses as "a close knit group of girls.” Three of the interviewed nurses described their relationships with other core team nurses as "like a family." Four of the nurses mentioned that the core team of nurses was a "smaller number of co-workers" and it was therefore easier to form closer relationships.

Also, an interesting quote, "we are bunch of older nurses," was stated in many of the interviews. The core team nurses in their 50s and 60s all stated in interviews that they had been 
NICU nurses for many years and did not miss performing all of the "highly technical skills" they used in NICU 1. Also, the nurses stated that they "did not fear losing proficiency in these NICU skills” because they worked in an area of the NICU that does not require these same skills. Also, during participant observation many nurses were observed for caring behaviors such as hugging each other, joking, and teasing with each other, sharing personal information about self and families, showing pictures of family members to each other, sharing future plans such as vacations and schooling, and conversations about getting together to eat a meal together. Table 7 below shows some of the observations of nurses exhibiting interpersonal connections during the workday. Also included in this table are examples of how core team nurses were working together and supporting each other in the care of infant. 
Table 7

Field Note Observations of Interpersonal Connections of 12 Nurses

Observation

Nurse 12 (while gathering linens for the room) asks Nurse 4 about her vacation.

Nurses 10 sitting in the nurse's station collaborating about an infant's feeding difficulties with Nurse 2.

Nurse 7 asking other nurses if they want anything (food or drink) from the cafeteria.

Night shift Nurse 8 hugging Nurse 6 when they saw each other in the nurse's station.

Nurse 11 asking Nurse 2 for help with her baby that is fussy so that she can care for another baby.

Nurse 5 asking if she can take the baby in the stroller for a walk around the unit while Nurse 1 does her charting.

Nurse 9 talking with Nurse 2 about her summer class and the final paper.

During report, nurses discussing the infant's Finnegan scores and sharing strategies for comfort that have worked.

Nurse 4 offering to bathe another nurse's baby.

PCA offering to feed infant.

Nurse 6 discussing infant's high score with Nurse 3 and decisions about calling the doctor.

Nurse 9 standing close to Nurse 10 and talking quietly about personal life issues.

Nurse 10 saying to Nurse 9, "let me know what you find out from social work about the baby."

Nurse 1 sharing "what works" with this baby for feeding with a PCA.

Nurse 4 checking meds with another nurse and asking how her "pod" is doing tonight.

Nurse 6 helping Nurse 5 to strap on the front carrying backpack to hold the baby.

Nurses talking and laughing in the break room over a meal.

Nurses greeting each other when they come in the door of the unit.

Nurses waiting on each other to walk off the unit at the end of the shift.

Nurse 10 passing a baby to another nurse and saying "I could not do this without you."

Nurses sitting and standing at the nurse's station laughing and joking.

Nurse 8 asking Nurse 3 if she needs help with her infants.
Coding

Personal interactions

Sharing personal lives

Genuine care for each

Other

Laughing/joking/teasing

Collaboration of care Strategies for care

What works

Shared language

Troubleshooting

Shared concern for

Babies

Teamwork

Support

Physical help

Emotional support 
Observation

Nurse changing the bed for a nurse while she is giving an infant a bath.

Nurses sharing candy.

Nurse 9 at Omnicell with Nurse 11 helping to figure out a medication discrepancy.

Nurse 3 showing Nurse 8 and Nurse 12 pictures of her grandchild.

During change-of-shift report, Nurse 11sitting next to Nurse 8 and reporting details of her 3 infants with statements such as, "You know how he cries," "his excoriation on his face is getting better," "the heal warmer to his belly helped him to sleep" and "he is still tremorring quite a bit."

A secretary holding an infant while she answers the phone.

Nurse 8 talking with Nurse 3 and Nurse 4 about a time they went out to dinner in the past week.

Nurse 4 going to check on the crying that she is hearing down the hall.

Nurse 6 handing a baby to a cuddler and showing him how the baby likes to be bounced and patted on the bottom.

Nurse 2 teases Nurse 7 about dropping so many supplies today.

Nurse 3 patting the back of Nurse 12 and saying, "We just have to pray that he will be okay with his grandma.” (in reference to a baby being discharged to the grandmother's home)
Teamwork

Interpersonal relationships

Interpersonal relationships 
Participant 07 summed up the feelings of core team nurses regarding support and interpersonal relationships with these statements.

I love coming to work and being with the coworkers that I'm with and we solve problems together. We never have one nurse stuck out in left field trying to figure out what to do with her baby and she doesn't feel, like she can really, you know, depend on anybody or ask anybody what to do. If something goes wrong, we solve it as a group. If something is good, we celebrate is as a group. I know that those girls have my back just like I've got theirs.

The theme of core team relationships with the subthemes of support and interpersonal relationships were all observed during participant observation and described during individual interviews.

\section{Theme 3: Role Satisfaction}

From participant observation and individual interviews, the nurse's role satisfaction involved the subthemes of nurturer or comforter and becoming an expert. Each subtheme further described how the nurse's role was meaningful and satisfying.

Nurturer or Comforter. The literature has limited research of the nurse's role in relation to caring for an infant with NAS (Fraser et al., 2007; Murphy-Oikonen et al., 2010). During the individual interviews with core team nurses, the open-ended question was asked, "What role do you see yourself in with these infants?” The interviewed nurses gave the responses of nurturer, one who genuinely loves the family, like a long lost family member, protector, advocate, their support system, caretaker, nurse, medical support, the one who gives them a good start, educator, in the middle, fire fighter, social worker, police, director of a day care, and surrogate mommy (but not take the place of their mommy). These varied responses were coded into the category of 
"comforter or nurturer" and "multiple hats" and then condensed again into the subtheme of nurturer or comforter. It seemed that every role, including the category of "multiple hats," had an element of comforting the infant or nurturing the infant, therefore; both could fall into the theme of nurture or comforter for the baby. In describing the type of care provided by a nurse to an infant, one nurse stated,

I feel like it’s the same care I provided my own baby, my own child. It's a very intense, it's a very nurturing care again because so many times, they don't have anyone but us and so they don't know the feeling of the security, they don't know the feeling of touch and love and that gentle sense and skin to skin and the you know, they just, they don't have that sometimes.

Other nurses stated that they felt like the kind of care they provided was "just like being a mommy to them (but not their mommy).” One nurse in particular described her role as a long lost family member, “you know the one that, the family member that doesn’t ever hang out with you or have fun but the one that you call whenever you have a problem, that's how I kind of feel.” This nurse also stated that she cares not only for the infant but the family as well and “doesn’t feel like a perfect stranger” to them. This connectedness to the infant as well as the family was particularly evident in this nurse's interview.

The subtheme of nurturin or comforting an infant with NAS was described by the core team nurses' responses to this question, "What is the type of care that you provide to these infants?” The nurses gave these descriptions of care: enjoyable care, hands on care, physical care, emotional care, intense care, and developmental care. Core team nurses described the various kinds of care they provided. Participant 01 described enjoyable care as,

Taking your time with them because you can’t just go in and hurry. I enjoy talking to 
'em and doing things and just you know if you get done with the feeding you don’t just immediately put 'em down in the bed. You take some time to hold 'em or do whatever. Another nurse described the care that she provides to infants with NAS as emotional care.

I think there's a lot of what I would call emotional care in the, it's the holding them till they're calm. Um a lot of people might say that we just use the time to sit around and do nothing, but the fact is what you're doing is you're holding them until they are relaxed because otherwise, they may not sleep in between feedings. You wrap their arms up and you hold your hands on them or you hold your hands on their legs until you can feel them melt under your hands and they a lot of times then they'll go to sleep, so there's the physical care and then what I call the emotional care or just holding them until they melt. Participant 06 describes the kind of care she provided as hands-on caring that is one-on-one and also addresses the developmental needs of the infant.

I personally try to spend as much individual time with that baby as possible getting 'em together so there's a lot of hands on cause we do their vital signs, but there's a lot of comforting, a lot of comfort measures, learning what the baby likes, bathing, swaddling, so on and so forth. Music, do they like music? Do they like white noise? Do they not like white noise? A lot of interaction as far as developmentally cause you see these babies sometimes for a couple months and so you're with them and you need to learn you know as they get older, you got to help them developmentally, play with toys, make 'em laugh, you know just like they were your own.

Lastly, many nurses described the kind of care they gave to infants with NAS as "intense" because an infant will be doing well (sleeping) for a few hours and then the infant will become fussy, irritable, and in pain for many hours without being able to be consoled. All observed and 
interviewed nurses stated that providing nurturing or comforting to these infants was their role.

Becoming an expert. Because NICU 3 is the only known nursing unit of its kind, this question was asked during the individual interviews, "What is it like working with a specialize team of nurses and other professionals?” The answer stated by many of the nurses was that becoming an expert benefited the baby. The categories that comprise the subtheme of becoming an expert were benefit to the baby, experts score appropriately, experts anticipate the baby’s needs, trial and error, primary care nursing, consistency of care, learning the baby, commitment for the staff to keep learning, family centered care, what the baby needs, and what the family needs. All of these categories encompass what it takes for a nurse to become an expert. Participant 05 described the specialization of care that is given to these infants.

When you specialize in something, you become an expert on that certain thing so just like, just like you know a child everyday, you know they become an expert on how to do their own hair, you know they may not let their mom do their hair because they know exactly how to do it. I believe that when you do something everyday, you become an expert and you become really good and then that benefits the patient. When I know the baby I am an expert with that baby.

From participant observation and individual interviews, the nurse’s role satisfaction involved the subthemes of nurturer or comforter and becoming an expert to further describe how the nurse's role was meaningful and satisfying. Both of these subthemes of role satisfaction describe the culture of care provided by NICU nurses to infants with NAS.

Culture of care and the role of nurturer or comforter (observational results). To further demonstrate the culture of care provided by NICU nurses to infants with NAS, all 12 of the interviewed nurses were observed during an initial encounter with the infant at the beginning of 
their shift (Table 8). As a general observation it was clear that the nurses cared deeply for the infants by the descriptions presented in the chart. Many of these nurses display similar actions in encountering the infant, but there are also some differences.

All of the nurses looked at the baby and either said the infant's name or a term of endearment such as "sweetheart" or "sweet boy." Five of the nurses continued to talk to the infant while providing care. Six nurses reswaddled the fussy infants who had become unswaddled or loose in their blankets. One of these six nurses swaddled the infant in the Halo swaddle. The nurse who used the Halo swaddle was a nurse who was observed multiple times using this swaddle and felt like the Velcro on the swaddle helped to secure the swaddle more than a blanket. Five of the nurses put a pacifier in a fussy infant's mouth to help comfort them. If an infant was asleep or awake and not fussy, a pacifier was not used to provide comfort during these observations.

The variations for touching the infants were diverse and possibly connected to the nurse's knowledge of the baby. Gentle touch by Nurse 1 was used with an infant who needed to be woken to assess and feed. Another nurse (Nurse 4), while the infant was awake, put her hand on the infant's abdomen and kept it there while she talked with the infant. Nurse 5, who placed the infant in the Halo swaddle, placed the infant in the breastfeeding position, which is the infant's abdomen to the nurse's abdomen. This nurses stated that this position, like the previous nurse's hand on the abdomen, provided "a feeling to the infant that someone is touching them. " Nurse 7 touched the infant only when needed. Before the infant was awake, the nurse gently performed vital signs and an assessment. When Nurse 7 tried to get an infant to smile, and when the infant did smile, the nurse stated, "I knew that smile was in there." Two nurses used touch in the form of patting the infant's bottom. One of the occurrences of patting the bottom occurred with the 
infant lying across the nurse's knees. Nurse 12 used patting the bottom with a swaying motion, while Nurse 10 used swaying only to comfort a fussy infant.

Nurse 8 was observed entering the infant's room, greeting the baby and talking with the mother about the timing of the infant's next feeding. The nurse did not take the baby from the mother but allowed her to care for her infant without interruption. Nurse 11 was observed trying to keep an infant calm in order for the infant to fall asleep before the next feeding; therefore, she reswaddled the infant and passed the infant to a PCA who could hold and comfort the infant until it was time for the next feeding. Nurse 4 and Nurse 12 were observed while singing to their infants. 
Table 8

Field Note Observations for Initial Encounter with the Baby: Nurses 1-6

\begin{tabular}{|c|c|c|c|c|c|}
\hline Nurse 1 & Nurse 2 & Nurse 3 & Nurse 4 & Nurse 5 & Nurse 6 \\
\hline $\begin{array}{l}\text { State of the baby: } \\
\text { Asleep }\end{array}$ & $\begin{array}{l}\text { State of the baby: } \\
\text { Fussy/crying }\end{array}$ & $\begin{array}{l}\text { State of the baby: } \\
\text { Fussy/crying }\end{array}$ & $\begin{array}{l}\text { State of the baby: } \\
\text { Awake }\end{array}$ & $\begin{array}{l}\text { State of the baby: } \\
\text { Fussy/crying }\end{array}$ & $\begin{array}{l}\text { State of the baby: } \\
\text { Fussy/crying }\end{array}$ \\
\hline $\begin{array}{l}\text { Walk quietly in the } \\
\text { room to see the } \\
\text { baby in the crib. } \\
\text { During routine care, } \\
\text { infant wakes up. } \\
\text { Nurse looks at baby } \\
\text { and greets baby } \\
\text { with "good evening } \\
\end{array}$ & $\begin{array}{l}\text { Walks into the } \\
\text { room and picks the } \\
\text { baby up out of the } \\
\text { swing. Says the } \\
\text { baby's name and } \\
\text { takes the baby to } \\
\text { the crib to check the } \\
\text { diaper and re- } \\
\text { swaddled the infant. }\end{array}$ & $\begin{array}{l}\text { Walks into the } \\
\text { room and sees that a } \\
\text { cuddler is holding } \\
\text { the baby in a } \\
\text { rocking chair. } \\
\text { Nurse says the } \\
\text { infant's name out } \\
\text { loud and the infant } \\
\text { turns her head to the }\end{array}$ & $\begin{array}{l}\text { Walks into the } \\
\text { room and sees the } \\
\text { baby in the crib } \\
\text { awake with the } \\
\text { aquarium music } \\
\text { box playing. Nurse } \\
\text { sings to the baby, } \\
\text { "Are you ready for } \\
\text { a bath tonight }\end{array}$ & $\begin{array}{l}\text { Walks into the } \\
\text { room and says the } \\
\text { baby's name and } \\
\text { "how did you get } \\
\text { out of that } \\
\text { blanket?" Picks up } \\
\text { infant and holds the } \\
\text { infant close. While } \\
\text { still talking to the }\end{array}$ & $\begin{array}{l}\text { Walks up to the } \\
\text { PCA who is holding } \\
\text { and patting the baby } \\
\text { and says the } \\
\text { infant's name and } \\
\text { whispers “Are we } \\
\text { going to have a } \\
\text { better night tonight } \\
\text { sweetheart?" The }\end{array}$ \\
\hline $\begin{array}{l}\text { Continues to talk } \\
\text { and look at baby } \\
\text { with gentle } \\
\text { touching during the } \\
\text { assessment. }\end{array}$ & $\begin{array}{l}\text { Pacifier put in the } \\
\text { baby's mouth while } \\
\text { the nurse is looking } \\
\text { at and talking } \\
\text { quietly to the baby. } \\
\text { Baby picked up and } \\
\text { held close up on } \\
\text { nurse's shoulder. } \\
\text { Nurse disconnects } \\
\text { the baby from the } \\
\text { monitor and walks } \\
\text { out to the nurse's } \\
\text { station with the } \\
\text { baby. Infant still } \\
\text { fussy. }\end{array}$ & $\begin{array}{l}\text { nurse. Nurse comes } \\
\text { closer to the infant } \\
\text { and says, “Are you } \\
\text { have a tough time } \\
\text { tonight?" Nurse } \\
\text { continues to talk } \\
\text { softly to the baby as } \\
\text { the cuddler holds } \\
\text { the baby. The } \\
\text { pacifier is put in the } \\
\text { infant's mouth and } \\
\text { the infant is calmed } \\
\text { down while looking } \\
\text { at the nurse. }\end{array}$ & $\begin{array}{l}\text { ?’ Nurse } \\
\text { looks at the baby } \\
\text { and puts her hand } \\
\text { on the infant's } \\
\text { abdomen and holds } \\
\text { it there while she } \\
\text { continues to talk } \\
\text { with the infant. }\end{array}$ & $\begin{array}{l}\text { infant, places the } \\
\text { infant in a Halo } \\
\text { swaddle with arms } \\
\text { firmly placed } \\
\text { midline on the } \\
\text { abdomen and the } \\
\text { Velcro on the } \\
\text { swaddle attached } \\
\text { firmly. Picks up the } \\
\text { infant, holds infant } \\
\text { in the breastfeeding } \\
\text { position and carries } \\
\text { the baby to the } \\
\text { medication cart } \\
\text { outside of the room } \\
\text { so that she can see } \\
\text { her paperwork. }\end{array}$ & $\begin{array}{l}\text { nurse takes the } \\
\text { baby, is looking at } \\
\text { the baby, talking } \\
\text { and walking the } \\
\text { baby back to the } \\
\text { room. The infant is } \\
\text { re-swaddled and } \\
\text { starts to quiet down } \\
\text { while the nurse is } \\
\text { still talking to the } \\
\text { baby. The pacifier } \\
\text { is put in the infant's } \\
\text { mouth. The nurse } \\
\text { puts the baby in the } \\
\text { swing and puts on } \\
\text { the white noise } \\
\text { machine. Infant is } \\
\text { awake but not } \\
\text { crying. }\end{array}$ \\
\hline
\end{tabular}


Field Note Observations for Initial Encounter with the Baby: Nurses 7-12

\begin{tabular}{|c|c|c|c|c|c|}
\hline Nurse 7 & Nurse 8 & Nurse 9 & Nurse 10 & Nurse 11 & Nurse 12 \\
\hline $\begin{array}{l}\text { State of the baby: } \\
\text { Asleep }\end{array}$ & $\begin{array}{l}\text { State of the baby: } \\
\text { Awake and quiet }\end{array}$ & $\begin{array}{l}\text { State of the baby: } \\
\text { Fussy/crying }\end{array}$ & $\begin{array}{l}\text { State of the baby: } \\
\text { Very fussy }\end{array}$ & $\begin{array}{l}\text { State of the baby: } \\
\text { Fussy/crying }\end{array}$ & $\begin{array}{l}\text { State of the baby: } \\
\text { Fussy/crying }\end{array}$ \\
\hline $\begin{array}{l}\text { Nurse walks quietly } \\
\text { into the room to } \\
\text { check on the baby. } \\
\text { Infant is swaddled, } \\
\text { asleep with the } \\
\text { white noise } \\
\text { machine playing } \\
\text { from the swing. } \\
\text { When it is time for } \\
\text { the infant to feed, } \\
\text { the nurse quickly } \\
\text { gets a blood } \\
\text { pressure, heart rate, } \\
\text { respiratory rate and } \\
\text { temperature while } \\
\text { the infant is waking } \\
\text { up. As the infant is } \\
\text { waking, the nurse is } \\
\text { saying the baby's } \\
\text { name, looking } \\
\text { closely at the baby } \\
\text { to make eye contact } \\
\text { and touching the } \\
\text { baby only as needed } \\
\text { for the assessment. } \\
\text { The nurse is trying }\end{array}$ & $\begin{array}{l}\text { Nurse walks into } \\
\text { the room to see the } \\
\text { baby being held by } \\
\text { the mother. The } \\
\text { nurse leans over to } \\
\text { talk to the baby, } \\
\text { look at the baby and } \\
\text { comment on how } \\
\text { big he is getting. } \\
\text { The nurse lets the } \\
\text { mother know that } \\
\text { she will be coming } \\
\text { in to assess the } \\
\text { baby in } 30 \text { minutes } \\
\text { and that she will } \\
\text { bring in the formula } \\
\text { for the mom to feed } \\
\text { to the baby. }\end{array}$ & $\begin{array}{l}\text { Nurse walks into } \\
\text { the room to see a } \\
\text { cuddler rocking and } \\
\text { patting the baby. } \\
\text { The nurse comes up } \\
\text { to the baby, says the } \\
\text { baby's name and } \\
\text { looks at the baby. } \\
\text { The nurse says, "I } \\
\text { am so sorry that } \\
\text { your belly hurts. } \\
\text { Hopefully after } \\
\text { your bath, you will } \\
\text { feel better." The } \\
\text { nurse lets the } \\
\text { cuddler know that } \\
\text { sometimes the baby } \\
\text { likes to be laid with } \\
\text { its abdomen across } \\
\text { the cuddler's legs } \\
\text { and helps that infant } \\
\text { into this position. } \\
\text { The infant is still } \\
\text { crying at first, but } \\
\text { starts to calm down } \\
\text { as the infant's }\end{array}$ & $\begin{array}{l}\text { Nurse walks into } \\
\text { the room to see the } \\
\text { baby unswaddled, } \\
\text { crying and frantic in } \\
\text { the crib. The nurse } \\
\text { says the baby's } \\
\text { name and picks up } \\
\text { the baby and says, } \\
\text { "hey, hey, hey, it } \\
\text { will be alright. I've } \\
\text { got you." The } \\
\text { infant calms down a } \\
\text { little, but is still } \\
\text { crying. The nurse } \\
\text { holds the infant } \\
\text { close, sways back } \\
\text { and forth while } \\
\text { standing and talks } \\
\text { to the baby in order } \\
\text { to calm the infant. } \\
\text { The nurse asks } \\
\text { "where is your } \\
\text { pacie?" The nurse } \\
\text { finds the pacifier in } \\
\text { the blanket and puts } \\
\text { it in the infant's }\end{array}$ & $\begin{array}{l}\text { Nurse walks into } \\
\text { the room to the } \\
\text { infant crying in the } \\
\text { swing. The nurse } \\
\text { leans down to talk } \\
\text { to the infant and } \\
\text { take the infant out } \\
\text { of the swing. The } \\
\text { nurse says, "Okay } \\
\text { sweet boy, it is not } \\
\text { time for you to eat, } \\
\text { so we need to get } \\
\text { you back to sleep } \\
\text { for awhile." The } \\
\text { nurse looks at the } \\
\text { baby and talks with } \\
\text { the baby as she re- } \\
\text { swaddles the infant } \\
\text { and carries the baby } \\
\text { to a PCA to hold } \\
\text { until it is time for } \\
\text { the infant to feed. }\end{array}$ & $\begin{array}{l}\text { Nurse walks into } \\
\text { the room and states, } \\
\text { "how is my boy } \\
\text { doing?" The nurse } \\
\text { looks at the baby } \\
\text { and says, "Oh, I } \\
\text { know why you are } \\
\text { mad. You need } \\
\text { your diaper } \\
\text { changed." While } \\
\text { the nurse is } \\
\text { changing the diaper, } \\
\text { she is looking at the } \\
\text { baby and telling the } \\
\text { baby "let's get this } \\
\text { stuff off of you, } \\
\text { then you will feel } \\
\text { better. I am } \\
\text { hurrying." The } \\
\text { nurse puts the } \\
\text { pacifier in the } \\
\text { baby's mouth } \\
\text { before the diaper } \\
\text { change. The baby } \\
\text { is looking at the } \\
\text { nurse as the nurse is }\end{array}$ \\
\hline
\end{tabular}




\begin{tabular}{|c|c|c|c|}
\hline $\begin{array}{l}\text { to get the baby to } \\
\text { smile by making } \\
\text { eye contact and } \\
\text { talking quietly to } \\
\text { the baby. The baby } \\
\text { smiles. The nurse } \\
\text { laughs and says, "I } \\
\text { knew that smile was } \\
\text { in there." }\end{array}$ & $\begin{array}{l}\text { bottom is patted } \\
\text { while lying across } \\
\text { the cuddler legs. } \\
\text { The nurse squats } \\
\text { down to look at the } \\
\text { baby and says, "oh, } \\
\text { this feels better." }\end{array}$ & $\begin{array}{l}\text { mouth. After the } \\
\text { infant calms down, } \\
\text { the nurse } \\
\text { reswaddles the } \\
\text { infant in the } \\
\text { blanket, keeping the } \\
\text { pacifier in the } \\
\text { mouth. The nurse is } \\
\text { talking to the baby } \\
\text { and looking at the } \\
\text { baby throughout. }\end{array}$ & $\begin{array}{l}\text { talking and starting } \\
\text { to calm down. } \\
\text { After the diaper } \\
\text { change, the nurse } \\
\text { swaddles the baby, } \\
\text { picks up the baby } \\
\text { and sways and pats } \\
\text { the bottom of the } \\
\text { baby in order to } \\
\text { continue to help the } \\
\text { infant calm down. } \\
\text { The nurse starts } \\
\text { singing a song to } \\
\text { the baby. }\end{array}$ \\
\hline
\end{tabular}


The nurse's role satisfaction involved the subthemes of nurturer or comforter and becoming an expert and were observed during participant observation and described during interviews. The cyclical nature of these two subthemes demonstrate how a nurse who is nurturing and comforting to an infant over time becomes an expert in that infant's care; both of which are extremely meaningful and satisfying to the nurse. This culture of care to infants with NAS requires a strong desire in nurses to provide nurturing and comforting care to infants but who also desire to become experts in an infant's care.

\section{Theme 4: Grief}

Although the infants with NAS in NICU 3 are hospitalized, they are not premature or critically ill. Therefore, the nurse caring for an infant with NAS is usually not concerned with an infant who is on the verge of death. NICU nurses who care for critically ill infants cope with grief for the loss of the infant's life and grieve also with the parents in this loss (Catlin \& Carter, 2002). The grief that is experienced with the nurse who cares for infants with NAS was described as "grief for today" and "grief for the future." Although this is a smaller theme, it was still powerful for these nurses.

Although each nurse expressed grief in some way through participant observation and individual interviews, each nurse expressed grief differently. Most of the nurses expressed grief for when the infant was experiencing multiple withdrawal symptoms with increased Finnegan scores. Also, many nurses verbalized grief for the loss of parental contact for the infant while in the hospital. Participant 02 stated that it was "just hard seeing them here, seeing nobody here to see them, visit them or care for them." It was verbalized by nurses in interviews and during observations when there was a "lack" of parental care for the infant while the infant was 
withdrawing in the hospital and also a grieving for the infant's "loss of bonding with the parents.”

Regarding "grief for the future" the nurses verbalized a "worry" and "concern” for the future home environment for the infant. Many of the nurses expressed concern for the evaluation process of the family situation and the resulting decisions of with whom the infant would go home. The nurses admitted and were aware that there were "many factors about the families' personal lives" that they did not know about and that the "future placement" of the infant depended on these factors. Also, the nurses expressed "powerlessness" to help change the future for the infant going to a possibly unstable home environment. This grief for the future was verbalized by every nurse interviewed in response to the question, "what hardships do you personally experience in caring for infants with NAS?” Participant 04 describes her feelings of "success" with these words, "it warms my heart to know that these babies go home with parents that I know are gonna do well with them, that's a success.”

"Grief for the future" also is based upon the fact that a few of the infants discharged to the home environment have actually died in the home. Many nurses verbalized these facts during participant observations. Participant 08 describes one of these incidents;

When you have to send the baby home with somebody, you know, who is not bonding with the baby, not looking into the child's eyes, they never smile, they are not okay and the baby gets sent home with them and she ends up dead. She was a bitty thing and we loved her and the kid died. We've since had three kids die, and it's awful every single time.

Because the nurses know of these deaths that have happened in the past, there continues to be grief, worry, and fear for the infant who may be going home to a similar home environment. 
One NICU 3 nurse, who previously worked in NICU 1, grieved the "loss of parental contact" while in the NICU and in the future. She stated that previously she had developed strong relationships with parents while they cared for their sick or premature infant in the hospital and desired this "same relationship" with the parents on NICU 3. This nurse stated that because this in-hospital relationship with the nurse and parents "does not occur as often in NICU 3 as in NICU 1," the "future relationship" that nurses may have with parents is diminished as well. Participant 10 stated her grieving in this way:

The part I don’t really like or enjoy is that we don’t really get to see the outcome as opposed to when you work up in the main NICU, a lot of those parents will bring those babies back in. In the NICU 3, the parents aren't bringing the babies back for visits unless they were adopted babies and then occasionally they'll bring them back and that's a very good feeling because then you see something positive. Not to say that if they don't go back with the biological families, it's a not a positive thing, it’s just you don't get to , I wish there was a way that we could see more positives because I think if we could, you would get more nurses wanting to work down here. We want to see a positive outcome. "Grief for today" encompassed the loss of normal newborn life as evidenced by severe withdrawal symptoms and the loss of or "lack of parental contact" that could bring comfort to the infant by the process of "bonding." "Grief for the future” encompassed fear, "concern” and "worry" for the infant's possibly unstable home environment and fear of the possibility of the infant dying at home in an unstable home environment. Overall, the "grief for today" and the perceived loss or "grief for the future" of the infant was verbalized daily during participant observations and in 11 of the 12 interviews. 


\section{Theme 5: Making a Difference}

Many of the previous themes addressed the how in providing a culture of care for infants with NAS, but it is also important to discuss the why in the motivation of these nurses. The most stated phrase by interviewed nurses in answer to the questions "How did you become a part of the core team of nurses who work in NICU 3?" and "What are the successes that you experience in working with these infants?” was making a difference in the lives of the infants. In the theme of making a difference, the subthemes of wonderful insanity, which are the rewards and challenges of care and critical to them emerged to more fully describe this why of care.

Wonderful Insanity (rewards and challenges). All of the NICU 3 nurses stated in interviews and during observations that they have a "passion for babies" and a "love for babies" that motivates them to work with infants. Also, all of the nurses stated that they desired to be a "part of the infant's life” and desired to help them "withdraw” from maternal substances.

The phrase wonderful insanity was a phrased voiced by Participant 08 in relation to the challenge and then reward of caring for an infant with NAS.

You cannot help but love them and I don't know why. I don't know what it is about them that makes them so special to my heart, but it's not just me. The nurse who works down there full time, all say the same things. There's something about them that just captures your heart. I don't know if it's because they try so hard. I don't know if they struggle so much that you root for them like rooting for the underdog. I don't know if it's because their family background is such that you have to cheer for them, hoping against hope that they will be the one that rises above their background. I don't know if it's because when they finally settle, I mean got this kid who just ungulates in your arms. He’s just, they're just in motion, in motion, in motion and it not a happy motion. They’re in motion 
because they're uncomfortable and all of a sudden, you can feel it. They'll go (sigh) and they'll go limp in your arms and you think 'Thank God. Ok I got this one under control’ and it may have been after hours and you may have to carry that kid around most of the shift because they just don't settle, or maybe you had to leave em in the crib and they had to scream most of the shift cause you don't have the hands for it, but when you finally get that peace, that peacefulness and you can see it on their faces, maybe that's what it is that draw you into this type of stuff.

The challenge of the fussing "in motion" infant who cannot be calm and the resulting reward of seeing and experiencing this infant "settle" in the nurse's arms along with the "rooting for the underdog" all exemplified the phrase wonderful insanity to this nurse. This nurse stated in the interview that the complex level of care she provided to these infants was wonderful and insane.

When asked in interviews, "What are the challenges of caring for an infant with NAS?" almost every nurses stated the challenge of "working with parents" in their cycle of addiction. Most of the nurses stated that this was one of the obstacles to caring for the infants. In regards to the parents being a "barrier" the nurses saw the "lack of parental presence" or "not being there" as a barrier to the infant's withdrawal. Another challenge stated by many nurses in observations and during interviews was when a parent arrived on the unit "intoxicated" in some way. The nurses stated that it was difficult to "keep the baby safe" if parents were intoxicated and holding their infants. Nurses stated that these challenges with parents created "stress" and a "lack of knowing what to do" with nurses as they care for the infants. The rewards stated by nurses were when parents made choices to "be a part of their infant's life", "stay in the unit" to care for the infant, and seek rehabilitative services. 
Another challenge or reward that nurses voiced was that of the "newness of the unit." Because NICU 3 is the only unit of its kind, there are no other nursing units to contact to ask how care is given to infants with NAS. Participant 04 expresses the challenge of this new unit in these words.

It's very exciting, to be at the forefront, its' very depressing that we are at the forefront. I can’t imagine not being able to have the knowledge and the technology to work with these babies and with these parents, and you know I really have to give a hands up, a high five to the practitioners that have worked hard in doing so much research on all of this. We have a pharmacist here who has put just countless hours into researching the different narcotics and you know the reactions, its just ongoing. It's never just a lot of research this last year, and this is 'we're gonna stop here and just do this.' It just continues on and we're finding different things everyday that make a difference in these babies' lives. It's just very exciting to be able to be a part of it all.

The nurses are aware of the challenges in caring for these babies and see the "efforts from physicians, nurse practitioners, and the pharmacist” that are all working with nurses to provide the most effective and up-to-date care for these infants. Nurses stated in interviews that "protocols" for care change quickly and this in itself can be a challenge for nurses. However, nurses also stated that when the "new protocol" worked, the challenge was "worth it."

The rewards of caring for infants with NAS are many and were described in these words by nurses, "when the babies get better," "bonding with the infant,” " part of their success,” being a part of "babies overcoming their beginning," "beginning to act normal,” and "well enough to go home.” Participant 01 states about this reward in these words. 
When you see it working that your helping the babies, you get so much back in return, when you work and you have these babies and then, you know, you're doing all this and you see things start to work their scores are doing better, you just don't know what to say, but feelings of relief or just 'oh good it worked,' because these babies are just going through such a torment, you know and you see and find something that works, you're just happier than ever because you know what works with one baby is not gonna work with another baby but you see that that because these babies need whatever relief they can get. Another statement about making a difference by Participant 04 was, "when I see the difference in day and night with these babies when they first come and when they do smile and they do respond like a baby that's appropriate for that age.” Participant 08 described this reward in caring for the infant in these words. "When you can calm a baby, calm that kid down who's having a really bad day and you can get them to clam down, that's nice. When they fall asleep on your chest and you can feel them melt into your body, you can actually feel them melt into you, that's nice."

All of these statements reflect that the care that is provided to these infants, regardless of the challenge, is rewarding to the nurse. The challenges of the infant's withdrawal and their extreme withdrawal symptoms, the challenge of parents, the challenge of being the only unit of its kind with frequently changing protocols and research, bring rewards to the infants, which in turn is rewarding to the nurses. These challenge or reward experiences describe the wonderful insanity of caring for these infants, and the nurses know that these are part of their making a difference in the lives of these infants.

Critical to them. The other part of making a difference that was observed and stated by these nurses was the belief that their "extra care" made a difference with these infants. The 
nurses believe that this kind of "extra care" that is provided while they learn the baby is critical to the success of the infant's withdrawal. Although the nursing care for infants with NAS is not critical care in the same way as in NICU 1, this kind of nursing care is extremely specialized. Participant 011 provided this description of care for infants with NAS.

Maybe it's not critical in the sense of 'my baby's not on a ventilator,' but it's critical to them, you know they hurt, you know they're in pain when they're screaming that something hurts, and they can't do anything about it. The can’t go take medication on their own. They have to depend on us to interpret what's going on with them and decide what they need. Now they're gonna let us know if it works or not, but you know they're just little people and how would you feel if you were gong through what they're going through, and what we do is critical with them, you know, it makes a difference as to what their day is gonna be like.

Many of the nurses expressed that "all of the hard work was worth it" in relation to the challenges of caring for infants with NAS. During observations, many nurses demonstrated the actions of bathing, talking to the infant, singing to the infant, rocking the infant, and expressions of affection. Participant 04 stated, "The care is nurturing care. I just feel like I can probably see these babies respond as much to a warm bath and warm pajamas and a soft bed as they can a higher dose of morphine.” Another nurse stated that "these babies need my patience” which described the "extra care" that is provided. The motivation of these nurses to make a difference by the critical and specific care that is provided during the hospital stay is "Just the care that I give em while they're here.” The nurses know that there are limits to the care that they can give, but while the infant is in the hospital, their motive is to make a difference for the time that they have now. 
Regardless of the kind of care that a nurse provides a patient, it is important for the nurses to know why they care for their patients. During the investigation into the motives of care, beliefs are uncovered that explain the why of care. The belief that these nurses were making a difference was the over-arching theme for the culture of care that they provided to infants with NAS.

\section{Communication}

In this study the communication between nurses was described as "effective" and "supportive." Also, nurses stated in interviews that the care they provided to infants was based on "shared decision-making" and communicating "what worked for the infant." Although this unit provided examples of effective, and at times wonderful communication among nurses, during participant observation and individual interviews, the nurses felt that "if this communication was increased between providers and interdisciplinary staff”, this communication would be even more effective. It was the belief of these nurses that if there was increased communication of new information in the form of "new protocols" and the "decisions for placement of infants after discharge” to the home or foster care setting, that this communication would "benefit the infants," their families, and the nurses who care for them.

\section{Summary}

Results of this ethnographical research revealed five themes that were observed during participant observations and described through individual interviews. These five themes were learn the baby (routine care, comfort care, environment, adequate rest and sleep, feeding), core team relationships (support, interpersonal relationships), role satisfaction (nurturer or comforter, becoming an expert), grief, and making a difference (wonderful insanity, critical to them). These five themes encompassed the codes and categories from participant observation, 
field notes, and individual interviews. Cultural description of care and four of the five nonpharmacological nursing interventions were revealed as subthemes (environment, adequate rest and sleep, feeding, and nurturer or comforter). A discussion of these results follows with general recommendations for the nurse who cares for infants with NAS. 


\section{CHAPTER 5}

\section{DISCUSSION}

\section{Introduction}

The purpose of this research was to identify and describe the culture of care and nonpharmacologic nursing interventions performed by NICU nurses while providing care to infants with NAS. Although every infant is different, my goal was to provide direction to the nurse caring for infants with NAS; therefore, there are general recommendations for environment, adequate rest and sleep, feeding, and the role of the nurse nurturer or comforter. This focused ethnography consisted of participant observation and individual interviews with 12 NICU 3 nurses that offered rich descriptions of the culture of care provided to infants. This focused ethnography used the five steps of data analysis as outlined by Roper and Shapira (2000) and revealed five themes. The themes describe the how and why of culture in the form of nursing interventions with the potential for practical application to nursing practice. Five major themes emerged from analyzing field notes and interview data. Four of these major themes had subthemes. These themes and subthemes were,

1) learn the baby (routine care, comfort care, environment, adequate rest and sleep, feeding),

2) core team relationships (support, interpersonal relationships),

3) role satisfaction (nurturer or comforter, becoming an expert),

4) grief, and

5) making a difference (wonderful insanity, critical to them).

Each theme is presented below in relation to culture of care.

In this study, culture was earlier defined as behavioral (patterns of behavior) and 
cognitive (what they know and believe) (Roper \& Shapira, 2000). By applying these cultural concepts of how and why values to the five themes, the culture of NICU 3 was described. Earlier, care or caring was defined as intentional and holistic care to infants. Each theme is discussed in relation to the culture (how and why) and care or caring. Although the two attributes of culture and the one attribute of care or caring are defined separately, these attributes are intimately connected and portrayed in the five themes. Lastly, four themes is discussed in relation to the nonpharmacological nursing interventions.

\section{Discussion of Themes}

Theme 1: learn the baby (subthemes: routine care, comfort care, environment, adequate rest and sleep, feeding)

The how. The first theme that emerged from the analyzed data was learn the baby. This major theme included the five subthemes of routine care, comfort care, environment, adequate rest and sleep, feeding. This theme reflected the nurses' patterns of behavior in relation to the daily care provided to the infants. Routine care is the care that all nurses provide to infants with NAS every 3 hours. The impact of the routine care structure provides extended observational time with focused observations on a limited set of important physical changes in the infant. Also, because routine care is every 3 hours, it allows the nurse to observe increases and decreases in withdrawal symptoms.

These nursing interventions are learned when more experienced nurses teach the newer nurses how to perform them. Great value is placed on nurses performing this structured set of interventions that are the basis of care for an infant with NAS. For example, every nurse in individual interviews mentioned how vital routine care was to the care of the infant. Although the interventions used during routine care are formulaic and structured, how the nurse performs these interventions is individualized to the infant. Usually, before an infant is captured, the 
interventions are performed very gently and carefully to not over-stimulate the infant. After the infant is captured, routine care becomes a structure for continued assessment and a way to look for improvements. The importance of routine care for infants with NAS provides a foundational approach to the assessment of the infant with NAS.

Comfort care is the care that can be provided to infants with NAS through individual nursing interventions or in combinations of nursing interventions to help relieve withdrawal symptoms. These comfort care nursing interventions can be provided during routine care and between routine care. Once learned, the nurses shared knowledge with not only less experienced nurses but also among caregivers that know how to care for these infants. An example of this was with the nurse 05 who frequently used the Halo swaddle to swaddle her infants; she was frequently found helping other nurses secure their infant in the Halo swaddle as well. However because there were no evidence-based protocols or clear rules to guide the application of the nursing interventions, nurses used a trial-and-error approach to comfort care for infants experiencing extreme fussiness. The familiar phrase on the unit, learn the baby, was the way the nurses expressed the intentional learning of an infant's behavior and "what worked" to calm the infant.

The subthemes of environment, adequate rest and sleep, and feeding are also part of the way a nurse learns the baby. Each of these nursing interventions involved previous knowledge that a NICU nurse would have developed from caring for premature or sick infants. However, the process of learning the individual needs of infants with NAS (learn the baby) required the NICU nurse to make adaptations to NICU care to meet the individual needs of the infant. Each of these subthemes was depicted in observational charts of 12 nurses. For providing an “environment" of comfort, a subtheme, interventions included decreasing the light and noise in 
the infant's immediate surroundings and also decreased the amount of touch the infant experienced. These interventions were most common before the infant was captured. All of the nurses stated that the process of learning the baby allowed the nurse to understand what kinds of environmental stimuli an infant could handle to remain or become calm. The infant's tolerance levels to environmental stimuli were described by Participant 09, "they have to learn how to deal with the stimuli but it stresses ‘em them til they're captured.” For the NICU in general, a separate area for infants with NAS, private rooms, or rooming in with the mother and infant could provide even more detailed results on the external environment of light, noise, and touch and its effect on infants (Abrahams, 2007; Murphy-Oikonen et al., 2012).

For adequate rest and sleep, all of the nurses performed swaddling in some way. Nurses routinely swaddle infants to mimic the comfort of the womb and prolong sleep. This intervention also decreases the Moro reflex, which can wake or aggravate an infant (Franco et al., 2004; Gerard, Harris, \& Thach, 2002). Most nurses were observed swaddling infants with a blanket, while two of the nurses used a Halo swaddle. Even within the process of using the Halo swaddle, each of the nurses performed this intervention differently; one with the infant's arms and hands midline before the swaddle was secured while the other with the infant's arms placed in an "arms flexed, fist up" position. The nurse with the specialized technique with the Halo swaddle shared her expertise across the entire unit. To promote sleep, most of the fussy infants were held and over half of the infants were rocked and a pacifier was used. Also, most infants were placed first in the crib, but if the infant could not remain asleep, the infant was placed in the swing. Many nurses held a rationale for making crib sleep a preference because the nurse was thinking about the infant's future and the kind of equipment likely available in the home. Although some infants could not tolerate the crib and needed to be rocked by the nurse or placed 
in the swing, nurses, knowing what the infant needed, would first try the crib. All of the nurses stated that the process of learning the baby allowed the nurse to understand what the infant needed to sleep or rest in between feedings.

For feeding, most of the nurses swaddled the infant during the feeding in some way. In order to prevent vomiting, many of the nurses burped the infants frequently instead of half way through to prevent vomiting. Because of the infant's uncoordinated suck, NICU 3 nurses all used a strategy of providing chin and cheek support to help the infant coordinate suck and swallow, which was verified during interviews. Chin and cheek support would have been a nursing strategy used with premature and sick infants in the NICU because these infants require chin and cheek support because they are immature and weaker than a newborn. However, infants with NAS have an uncoordinated suck due to their withdrawal from maternal substances. The infant's poor feeding or uncoordinated suck is one of the scored components of the Finnegan scoring tool. All of the nurses stated that the process of learning the baby allowed the nurse to understand the specific knowledge about chin and cheek support and frequent vomiting in regards to feeding most effectively. A list of general recommendations for environment, adequate rest and sleep, and feeding is provided at the end of this section and is based on the data analysis. These recommendations are a starting point for the behaviors and patterns of care that are required for these infants. The articulation of the how in clinical nursing practice is crucial to the provision of expert nursing care (Benner, 2004).

The why. Much of the theme learn the baby was about the how (patterns of behaviors) of nursing care for infants with NAS. However, in the midst of routine care, comfort care, environment, adequate rest and sleep, and feeding, nurses believe that this care made a difference in the infant's life. Nurse 10 stated this in regards to the success she experienced in 
caring for infants with NAS, "when an infant overcomes their beginning and becomes more normal.” Participant 04 stated this answer to why she works with these infants, "It just continues on and we're finding different things everyday that make a difference in these babies' lives. It's just very exciting to be able to be a part of it all.” Also, there is a belief that when the nurse learns the baby the nursing interventions that are performed to fit the baby will help to decrease the withdrawal symptoms these infants experience. More of the why of nursing interventions are discussed in the theme making a difference.

Care or caring component. It was generally acknowledged, observed, and stated that the nurses from NICU 3 were all passionate about the infants they cared for. When nurses are passionate about a specific population of patients, they will demonstrate more caring feelings and behaviors toward these patients. Intentional caring affects the nurse's response to the patient and transforms the knowing and actions from general patterns of care to specific and individual patterns of care. This nurse response of caring develops and evolves over time as caring acts are practiced and understood through multiple care experiences (Boykin \& Schoenhofer, 2001a). Therefore, as a nurse learns the baby over time, the nurse continues to respond to the infant with what previously worked to decrease withdrawal symptoms. Learning the baby with its individualized patterns of care illustrates these points in the literature.

\section{Recommendations for the environment in providing a culture of care for infants with}

NAS. All of the nurses were observed decreasing the amount of light and noise and limiting the amount of touch used with the infant with NAS. This set of interventions was used extensively during the period before the infant was captured when the withdrawal symptoms were at their worst. Participant 01 stated, “you need to treat the babies like they have a 300\% migraine.” Nurses also stated in interviews that the individual rooms for each infant helped to decrease light, 
noise, and touch. This description of the infant with a "migraine" helped nurses realize the extreme sensitivity that these infants have to light, noise, and touch. This nurse also stated that the private room for each infant helped with this to provide decreases light, noise, and touch. As with any nursing intervention involving infants with NAS, every issue of discomfort and how the infant is comforted depends on the needs of the infant. All of the nurses stated that the process of learning the baby allowed the nurse to understand what kinds of environmental stimuli an infant could handle to remain or become calm. If one intervention, such as turning on the music from the aquarium, did not work, they tried another until the infant became calm. Also, even though the nurses decreased environmental stimuli for the infants, after the infant was captured, they would try to "open the blinds during the day and the keep room dark at night" in an effort to help the infant with future adaptations. Listed in Table 9 are recommendations for the nonpharmacological nursing intervention of environment before the infant is captured.

Table 9

Recommendations for the Environment of the Infant Before Captured

\section{If an infant is fussy}

Light: Before captured-keep room dark

Noise: Before captured-quiet room

White noise from the music mobiles or noise from the swing

Touch: Before captured—little touch or touching only when needed Firm touch or firm holding Rhythmic patting

\section{Recommendations for adequate rest and sleep in providing a culture of care for infants}

with NAS. General recommendations for adequate rest and sleep are listed in Table 10. All of the nurses were observed swaddling the infant in some way. Most of the fussy infants were being held and over half of the infants were rocked and a pacifier was used. Also, most infants were 
placed first in the crib, but if the infant could not remain asleep, the infant would be placed in the swing. In order for newborn infants to gain weight, it is important for them to sleep between their feedings (Tikotzky et al., 2009). Nurses on NICU 3 would know this from their previous experiences of caring for premature and sick infants in the NICU. However, adequate rest and sleep for infants with NAS was difficult because of the discomfort of their withdrawal symptoms. All of the nurses interviewed stated that the process of learning the baby allowed the nurse to understand what the infant needed to sleep or rest in between feedings. If one intervention, such as the use of a pacifier did not work, they tried another until the infant was calm enough to rest or sleep. Knowing that infants needed to sleep in order to gain weight, nurses would try to swaddle the infant, hold and rock the infant, or use the swing if the infant would not stay asleep in the crib. Nurse 7 stated this desire with adequate rest and sleep, "promote a home setting for rest." Nurses placed great value on this nursing intervention and would try multiple comfort measures to help the infant sleep.

Table 10

Recommendations for the Nurse to Help Provide Adequate Rest and Sleep

To promote adequate rest and sleep

Swaddling of some kind
Holding the infant
Rocking the infant
Use of a pacifier if infant has an organized suck desires
Placed in the crib first, then the swing

Recommendations for feeding in providing a culture of care for infants with NAS. All of the nurses were observed bottle-feeding the infant with NAS. An interesting finding during these observations of feedings was that 8 of the 12 infants were calm or sleepy at the beginning of the feeding. It was unknown at this time if the infants were captured or uncaptured. Most of 
the nurses swaddled the infant during the feeding in some way. In order to prevent vomiting, many of the nurses burped the infants frequently instead of half way through the feeding and at the end of the feeding. Because food is not always a comfort measure it is wondered if the frequent burping is one measure that the nurse did to expel the extra air that is taken in with bottle-feeding because the infant is already experiencing cramping and diarrhea.

Many of the infants required chin and cheek support; this was also verified during many of the interviews. This action on the part of nurses to provide chin and cheek support while feeding was a NICU skill that was transferred to the infant with NAS although the need for the interventions was different; NICU infants have a weak or immature suck and infants with infants with NAS have an unorganized suck due to withdrawal symptoms. As the infant progressed chin and cheek support was less needed as the infant's suck became more organized. With this kind of individualized care, if one intervention, such as holding the infant close did not work, the nurse would try to hold the infant away from her body. General recommendations for feeding. are listed in Table 11.

Table 11

Recommendations for Feeding Feeding

Swaddle the infant Chin and cheek support if needed Frequent burping

With the nonpharmacological nursing interventions of environment, adequate rest and sleep, and feeding, the nurses' goals were to try to minimize withdrawal symptoms before the infant was captured (when withdrawal symptoms are most severe). Although pharmacotherapy is part of the infant's treatment for withdrawal symptoms, the nurse was always trying to keep environmental stimuli decreased and provide adequate rest and sleep to promote weight gain and 
feed the infant in a manner that caused the least amount of gastrointestinal upset. The nurse accomplished this by learning the baby.

\section{Theme 2: core team relationships (subthemes: support and interpersonal relationships)}

The how. The theme of core team relationships with the subthemes of support and interpersonal relationships provided a rich intertwining of the assistance needed among nurses in caring for a fragile population of infants while at the same time providing close personal relationships between the core team nurses. Overall, the observed and verbalized group patterns of behavior reflected a mutual sustaining or support in caring for the infants. Nurses offered to help each other, solve problems together, listened to each other as they discussed an infant, and applauded and grieved the progress of the infants together. An example of this was when Nurse 10 said to Nurse 9, "let me know what you find out from social work about the baby.” Nurses asked questions and kept each other "up to date" on the progress of the infants in a genuine atmosphere of caring. As these behaviors were demonstrated on the unit, they became the norm of behavior; a culture of care consisted of caring for infants and caring for each other. These relationships among the core team of nurses not only helped and supported the nurses while they cared for the infants with NAS, but also, became personal and close in their interpersonal interactions with each other. In the process of caring for infants, they cared also for each other. Table 12 describes just some of these specific supportive behaviors among NICU 3 nurses. 
Table 12

Supportive Behaviors of NICU 3 Nurses

\section{Supportive Behaviors}

Touch on greeting each other

Throughout the day, updating each other about the self

Questions asked about personal lives

Laughing, joking, teasing

Offering to help each other care for their infants

Helping each other with medication discrepancies

Throughout the day, updating each other about the infants

The why. Throughout observations and individual interviews, the core team nurses made reference to themselves as a "group.” The characteristics of the core team of nurses were that they previously cared for premature and sick infants, have nurturing personalities in regards to infants and have a tendency toward expressing relationship freely. They expressed that they needed each other to not only physically care for the infants but to understand each other's emotions as they cared for the infants. When there was an infant demonstrating increased withdrawal symptoms and the nurses spoke to each other about their concerns for the infant, they used words and expressions to describe their concern that were understood in relation to the care of the infant. An example of this from observations was during change-of-shift report, Nurse 11 sitting next to Nurse 8 and reporting details of her three infants with statements such as, "You know how he cries," "his excoriation on his face is getting better," and "the heal warmer to his belly helped him to sleep.” A phrase that was part of the shared vocabulary of NICU 3 nurses was the phrase stated in all interviews, "learn the baby.” Nurses learned and believed that they could express their concerns in a mutually understood and accepted atmosphere. Nurses frequently were observed giving each other pats on the back and hugs during the workday. This physical and emotional support during nursing care led to the development of close 
interpersonal relationships among them. During interviews three of the nurses referred to themselves "like a family" and one nurses stated they were a "close knit group of girls." Support and interpersonal relationships were not mutually exclusive, but were meshed in nurses' conversations and expressions throughout observations and interviews by conversations about families, vacations, life activities outside of work, and personal issues as well as the infants they cared for. Therefore, these actions and beliefs demonstrated some characteristics of a family because of the mutual goal of making the "family" unit work by the support of their interpersonal relationships. However, these actions and beliefs are also characteristics of close friendships where mutual talking, listening, and helping each other continue to strengthen the bonds of relationships.

Care or caring component. Although job satisfaction varies in different areas of nursing, it has long been supported by research that job satisfaction among nurses is related to interpersonal relationships between nurses and patient care (Jackson, 2005; Utriainen \& Kyngäs, 2009). Caring is central to nursing and is demonstrated by individual and holistic care to patients (Watson, 1988). Some of this same holistic care was observed in how the nurses cared for each other by support (in caring for infants) and by interpersonal relationships (in caring for each other). When asked the question "what makes work easier for you?” the response most stated was "working with the core team." These nurses have a built-in support system in their work environment for the intense work they are performing. This "working toward the same goal" in regards to the comfort of the infants gave these nurses a shared support in reaching these goals. Also in regards to coping and how nurses support each other, Participant 09 stated, "you just get the babies through as well as you can and you just focus on what you can do.” This phrase, "focusing on what you can do," was stated by nurses often during participant observation and in 
interviews but also was a statement that the nurses used to support each other during a difficult day.

This stable core team of nurses provided support and interpersonal relationships that proved satisfying and increased job satisfaction in a stressful work environment. Schmalenber and Kramer (2007) report that intensive care nurses and especially NICU nurses experience more healthy and productive work environments. For high stress areas of nursing care, it is recommended that hospital systems seek nurses who volunteer for placement in specific areas. Also, it is recommended that NICUs providing care for infants with NAS, pursue nurses who desire constant contact with infants with NAS. These nurses develop expertise in their care and are then the ones to pass this nursing knowledge along to other nurses. This provision of a core team of nurses, consistency of care to the infants and a culture of support and interpersonal relationships can lead to units where nursing job satisfaction is high

\section{Theme 3: role satisfaction (subthemes: nurturer or comforter, becoming an expert)}

The how. Role satisfaction was an important theme to the nurses on NICU 3. The subthemes of nurturer or comforter and becoming an expert both described the aspects of their role that they found most important. The nurses on NICU 3 used the terms nurturer and comforter interchangeably, which is why each term was used for this subtheme. Both terms represent a kind of special care that is given to infants with NAS. Infants with NAS require nurturing because they are all newborns and also comforting because they exhibit withdrawal symptoms that can cause irritability and discomfort. Nurses who possess nurturing and comforting qualities find satisfaction in providing nurturing or comforting care to these infants. This "giving away" of nurture and comfort to infants with NAS was meaningful to these nurses and part of the why for this culture of care. Recommendations for the nurturer or comforter role 
in providing a culture of care for infants with NAS are provided at the end of this section.

The how of nurturing and comforting an infant with NAS was described by the core team nurses as enjoyable care, hands on care, physical care, emotional care, intense care, and developmental care. During individual interviews the nurses described their role with the infant as one who genuinely loves the family like a long lost family member, protector, advocate, their support system, caretaker, nurse, medical support, the one who gives them a good start, educator, in the middle, fire fighter, social worker, police, director of a day care, and surrogate mommy (but not take the place of their mommy). Although the descriptions of their role varied, each nurse understood this role in the context of providing emotional and physical nurturing and comforting. As the nurse performed routine care and comfort care with nurturing and comforting behaviors, the nurse became an expert in the care of that infant. Becoming an expert by learning the baby was important to the nurse in how they viewed themselves in their role and their satisfaction with their role. Nurses demonstrated these behaviors seamlessly in the context of care. These patterns of care were taught to other nurses and exemplified.

The why. The cyclical nature of the two subthemes nurturer or comforter and becoming an expert demonstrated how a nurse who is nurturing and comforting to an infant over time becomes an expert in that infant's care; both of which were extremely meaningful and satisfying to the nurse. This relates to the recommendation that was made earlier regarding staffing, because several of these emerged themes are factors that would not have been developed without the "core team" type of assignment. Over time, the nurse's consistency of care with the same infant provided the opportunity to develop expertise in the care of the infant. The core team was crucial to the care of the infants with NAS.

This belief that nurturing or comforting behaviors was needed towards infants with NAS 
while they withdraw from maternal substances was apparent in these nurses. All of the nurses believed that this kind of care, which can lead to becoming an expert, was crucial for the infant's withdrawal. Without nurturing or comforting, the nurses believed that the infants would have higher Finnegan scores and require more pharmacotherapy to help with withdrawal. Therefore, this culture of care to infants with NAS required a strong desire in nurses to provide nurturing or comforting care to infants, but who also desire to become experts in an infant's care.

Care or caring component. In order to provide nurturin or comforting care, the nurse has to have a strong personal desire to care holistically for the infant. The intentional care provided by the nurse to observe and understand what the infant needs to be comforted leads to becoming an expert. Nurses, as caring individuals, were satisfied with their role as nurses when they saw that they had become experts in the infant's care with the result of the infant's withdrawal.

Recommendations for the nurturer or comforter role in providing a culture of care for infants with NAS. Of course there is an overlap of the nurturer or comforter role in providing comfort to a fussy infant with the comfort care nursing interventions. The nurses did not rely on a list of nursing interventions to try with an infant but intuitively used many of them simultaneously; if one intervention did not work, they tried another until the infant became calm. General recommendations for nurturing or comforting behaviors from the nurse to the infant with NAS are listed in Table 13. 
Table 13

Recommendations for Nurturing or Comforting Behaviors from the Nurse to the Infant

\section{If an infant is fussy}

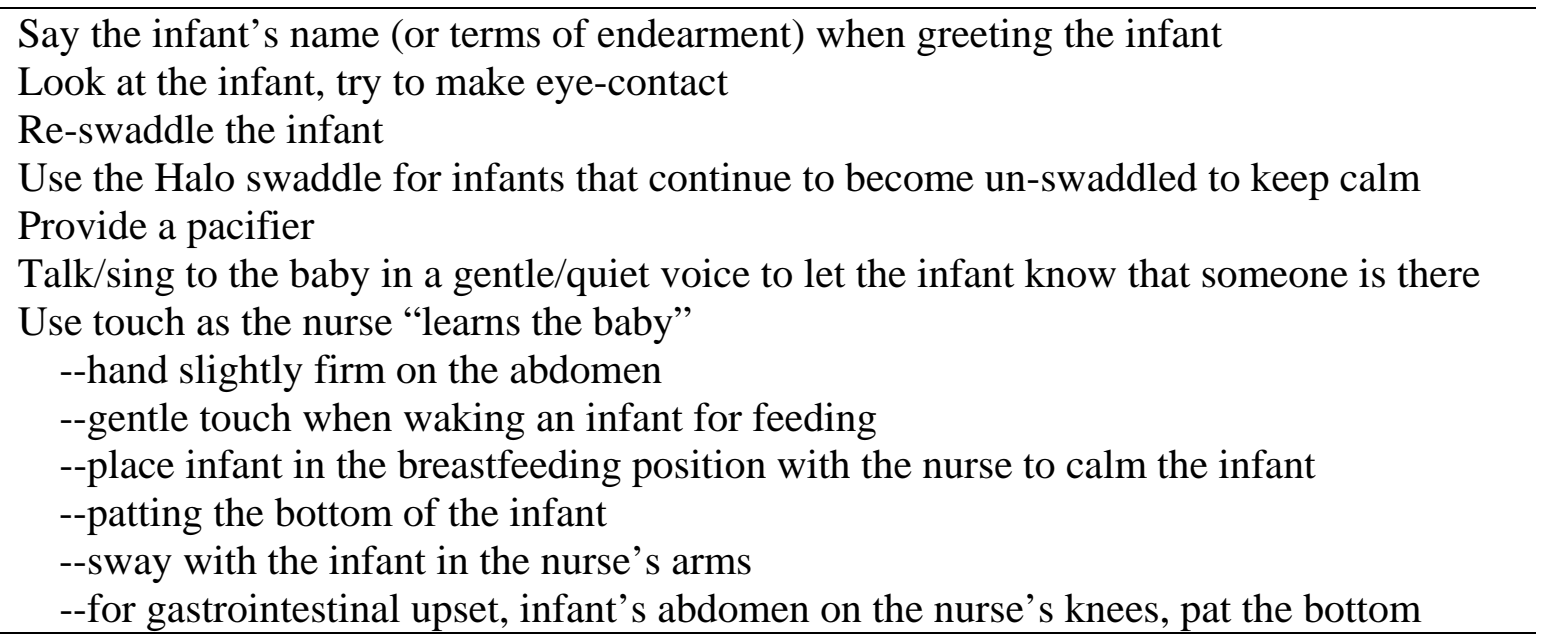

\section{Theme 4: grief}

The how. The theme labeled as grief was another important element of the culture of care in this unit. The "grief for today" and "grief for the future” were expressions and feelings of real and perceived loss that was experienced daily in NICU 3. Nurses learned that expressing their concerns about the infant's current withdrawal symptoms, concerns for the infant's future health outcomes, and the home environment was allowed and encouraged as a means of support. When the nurses verbalized their concerns, and these concerns were validated by other nurses, there was shared and substantiated grief.

The why. The NICU 3 nurses expressed their grief for the infants openly, being mindful of parents and confidentiality. The belief that this grief could be expressed in the form of words, feelings and even tears was "freeing” to the nurses. The "grief for today” was discussed whenever an infant was extremely fussy and having difficulty remaining calm and the "grief for 
the future" was discussed often amongst the nurses. This future grief was also communicated to physicians, nurse practitioners, and social workers.

Cultural care component. Grief would not occur if there was no caring on the part of the nurses. Nurses experienced sadness and loss in regards to the severity of withdrawal symptoms infants were experiencing, the lack of a normal newborn life and potential bonding with parents. Also, nurses were sad about the possible future home environment based on the previous experiences of past infants. Nurses in the caring role, try to prevent loss by providing a culture of care for these infants. Grief is experienced when a nurse cannot prevent this loss. Research with neonatal grief in the literature encompasses the grief that is experienced when NICU nurses experience the loss of life of a premature or sick infant (Kati $\tilde{A}^{\circ}$ scia Vergutz Diel, Calcagno Gomes, Modernel Xavier, dos Santos Salvador, \& Minasi de Oliveira, 2013).

However, infants with NAS do not usually die. The grief that these nurses experience pertains to loss of a "normal" newborn life as perceived by the nurse. The concept of grief is highly individualized (Cowles \& Rodgers, 1991), however, there is a question as to the expressions of real grief or perceived grief and if they are determined by the socialization of a group? So the question must be asked, were the verbalizations of "grief for today" and "grief for the future" somehow socially constructed? On NICU 3, these nurses inherently acknowledged that grief was expected and a part of this culture. Nurses expressed grief openly and knew that grief was part of what they do, even though these infants do not normally die on the unit. As a culture these nurses accept this. To date there is no research on the effects of grief on NICU nurses who care for infants with NAS.

It is common knowledge that most hospital centers provide counseling services for healthcare personnel who have experienced the loss of a patient. I did not observe or hear in 
interviews that any of these nurses pursued counseling to deal with their grief. A question arises to the thought that maybe these NICU 3 nurses offer support to each other in such a way that no outside support or counseling is needed.

Many of the nurses expressed concern for the evaluation process of the family situation and the resulting decisions of whom the infant would go home with. The evaluation process included many individuals from social work , the Department of Child Services (DCS), physicians, and nurse practitioners. The nurses admit and were aware that there were many factors about the families' personal lives that they did not know about and that the future placement of the infant depended on these factors. Regardless of the presence or absence of the parents and their personal stories, there was great concern from the nurses for the present and future lives of the infants based on previous experiences.

\section{Theme 5: Making a Difference (subthemes: wonderful insanity, critical to them)}

The how. A powerful theme expressed by NICU 3 nurses, making a difference, encompassed the two subthemes of wonderful insanity and critical to them. The phrase and subtheme wonderful insanity exemplified the rewards and challenges of caring for infants with NAS. The phrase and subtheme critical to them characterized the type of critical care that was and was not needed for these infants. Any nurse who would consider working on a unit with infants with NAS would immediately know that this kind of work would be challenging. So, why do nurses work with these infants? Why also, do NICU trained nurses with highly technical skills decide to work in a less technical area with extremely fussy and irritable infants with complex social situations? The answer to these two question was that these nurses knew that they could make a difference in the lives of these infants. The reward is that the infant does withdraw and the nurses know that they are part of this success in helping the infant. This wonderful insanity 
(tension between rewards and challenges) was part of the reason that these nurses knew that despite the challenges, rewards would occur for these infants. Critical to them was a description of the kind of care that these infants need. Infants with NAS do not need ventilators, umbilical lines, and specialized surgeries; these infants need frequent holding, being talked to, swaddling and reswaddling, rocking, singing, the pacifier provided, bouncing, a swing, special kinds of touch, chin and cheek support with feeding, frequent burping, dark and quiet rooms, and most of all patience. These patterns of care are the type of "critical" care that these infants need. The nurses learn these patterns from each other and, in turn, provide a culture of care for these infants that will help the infants to withdraw from maternal substances. The nurses learn from each other and support each other in this intentional and holistic care. Together, as a team, they make $a$ difference by providing a culture of care and nurturing/caring environment.

The why. Besides knowing how to provide a culture of care for infants with NAS, the NICU 3 nurses also believe that they can personally make a difference in the lives of the infants. The nurses know and believe that the rewards will follow the challenges of caring for these infants. The nurses learn how to perform routine care, comfort care, decrease environmental stimuli, how to provide adequate rest and sleep, and how to feed these infants. The nurses were willing to experience the wonderful insanity of care in order to make a difference. Many of the NICU 3 nurses were trained as NICU nurses with highly technical skills. Although these skills are important for premature and sick infants, the NICU 3 nurses know and believe that the other skills that are critical to the babies are critical to them. When this nurturing or comforting culture of care is provided to infants with NAS, the nurses believed they were making a difference. Although the nonpharmacological nursing interventions provided by NICU nurses to infants with NAS are crucial to their care and withdrawal, the literature only briefly mentions 
them and few are empirically tested (D’Apolito, 1999; Velez \& Jansson, 2008). The potential contribution of nonpharmacological nursing interventions in managing withdrawal should not be underestimated. The articulation of clinical nursing practice in the form of protocols, procedures, and clinical practice guidelines lead nurses in the provision of expert nursing care (Benner, 2004). Placing value on nonpharmacological nursing interventions allow nurses to make a difference.

Care or caring component. Watson's (1985a) definition of caring depicts the nurse providing individualized care to patients. When a nurse who is caring for an infant with NAS provides individualized and holistic care, the nurse will make a difference in the infant's life. Although there will be rewards and challenges (wonderful insanity), this individualized care will help decrease withdrawal symptoms in these infants. Also, the individual care that is critical to them, in the form of routine care and comfort care, will make a difference as well. Regardless of the level or intensity of care for these infants by nurses, effective caring promotes health and healing (Watson, 1979).

Discussion of Themes in Relation to the Research Question: What is the culture of care and nonpharmacologic nursing interventions performed by NICU nurses who provide care to infants with NAS?

The emerged themes explained the knowledge and beliefs that were observed on NICU 3 and were related to the research question: What is the culture of care and nonpharmacologic nursing interventions performed by NICU nurses who provide care to infants with NAS? These connections between themes and the research question help to make sense of the rich and complex data. Theme one, learn the baby, was described as the process of learning the individual comfort measures needed to provide care and comfort for the infant. Another way that the theme, learn the baby, was explained was the intentional learning of the baby's personality. The process of learning the baby occurs through routine care, comfort care, and the nonpharmacological 
nursing interventions of environment, adequate rest and sleep, and feeding. In order for the nurse to learn the baby, the nurse learned many patterns of how (behavioral concept of culture) to care for the infant with NAS from the other nurses on NICU 3. The why (cognitive concept of culture) of care for the infants relates to theme five making a difference. The challenges of the infant's withdrawal and their extreme withdrawal symptoms, the challenge of parents, the challenge of being the only unit of its kind with frequently changing protocols and research, bring rewards to the infants, which in turn is rewarding to the nurses. These challenging or rewarding experiences described the wonderful insanity of caring for these infants, and the nurses knew that these were part of making a difference in the lives of these infants.

The role of the nurse with the subthemes of nurturer or comforter and becoming an expert speak to the caring component that is central to nursing (Watson, 1979). Before a nurse can learn the baby and in turn make a difference, the nurse must first exhibit humanness in the receiving and giving of care and love. This care is demonstrated and practiced by responding to others as unique individuals (Watson, 1985a). The nurturer or comforter role was needed to respond to individual infants with NAS. As the nurse learned how to nurture and comfort the infant, the nurse becomes an expert in the infant's care.

One of the greatest challenges of caring for infants with NAS is the nurse's grief for today and grief for the future. This grief was part of the cycle of rewards and challenges presented earlier. Also, in order to process this grief the core team relationships were needed. The core team provided support for the rewards and challenges of caring for these infants, but at the same time provided interpersonal relationships for the nurses, which were deeply satisfying. These five identified themes describe the how and why of the culture of care provided by NICU nurses to infants with NAS. 


\section{The Meaning of the Culture of Care in NICU 3}

The descriptions of the culture of care and the interpretation of the data are entrenched in the researcher's knowledge and understanding of the participants in NICU 3. According to Roper and Shapira (2000) nurses are "naturals" at ethnography. Although I am a novice researcher, I brought a specific familiarity of the research population (cuddler and previous NICU nurse) and was able to make inferences. Intense engagement in the field through participant observation, individual interviews and the examination of existing documents were all part of the data collection and analysis, which resulted in triangulation. All of these methods gave the researcher a better knowledge of the population enhancing her ability to make meaning from the data.

The larger body of literature is scarce in relation to culture of care within the context of caring for infants with NAS. However, Wilson, McCormack, and Ives (2005) in a study of culture in a special care nursery (NICU) found that nurses experienced teamwork in that "good democratic leadership made this a positive place to work" (p. 30-31). They also found that "staff are there to help you" and would "listen to what you have to say" (p. 31). However, in this study, there was a contrast between those who had a positive experience in the unit with those that felt tensions amongst the staff. This sample included all nursing staff $(\mathrm{N}=27)$ (Wilson et al., 2005). My findings did not coincide with the positive or negative experiences as the Wilson et al. (2005) study. This could be due to the fact that I was observing and interviewing core team nurses who chose to leave the technologically advanced area of the NICU to work in a critical to them (no ventilators and no umbilical lines) area of the NICU. Also, my study was with core team nurses only which is a specific population of nurses and is supported by my method (Roper \& Shapira, 2000).

One of the questions that I asked in relation to the limited literature on culture of care and 
NICU nurses who care for infants with NAS is this, is the culture influenced more positively with a voluntary population of nurses? The core team of nurses in my study chose to work on NICU 3. Many of these nurses felt like they had a "specific love for these babies" and a “calling” to work with these babies; therefore, do these nurses experience increased role satisfaction over other nurses in a NICU who care for premature, sick, and infants with NAS all in the same physical space? This role satisfaction of nurses who care exclusively for infants with NAS needs further study. However, my research indicated that there was great satisfaction in their role as nurses in this context.

Mefford and Alligood's (2011) study evaluating staffing patterns in the NICU asked this question, does consistency of nursing caregivers make a difference in health and economic outcomes? The results of this study indicated greater levels of consistency of care in the NICU and influenced decreased length of stay and shorter durations of mechanical ventilation. Although this study was conducted in a NICU with premature and sick infants, there may be some correlation with the consistency of care in NICU 3 with decreased length of stay and decreased withdrawal symptoms with infants with NAS.

The Brown et al. (2003) research among four NICU units and the factors that increase the culture of collaboration found that NICU environments "cannot exit without trust and respect among and between all team members” (p. e482). Also in this study the researcher found that “disagreements and differences” of opinion could provide a stimulus for change. With so many changes occurring quickly on NICU 3 due to changing weaning protocols and advances in the care for infants with NAS, these “differences” or in the words of the NICU 3 nurses from interviews, “communication barrier of rationales for care decisions” could provide useful for changes in care. Changes in care and collaboration influence culture. This study adds to the 
current body of literature surrounding the meaning and descriptions of culture provided by NICU nurses to infants with NAS because this specific research is not discussed in the literature.

Figure 1 visually depicts the researcher's explanations and interpretations of the concepts explored in this research. The conceptual model provides a description of the themes of this research and how they relate to the infant with NAS. 


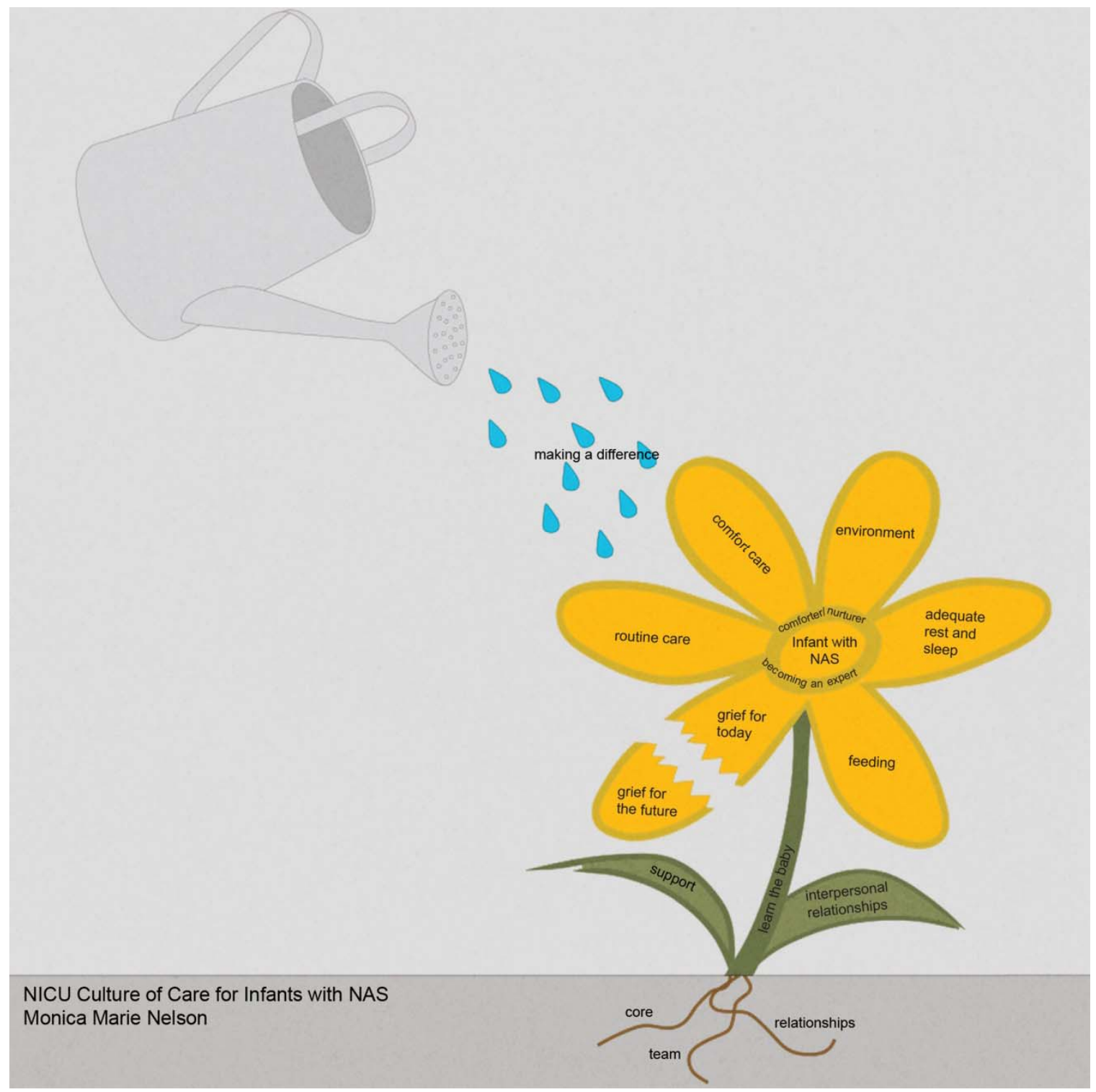

Figure 1.Conceptual model of the NICU culture of care for infants with NAS. The infant with NAS is the center of the flower with all culture and interventions focused on the infant. Five of the petals (routine care, comfort care, environment, adequate rest and sleep and feeding) are subthemes of the theme learn the baby. The theme of core team relationships is represented by the roots of the flower and each of the leaves, which are the subthemes of support and interpersonal relationships. The theme of role satisfaction is represented by the ring around the infant, which include the subthemes of comforter or nurturer and becoming an expert. The theme of grief is represented by the petal that is still attached to the flower (grief for today) and the petal that is separated from the flower (grief for the future). The theme of making a difference is represented by the water from the watering can. 


\section{Implications for Nursing}

In an effort to describe the implications for nursing for the culture of care provided by NICU nurses for infants with NAS, the following areas are discussed: implications for nursing theory, implications for nursing research, and implications for nursing education. Each section is by no means exhaustive but rather an identification of major current themes.

\section{Implications for Nursing Theory}

Implications of findings supported by Watson's theory of caring. Caring is central to nursing (Watson, 1979, p. 8-9). Watson's theory of human caring defines caring as the way that nurses provide care to their patients. Responding to each person as a unique individual, the caring nurse perceives and responds to the specific needs of patients (Watson, 1985a). For the purposes of this paper, care or caring was defined as intentional and holistic care to infants to infants. The researcher believes that Watson's theory of caring provides understanding of the individual care provided to infants with NAS through the themes of learn the baby (routine care, comfort care, environment, adequate rest and sleep, feeding), core team relationships (support, interpersonal relationships), role satisfaction (nurturer or comforter, becoming an expert), grief, and making a difference (wonderful insanity, critical to them).

Although this hospital setting does not use Watson's caring theory as a framework for nursing practice, the concepts of care or caring were observed clinically. Nurses did benefit from responding to patients through caring behaviors by recognizing and responding to others as unique individuals, which promoted a process of caring for infants with NAS (Watson, 1985a). This process of caring for infants with NAS was represented in the theme learning the baby and was typical care in this in-patient setting. Because this hospital setting promotes family-centered care, this institution and others seeking a holistic approach to nursing care could easily 
implement Watson's theory of caring. For example, as NICU nurses are trained and oriented to work in the NICU, educational programs can teach and practice Watson's framework of caring from preceptor to learner. Although the NICU can be an intense clinical setting with increasing complex technology, the implementation of a caring framework can keep nurses grounded in the reasons that they care for infants.

Implications of findings supported by Kolcaba's theory of comfort. Kolcaba's theory of comfort is a middle range theory that is useful for clinical nursing practice in a variety of specific populations. Kolcaba (2001) states, "Comfort is defined as the state that is experienced by recipients of comfort measures. It is the immediate and holistic experience of being strengthened through having the needs met for the 3 types of comfort (relief, ease, and transcendence) in four contexts of experience (physical, psychospiritual, social, and environmental)" (p. 90). This theory uses a taxonomic structure and a comfort questionnaire to design interventions and measure them for the outcome of comfort.

Kolcaba's first published article was An Analysis of the Concept of Comfort using the Walker and Avant method (1991). Four meanings of the concept of comfort were discovered during this analysis. The first meaning of comfort is the actual "cause of relief from discomfort and/or being in a state of comfort” (Kolcaba \& Kolcaba, 1991, p. 1302). The second meaning is a peaceful or contented state. These first two meanings represent a cause and effect relationship. Causes of comfort produce relief from discomfort. The third meaning of comfort is the relief from discomfort. This relief may be complete, incomplete, or deliver only partial comfort. Relief may not be the complete absence of discomfort. The fourth meaning focuses on "whatever makes life easy or pleasurable” (Kolcaba \& Kolcaba, 1991, p. 1302). The fifth and sixth meanings are derived from Latin etymology and are obsolete in ordinary language and mean to 
strengthen greatly or give encouragement. These last meanings indicate causes of renewal, positive mindsets, and physical refreshment. All of the meanings of comfort discovered by Kolcaba reflect an improvement from a previous state or condition. Through the process of concept analysis, Kolcaba found that the concept of comfort could be a verb, noun, adjective, or process (outcome).

Although this hospital setting does not use Kolcaba's theory of comfort as a framework for nursing practice, the concepts of comfort by the subthemes of comfort care and nurturer or comforter was observed clinically. Infants with NAS benefited from the comfort provided by nurses by their nonpharmacological and pharmacological (not stated in this study) nursing interventions of comfort. The use of Kolcaba's theory of comfort with the care of infants with NAS could prove useful for measuring "relief from discomfort" and the phases of comfort being “complete, incomplete or partial.” Kolcaba has developed a General Comfort Questionnaire can be adapted for use with a specific population. The empirical precision of the theory of comfort has been researched and proven effective with women with breast cancer, patients with urinary incontinence, pediatrics, perianesthesia patients, and advanced directives (Kolcaba, 2003). At this time there is not a comfort questionnaire for infants with NAS, but van Dijk, Peters, van Deventer and Tibboel (2005) have developed a tool for neonates, infants, and critically ill children. The use of this tool in collaboration with the Finnegan scoring tool could prove useful in measuring comfort from withdrawal symptoms with these infants.

\section{Implications for Nursing Research}

This study provided general guidelines and recommendations for four of the five nonpharmacological nursing interventions (environment, adequate rest and sleep, feeding, Finnegan scoring, and nurses as caregivers). Environment, adequate rest and sleep, feeding, and 
the role of the nurse (nurturer or comforter) were all observable nursing interventions that were discussed in the themes with general recommendations provided for the nurse.

Recommendations for environment, adequate rest and sleep, feeding, and role of the nurse (nurturer or comforter) were provided in Tables 9, 10, 11, and 13. Although the four nonpharmacological nursing interventions of environment, adequate rest and sleep, feeding, and the role of the nurse (nurturer or comforter) yielded general recommendations to the nurse, these nursing interventions need further study with different hospital settings to further describe the meanings of these interventions.

In regards to the culture of care that nurses provide to infants with NAS, this study provides implications in the form of themes that describe the nurses' experiences in caring for infants with NAS. Nurses described the how of this culture of care by the theme of learn the baby. The subthemes of learn the baby (routine care, comfort care environment, adequate rest and sleep, and feeding) provide descriptions of real care to infants with NAS. The why of this culture of care emerged in the theme of making a difference. The subthemes of making a difference (wonderful insanity and critical to them) provide descriptions about the beliefs of nurses and why they desire to care for these infants. The other themes of core team relationships and grief bring added meanings to this study. The core team relationships on the unit offered support to the nurses as they care for infants with NAS as well as interpersonal relationships, which seemed to strengthen the support that the nurses provide to each other. As nurses express grief in the form of "grief for today" and "grief for the future" the nurses support each other and provide the strength of interpersonal relationships that are needed to sustain this type of nursing care. Understanding the how and why of the culture of care provided by nurses brought a depth and richness to the interpretation of the results. 
Research involving infants with NAS, pharmacotherapy for these infants, the effects of individual rooms versus the care of infants with NAS alongside premature and sick infants in the NICU, breast feeding infants with NAS, types of formula for bottle feeding to decrease gastrointestinal disturbances, the use of swaddling, length of stay, occupational therapy, and physical therapy are just a few of the areas that are needed for research with these infants. Because the incidence of NAS has increased, so must the research involving these infants and their care.

Other areas of research that are needed include the long-term effects of maternal substances on the neurological and behavioral development of these infants as they grow and mature. Research is also needed on the appropriate therapies to minimize developmental delays. As these infants approach school age, researching possible testing strategies for the undiagnosed child will be needed. Teachers will need to have evidenced-based practices for teaching and learning with these children in public and private schools.

Continued research with the nurses and institutional healthcare personnel who care for these infants is needed to ensure that caregivers are provided with best practices for these infants. Clinical practice guidelines need to be researched and updated frequently. A few other important and needed areas of research are infants with NAS and bonding with their mothers, the mother's addiction, and how best to facilitate the mother's recovery. Decreasing the gap between clinical practice and evidence-based practice by the use of researched protocols is needed. Further research is also needed for the nonpharmacological nursing interventions for environment (light, noise, and touch), adequate rest and sleep, feeding (formula and breastfeeding), assessment and evaluation using Finnegan scoring by nurses, and the role of the nurse. 
It is much easier for the recommendations for nursing to take precedence over the implications for nursing. Recommendations are much neater, tidier, and give the nurse a "to do" list of nursing interventions. Implications may provide answers, but they also lead researchers to new questions. Implication in qualitative research can provide insight, direction, and awareness to a specific research site at a specific time. Implications also encourage more research to develop richer and more complex data (Quirk Enterprises, 2014). This study provided both recommendations (Tables 10, 11, 12 and 13) and implications for this healthcare facility and the nurses who care for infants with NAS.

\section{Implications for Nursing Education}

With the increased numbers of infants with NAS, more education in Pediatrics and in Obstetrics is needed in the class and clinical setting. Community and Psychiatric nursing will need to address the issues of addiction and the community agencies that will become healthcare providers for these infants and their mothers. Nursing education will also need to address the growth and development needs, assessment, and therapies of these infants as they grow and mature. These newborns will become toddlers, preschoolers and school-age children and will require educated and passionate healthcare personnel to care for them and their developmental needs. Licensure exams for nursing and the advance practice nurse will need to incorporate testing for this added educational content into schools of nursing curriculum. Forbes and Hickey (2009) speak to the "lag" between current practice and revised NCLEX content that contributes to the increasing gap between education and practice.(p. 8). In a study by Morgan and Knox (1987) and later replicated by Nehring (1990), both found that the best clinical nursing teachers were those who exhibited expert clinical skill and judgment. In a time where there is a shortage of nursing faculty in nursing schools and also an increasing average age of nursing faculty, 
clinical educators can be lacking in expertise and clinical judgment. This poses a problem to the graduate nurse entering the profession of nursing. This lag can leave nursing students with a need for increased critical thinking skills in the clinical setting (Penz \& Bassendowski, 2006). Nursing education needs to address how to keep clinical nursing instructors current with clinical practice and how to test current evidence-based practices concerning infants with NAS.

\section{Limitations}

This was a qualitative study. Qualitative studies are contextual in nature. Therefore, the findings are not generalizable. However, rich data and descriptions allow readers to consider transferability of these to a similar context. This focused ethnography was conducted at a hospital in the southeast United States. The study took place in a NICU setting that was exclusively for the care of infants with NAS; therefore, the results may or may not be transferable to a general NICU environment. Also, the drugs taken by mothers could be a regional concern; therefore, in another setting, the withdrawal symptoms could be different in infants with NAS. How a nurse cares for an infant with different withdrawal symptoms could lead to varying results.

\section{Personal Reflections on the Research Process and the Results}

As a novice researcher, I have reflected on the current method for my future research endeavors. These "I wish that I knew then what I know now" items were in the areas of participant observation and interviewing. After compiling the observation charts for environment, adequate rest and sleep, and feeding, I wish that I had devised the charts before observation instead of compiling them after the data were collected. Also, in the area of interviewing, I saw that I improved my interviewing skills with each interview. During my first interviews, I was so concerned with getting all of the questions asked that I may have missed 
those deeper questions that can be asked from the initial question; those questions that expand on a brief statement spoken by the participant help to provide even deeper meanings

In order for the quality of data analysis and writing to become the "measure of usable observational data” (Denzin \& Lincoln, 2000, p. 676) I had to be continually mindful of my researcher bias. I am not sure that any qualitative research can be conducted without some subjectivity. My past experiences and immersion as a NICU nurse sometimes clouded the richness of data collected because these interventions and environment were common to me. I felt like my role as a "cuddler” previous to this research helped me to gain rapport and entrance to NICU 3. The nurses already knew that I enjoyed holding the infants and being on the unit. Also, the nurses know that I will be returning to my "cuddler” role and know that I did not become a cuddler to gain entrance but to truly be a part of helping these infants. Because I have been a NICU nurse in the past, these nurses knew that they could discuss technical and medical issues with the shared language and experiences that comes from being a NICU nurse. My reflective journal was crucial to this research in that is allowed me to make my experiences, opinions, thoughts, and feelings visible and an acknowledged part of the research design, analysis, and interpretation process. However, the effect of keeping and using a reflective journal went beyond achieving methodological rigor and paradigmatic consistency. Critical selfreflection had an effect on the research process; changes were made to the research design, methods used, and approaches taken. An example of this was during one of the early interviews, which a nurse shared her feelings of grief for the future home environment of an infant she was caring for. I had begun to experience feelings of attachment to this infant a few weeks before, so when the nurse began to share these same feelings, it helped me to understand even more the depth of feeling and commitment that these nurses have for these infants. 


\section{Conclusion}

This focused ethnography of the NICU culture of care provided to infants with NAS examined nurses' knowledge and beliefs about the care they provide to these infants. Data analysis of the field notes from participant observation and 12 transcribed interviews with NICU 3 nurses revealed five themes. The themes were learn the baby (routine care, comfort care, environment, adequate rest and sleep, feeding), core team relationships (support, interpersonal relationships), role satisfaction (nurturer or comforter, becoming an expert), grief, and making a difference (wonderful insanity, critical to them).

Participants presented the emic view of the culture of care. In order for the nurse to learn the baby, the nurse learned many patterns of how (behavioral concept of culture) to care for the infant with NAS from the other nurses on NICU 3. The why (cognitive concept of culture) of care for the infants relates to theme five making a difference. The challenges of the infant's withdrawal and their extreme withdrawal symptoms, the challenge of parents, the challenge of being the only unit of its kind with frequently changing protocols and research, brought rewards to the infants, which in turn was rewarding to the nurses. These challenge or reward experiences described the wonderful insanity of caring for these infants, and the nurses know that these are part of making a difference in the lives of these infants.

The role of the nurse with the subthemes of nurturer or comforter and becoming an expert speak to the caring component that is central to nursing (Watson, 1979). Before a nurse can learn the baby and then in turn make a difference, the nurse must first exhibit humanness in the receiving and giving of care and love. This care is demonstrated and practiced by responding to others as unique individuals (Watson, 1985a). The nurturer or comforter role is what is needed to respond to individual infants with NAS. As the nurse learns how to nurture and 
comfort the infant, the nurse becomes an expert in the infant's care.

One of the greatest challenges of caring for infants with NAS is the nurse's grief for today and grief for the future. This grief is part of the cycle of rewards and challenges presented earlier. Also, in order to process this grief, the core team relationships were needed. The core team provided support for the rewards and challenges of caring for these infants, but at the same time provided interpersonal relationships for the nurses, which were deeply satisfying.

The etic view of the researcher was to link what I observed with what I was told and to make interpretations based on these findings. My goal was to identify the nurses’ meanings reflexively by critically reflecting on my own biases, preferences, and preconceived ideas about the care of these infants. Although I was influenced by the experiences, I tried to separate myself from the data as an outsider (etic view). This reflexivity, through self-awareness and introspection, enriched the quality of the study. My goal was to bring the insider and outsider view to this ethnographic report and provide deeper insights than the nurses alone or the researcher alone (Roper \& Shapira, 2000).

The results of this ethnographic research provided descriptions of the culture of care from NICU nurses to infants with NAS. Also, the results of this study helped provide general recommendations to the nurse caring for an infant with NAS in the areas of environment, adequate sleep and rest, feeding, and the role of the nurse (nurturer or comforter). This study has implications for future study of evidence-based research strategies to decrease withdrawal symptoms in infants with NAS. Research is also needed in the areas of clinical practice guidelines to help the bedside nurse care for these infants with current research and evidence for practice. Nursing theory and nursing education both contribute to nursing research in how to better understand the culture of care provided by nurses and to reduce the gap in nursing 
knowledge. Nurses who care for infants with NAS need to be encouraged and applauded for their passion and commitment to the excellent culture of care they provide. Marcellus (2007) states, "The level of knowledge, skill, patience, and commitment required to provide excellent nursing care to this group of infants should not be underestimated” (p. 38). 


\section{REFERENCES}

Aamodt, A. M. (1991). Ethnography and epistemology: Generating nursing knowledge. In J. M. Morse (Ed.), Qualitative nursing research: A contemporary dialogue (pp. 40-54).

Thousand Oaks, CA: Sage.

Abdel-Latif, M. E., Pinner, J., Clew, S., Cooke, F., Lui, K., \& Oei, J. (2006). Effects of breast milk on the severity and outcome of neonatal abstinence syndrome among infants of drug-dependent mothers. Pediatrics 117(6), e1163 - e1169.

Abrahams, R. R., Kelly, A., Payne, S., Thiessen, P. N., Mackintosh, J., \& Janssen, P. A. (2007). Rooming-in compared with standard care for newborns of mothers using methadone or heroin. Canadian Family Physician, 53, 1722-1730.

AbuAlRub, R. F. (2004). Job stress, job performance, and social support among hospital nurses. Journal of Nursing Scholarship, 36(1), 73-78.

Aita, M., \& Snider, L. (2002). The art of developmental care in the NICU: A concept analysis. Journal of Advanced Nursing, 41(3), 223-232.

Alligood, M. R., \& Marriner-Tomey, A. (2010). Nursing theory: Utilization \& application (4 ${ }^{\text {th }}$ ed.). Maryland Heights, MO: Mosby Elsevier.

Als, H. \& Lester, B., Tronick, E. Z., \& Brazelton, T. B. (1982). Manual for the assessment of preterm infants' behavior (APIB). In H. E. Fitzgerald, B. M. Lester \& M. W. Yogman (Eds.), Theory and research in behavioral pediatrics, 1, 35-63. New York, NY: Plenum Press.

Als, H., Lawhorn, G. Brown, E., Gibes, R., Duffy, F. H., McAnulty, G. \& Blickman, J. G. (1986). Individualized behavioral and environmental care for the very low birth weight preterm infant at high risk for bronchopulmonary dysplasia: Neonatal Intensive Care Unit 
and developmental outcome. Pediatrics, 78(6), 1123-1132.

Als, H., Duffy, F. H., McAnulty, G. B., Rivkin, M. J., Vajapeyam, S., Mulkern, R. V., Warfield, S. K., Huppi, P. S., Butler, S. C., Conneman, N., Fischer, C., \& Eichenwald, E. C. (2004). Early experience alters brain function and structure. Pediatrics, 11(4), 846-857.

American Academy of Pediatrics. (1998). Neonatal drug withdrawal. Pediatrics, 101(6), 1079 1088.

American Academy of Pediatrics. (2001). The transfer of drugs and other chemical into human milk. Pediatrics, 108(3), 776-789.

American Academy of Pediatrics. (2005). Breastfeeding and the use of human milk. Pediatrics, 129, e827.

American Academy of Pediatrics. (2011). Policy statement: SIDS and other sleep-related infant deaths: Expansion of recommendations for a safe infant sleeping environment. Pediatrics, 128(5), 1029-1039.

American Academy of Pediatrics. (2012a). Policy statement: Patient- and family-centered care and the pediatrician’s role. Pediatrics, 129(2). Retrieved January, 2014 from: doi:10.1542/peds.2011-3084

American Academy of Pediatrics. (2012b). Breastfeeding and the use of human milk: Section on breastfeeding. Pediatrics 129, e827.

American College of Obstetricians and Gynecologists and the American Society of Addiction Medicine. (2012). Opioid abuse, dependency and addiction during pregnancy. Committee opinion No. 524. Obstetrics and Gynecology, 119(5), 1070-1076. 
Andrasik, M. P.,Chandler, C., Powell, B., Humes, D., Wakefield, S., Kripke, K., \& Eckstein, D. (2014). Bridging the divide: HIV prevention research and black men who have sex with men. American Journal of Public Health, 104(4), 708-714.

Backes, C. H., Backes, C. R., Gardner, D., Nankervis, C.A., Giannone, P.J., \& Cordero, L. (2012). Neonatal abstinence syndrome: Transitioning methadone-treated infants from an inpatient to an outpatient setting. Journal of Perinatology, 32, 425-430.

Bakstad, B., Sarfi, M., Welle-Strand, G. K, \& Ravndal, E. (2009). Opioid maintenance treatment during pregnancy: Occurrence and severity of Neonatal Abstinence Syndrome. European Addiction Research, 15,128-134.

Ballard, J. L. (2001). Treatment of Neonatal Abstinence Syndrome with breast milk containing methadone. Journal of Perinatal and Neonatal Nursing, 15(4), 76-85.

Becker’s Healthcare. (2012). 10 guiding principles for patient-centered care. Retrieved from http://www.beckershospitalreview.com/quality/10-guiding-principles-for-patientcentered-care.html

Benner, P. (2004).Designing formal classification systems to better articulate knowledge, skills and meaning in nursing practice. American Journal of Critical Care, 13(5), 426-429.

Bialoskurski, M., Cox, C. L., \& Hayes, J. A. (1999). The nature of attachment in a neonatal intensive care unit. Journal of Perinatology and Neonatal Nursing, 13(1), 66-77.

Boykin, A., \& Schoenhofer, S. O. (2001a). Nursing as caring: A model for transforming practice. Sudbury, MA: Jones \& Bartlett.

Brinkman, M. A. (2013). A focused ethnography: Experiences of registered nurses transitioning to the operating room. (Order No. 3570586, Widener University School of Nursing). ProQuest Dissertations and Theses, 231. Retrieved from 
https://login.ezproxy.etsu.edu:3443/login?url=http://search.proquest.com/docview/14203 57372?accountid=10771

Brown, M. S., Ohlinger, J.,Rusk, C., Delmore, P., \& Ittmann, P. on Behalf of the CARE Group (2003). Implementing potentially better practices for multidisciplinary team building: Creating a neonatal intensive care unit culture of collaboration. Retrieved from http://www.pediatrics.org/cgi/content/full/111/4/e482

Carlson, J. A. (2010). Avoiding traps in member checking. The Qualitative Report, 15(5), 11021113. Retrieved from http://www.nova.edu/ssss/QR/QR15-5/carlson.pdf

Carper, B. A. (1978). Fundamental patterns of knowing. Advances in Nursing Science, 1(1), 1324.

Catlin, A., \& Carter, B. (2002). Creation of a neonatal end-of-life palliative care protocol. Neonatal Network: The Journal of Neonatal Nursing, 21(4), 37-49.

Centers for Disease Control and Prevention. (2013). Guidelines for preventing transmission of Human Immunodeficiency Virus through transplantation of human tissue and organs. Retrieved October, 2013 from http://www.cdc.gov/mmwr/preview/mmwrhtml/00031670.htm

Chinn, P. L., \& Kramer, M. K. (2008). Integrated theory and knowledge development in nursing. St. Louis, MO: Mosby Elsevier.

Clarke, L. (1996). Covert participant observation in a secure forensic unit. Nursing Times, 92(48), 37-40.

Cowles, K. V. (1996). Cultural perspectives of grief: An expanded concept analysis. Journal of Advanced Nursing, 23, 287-294.

Cowles, K. V., \& Rodgers, B. L. (1991). The concept of grief: A foundation for nursing research 
and practice. Research in Nursing \& Health, 14(2), 119-127.

Cruz, E. V., \& Higginbottom, G. (2013) The use of focused ethnography in nursing research. Nurse Researcher, 20(4), 36-43.

D’Apolito, K. (1999). Comparison of a rocking bed and standard bed for decreasing withdrawal symptoms in drug-exposed infants. Maternal Child Nursing, 24(3), 138-144.

D’Apolito, K., \& Hepworth, J. T. (2001). Prominence of withdrawal symptoms in polydrugexposed infants. Journal of Perinatology and Neonatal Nursing, 14(4), 46-60.

D'Apolito K., \& Finnegan, L. (2010). Assessing signs and symptoms of neonatal abstinence using the Finnegan Scoring Tool, an inter-observer reliability program. Neo Advances [online]. Retrieved from http://www.neoadvances.com/program.html

Denzin, N. (1978). The research act: A theoretical introduction to sociological methods. New York, NY: McGraw-Hill.

Denzin, N. K., \& Lincoln, Y. S. (2000). Handbook of qualitative research (2nd ed.). Thousand Oaks, CA: Sage.

Dinges, D., Davis, M., \& Glass, P. (1980). Fetal exposure to narcotics: Neonatal sleep patterns as a measure of nervous system disturbance. Science, 209, 619-621.

Dryden, C. Young, D., Hepburn, M., \& Mactier, H. (2009). Maternal methadone use in pregnancy: Factors associated with the development of neonatal abstinence syndrome and implications for healthcare resources. British Journal of Gynecology (BJOG), 116, 665671.

Duke University Medical Center. (2005). Patient safety-quality improvement: Culture of safety. Retrieved March, 2014 from http://patientsafetyed.duhs.duke.edu/module_c/module_overview.html 
Fawcett, J. (2005). Criteria for evaluation of theory. Nursing Science Quarterly, 18, 131-135.

Federal Guidelines for Opioid Treatment. (2013). Retrieved from

http://www.dpt.samhsa.gov/pdf/FederalGuidelinesforOpioidTreatment5-62013revisiondraft_508.pdf

Finnegan, L. P., \& MacNew, B. A. (1974). Care of the addicted infant. American Journal of Nursing, 74(4), 685-693.

Finnegan, L.P., Connaughton, J., Kron, R., \& Emich, J. P. (1975). Neonatal abstinence syndrome: Assessment and management. Addictive Diseases: International Journal, 2(1), 141.

Finnegan, L.P., \& Ehrlich, S.M. (1990). Maternal drug abuse during pregnancy: Evaluation and pharmacotherapy for neonatal abstinence. In Modern methods in pharmacology (pp. 25566). New York, NY: Wiley-Liss.

Franco, P. Seret, N. Van Hees, J, Scaillet, S. Groswasser, J., \& Kahn, A. (2004) Influence of swaddling on sleep and arousal characteristics of healthy infants. Pediatrics, 115, 13071312.

Fraser, J. A., Barnes, M., Biggs, H. C., \& Kain, V. J. ((2007). Caring, chaos and the vulnerable family: Experiences in caring for newborns of drug-dependent parents. International Journal of Nursing Studies, 44, 1363-1370.

Fricker, H. S., \& Segal, S. (1978). Narcotic addiction, pregnancy, and the newborn. American Journal of Diseases in Childhood, 132(4), 360-366.

Gerard, C.M., Harris, K.A., \& Thach, B.T. (2002). Physiologic studies on swaddling: An ancient child care practice, which may promote the supine position for infant sleep. The Journal of Pediatrics, 141(3), 398-404. 
Gerlach, K. (2010). The effect of breastfeeding on the neonatal abstinence scores of infants born to mothers on a methadone maintenance program: An epidemiological study. (Order No. MR64052). Available from ProQuest Dissertations \& Theses Full Text. (734023587). Retrieved October 2013 from https://login.ezproxy.etsu.edu:3443/login?url=http://search.proquest.com/docview/73402 3587?accountid $=10771$

Germain, C. P. (1979). The cancer unit: An ethnography. Wakefield, MA: Nursing Resources.

Gill, N. E., Benke, M., Conlon, M., McNeely, J. B., \& Anderson, G. C. (1988). Effect of nonnutritive sucking on behavioral state in preterm infants before feeding. Nursing Research, 37,347-350.

Gray, K., Dostal, S., Ternullo-Retta, C., \& Armstrong, M. (1998). Developmentally supportive care in a neonatal intensive care unit: A research utilization project. Neonatal Network, 17, 33-38.

Gray, T. R., Choo, R. E., Concheiro, M., Williams, E., Elko, A., Jansson, L., Jones, H., \& Huestis, M. A. (2010). Prenatal methadone exposure, meconium biomarker concentrations and neonatal abstinence syndrome. Addiction, 105, 2151-2159.

Green, A., McSweeney, J., Ainley, K., \& Bryant, J. (2007). In my shoes: Children’s quality of life after heart transplant. Progress in Transplantation 17(3), 199-207.

Guba, E. G., \& Lincoln, Y. S. (1994). Competing paradigms in qualitative research. In N. K. Denzin \& Y. S. Lincoln (Eds.). Handbook of qualitative research (pp. 105-117). Thousand Oaks, CA: Sage.

Hack, M. (2009). Care of preterm infants in the neonatal intensive care unit. Pediatrics, 123, 1246. 
Harper, R. G., Solish, G. I., Purow, H. M., Sang, E., \& Panepinto, W. C. (1974). The effect of a methadone treatment program upon pregnant heroin addicts and their newborn infants. Pediatrics, 54(3), 300-305.

Higginbottom, G. M. A., Pillay, J., \& Boadu, N. Y. (2013). Guidance on performing focused ethnographies with an emphasis on healthcare research. The Qualitative Report, 18(17), 1-16. Retrieved February 2014 from http://www.nova.edu/ssss/QR/QR18/higginbottom17.pdf

Hill, R. M., \& Desmond, M. M. (1963). Management of the narcotic withdrawal syndrome in the neonate. The Pediatric Clinics of North America, February, 67-85.

Hudak, M. L., \& Tan, R. C. (2012). Neonatal drug withdrawal. Journal of the American Academy of Pediatrics, 12, e540-e560.

Hulatt, J., (2000). Neonatal abstinence syndrome: How and where should babies with this condition be cared for? Journal of Neonatal Nursing, 6(5), 159-164.

Hymes, D. (1972). Models of the interaction of language and social life. In J. J. Gumperez \& D. Hymes (Eds.), Directions in sociolinguistics. New York, NY: Holt, Rinehart \& Winston.

International Lactation Consultant Association (2004). Summary of the hazards of infant formula. Retrieved October, 2013 from http://www.ilca.org/i4a/pages/index.cfm?pageid=3933

Institute of Medicine. (2010). The future of nursing: Leading change, advancing health. Washington, DC: National Academy Press.

Isemann, B. Meinzen-Derr, J., \& Akinbi, H. (2011) Maternal and neonatal factors impacting response to methadone therapy in infants treated for neonatal abstinence syndrome. Journal of Perinatology, 31, 25-29. 
Jackson, C. (2005). The experience of a good day: A phenomenological study to explain a good day as experienced by a newly qualified RN. International Journal of Nursing Studies, 42, 85- 95.

Jacobs, S. E., Sokol, J., \& Ohlsson, A. (2002). The newborn individualized developmental care and assessment program is not supported by meta-analyses of the data. Pediatrics, 141(3), 451-452.

Janesick, V. J. (2000). The choreography of qualitative research design. In N. K. Denzin \& Y. S. Lincoln (Ed.). Handbook of qualitative research (2nd ed.). Thousand Oaks, CA: Sage.

Jansson, L. M., \& Velez, M. (1999). Understanding and treating substance abusers and their infants. Infants and Young Children, 11(4), 79-89.

Jansson, L. M., Velez, M. \& Harrow, C. (2004). Methadone maintenance and lactation: A review of the literature and current management guidelines. Journal of Human Lactation, 20, 62-70.

Jansson, L. M., Choo, R., Velez, M. L., Harrow, C., Schroeder, C. R., Shakleya, D. M., \& Huestis, M. A. (2008). Methadone maintenance and breastfeeding in the neonatal period. Pediatrics, 121, 106-114.

Johnson, K., Greenough, A., \& Gerada, C. (2003). Maternal drug use and length of neonatal unit stay. Addiction, 98, 785-789.

Jones, H. E., Finnegan, L. P., \& Kaltenbach, K. (2012). Methadone and buprenorphine for the management of opioid dependence in pregnancy. Drugs: Current Opinion, 72(6), 647657.

Kaltenbach, K., \& Finnegan, L. (1986). Neonatal abstinence syndrome, pharmacotherapy and developmental outcome. Neurobehavioral Toxicology and Teratology, 8, 353-355. 
Kandall, S.R., \& Gartner, L. M. (1974). Late presentation of drug withdrawal symptoms in newborns. American Journal of Diseases in Children, 127, 58-61.

Karp, H. (2002). The happiest baby on the block. New York, NY: Bantam Dell.

Knoblauch, H. (2005). Focused ethnography. Forum Qualitative Sozialforschung/Forum Qualitative Research, 6(3). Retrieved February, 2014 from http://www.qualitativeresearch.net/fqs-texte/3-05/05-3-44-e.htm

Kolcaba, K. Y. (2003). Comfort theory and practice. New York, NY: Springer.

Korner, A. F., Kraemer, H. C., \& Haffner, M. E. (1975). Effects of waterbed flotation on premature infants: A pilot study. Pediatrics, 56, 361-167.

Korner, A. F., Schneider, P., \& Forrest, T. (1983). Effects of vestibular-proprioceptive stimulation on the neurobehavioral development of preterm infants: A pilot study. Neuropediatrics, 14, 297-299.

Kutlu, A., Memik, R., Mutlu, M, Kutlu, R., \& Arslan, A. (1992). Congenital dislocation of the hip and its relation to swaddling used in Turkey. Journal of Pediatric Orthopaedics, 12,598-602.

Lall, A. (2008). Neonatal Abstinence Syndrome. British Journal of Midwifery, 16(4), 220-224. Lambert, V. Glacken, M., \& McCarron, M. (2011). Employing an ethnographic approach: Key characteristics. Nurse Researcher, 19(1), 17-23.

Landreville, A. (2008). Critical care nurse perceptions and experiences regarding their role in the decision making process to withdraw life-sustaining treatment: A focused ethnography (Order No. MR47170). Available from ProQuest Dissertations \& Theses Full Text. (304409183). Retrieved January 2014 from https://login.ezproxy.etsu.edu:3443/login?url=http://search.proquest.com/docview/30440 


\section{3?accountid=10771}

Lawrence, R. A. (2010). Breastfeeding: A guide for the medical profession (7th ed.). Philadelphia, PA: Saunders.

Leininger, M. (1995a). Culture care theory, research and practice. Nursing Science Quarterly, 9(2), 71-78.

Levy, G. D., Woolston, D. J., \& Browne, J. V. (2003). Mean noise amounts in Level II vs. Level III neonatal intensive care units. Neonatal Network, 22(2), 33-38.

Liaw, J. J., Yang, L., Lee, C. M., Fan, H.C., Chang, Y. C., \& Cheng, L. P.(2013). Effects of combined use of non-nutritive sucking, oral sucrose, and facilitated sucking on infant behavioral states across heel-stick procedures: A prospective, randomized controlled trial. International Journal of Nursing Studies, 50(7), 883-94.

Lim, S., Prasad, M. R., Samuels, P., Gardner, D.K., \& Cordero, L. (2009). High-dose methadone in pregnant women and its effect on duration of neonatal abstinence syndrome. American Journal of Obstetrics \& Gynecology, 200, 70.e1-70.e5.

Lincoln, Y. S. \& Guba, E. G. (1985). Naturalistic inquiry. Beverly Hills, CA: Sage.

Liu, M., Lin, K., Chou, Y. \& Lee T. (2010). Using non-nutritive sucking and oral glucose solution with neonates to relieve pain: A randomized controlled trial. Journal of Clinical Nursing, 11-12, 1604-11.

Long, J. G., Philip, A. G., \& Lucey, J. F. (1980). Noise and hypoxemia in the intensive care nursery. Pediatrics, 65,143-145.

Lucas, K., \& Knobel, R. B. (2012). Implementing practice guidelines and education to improve care of infants with neonatal abstinence syndrome. Advances in Neonatal Care, 12(1), 40-45. 
Ludington-Hoe, S., Hadeed, A., \& Anderson, G.C. (1991). Physiologic responses to skin-to-skin contact in hospitalized premature infants. Journal of Perinatology, 11, 19-24.

Ludington-Hoe, S. M. (2011). Thirty years of kangaroo care and science. Neonatal Network, 30(5), 357-62.

Ludington-Hoe, S. M. (2013). Kangaroo care for neonatal abstinence syndrome: Case studies. Neonatal Network, 32(2), 140-1.

Lyons, S. A. S. (2007). Cultural contexts of continence care: A focused ethnography of two nursing homes. (Order No. 3301726, The University of Iowa). ProQuest Dissertations and Theses, 381-n/a. Retrieved January, 2014 from https://login.ezproxy.etsu.edu:3443/login?url=http://search.proquest.com/docview/30486 0650?accountid=10771

Madden, J. D., Chappel, J. N., Zuspan, F., Gumpel, J., Mejia, A., \& Davis, R. (1977). Observation and treatment of neonatal narcotic withdrawal. American Journal of Obstetrics and Gynecology, 127(2), 199-201.

Maguire, C. M., Walther, F. J., van Zwieten, P. H., Cessie, S., Wit, J. M., \& Veen, S. (2009). Follow-up outcomes at 1 and 2 years of infants born less than 32 weeks after newborn individualized developmental care and assessment program. Pediatrics, 123(4), 1081-7.

Maizes, V., Rakel, D., \& Niemiec, C. (2009). Integrative medicine and patient-centered care. Commissioned for the IOM Summit on Integrative Medicine and the Health of the Public. Retrieved January, 2014 from: http://www.iom.edu/ /media/Files/Activity\%20Files/Quality/IntegrativeMed/Integrative \%20Medicine\%20and\%20Patient\%20Centered\%20Care.pdf

Malpas, T. J., Darlow, B. A., \& Horwood, J. (1997). Breastfeeding reduces the severity of 
neonatal abstinence syndrome. Journal Paediatric Child Health, 33, A38.

Mann, N. P., Haddow, R., Stokes, L, Goodley, S., \& Rutter, N. (1986). Effect of night and day on preterm infants in a newborn nursery: A randomized trial. British Medical Journal, 293, 1265-1267.

Marcellus, L. (2001). Care of substance-exposed infants: The current state of practice in Canadian hospitals. Journal of Perinatal and Neonatal Nursing, 16(3), 51-68.

Marcellus, L. (2007). Neonatal abstinence syndrome: Reconstructing the evidence. Neonatal Network, 26(1), 33-40.

McConnell-Henry, T., Chapman, Y., \& Francis, K. (2011). Member checking and Heideggerian phenomenology: A redundant component. Nurse Researcher, 18(2), 28-37.

McEwen, M., \& Wills, E. M. (2011). Theoretical basis for nursing. Philadelphia, PA: Lippincott Williams\& Wilkins.

McQueen, K. A., Murphy-Oikonen, J., Gerlach, K. \& Montelpare, W. (2011). The impact of infant feeding method on neonatal abstinence scores of methadone-exposed infants. Advances in Neonatal Care, 11(4), 282-290.

Mefford, L. C., \& Alligood, M. R. (2011). Evaluating nurse staffing patterns and neonatal intensive care unit outcomes using Levine’s conservation model of nursing. Journal of Nursing Management, 19, 998-1011.

Mercer, R. T. (2004). Becoming a mother versus maternal role attainment. Journal of Nursing Scholarship, 36(3), 226-232.

Miles, M. B., \& Huberman, A.M. (1994). Qualitative data analysis: A sourcebook of new methods (2nd ed.). Thousand Oaks, CA: Sage.

Miller, C. L., White, R., Whitman, T. L., O’Callaghan, M. F., \& Maxwell, S. E. (1995). The 
effects of cycled versus noncycled lighting on growth and development in preterm infants. Infant Behavioral Development, 18, 87-95.

Morgan, J., \& Knox, J. (1987). Characteristics of "best” and "worst” clinical teachers as perceived by university nursing faculty and students. Journal of Advanced Nursing, 12(3), 331-337.

Morse, J. M. (1991). Qualitative nursing research: A contemporary dialogue (Revised ed.). Newbury Park, CA: Sage.

Morse, J. M. (1994). Emerging from the data: The cognitive process of analysis in qualitative inquiry. In J. M. Morse (Ed.). Critical issues in qualitative research methods. Newbury Park, CA: Sage.

Murphy-Oikonen, J., Brownlee, K., Montelpare, W., \& Gerlach, K. (2010). The experiences of NICU nurses in caring for infants with Neonatal Abstinence Syndrome. Neonatal Network, 29(5), 307-313.

Murphy-Oikonen, J., Montelpare, W. J., Bertoldo, L, Southon, S., \& Persichino, N. (2012). The impact of a clinical practice guideline on infants with neonatal abstinence syndrome. British Journal of Midwifery, 20(7), 493-501.

National Association of Neonatal Nurses. (2009). Instructions for writing clinical practice guidelines for the National Association of Neonatal Nurses. Retrieved October, 2013 from http://www.nann.org/uploads/files/Instructions_for_Writing_Clinical_Practice_Guideline s.pdf

National Institute of Health. (2012). Start4life: Guide to bottle feeding. Retrieved from http://www.nhs.uk/start4life/documents/pdfs/start4life_guide_to_bottle_feeding.pdf 
Neo Advances. (2013). Assessing signs and symptoms of neonatal abstinence using the Finnegan Scoring Tool, an inter-observer reliability program. Retrieved October, 2013 from http://neoadvances.com

Nelson, M. M. (2013). Neonatal Abstinence Syndrome: The nurse’s role. International Journal of Childbirth Education, 28(1), 38-42.

Nehring, V. (1990). Nursing clinical teacher effectiveness inventory: A replication study of the characteristics of 'best' and 'worst' clinical teachers as perceived by nursing faculty and students. Journal of Advanced Nursing, 15(8), 934-940.

O’Brien, C. M., \& Jeffrey, H. E. (2002). Sleep deprivation, disorganization and fragmentation during opiate withdrawal in newborns. Neonatal Intensive Care, 15(4), 27-32.

Oie, J., \& Lui, K. (2007). Management of the newborn infant affected by maternal opiates and other drugs of dependency. Journal of Paediatrics and Child Health, 43, 9-18.

Ohgi, S. Akiyama, T., Arisawa, K., \& Shigemori, K. (2004). Randomized controlled trial of swaddling versus massage in the management of excessive crying in infants with cerebral injuries. Archives of Disease in Childhood, 89, 212-216.

Oro, A.S., \& Dixon, S.D. (1988). Waterbed care of narcotic-exposed neonates. A useful adjunct to supportive care. The Cochrane Controlled Trials Register.

Ostrea, E. M., Chavez, C. J., \& Strauss, M. E. (1976). A study of factors that influence the severity of neonatal narcotic withdrawal. Journal of Pediatrics, 88(4 pt. 1), 642-645.

Patrick, S. W., Schumacher, R. E., Benneyworth, B. D.,Krans, E. E., McAllister, J. M., \& Davis, M. M. (2012). Neonatal Abstinence Syndrome and associated health care expenditures: United States, 2000-2009. Journal of the American Medical Association (JAMA), 307(18), 1934-1940. 
Penz, K. L., \& Bassendowski, S. L. (2006). Evidence-based nursing in clinical practice: Implications for nurse educators. Journal of Continuing Education in Nursing, 37(6), 250-269.

Perlstein, M. (1947). Congenital morphinism, a rare cause of convulsions in the newborn. Journal of the American Medical Association, 135, 633.

Peters, K. L. (2009). Infant handling in the NICU: Does developmental care make a difference? An evaluative review of the literature. Journal of Perinatology, 13(3), 83-109.

Pinto, F, Torrioli, M., Tempesta, E., \& Fundaro, C. (1998). Sleep in babies born to chronically heroin addicted mothers: A follow up study. Drug and Alcohol Dependence, 21, 43-47.

Pritham, U. A., Troese, M., \& Stetson, A. (2007). Methadone and buprenorphine treatment during pregnancy: What are the effects on infants? Nursing for Women's Health 11(6), 558-567.

Pritham, U. A., Paul, J. A., \& Hayes, M. J. (2012). Opioid dependency in pregnancy and length of stay for Neonatal Abstinence Syndrome. Journal of Obstetric, Gynecologic, and Neonatal Nursing (JOGNN), 41,180-190.

Punch, M. (1994). Politics and ethics in qualitative research. In N. K. Denzin \& Y. S. Lincoln (Eds.) Handbook of qualitative research (pp. 86-97). Thousand Oaks, CA: Sage.

Quirk Enterprises. (2014). When working with qualitative research findings, is one more valuable than the other? Retrieved from http://www.quirks.com/articles/a2002/20020609.aspx?searchID=111842881\&sort=9

Rayburn, W. F., \& Bogenschutz, M. P. (2004). Pharmacotherapy for pregnant women with addictions. American Journal of Obstetrics and Gynecology, 191, 1885-1897.

Richards, L., \& Morse J. M. (2007) Readme first for a user's guide to qualitative methods (2nd 
ed.). Thousand Oaks, CA: Sage.

Richardson, H., Walker, A. M., \& Horne, R. S. C. (2010). Influence of swaddling experience on spontaneous arousal patterns and autonomic control in sleeping infants. Pediatrics, 57(1), 85-91.

Rivkees, S. A. (2004). Emergence and influences of circadian rhythmicity in infants. Clinical Perinatology, 31, 217-228.

Roper, J. M., \& Shapira, J. (2000). Ethnography in nursing research. Thousand Oaks, CA: Sage.

Rosen, T. S., \& Pippenger, C. E. (1975). Disposition of methadone and its relationship to severity of withdrawal in the newborn. Addictive Diseases: An International Journal, 2(1), 168-177.

Rosen, L. A. (2008). Infant sleep and feeding. Journal of Gynecologic and Neonatal Nursing, 37, 706-714.

Sarkar, S., \& Donn, S. M. (2006). Management of neonatal abstinence syndrome in neonatal intensive care units: A national survey. Journal of Perinatology, 26, 15-17.

Schore, A. N. (2001). The effects of early relational trauma on right brain development, affect regulation, and infant mental health. Infant Mental Health Journal, 22(1-2), 201-269.

Seligman, N. S., Salva, N., Hayes, E. J., Dysart, K. C., Pequignot, E. C., \& Baxter, J. K. (2008). Predicting length of treatment for neonatal abstinence syndrome in methadone-exposed neonates. [Department of Obstetrics and Gynecology Faculty Papers, Paper 11]. 
Retrieved September, 2013 from http://jdc.jefferson.edu/obgynfp/11

Sharp HealthCare. (2007). The sharp experience. Retrieved March, 2014 from http://www.sharp.com/search/index.cfm?lang=en\&q=culture+of+care

Slatore, C. G., Hansen, L. Ganzini, L., Press, N. Osborne, M. L., Chesnutt, M. S., \& Mularski, R. A. (2012). Communication by nurses in the intensive care unit: Qualitative analysis of domains of patient-centered care. American Journal of Critical Care, 21(6), 410-418.

Spradley, J. P. (1980). Participant observation. New York, NY: Holt, Rinehart and Winston.

Strauss, M. E., Lessen-Firestone, J. K., Starr, R. H., \& Ostrea, E. M. (1975). Behavior of narcotics-addicted newborns. Child Development, 46(4), 887-893.

Streubert, J. J., \& Carpenter, D. R. (2011). Qualitative research in nursing: Advancing the humanistic imperative (5th ed.). Philadelphia, PA: Lippincott, Williams \& Wilkins.

Substance Abuse and Mental Health Services Administration. (2011). Results from the 2010 national survey on drug use and health: National findings. Rockville, MD: Office of Applied Studies, NSDUH. Retrieved September, 2013 from http://www.whitehouse.gov/sites/default/files/ondcp/policy-andresearch/nsduhresults2011.

The AGREE Collaboration. (2003). Development and validation of an international appraisal instrument for assessing the quality of clinical practice guidelines: The AGREE project. Quality and Safety in Health Care, 12, 18-23.

The Brazelton Institute. (2012). New neonatal behavioral assessment scale training program. Retrieved September, 2013 from http://www.brazelton-institute.com/train.html Tikotzky, L., De Marcas, G., Har-Toov, J., Dollberg, S., Bar-Haim, Y., \& Sadeh, A. (2009). Sleep and physical growth in infants during the first 6 months. Journal of Sleep Research, 
19(1), 103-110.

Utriainen, K., \& Kyngäs, H. (2009). Hospital nurses’ job satisfaction: A literature review. Journal of Nursing Management, 17(8), 1002-1010.

Vandenberg, K. A. (2007). State systems development in high-risk newborns in the neonatal intensive care unit: Identification and management of sleep, alertness, and crying. Journal of Perinatal and Neonatal Nursing, 21(2), 130-139.

van Dijk, M., Peters, J. W. B., van Deventer, P., \& Tibboel, D. (2005). The COMFORT behavior scale: A tool for assessing pain and sedation in infants. American Journal of Nursing, 105(1), 33-36.

Van Sleuwen, B.E., Engelberts, A.C., Boere-Boonekamp, M.M., Kuis, W., Schulpen, T.W., \& L'Hoir, M.P. (2007). Swaddling: A systematic review. Pediatrics, 120 (4), e1097-106.

Velez, M., Jansson, L., Svikis, D., Jones, H., Walters, V., \& Chilcoat, H. (2001). Physical, sexual and emotional abuse in pregnant substance dependent women. Drug and Alcohol Dependence, 63(supp. 1), S163.

Velez, M., \& Jansson, L. M. (2008). The opioid dependent mother and newborn dyad: Nonpharmacologic care. Journal of Addiction Medicine, 2(3), 113-120.

Watson, J. (1979). Nursing: The philosophy and science of caring. Boston, MA: Little \& Brown.

Watson, J. (1985a). Nursing: Human science and human care-A theory of nursing. Norwalk, CT: Appleton-Century-Crofts.

Watson, J. (1988). Nursing: Human science and human care-A theory of nursing. New York, NY: National League for Nursing.

Werner, .O., \& Schoepfle, G. M. (1987). Systematic fieldwork: Foundations of ethnography 
and interviewing. Newbury Park, CA: Sage.

White, R. (2011). The newborn intensive care unit environment of care: How we got here, where we're headed, and why. Seminars in Perinatology: Journal of Perinatology, 29, 623-629.

Whittemore, R. (2005). Combining evidence in nursing research: Methods and implications. Nursing Research, 54(1), 56-62.

Wilson, G. S. (1975). Somatic growth effects of perinatal addiction. Addictive Diseases, 2(2), 333-345.

Wilson, V. J., McCormack, B. G., \& Ives, G. (2005). Understanding the workplace culture of a special care nursery. Journal of Advanced Nursing 50(1), 27-38.

Wolcott, H. F. (1999). Ethnography: A way of seeing. Walnut Creek, CA: AltaMira Press.

Woods, P. (1992). Successful writing for qualitative researchers (2nd ed.). New York, NY: Routledge, Taylor and Farmer Group.

World Health Organization (1999). The ten steps to successful breastfeeding, protecting, promoting and supporting breast-feeding: The special role of maternity services. Retrieved October, 2013 from https://www.babyfriendlyusa.org/about-us/baby-friendlyhospital-initiative/the-ten-steps

World Health Organization. (2012). Growth charts. Retrieved October, 2013 from http://www.cdc.gov/growthcharts/who_charts.htm 


\section{APPENDICES}

Appendix A

Empirical Research on Nonpharmacologic Nursing Interventions

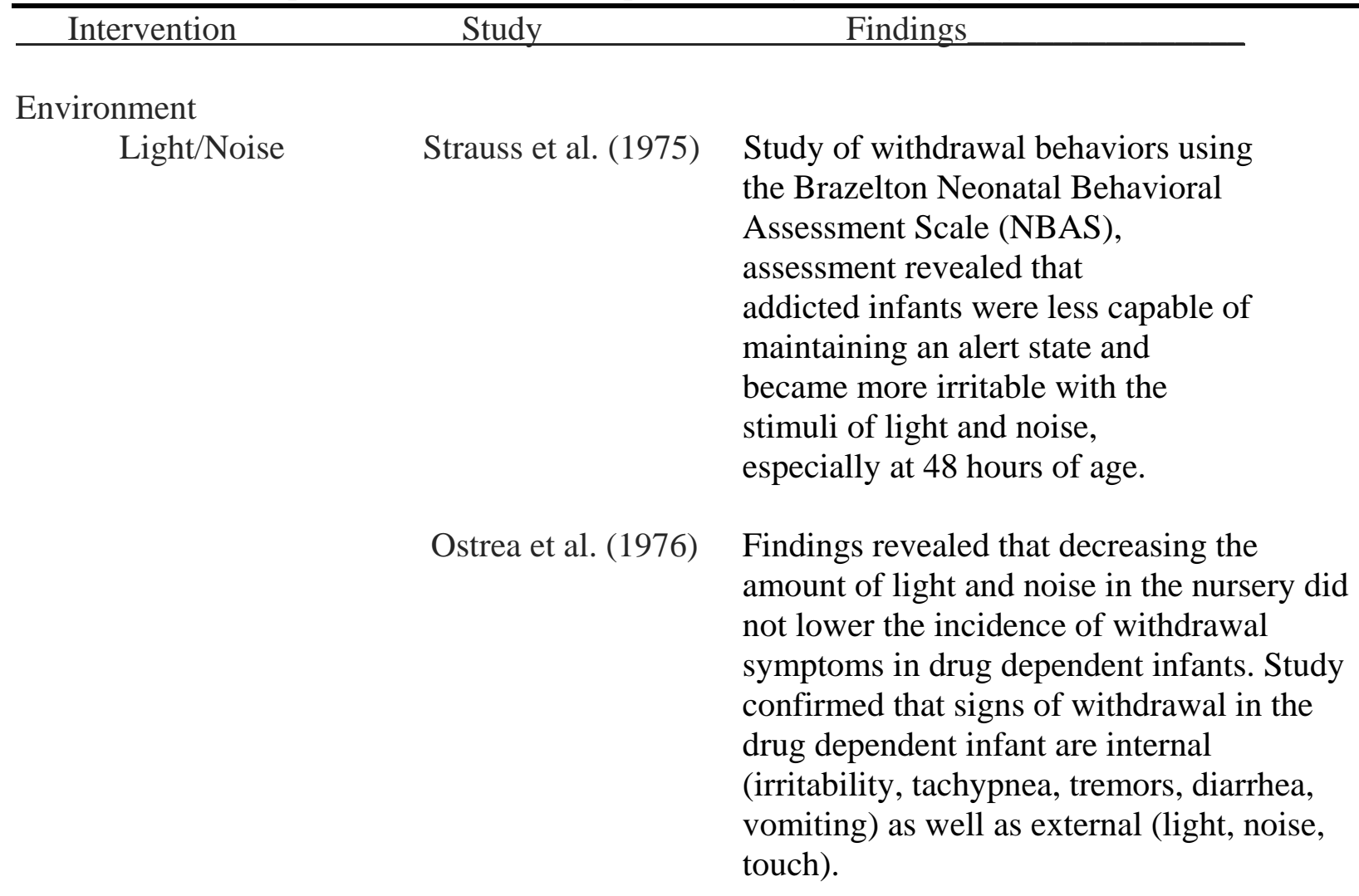

Touch None

Rest and Sleep

$\begin{array}{ll}\begin{array}{l}\text { Swaddling } \\ \begin{array}{l}\text { Non-Nutritive } \\ \text { Sucking }\end{array}\end{array} & \text { None } \\ \text { Rocking } & \text { Oro \& Dixon (1988) } \begin{array}{l}\text { Lower CNS scores permitted the } \\ \text { infants on waterbeds to require less } \\ \text { pharmacology to decrease symptoms } \\ \text { and assisted with earlier weight gain } \\ (\mathrm{n}=30) .\end{array}\end{array}$


D’Apolito (1999)

Positioning

Maichuk et al.(1999)

Ballard (2001)

Breast milk/

Formula
Research showed that when infants' withdrawal symptoms were recorded using mechanical rocking beds versus standard beds, the rocking bed use did not decrease withdrawal symptoms, and actually resulted in overstimulation of the infants $(n=14)$.

Positioning of NAS infants found that swaddled prone lying infants $(n=25)$ proved to have decreased withdrawal symptoms over swaddled supine lying infants $(n=23)$. Although prone lying infants experience less crying and have decreased energy requirements, current standards to prevent SIDS require that all infants sleep supine for safe sleep.

Breast fed infants exhibit decreased withdrawal symptoms and decreased LOS ( $n=6)$. Finnegan score used to assess infants.

Abdel-Latif et al. (2006) Finnegan scores were significantly lower in the breast milk group over the formula fed group (after stratifying for prematurity and polydrug and methadone exposure. Significantly fewer infants required weaning with oral morphine and the median time for withdrawal occurred later in the breast milk group $(n=190)$. Finnegan score used to assess infants.

Jansson, et al. (2008) Fewer infants required pharmacology for NAS, not statistically significant (4 infants compared with 1 breastfed infant). Nine neurobehavioral outcomes used to assess infants. 
Dryden, et al. (2009)

Gerlach (2010)

McQueen et al. (2011)

Isemann et al. (2011)

Pritham et al. (2012)
Breastfeeding $>72$ hours was associated with halving the odds of the infant receiving pharmacotherapy for NAS ( $\mathrm{n}=450$ mothers and infants). Lipsitz scoring tool used to assess infants.

Retrospective cohort study in Canada measured methadone exposed infants (n breast $=8$, $\mathrm{n}$ formula $=9$, $\mathrm{n}$ combination $=$ 11) and found that the infants who were breastfed exhibited less overall neonatal abstinence symptoms when looking at the magnitude, duration and area of scores when compared with infants who were fed formula alone or a combination of breast milk and formula. Finnegan score used to assess infants.

Infants predominantly breastfed had significantly lower NAS scores, suggesting decreased severity and duration of withdrawal symptoms when compared with infants who were fed a combination of breast milk and formula or formula alone ((n breast $=8, \mathrm{n}$ combination $=11, \mathrm{n}$ formula $=$ 9). Modified Finnegan scoring tool used to assess infants.

Breast feeding is associated with a shorter median duration of pharmacological treatment in preterm and term infants and decreased LOS when compared to formula fed infants (n breast $=56$, $n$ formula $=56$ ). Finnegan score used to assess infants.

LOS was shorter in breastfed infants than formula-fed infants or infants who received formula and breast milk. Neonates with prenatal exposure to methadone spent more days in the hospital (21 versus 14 days) for treatment of NAS than those prenatally exposed to buprenorphine. (MMT =136, BMT = 16; LOS was used to assess infants. 
Finnegan Scoring

By Nurses

Lucas \& Knobel (2012) Evidence-based clinical guidelines and Finnegan scoring equip caregivers with the necessary tools to consistently and accurately assess an infant with NAS when using the FNAST (DVD) educational project. $\mathrm{N}=10$.

Nurses as Caregivers

Fraser et al. (2007) interpretive methods by group interviews involving eight nurses. Many nurses felt that their care of the infant had to be balanced between treatments for the NAS infant with time spent in consoling the infant. Questions rose as to the optimal environment for NAS infant care; the NICU setting or separate rooms for the infant and mother to promote attachment.

Murphy-Oikonen et al. Nurses experience expectations (2010)

to provide maternal care to infants. Providing maternal care can be in conflict with their role as highly technically trained NICU nurses. Qualitative study with fourteen nurses using open-ended, computer assisted interviews. 


\section{Appendix B}

\section{Healthcare Facility IRB}

\section{PAichitren's
Hospital}

INSTITUTIONAL. REVIEW BOARD

April 7, 2014

Monica M. Nelson

1905 Emoriland Blvd

Knoxville, TN 37931

RE: Expedited approval of ETCH \#214: Nurses Culture of Care with NAS Infants: A Focused

Ethnography

Dear Ms. Nelson:

As Coordinator of the East Tennessee Children's Hospital (ETCH) Institutional Review Board (IRB), under the direction of the IRB Chairman, I have reviewed the protocol application identified above through the expedited review procedure. I have determined that the study poses minimal risk to participants and the application is approved through the expedited process under 45 CFR $46.110(\mathrm{~b})$. This approval is contingent upon approval by the East Tennessee State University IRB. Once their approval is granted, please forward a copy of the approval letter and a final copy of consent forms to be stamped by the ETCH IRB for use with participants.

If there are any problems with the study or changes to the study design that relate to human participants, please notify the East Tennessee Children's Hospital Institutional Review Board as soon as possible.

For your calendar, the expiration date of this approval is April 6, 2015. Continuing review is scheduled for March 2015. At that time, a continuing review form will be sent to you to fill-out and return to the IRB for annual review. If you complete the study prior to this date, please forward a summary of the results and closure notice. The expedited approval will be reported to the full, convened IRB in May 2014.

If you have further questions or need assistance, please contact me at (865) 541-8411 or Jacque Van Audenhove at (865) 541-8290.

Sincerely,

Tauce Temp

Nancy Camp, RN, MN, CPNP

IRB Coordinator

2018 Clinch Ave

Knoxville, TN 37916

jaudenhove@etch.com

p. 865.541 .8290 


\title{
Appendix C:
}

\section{Healthcare Facility Research Council Approval Letter}

BBSW1 132,3013

\begin{abstract}
To the best of the fenow ledge and ability of the investigator(s), the information provided in

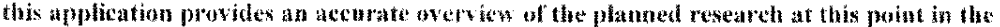
deselopanent of the research proposat. Becanse this Researeh Council review of the monosal oecurs while the proposal is still in the developmental phases, it is acknowledped by both the investigators and the Rexeateh Council that the preposal may andergo some minor revisions before timal anproval by the Institutional Review Board. The avestgator(x) isfare requested to notify the Research Conncil at any major protocol revisions.
\end{abstract}

The investgaters acknowledge their thderstanding that this resested camot be tondeded until this project is also reviewed and approved by the lastitational keview board of Fast Tennessec Children's Hospital.

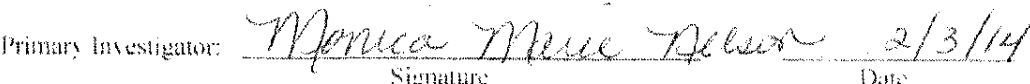
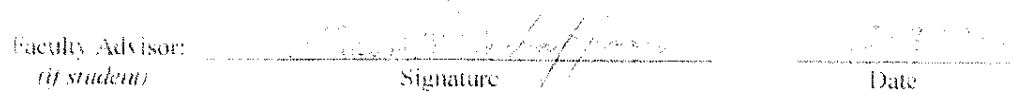

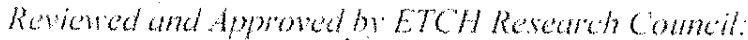

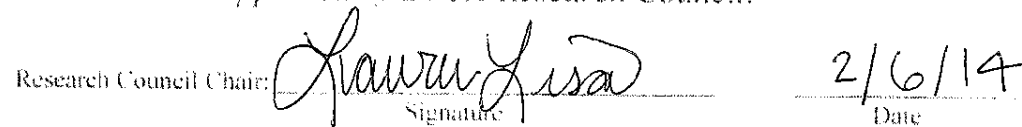


Appendix D

ETSU Informed Consent Letter for Participants-Participant Observation

\section{EAST TENNESSEE STATE UNIVERSITY INFORMED CONSENT DOCUMENT Observational Phase}

PRINCIPAL INVESTIGATORS: Monica Marie Nelson, RN, M. Ed., PhD(c), Doctoral student Dissertation Advisor: Dr. Masoud Ghaffari

TITLE OF PROJECT: Nurses Culture of Care with Neonatal Abstinence Syndrome (NAS) Infants: A Focused Ethnography

This informed consent will explain about being a research participant in a study. It is important that you read this material carefully and then decide if you wish to be a volunteer.

\section{Purpose}

This study is sponsored by East Tennessee State University (ETSU), College of Nursing, Johnson City, TN 37614 and is being directed by Monica Nelson and Dr. Ghaffari. NAS infants are increasingly being cared for in the inpatient hospital setting by NICU nurses. The purpose of this ethnographic study is to explore and describe the culture of care for infants with NAS provided by NICU 3 nurses at East Tennessee Children's Hospital.

\section{Procedures and Study Duration}

Participant Observation: If you agree to be a part of this study, there will be a period of 5 weeks where the PI will be observing you on the NICU 3 unit. During this time, you may be asked "how" or "why" you perform a nursing task with an infant. Observing you will allow the researcher to see day-to-day activities of the nurses and see real world examples of the culture of care of nurses who care for infants with NAS.

After the observation period is complete, you may be asked to additionally participate in an interview. If you are eligible to be part of the interview phase of this project, the researcher will provide you with detailed information about the interview in a different informed consent document.

\section{Alternative Procedure}

None.

\section{Potential Risks and Benefits}


The researcher is not aware of any known risks to you for participating in this study. There is also no anticipated direct benefit to you from this study. We hope that the results of the study may benefit and encourage people who work with infants with NAS.

\section{Contact for Questions}

In the event of research related injuries or if you have further questions about the research, you may contact Monica Nelson at (865)765-6121 or Dr. Masoud Ghaffari at (423) 439-4513. If you have any questions about your rights as research participant you may contact the Chairman of the Institutional Review Board (IRB) at East Tennessee State University at (423)439-6055 or Jacque Van Audenhove at East Tennessee Children's Hospital at (865) 541-8290. If you have any questions or concerns about the research and want to talk to someone independent of the research team or you can't reach the study staff, you may call an IRB Coordinator at 423/4396055 or $423 / 439 / 6002$.

\section{Confidentiality}

Every attempt will be made to see that your study results are kept confidential. A copy of the records from this study will be stored in a locked cabinet in the researcher's home office at 1905 Emoriland Blvd., Knoxville, Tennessee, 37917 for at least 5 years after the end of this research. The results of this study may be published and/or presented at meetings without naming you as a participant. Although your rights and privacy will be maintained, the Institutional Review Board at East Tennessee State University and DHHS have access to the study records. Your records will be kept completely confidential according to the current legal requirements. They will not be revealed unless required by law, or as noted above.

Participation in this research experiment is voluntary. You may refuse to participate. You can also quit at any time. If you quit or refuse to participate, the benefits or treatment to which you are otherwise entitled will not be affected. You may quit by calling Monica Nelson, whose phone number is (865)765-6121. You will be told immediately if any of the results of the study should reasonably be expected to make you change your mind about staying in the study.

By signing below, you confirm that you have read or had this document read to you. You will be given a signed copy of this informed consent document.

Signature of Participant

Date

Signature of Researcher

Date 


\title{
Appendix E
}

\section{ETSU Informed Consent Letter for Participants-Interviews}

\author{
EAST TENNESSEE STATE UNIVERSITY \\ INSTITUTIONAL REVIEW BOARD \\ INFORMED CONSENT DOCUMENT \\ Interview Phase
}

PRINCIPAL INVESTIGATORS: Monica Marie Nelson, RN, M. Ed., PhD(c), Doctoral student Dissertation Advisor: Dr. Masoud Ghaffari

TITLE OF PROJECT: Nurses Culture of Care with Neonatal Abstinence Syndrome (NAS)

Infants: A Focused Ethnography

This informed consent will explain about being a research participant in a study. It is important that you read this material carefully and then decide if you wish to be a volunteer.

\section{Purpose}

This study is sponsored by East Tennessee State University (ETSU), College of Nursing, Johnson City, TN 37614 and is being directed by Monica Nelson and Dr. Ghaffari. NAS infants are increasingly being cared for in the inpatient hospital setting by NICU nurses. The purpose of this ethnographic study is to explore and describe the culture of care for infants with NAS provided by NICU 3 nurses at East Tennessee Children's Hospital.

\section{Procedures and Study Duration}

If you agree to participate in this study, you will be asked to participate in a face-to-face interview at a time mutually agreed between the researcher conducting the study and you. The interview will take about an hour to complete. During this interview the research will ask a series of questions regarding the culture of care of infants with NAS in the NICU 3 unit. The researcher will audio record the interview and will also take notes. There will be no cost to you from participation in the research.

Then, as the research begins to look at the results of the study, you will be asked to review the found themes and comment on their accuracy.

\section{Alternative Procedure}

None.

\section{Potential Risks and Benefits}

The researcher is not aware of any known risks to you for participating in this study. Benefits include the opportunity to share your thoughts and feeling about the topic.

\section{Contact for Questions}


In the event of research related injuries or if you have further questions about the research, you may contact Monica Nelson at (865)765-6121 or Dr. Masoud Ghaffari at (423) 439-4513. If you have any questions about your rights as research participant you may contact the Chairman of the Institutional Review Board (IRB) at East Tennessee State University at (423)439-6055 or Jacque Van Audenhove at East Tennessee Children's Hospital at (865) 541-8000. If you have any questions or concerns about the research and want to talk to someone independent of the research team or you can't reach the study staff, you may call an IRB Coordinator at 423/439-6055 or 423/439/6002.

\section{Confidentiality}

Every attempt will be made to see that your study results are kept confidential. A copy of the records from this study will be stored in a locked cabinet in the researcher's home office at 1905 Emoriland Blvd., Knoxville, Tennessee, 37917 for at least 5 years after the end of this research. The results of this study may be published and/or presented at meetings without naming you as a participant. Although your rights and privacy will be maintained, the Institutional Review Board at East Tennessee State University and DHHS have access to the study records. Your records will be kept completely confidential according to the current legal requirements. They will not be revealed unless required by law, or as noted above.

Participation in this research experiment is voluntary. You may refuse to participate. You can also quit at any time. If you quit or refuse to participate, the benefits or treatment to which you are otherwise entitled will not be affected. You may quit by calling Monica Nelson, whose phone number is (865)765-6121. You will be told immediately if any of the results of the study should reasonably be expected to make you change your mind about staying in the study.

By signing below, you confirm that you have read or had this document read to you. You will be given a signed copy of this informed consent document.

Signature of Participant

Date

Signature of Researcher

Date

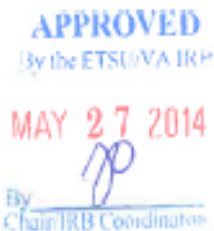

DOCUMENT VERSION EXPIRES

MAY 262015

ITSUIVA IRT 
Appendix F

NICU 3 Research Flyer

\section{Nursing Research: Understanding of the culture of care of the nurses in NICU 3 who care for infants with NAS. ***All participation is voluntary.}

\section{How:}

- Observation on the NICU 3 unit

If you choose to be a part of participate observation, I will be observing how you perform nursing interventions for infants with NAS.

- Face to Face, Individual Interviews

If you choose to participate, I will have one-on-one interviews to gain a deeper understanding of how and why of the interventions employed.

Eligibility: The "core" team of nurses who work on NICU 3 and daily care for infants with NAS.

Inclusion criteria: require that each nurse be a NICU staff nurse, provide direct patient care and predominantly work in NICU 3.

Exclusion criteria: there will be no observation of NICU nurses who do not consistently work on NICU 3 or who are floating to the unit from NICU 1 or NICU 2.

By Whom: Monica Nelson, a doctoral student from East Tennessee State University.

Feel free to contact Monica with any questions about this qualitative research:

-monicamarienelson@gmail.com $-865.765 .6121$ 
***This research has approval from Lori Smith, Sheri Smith, IRB from ETCH, the ETCH Research Council, and IRB from ETSU.

Appendix G

Map of NICU 3

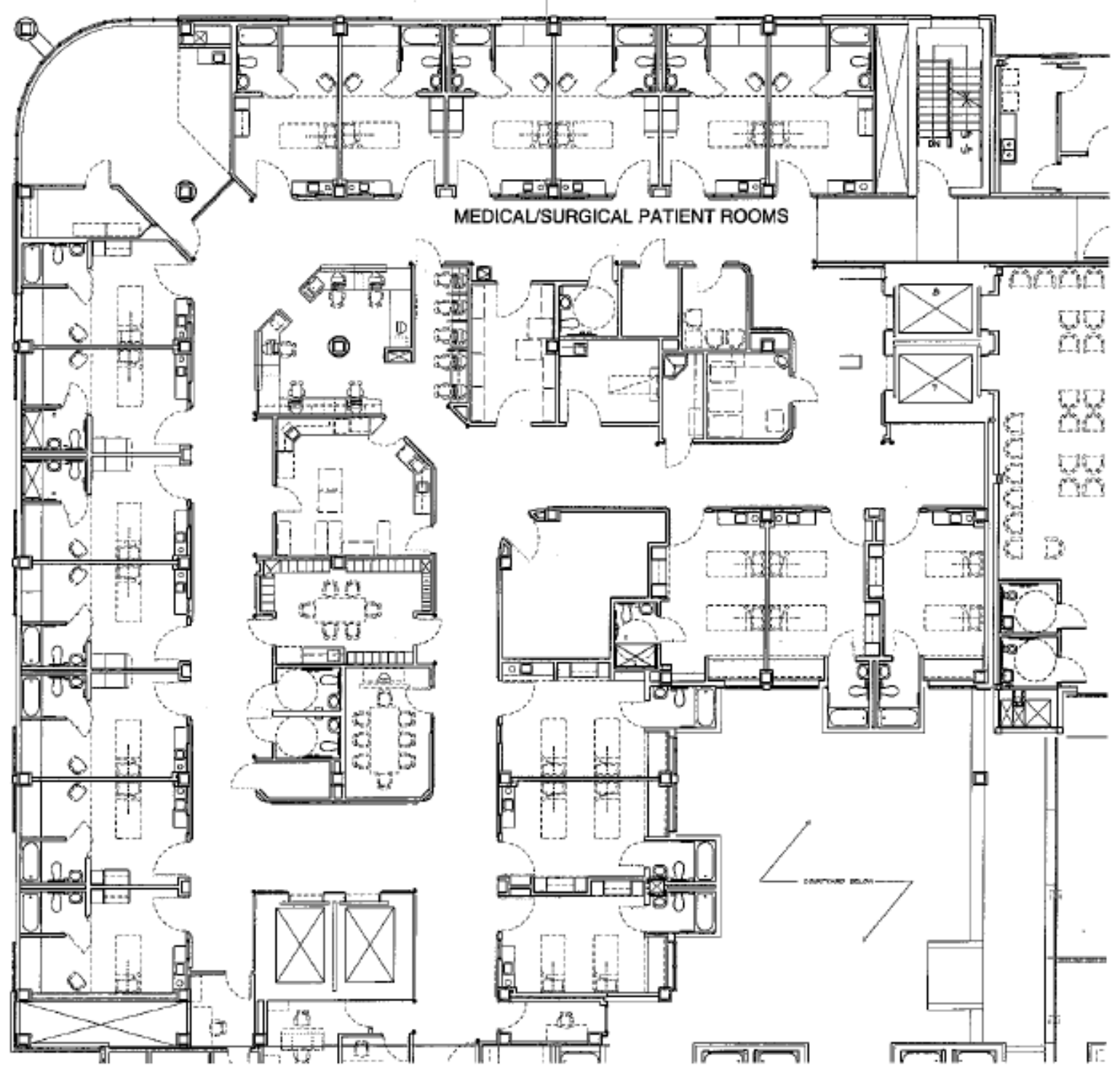




\section{Appendix $\mathrm{H}$}

Interview Guide

Beginning statements: Hello and thank you for consenting to participate in an interview about the culture of care of NICU 3. (Go over informed consent and have participant sign. Answer any questions).

1. Can you tell me what led you down the path of a career in the NICU?

2. Could you please explain how you came to be a part of the "core team" of nurses who work in NICU 3?

3. Tell me what it is like caring for infants with NAS? ... (go with whatever they tell you, emotional strain, reward, behaviors)

4. So, now that I have an understanding of your feelings about caring for these infants, is there anything else about the care of these infants that you would like to share with me?

5. Tell me about the type of care that you provide for these infants?

6. When you think about caring for these infants, what would you consider as a support in providing the care for these infants?

7. Tell me about what it is like working on a specialized team of nurses and other professionals?

8. Tell me about the work environment that you work in?

9. Are there any barriers to providing care?

10. How do you cope with the difficult days?

11. What are the successes you experience in working with these infants?

12. What are the challenges/hardships to working with these infants?

13. What makes work easier for you?

14. What is it about this unit in particular that makes you want to work here?

15. What do you see that this unit is doing well in regards to the care of these infants?

16. What do you see that this unit is not doing well (or needs to improve on) in regards to the care of these infants?

Thank you for sharing your insights on the NICU environment and the care of these infants. I would like to switch gears for a moment and ask you some more specific questions regarding some of the interventions.

1. Are there types of interventions that you have found helpful in soothing these infants? (Then go into your specific questions).

2. I have noticed that all infants are on a 3 hour feeding schedule, could you please describe how you care for infants every 3 hours... what are the nursing interventions that you do?

3. After this every 3-hour care, what are the nursing strategies you use to help infants with NAS to sleep or be comforted between feedings?

Specific Nursing Intervention Questions:

1. What effect does light, noise and touch have on NAS infants? What do you do to control light, noise and touch? (Environment)

2. Describe how you help an infant with NAS to get adequate sleep and rest? (Adequate rest and sleep) 
3. How would you describe feeding an infant with NAS (formula or breast milk)? (Feeding) 4. How would you describe Finnegan scoring? (Assessment and evaluation using Finnegan scoring)

5. What role do you see yourself in with these infants?” (Nurses like mothers to infants)

What successes do you personally experience?

What hardships do you personally experience?

Is there anything else about your experiences on NICU 3 that you have not had the chance to express that you feel is important? (Be comfortable with silence to give people time to generate their thoughts)

Thank you so much for your time and your answers for this interview. 
Appendix I

Coding Sheets

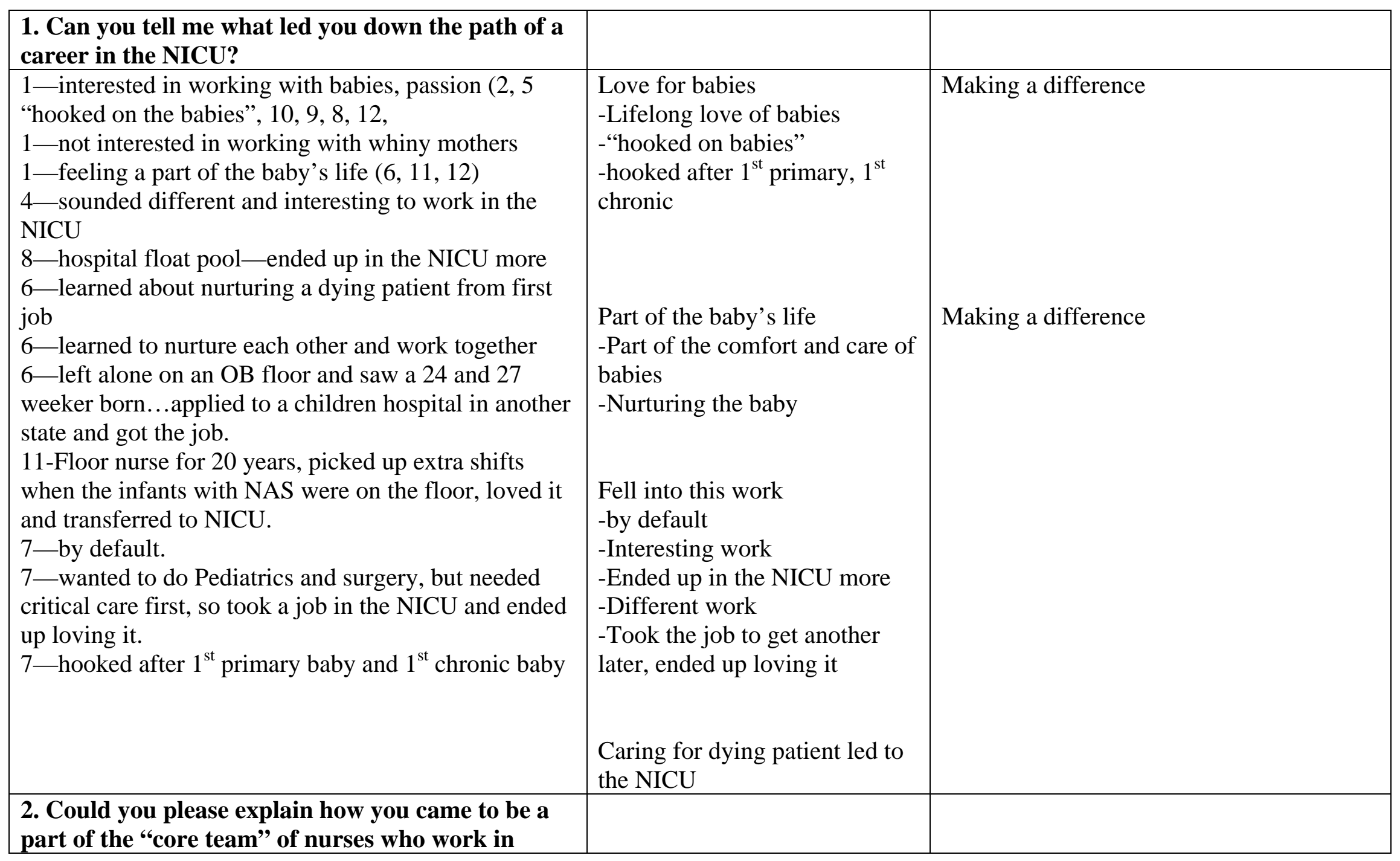




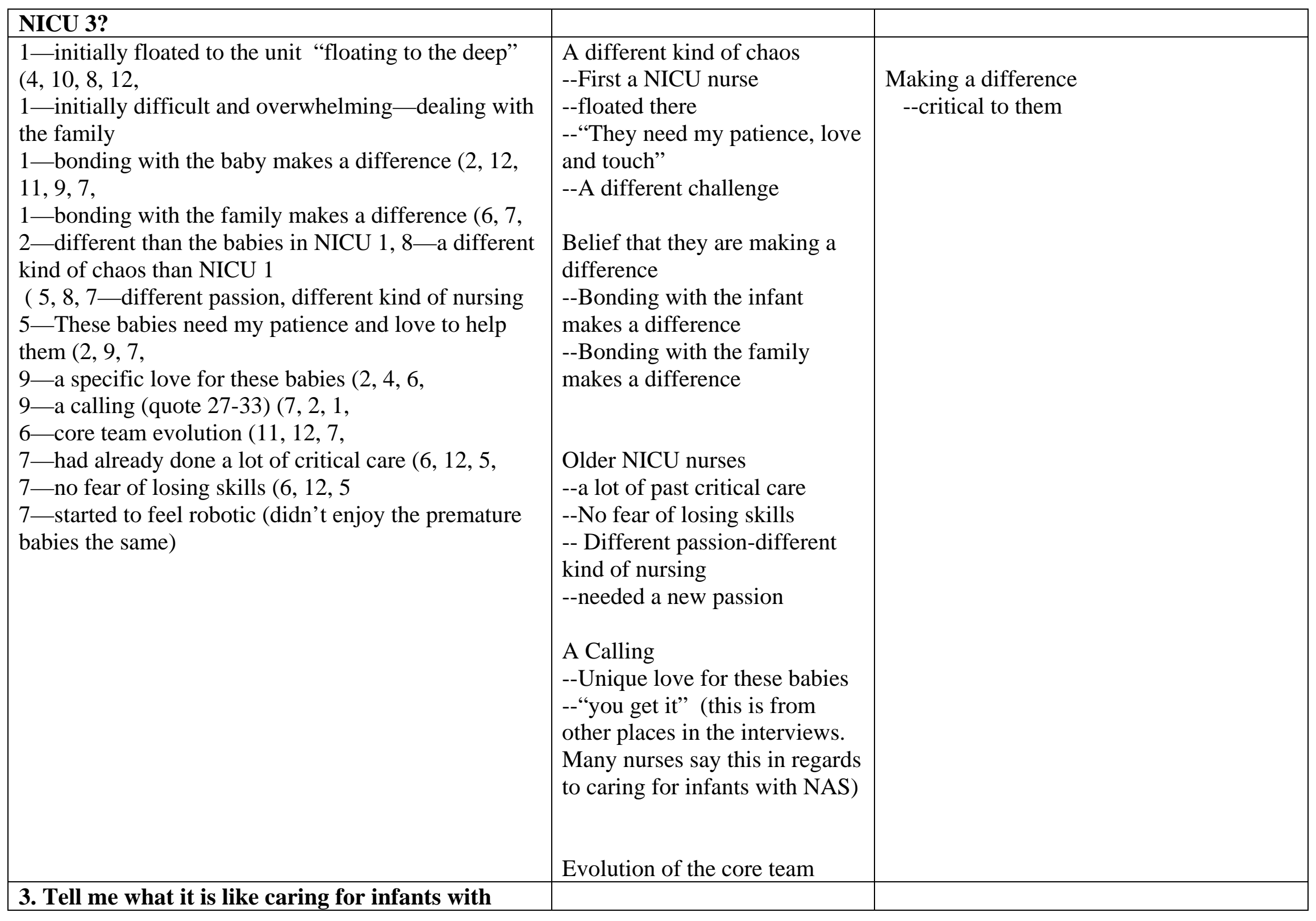




\begin{tabular}{|c|c|c|}
\hline $\begin{array}{l}\text { NAS? ...(go with whatever they tell you--emotional } \\
\text { strain, reward, behaviors) }\end{array}$ & & \\
\hline 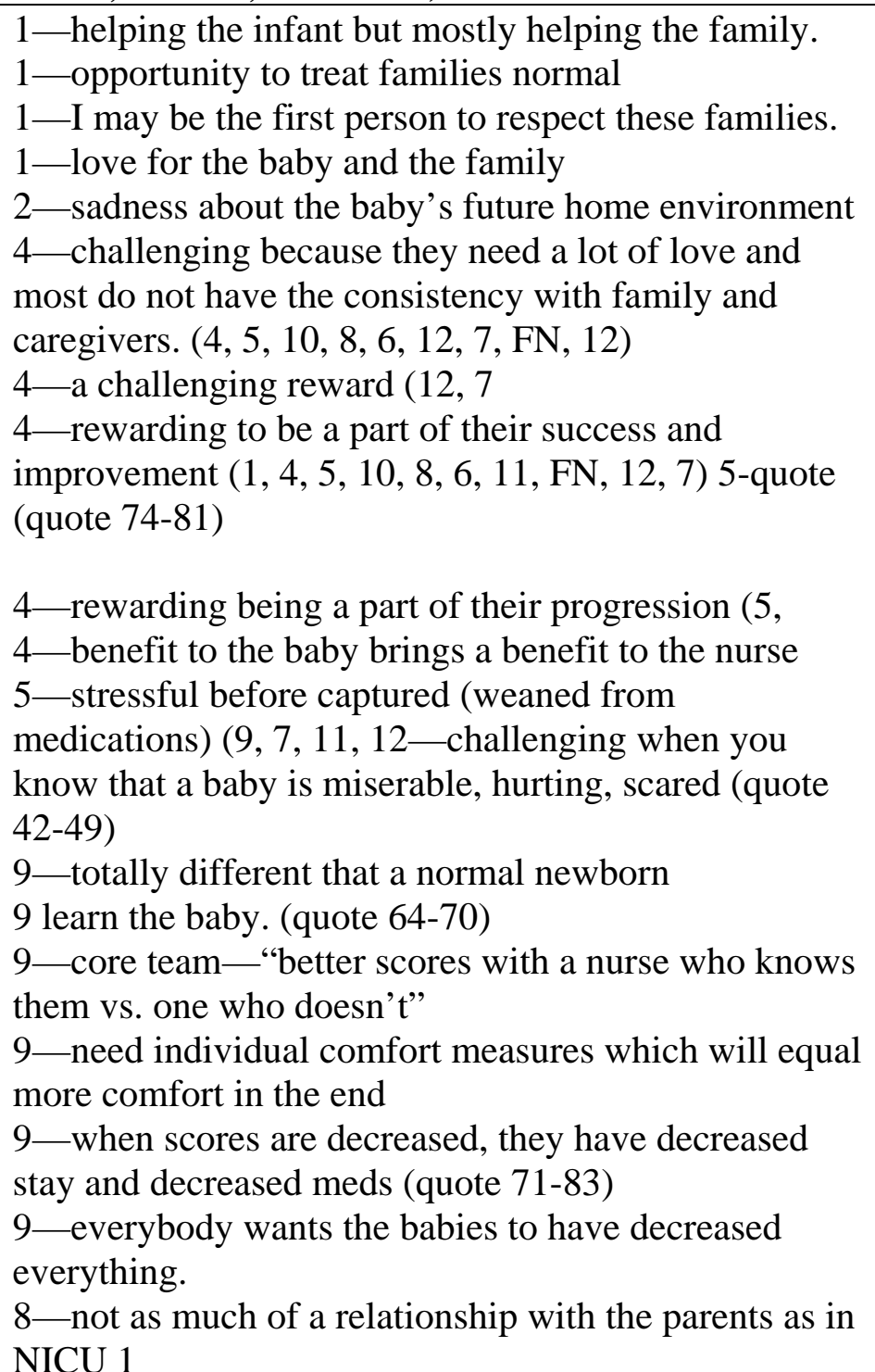 & $\begin{array}{l}\text { Love for families } \\
\text {--Treat families different than } \\
\text { how they are used to being } \\
\text { treated } \\
\text {-- Respect } \\
\text { Challenges } \\
\text {--care of baby } \\
\text {--helping parents } \\
\text {--future for baby } \\
\text { Rewarding } \\
\text {--part of their success } \\
\text {--Benefit to the baby brings } \\
\text { benefit to the nurse } \\
\text {--Attachment of nurse and baby } \\
\text {--Make a difference in the } \\
\text { baby's life } \\
\text { Different kind of care } \\
\text {--unique love } \\
\text {--not normal care } \\
\text {--patience needed } \\
\text {--being there for them }\end{array}$ & $\begin{array}{l}\text { Making a difference } \\
\text {--Wonderful Insanity }\end{array}$ \\
\hline
\end{tabular}




\begin{tabular}{|c|c|c|}
\hline 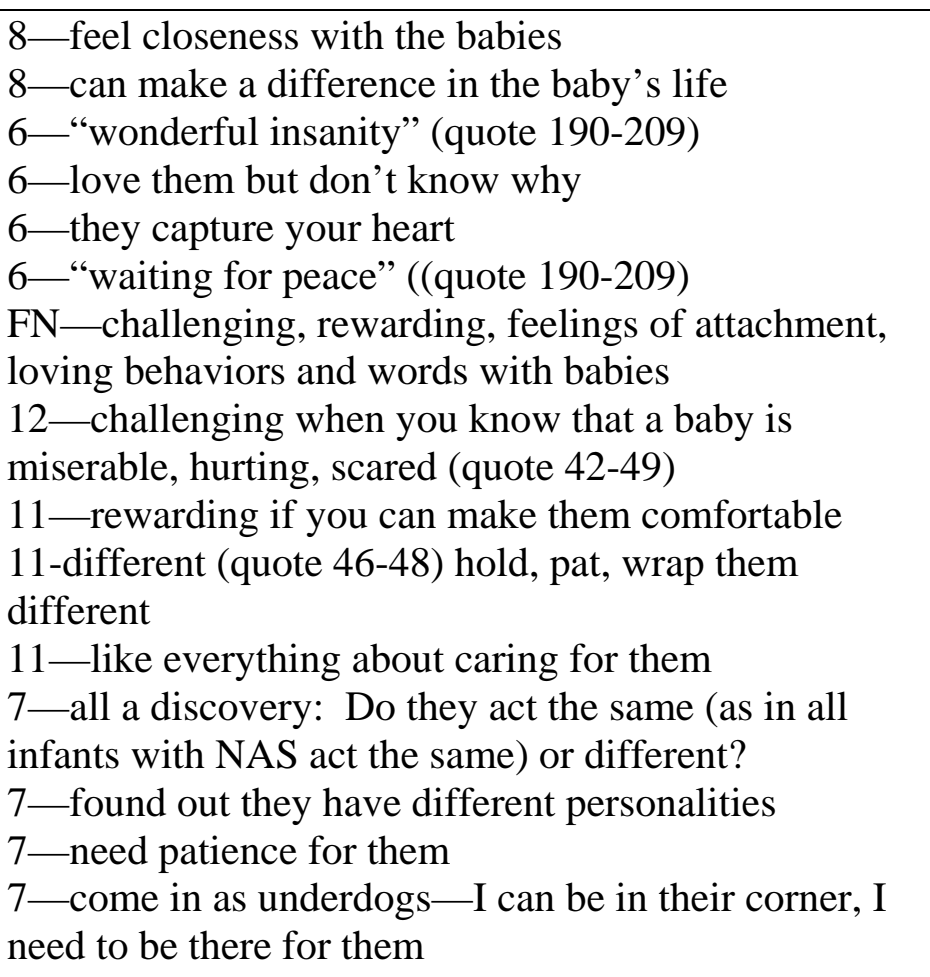 & $\begin{array}{l}\text { Core team knows the babies } \\
\text {--Individual comfort measures } \\
\text {--Intentional care to learn } \\
\text {--Learn their personalities } \\
\text {--Learn the baby } \\
\text {--you just "get it” (phrases used } \\
\text { in many interviews about how } \\
\text { these nurses love these babies. } \\
\text { I get this as well.) }\end{array}$ & "Learn the baby" \\
\hline $\begin{array}{l}\text { 4. So, now that I have an understanding of your } \\
\text { feelings about caring for these infants, is there } \\
\text { anything else about the care of these infants that } \\
\text { you would like to share with me? }\end{array}$ & & \\
\hline $\begin{array}{l}\text { 5-Decisions to come to NICU } 3 \text { from NICU } 1 \text { and } \\
\text { NICU } 2 \text { by NPs: increased doses and family from out } \\
\text { of town can stay with infant in individual room(code?) } \\
\text { 5-PCAs, secretary: all cheering for the babies } \\
\text { 5- you get to know their cries, know the baby } \\
\text { 9- rewarding, challenging } \\
\text { 9-“Can I keep doing this?” (quote 95-101) } \\
\text { 9- try to look at the positive and do my job to the best }\end{array}$ & $\begin{array}{l}\text { How the baby gets to NICU } 3 \\
\text { Everyone cares about the } \\
\text { babies } \\
\text {--caring is hard } \\
\text { Focusing on the positive } \\
\text {-Focus on what the baby gains }\end{array}$ & $\begin{array}{l}\text { Making a difference } \\
\text {--Wonderful insanity } \\
\text { Personal Role Satisfaction }\end{array}$ \\
\hline
\end{tabular}




\begin{tabular}{|c|c|c|}
\hline $\begin{array}{l}\text { of my ability } \\
\text { 9-focus on what the baby gets out of the relationship } \\
\text { as me its nurse } \\
\text { 9-nonjudgmental of parents and their lives-have } \\
\text { not walked in their shoes } \\
\text { 8-you do not get to see the babies very much after } \\
\text { they go home as in NICU } 1 \\
\text { 8-good feeling to see positive outcomes, but wish } \\
\text { she could see more positive (need some positive to } \\
\text { keep you going) }\end{array}$ & $\begin{array}{l}\text { from me } \\
\text {-Desire to see more positive } \\
\text { outcomes } \\
\text { Posture of nurses towards } \\
\text { parents } \\
\text { Lack of future contact with } \\
\text { babies and families }\end{array}$ & $\begin{array}{l}\text { Making a difference } \\
\text { Grief }\end{array}$ \\
\hline \multicolumn{3}{|l|}{$\begin{array}{l}\text { 5. Tell me about the type of care that you provide } \\
\text { for these infants? }\end{array}$} \\
\hline 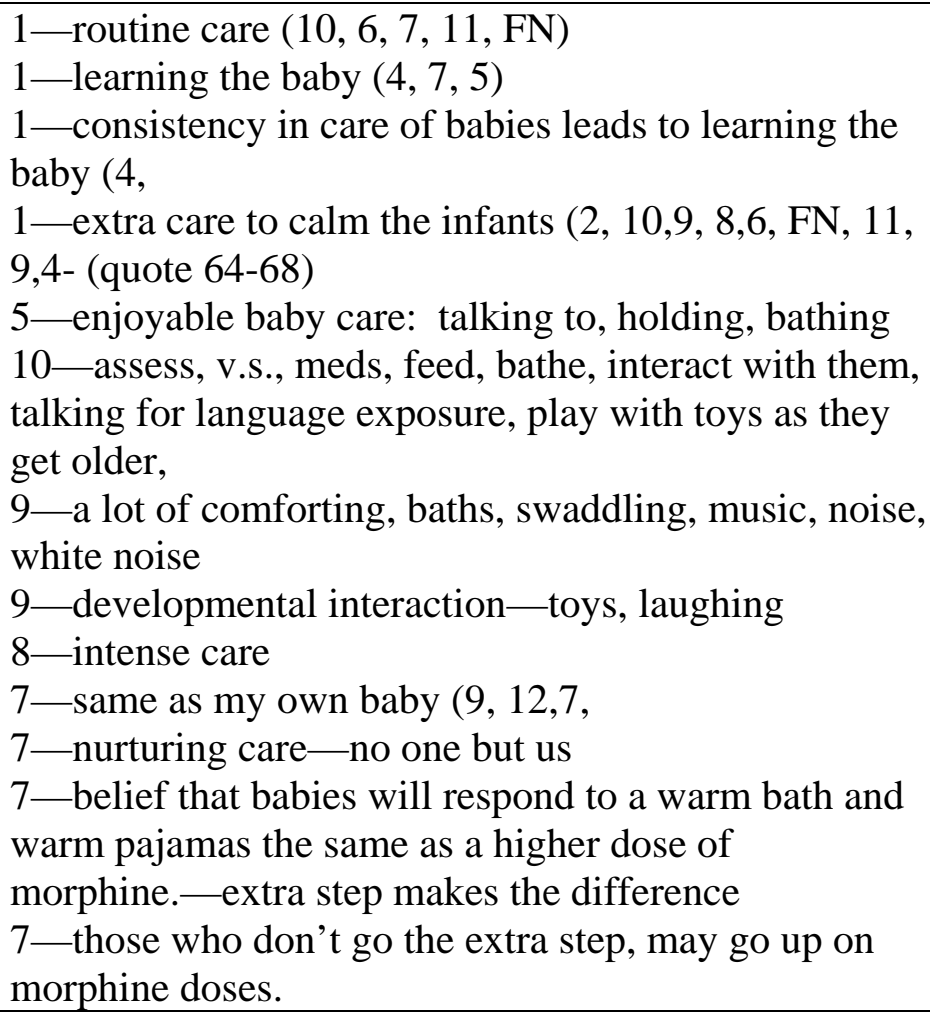 & $\begin{array}{l}\text { Routine care } \\
\text { Belief that extra care counts } \\
\text {--learn the baby } \\
\text {--extra work } \\
\text {-intentional interactions from } \\
\text { the nurse to the baby } \\
\text { Role of the nurse } \\
\text {--as a mother } \\
\text {--nurturer }\end{array}$ & $\begin{array}{l}\text { Personal Role Satisfaction } \\
\text {--nurturer/comforter }\end{array}$ \\
\hline
\end{tabular}




\begin{tabular}{|c|c|c|}
\hline 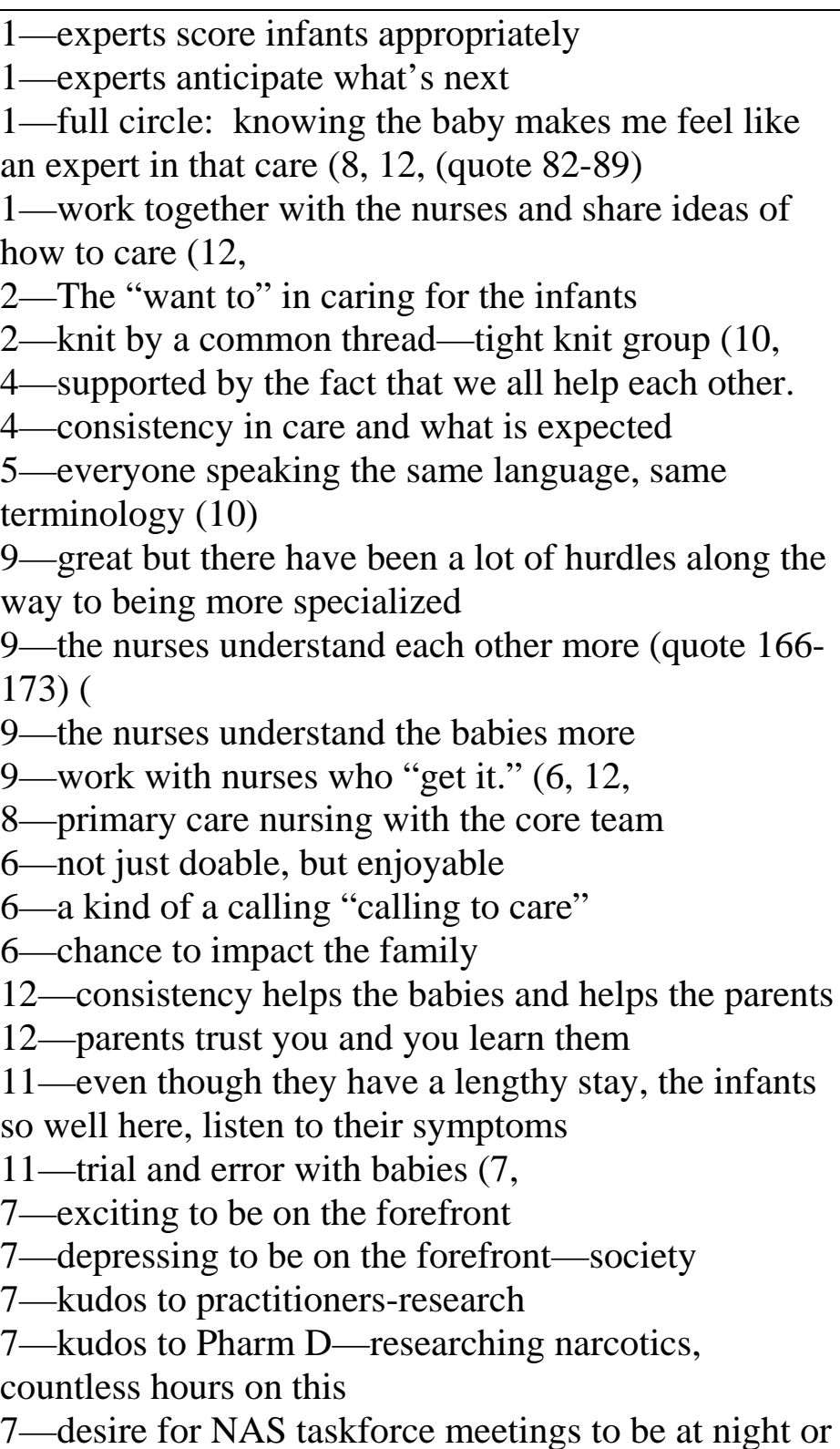 & $\begin{array}{l}\text { Becoming an expert } \\
\text {-Benefit to the baby } \\
\text {-benefit to the parents } \\
\text {-Score appropriately } \\
\text {-Anticipate the baby's needs } \\
\text {-trial and error } \\
\text {-Primary care nursing } \\
\text {-Consistency of care } \\
\text {-learning the baby } \\
\text {-Commitment to keep learning } \\
\text { by all staff }\end{array}$ & $\begin{array}{l}\text { Personal Role Satisfaction } \\
\text {--becoming an expert }\end{array}$ \\
\hline
\end{tabular}




\begin{tabular}{|c|c|c|}
\hline $\begin{array}{l}\text { night-shift accommodating-want to be more a part of } \\
\text { this } \\
\text { 7-they continue to educate themselves } \\
\text { 7-honor to be a part of the "frontier" } \\
\text { 7- proud that other NICUs in the world are learning } \\
\text { from us. }\end{array}$ & & \\
\hline $\begin{array}{l}\text { 8. Tell me about the work environment that you } \\
\text { work in? }\end{array}$ & & \\
\hline 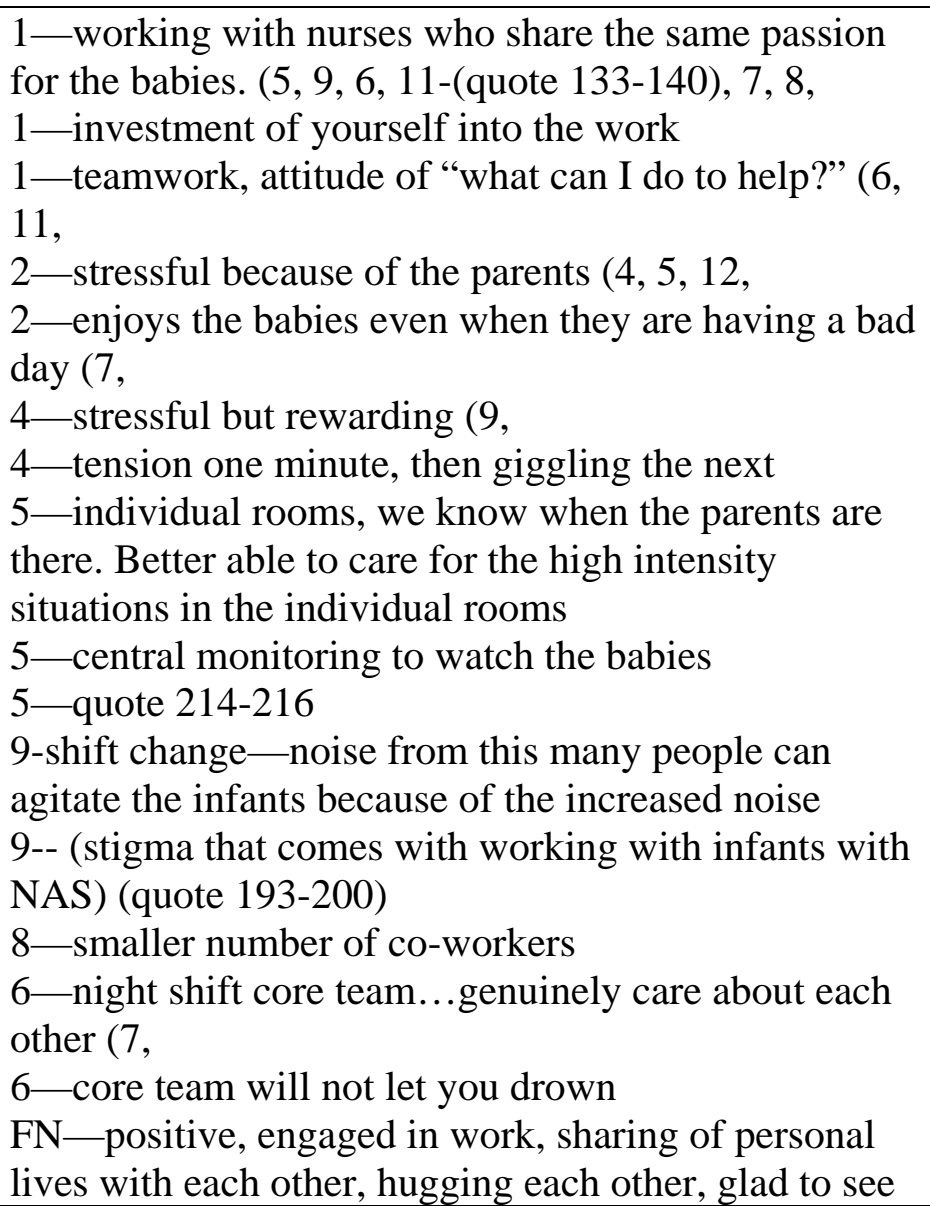 & $\begin{array}{l}\text { Being a part of the Core Team } \\
\text {--same passion } \\
\text {-teamwork } \\
\text {-Know the babies and families } \\
\text {-Some stigma (negative) } \\
\text {-Genuine care for each other } \\
\text { "like a family" } \\
\text {-collaboration of care } \\
\text {-consistency of care } \\
\text {-Sticks together } \\
\text {-Choice to be there } \\
\text {-They have my back and I have } \\
\text { theirs } \\
\text {-Solve problems together-no } \\
\text { nurse alone } \\
\text {-They make all the difference }\end{array}$ & Personal role satisfaction \\
\hline
\end{tabular}




\begin{tabular}{|c|c|c|}
\hline $\begin{array}{l}\text { each other, "like a family", helping each other, joking, } \\
\text { teamwork, helping each other, talking when they are } \\
\text { stressed, checking on each other, consistent in care, } \\
\text { working together, a lot of communication, sharing } \\
\text { what works, collaboration of care, friendly, core team } \\
\text { 12-good overall } \\
\text { 12-frustrating when not enough help } \\
7 \text { - solve problems together, no nurse alone, solve as a } \\
\text { group } \\
7 \text { - celebrate successes } \\
7 \text {-other NICU nurses see the cohesiveness of the } \\
\text { nurses on NICU } 3\end{array}$ & $\begin{array}{l}\text { Physical space } \\
\text { - Individual rooms } \\
\text {-Central monitoring }\end{array}$ & $\begin{array}{l}\text { Learn the baby } \\
\text {--routine care } \\
\text {--comfort care }\end{array}$ \\
\hline \multicolumn{3}{|l|}{ 9. Are there any barriers to providing care? } \\
\hline 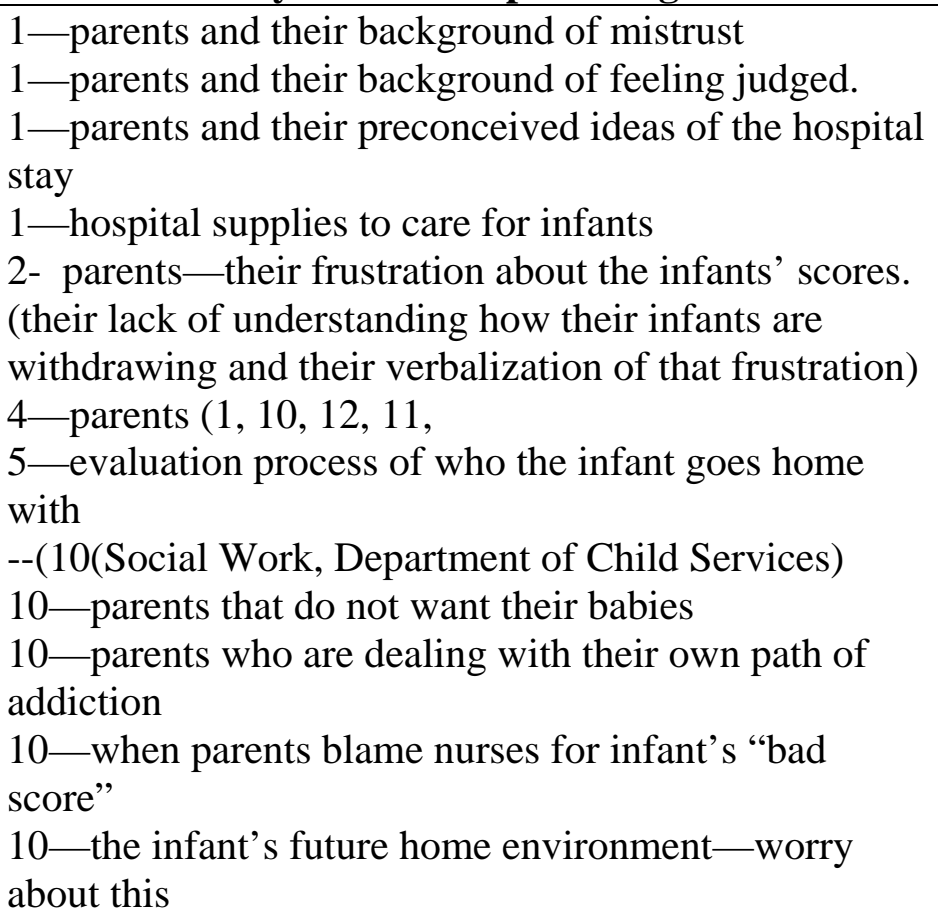 & $\begin{array}{l}\text { Parents } \\
\text {-Background of mistrust } \\
\text {-Background of feeling judged } \\
\text {-Background of addiction } \\
\text {-Background of blame } \\
\text {-Misunderstanding of NAS } \\
\text { care } \\
\text {-Intoxicated parents and safety } \\
\text { of infant } \\
\text {-Unit rules for parents } \\
\text { Evaluation Process for the } \\
\text { infant's future home } \\
\text { Core team helps decrease } \\
\text { barriers: } \\
\text {--Primary care, } \\
\text {--family-centered care } \\
\text {--what baby needs }\end{array}$ & $\begin{array}{l}\text { Grief } \\
\text { Core team support }\end{array}$ \\
\hline
\end{tabular}




\begin{tabular}{|c|c|c|}
\hline 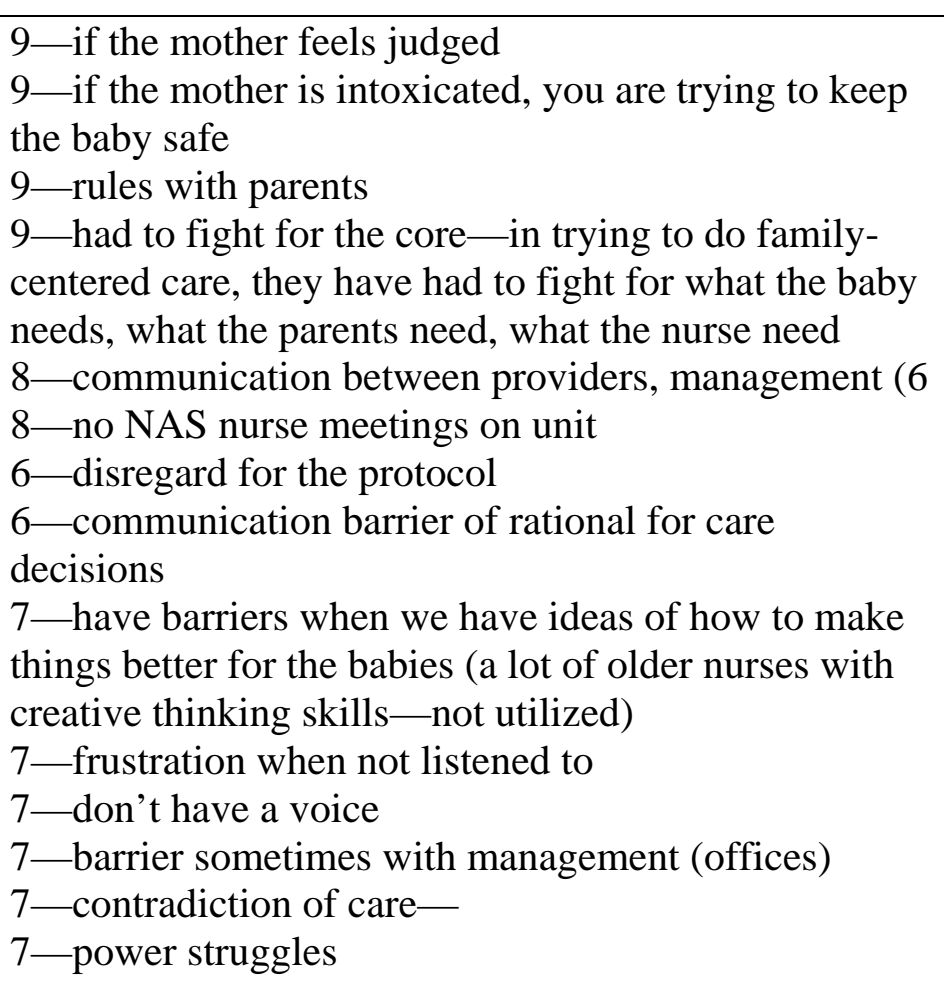 & $\begin{array}{l}\text { Communication } \\
\text {--NAS meetings on unit with } \\
\text { nurses } \\
\text {--Changes to the protocol } \\
\text {--Rationale for decisions } \\
\text {--Ideas to help babies } \\
\text {--Frustration when not listened } \\
\text { to } \\
\text {--Power struggles } \\
\text {-- between healthcare personnel }\end{array}$ & $\begin{array}{l}\text { Communication barriers (for the end- } \\
\text { future recommendations) }\end{array}$ \\
\hline \multicolumn{3}{|l|}{ 10. How do you cope with the difficult days? } \\
\hline $\begin{array}{l}\text { 2-cope by talking and joking, keep "going about } \\
\text { your day” (6, FN, } \\
\text { 4-deep breathing } \\
4 \text { - coping mechanisms improve over time-learn to } \\
\text { cope with the noise, stress, figure out your priorities } \\
\text { for care } \\
5 \text {-remembering that the baby will get better as they } \\
\text { withdraw } \\
\text { 5-looking forward to normal baby behavior }\end{array}$ & $\begin{array}{l}\text { Coping mechanisms } \\
\text {-Talking } \\
\text {-Joking/teasing } \\
\text {-Keep going } \\
\text {-Figuring out priorities in care } \\
\text {-Remembering the baby will } \\
\text { get better } \\
\text {-Focus on what you can do } \\
\text {-Thankful for enough help } \\
\end{array}$ & Core Team Support \\
\hline
\end{tabular}




\begin{tabular}{|c|c|c|}
\hline 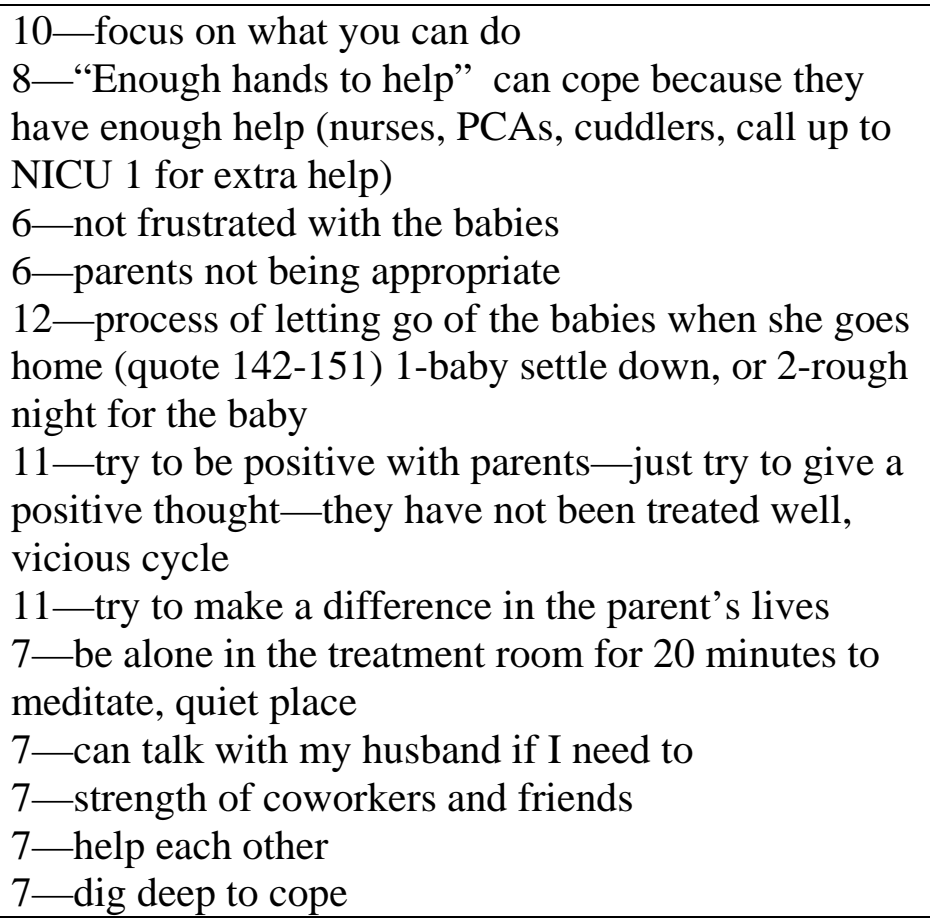 & $\begin{array}{l}\text {-Letting go of the babies } \\
\text {-family support } \\
\text {-Quiet place on unit to meditate } \\
\text {-inner strength } \\
\text {-Help each other cope and let } \\
\text { them help you }\end{array}$ & \\
\hline $\begin{array}{l}\text { 11. What are the successes you experience in } \\
\text { working with these infants? }\end{array}$ & & \\
\hline $\begin{array}{l}\text { 1- education of mothers about how to improve their } \\
\text { lives (6-breastfeeding education, } 7 \text {, } \\
\text { 1-giving away knowledge to mothers } \\
\text { 2-I feel good about giving good care to the } \\
\text { infants....so much that they are spoiled in the end } \\
\text { because of so much love. } \\
\text { 4-any time that you get appreciation from the family } \\
\text { for your care of the baby } \\
\text { 5-when they are well enough to go home } \\
\text { 5-when babies have good moments } \\
\text { 10-when an infant overcomes their beginning and } \\
\text { becomes more normal (5, }\end{array}$ & $\begin{array}{l}\text { Babies overcoming their } \\
\text { beginning } \\
\text {-Begin to act normal } \\
\text {-Progression from mistrust to } \\
\text { trust } \\
\text {--well enough to go home }\end{array}$ & Making a difference \\
\hline
\end{tabular}




\begin{tabular}{|c|c|c|}
\hline  & $\begin{array}{l}\text {--knowing the babies } \\
\text {--the babies know you } \\
\text { Helping parents } \\
\text { - Education } \\
\text {-Breastfeeding education }\end{array}$ & Making a difference \\
\hline $\begin{array}{l}\text { 12. What are the challenges/hardships to working } \\
\text { with these infants? }\end{array}$ & & \\
\hline $\begin{array}{l}\text { 2-infant withdrawal symptoms (12, } \\
4 \text {-inconsistency of how infants progress and } \\
\text { wean...hard to watch this. } \\
\text { 5-unknown future at home }(10,9,12,7 \text {-death, } \\
10 \text { - parents }(6,7 \\
10 \text { - wondering if your work was for nothing } \\
8 \text {-a lot of barriers in educating the parents/family } \\
\text { 8- no psych training for nurses }\end{array}$ & $\begin{array}{l}\text { Infant's withdrawal } \\
\text {-Extreme fussiness } \\
\text {-Inconsistency of withdrawal } \\
\text {-Emotional strain on nurses } \\
\text {-Empathy of nurses for infant's } \\
\text { pain } \\
\text { Infant's future }\end{array}$ & $\begin{array}{l}\text { Making a difference } \\
\text { Grief }\end{array}$ \\
\hline
\end{tabular}




\begin{tabular}{|c|c|c|}
\hline $\begin{array}{l}\text { 6-challenge of education of the parents, but } \\
\text { hopefully plant seeds, impact families } \\
11 \text {-DCS-broken system, not sure of their guidelines } \\
\text { for the infant to go home with parents (7, } \\
7 \text { - I am supposed to be their advocate, but I am not } \\
\text { heard. } \\
\text { 7--management }\end{array}$ & $\begin{array}{l}\text {-All in vain? } \\
\text {-Home environment-possibly } \\
\text { unstable } \\
\text {-Education of parents } \\
\text {-DCS and decisions they make } \\
\text {-Fear of infant's death at home } \\
\\
\text { Infant's advocate } \\
\text {-Nurses not heard } \\
\text {-not understanding the process }\end{array}$ & Personal role satisfaction \\
\hline \multicolumn{3}{|l|}{ 13. What makes work easier for you? } \\
\hline 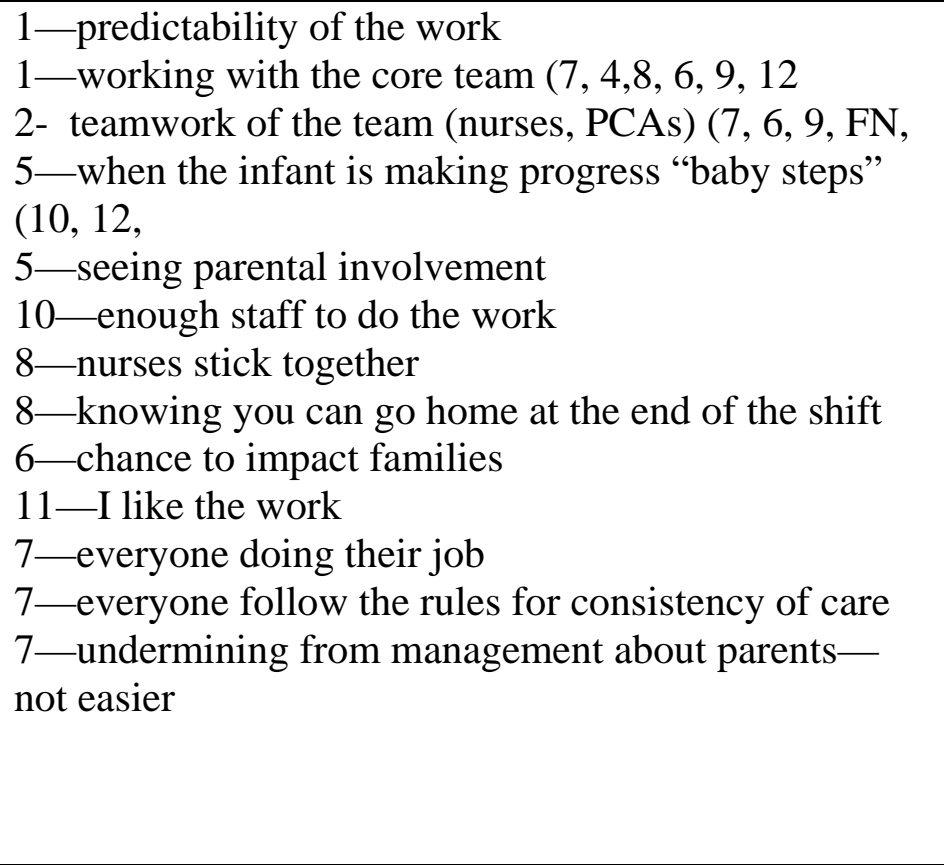 & $\begin{array}{l}\text { Teamwork of Core Team } \\
\text {-Teamwork } \\
\text {-There for each other } \\
\text {-Support system } \\
\text {-Friendships } \\
\text {-Enjoyable work } \\
\text {-Doing their job } \\
\\
\text { Infant doing better } \\
\text {-Babies comfortable } \\
\text {-Making progress } \\
\\
\text { Parents doing better } \\
\text {-Parental involvement in } \\
\text { infant’s care } \\
\text {-Family impacted }\end{array}$ & Making a difference \\
\hline $\begin{array}{l}\text { 14. What is it about this unit in particular that } \\
\text { makes you want to work here? }\end{array}$ & & \\
\hline
\end{tabular}




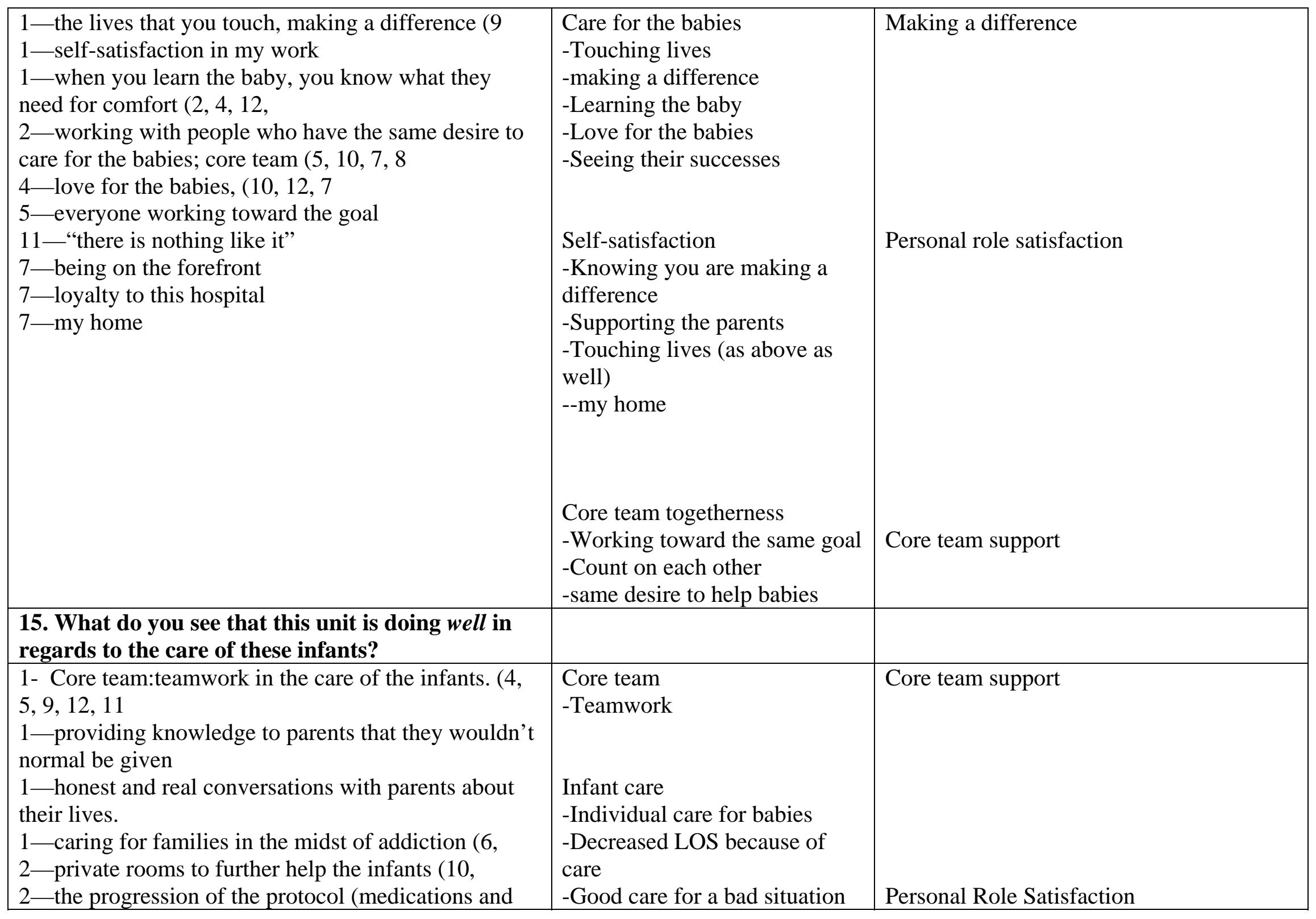




\begin{tabular}{|c|c|c|}
\hline 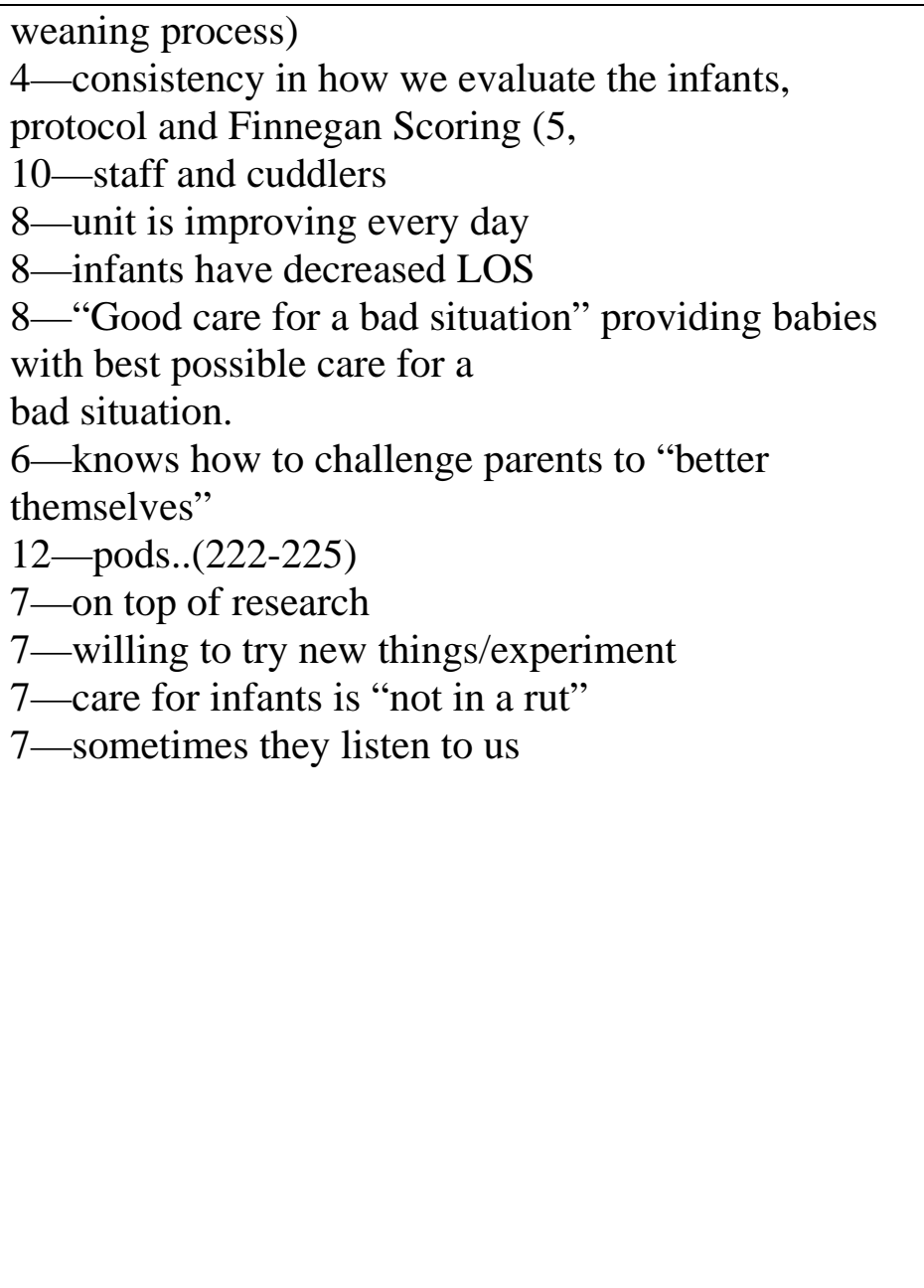 & $\begin{array}{l}\text {-consistent care } \\
\text { In the midst of addiction } \\
\text {-Providing knowledge } \\
\text {-Engaging with families } \\
\text {-Know how to challenge } \\
\text { parents } \\
\text {-Honesty with parents } \\
\text {-Kindness to families } \\
\text {-Caring for them } \\
\text { Physical space } \\
\text {-Own room, crib, swing, } \\
\text { parents care for baby in room } \\
\text {-Pods of nursing care (1,2,3; } \\
\text { 4,5,6) } \\
\text { Caregivers/Practitioners } \\
\text { Progressiveness } \\
\text {-On top of research } \\
\text {-Willing to try new things } \\
\text {-Care for infants is “not in a } \\
\text { rut” } \\
\text {-Sometimes nurses are listened } \\
\text { to }\end{array}$ & Learn the baby \\
\hline \multicolumn{3}{|l|}{$\begin{array}{l}\text { 16. What do you see that this unit needs to improve } \\
\text { on in regards to the care of these infants? }\end{array}$} \\
\hline $\begin{array}{l}\text { 1-for management to understand the benefits of a } \\
\text { core team for the care of the babies and the trust of the } \\
\text { parents } \\
1-326-327 \text { quote...great satisfaction when parents }\end{array}$ & $\begin{array}{l}\text { Communication and } \\
\text { collaboration } \\
\text {-All disciplines } \\
\text {-Process of how and who a }\end{array}$ & $\begin{array}{l}\text { Communication-for future } \\
\text { recommendations }\end{array}$ \\
\hline
\end{tabular}




\begin{tabular}{|c|c|c|}
\hline 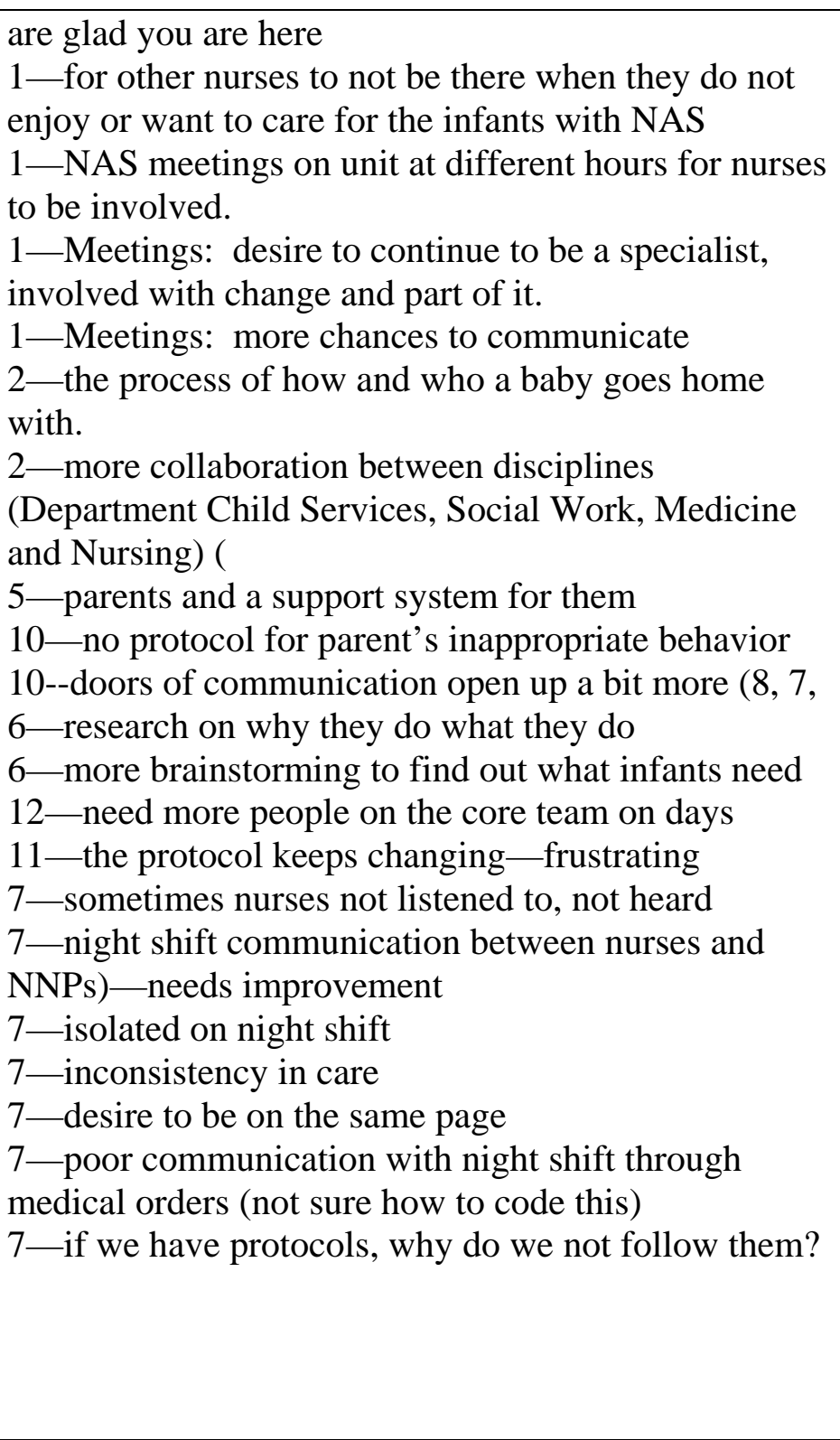 & $\begin{array}{l}\text { baby goes home with } \\
\text {-Meetings on the unit for the } \\
\text { nurses } \\
\text {-Changes on the unit } \\
\text { (protocols) } \\
\text {-Night shift not heard } \\
\text {-Night shift isolated from } \\
\text { NNPs } \\
\text { Appreciate the Core Team } \\
\text {--outside appreciation for core } \\
\text { team and the care for the babies } \\
\text {--need more on core team } \\
\\
\text { Parents } \\
\text {--support system for them } \\
\text {--protocols for inappropriate } \\
\text { behavior } \\
\text {--consequences }\end{array}$ & Making a difference \\
\hline
\end{tabular}




\begin{tabular}{|c|c|c|}
\hline & protocols & \\
\hline $\begin{array}{l}\text { Thank you for sharing your insights on the NICU } \\
\text { environment and the care of these infants. I would } \\
\text { like to switch gears for a moment and ask you some } \\
\text { more specific questions regarding some of the } \\
\text { interventions } \\
\text { 1. Are there types of interventions that you have } \\
\text { found helpful in soothing these infants? (Then go } \\
\text { into your specific questions). }\end{array}$ & & \\
\hline $\begin{array}{l}\text { 1-bathing (10, 12, 11, 7, FN, 9, 12) } \\
\text { 1-weighted blankets or frogs } \\
\text { 1-Halo swaddle, wrapping with hands midline (FN, } \\
11 \\
\text { 2-swaddling (4, 5, 10, 9, 8, 6, FN, 12, 11) } \\
\text { 2-music boxes (5, 10, 9, FN) } \\
\text { 2- swing (4, 10, 8, FN, 11, 8) } \\
\text { 2-mobile (4, 10, FN) } \\
\text { 2-held and rocked (4, 5, 10, 6, 12, 11, FN) } \\
\text { 4-learn what works. } \\
\text { 4-Tangled in leads (FN } \\
\text { 4- pacifier (FN } \\
\text { 4-change diaper (10, FN } \\
\text { 4-holding (5, 10, 6, FN, } 12 \\
\text { 4-walking, FN } \\
\text { 4-stroller (10, FN } \\
\text { 5-quiet (FN } \\
\text { 10-pacifier (6, FN, 2, } 6 \\
\text { 10- dark room (FN } \\
\text { 10-cuddler available hold baby (FN } \\
\text { 10-talk to them/sing (FN, } \\
\text { 10-patting on the bottom (FN, 12, }\end{array}$ & $\begin{array}{l}\text { Comfort Measures } \\
\text { Baths } \\
\text { Weighted frogs/blankets } \\
\text { Swaddling } \\
\text { Music boxes } \\
\text { Swings } \\
\text { Mobiles } \\
\text { Held and rocked } \\
\text { Untangled leads and pulse ox } \\
\quad \text { Cords } \\
\text { Walking with } \\
\text { Talking with } \\
\text { Stroller } \\
\text { Quiet room } \\
\text { Pacifier } \\
\text { Diaper change } \\
\text { Dark room } \\
\text { Patting on the bottom } \\
\text { White noise } \\
\text { Swaddle with hands in } \\
\text { Bouncing } \\
\text { Rhythmic movement }\end{array}$ & $\begin{array}{l}\text { Learn the baby } \\
\text {--comfort care }\end{array}$ \\
\hline
\end{tabular}




\begin{tabular}{|c|c|c|}
\hline $\begin{array}{l}\text { 9-white noise (FN } \\
\text { 8--Sweet Ease(only occasionally use) (FN } \\
\text { 6-rhythmic bouncing movement (FN } \\
\text { 6--swaying with infant (FN } \\
\text { 6--breastfeeding hold (FN } \\
\text { FN-- front backpack carrier, older infants held in the } \\
\text { nurses station-talking to and interacting with baby, } \\
\text { 12-belly across nurses legs, } \\
\text { 11--heel warmers on belly (FN, 10, } \\
\text { 11-barrier cream (desitin and cornstarch) and barrier } \\
\text { spray for their sore bottoms } \\
\text { 11-Trial and error, individual } \\
\text { 7--lotion massage on tummy } \\
\text { 7--warm their formula } \\
\text { 7-keep room cool }\end{array}$ & $\begin{array}{l}\text { Swaying with infant } \\
\text { Hold tight, pacifier in mouth, } \\
\text { breastfeeding position } \\
\text { talking to baby } \\
\text { singing to baby } \\
\text { heel warmer to abdomen } \\
\text { Sweet-ease if very fussy } \\
\text { Front pack carrier } \\
\text { Older infants-in nurse’s } \\
\text { station and interacting with } \\
\text { baby } \\
\text { tummy to tummy with nurse } \\
\text { belly across your legs } \\
\text { Halo swaddle } \\
\text { Barrier cream and spray } \\
\text { Sound machines (storm) } \\
\text { Lotion massage while on } \\
\text { tummy. } \\
\text { Warm the formula } \\
\text { Room cool }\end{array}$ & \\
\hline $\begin{array}{l}\text { 2. I have noticed that all infants are on a } 3 \text { hour } \\
\text { feeding schedule, could you please describe how } \\
\text { you care for infants every } 3 \text { hours... what are the } \\
\text { nursing interventions that you do? }\end{array}$ & & \\
\hline $\begin{array}{l}\text { 1-full assessment, b/p, temp, hr, resp., diaper change, } \\
\text { feed }(2,4,5,8,9,6, \mathrm{FN}, 12,11,7,10 \\
1 \text { - nights: weight and bath }(11,7) \\
1 \text {-after feeding, try to start lying the infant in the crib } \\
1 \text { - } \text {-stock room with supplies }(9,11,6)\end{array}$ & Routine care & $\begin{array}{l}\text { Learn the Baby } \\
\text {--Routine care }\end{array}$ \\
\hline
\end{tabular}




\begin{tabular}{|c|c|c|}
\hline $\begin{array}{l}\text { 2->28 days, may feed and possibly sleep q. 3-4 hours. } \\
\text { 4-change pulse ox to the other foot, change id band } \\
\text { to the other leg }(10,11) \\
10 \text { - (quote } 276-282) \text {. } \\
\text { 6--when older, stroller and walk around the unit, in } \\
\text { nurse's station with people around } \\
\text { 7- warm feeding (8) } \\
\text { 7--feed for 30, hold for 30, } \\
\text { 7- swings-last resort }\end{array}$ & & \\
\hline $\begin{array}{l}\text { 3. After this every } 3 \text { hour care, what are the } \\
\text { nursing strategies you use to help infants with NAS } \\
\text { to sleep or be comforted between feedings? }\end{array}$ & & \\
\hline $\begin{array}{l}\text { 1-not full assessment, resp, heart rate, temp. (10, 4, 5, } \\
\text { FN } \\
\text { 1-swaddling (4, 5, 10, FN, 12, } \\
\text { 1--midline hands and tight swaddling, FN } \\
\text { 1-change diaper (5, 10, 12, FN } \\
\text { 1-close holding to keep the infant midline (2, FN } \\
\text { 1- room dark to decrease simulation (FN, 11, } \\
\text { 4-Finnegan scoring with your pod of } 3 \text { rooms } \\
\text { 4-holding, rocking (FN, 11, 7, } \\
\text { 5-be around in your pod area and hear babies, 5- } \\
\text { pacifier (FN, 12, 7, 11, 10, } \\
\text { 5--touch them or pat them (FN, 7, 8, } \\
\text { 10-temp, v.s. from monitor, change diaper and feed, } \\
\text { chart, } \\
\text { 10--talk with babies (FN, } \\
\text { 10--talk with parents, } \\
\text { 8-checking leads and pulse ox probes on fussy } \\
\text { infants with alarms going off, } \\
\text { 8- swing (FN, 12, 7, } \\
\text { 8-stroller (FN } \\
\text { 8--need stimulation or not, >28 days-more awake }\end{array}$ & Comfort care & $\begin{array}{l}\text { Learn the baby } \\
\text {--comfort care }\end{array}$ \\
\hline
\end{tabular}




\begin{tabular}{|c|c|c|}
\hline $\begin{array}{l}\text { (10, } \\
8 \text {-child life may come to play with infant when } \\
\text { awake } \\
\text { FN_-tummy to tummy with nurse } \\
\text { FN-quiet room } \\
\text { FN_-Sweet Ease if very fussy (10, } \\
\text { FN_-front backpack holder or Moby wrap (2, } \\
\text { FN-Halo swaddle } \\
\text { 12-unwrapped in monitor cords (FN } \\
\text { 12- cuddlers hold babies (FN, } \\
\text { 7-lighted mobiles (FN, } \\
\text { 7-sound machine (FN, }\end{array}$ & & \\
\hline $\begin{array}{l}\text { Specific Nursing Intervention Questions: } \\
\text { 1. What effect does light, noise and touch have on } \\
\text { NAS infants? What do you do to control light, } \\
\text { noise and touch? (Environment) }\end{array}$ & & \\
\hline $\begin{array}{l}\text { (organize; place different responses for each item next } \\
\text { to each other, e.g., light, sound, etc.) } \\
\text { Light: } \\
\text { 1-room dark to decrease stimulation, will eat better } \\
\text { (2, 5, 9, 8, 6, 12, 11, 7, 9, 8, FN, 12, 11, } 5 \\
\text { 2--some nurses try to keep the day light during the } \\
\text { day, and dark in the room at night (7, FN, 12, 11,7, } 5 \\
\text { Noise: } \\
\text { 2- sensitive to noise (5, 9, 6, 8, FN, 7, 12, 7, } 5 \\
\text { 4-like white noise (5, 9, 6, 8, FN, 12, 11, 7, 5, } \\
\text { Touch: } \\
\text { 4--hypersenstitive--some do not like to be touched } \\
\text { (9, 8, FN, } 12,11,7\end{array}$ & $\begin{array}{l}\text { Before captured } \\
\text {--Hypersensitive to light, noise } \\
\text { and touch } \\
\text { Light: } \\
\text {--keep room dark } \\
\text {-- Some do not seem to mind } \\
\text { light } \\
\text {-- Small amounts of light (day } \\
\text { time) } \\
\text {--some feed better in dark } \\
\text { Sound: } \\
\text {--quiet or white noise } \\
\text {--some do not mind } \\
\text {--shift change noise }\end{array}$ & $\begin{array}{l}\text { Learn the baby } \\
\text {--environment }\end{array}$ \\
\hline
\end{tabular}




\begin{tabular}{|c|c|c|}
\hline $\begin{array}{l}\text { 5-need to treat babies like they have a } \\
300 \% \text { migraine. Private rooms help with this decrease } \\
\text { in stimulation } \\
\text { 10-depends on if uncaptured (quote } 307-317 \text { ). } \\
12 \text { - depends on the baby. }\end{array}$ & $\begin{array}{l}\text { Touch: } \\
\text {--gentle touch, patting on } \\
\text { bottom, rhythmic, soft, -- } \\
\text { nurturing touch } \\
\text {--some "touch me nots" } \\
\text { Depends on the baby } \\
\text {--some it matters and with } \\
\text { others it does not } \\
\text { Day and night schedules }\end{array}$ & \\
\hline \multicolumn{3}{|l|}{$\begin{array}{l}\text { 2. Describe how you help an infant with NAS to get } \\
\text { adequate sleep and rest? (Adequate rest and sleep) }\end{array}$} \\
\hline $\begin{array}{l}\text { 1-comfort measures help them sleep } \\
\text { 1-knowing the baby helps you to provide the right } \\
\text { comfort for the baby } \\
\text { 2--swaddle (4, 5, 10, 6, FN, 12, } \\
\text { 2--pacifier (8, FN, } \\
\text { 2--swing (try to limit swing because many do not have } \\
\text { swings at home), try crib first, (4, 5, 10, FN, } \\
\text { 2--holding infant (4, 5, 8, FN, } \\
\text { 5--lights out, FN, 12, 11, } \\
\text { 5-music, sound machine (FN, 12, } \\
\text { 5- quiet (FN, } \\
\text { 10-cool room (FN } \\
\text { 10-rocking (8, FN } \\
\text { 9--keep environmental stimuli to a minimum, } 11 \\
\text { 12-trial and error for what works. } \\
\text { 7- different schedule for day and night } \\
\text { 7- promote a home setting for rest }\end{array}$ & $\begin{array}{l}\text { Comfort measures } \\
\text { Keep environmental stimuli } \\
\text { decreased } \\
\text { Promote a home setting for } \\
\text { sleep and rest } \\
\text { Trial and error } \\
\text {--learn the baby } \\
\text { Day and night rhythms }\end{array}$ & $\begin{array}{l}\text { Learn the baby } \\
\text {--adequate rest and } \\
\text { Learn the baby }\end{array}$ \\
\hline 3. How would you describe feeding an infant with & & \\
\hline
\end{tabular}




\begin{tabular}{|c|c|c|}
\hline NAS (formula or breast milk)? (Feeding) & & \\
\hline 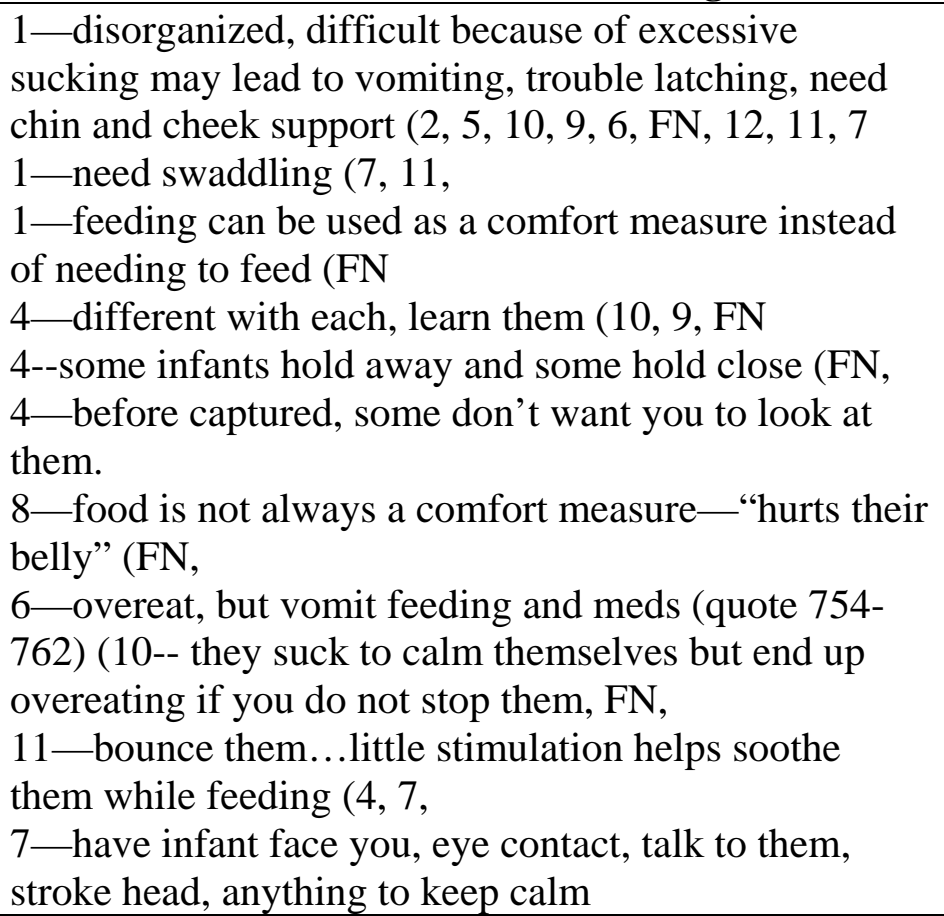 & $\begin{array}{l}\text { Help with feeding } \\
\text {--trouble latching } \\
\text {--positioning (close or away) } \\
\text {--chin and cheek support } \\
\text {-individual differences } \\
\text {-swaddle/not swaddle } \\
\text {--bounce some } \\
\text {--eye contact/no eye contact } \\
\text { Food is comfort } \\
\text {---don't overfeed (vomiting) } \\
\text { Food is not comfort } \\
\text {--hurts belly }\end{array}$ & $\begin{array}{l}\text { Learn the baby } \\
\text {--feeding }\end{array}$ \\
\hline $\begin{array}{l}\text { 4. How would you describe Finnegan scoring? } \\
\text { (Assessment and evaluation using Finnegan } \\
\text { scoring) }\end{array}$ & & \\
\hline $\begin{array}{l}\text { 1-Core team: knowing/learning the baby leads to } \\
\text { accurate scoring (10, 8, FN, } 7 \\
1 \text {-when scoring, if you know the baby, you can } \\
\text { differentiate between withdrawal symptoms and the } \\
\text { personality of the infant } \\
2 \text {-complex: symptoms are assigned numbers, but } \\
\text { subjective (10,9, FN, 7, 12-2 things: clinical part } \\
\text { (“the thing that if it's there, it's there.”) and the } \\
\text { subjective part- depends on the person doing the } \\
\text { scoring. }\end{array}$ & $\begin{array}{l}\text { Knowing the baby leads to } \\
\text { accurate scoring } \\
\text {-- Differentiate between } \\
\text { withdrawal scores and the } \\
\text { personality of the baby } \\
\text {--core team consistency } \\
\text {--core team accuracy } \\
\text { After captured }\end{array}$ & $\begin{array}{l}\text { This I will discuss differently than the } \\
\text { other } 4 \text { nursing interventions }\end{array}$ \\
\hline
\end{tabular}




\begin{tabular}{|c|c|c|}
\hline $\begin{array}{l}\text { 4> } 28 \text { days not score for sleep (6, FN } \\
\text { 5- scoring system with the goal to keep infants below } \\
\text { 8, } \\
\text { 9-“art to it” Need to understand the symptoms, 9- } \\
\text { example of excoriation (quote 429-438) } \\
\text { 8--best tool out there } \\
\text { 8- -scoring is specific } \\
\text { 8--better with practice and education } \\
\text { 8-early on-in-services on Finnegan scoring } \\
\text { 6-good for the first week } \\
\text { 11-1 hour after meds given } \\
\text { 11-scoring the infant's symptoms of withdrawal and } \\
\text { tells you how much medication needs to go up or stay } \\
\text { where it is. } \\
\text { 7-can be scored high for things that can be controlled }\end{array}$ & $\begin{array}{l}\text {-- >28 days, no score for sleep } \\
\text { Complex } \\
\text {--subjective at times } \\
\text {--objective } \\
\text {--specific symptoms } \\
\text {--can be manipulated } \\
\text {--best tool out there } \\
\text {--requires education and } \\
\text { practice }\end{array}$ & \\
\hline $\begin{array}{l}\text { 5. What role do you see yourself in with these } \\
\text { infants?” (Nurses like mothers to infants) }\end{array}$ & & \\
\hline 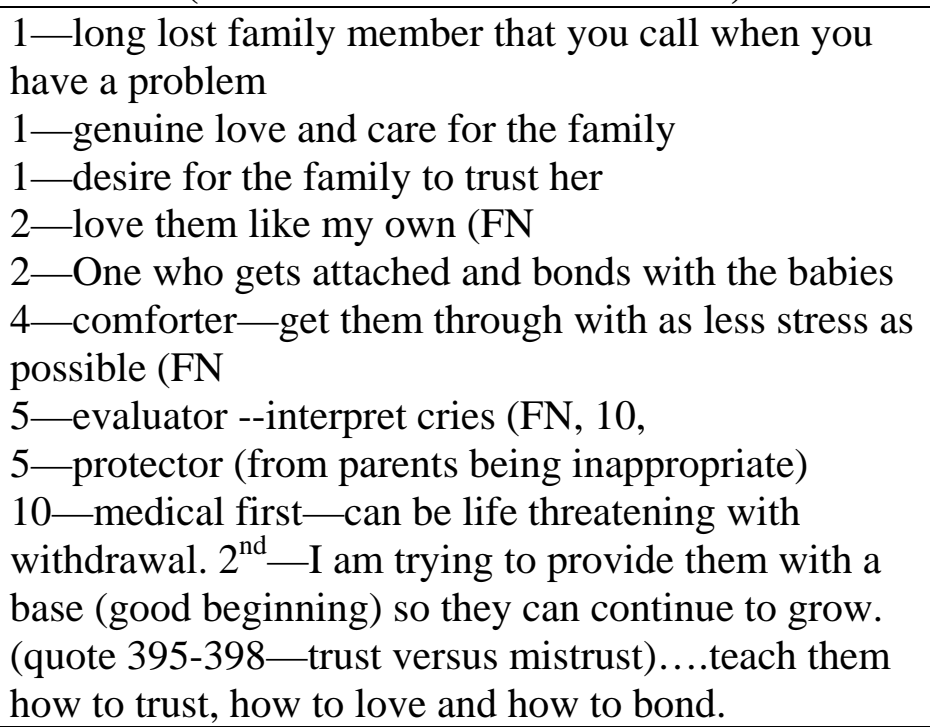 & $\begin{array}{l}\text { Comforter } \\
\text {--Nurturer } \\
\text {--Genuine love for family } \\
\text {-- Long lost family member } \\
\text {--attach and bond with baby } \\
\text { Protector } \\
\text { Advocate } \\
\text { Support system } \\
\text { Caretaker }\end{array}$ & $\begin{array}{l}\text { Personal role satisfaction } \\
\text {--nurturer/comforter } \\
\text { Personal role satisfaction }\end{array}$ \\
\hline
\end{tabular}




\begin{tabular}{|c|c|c|}
\hline 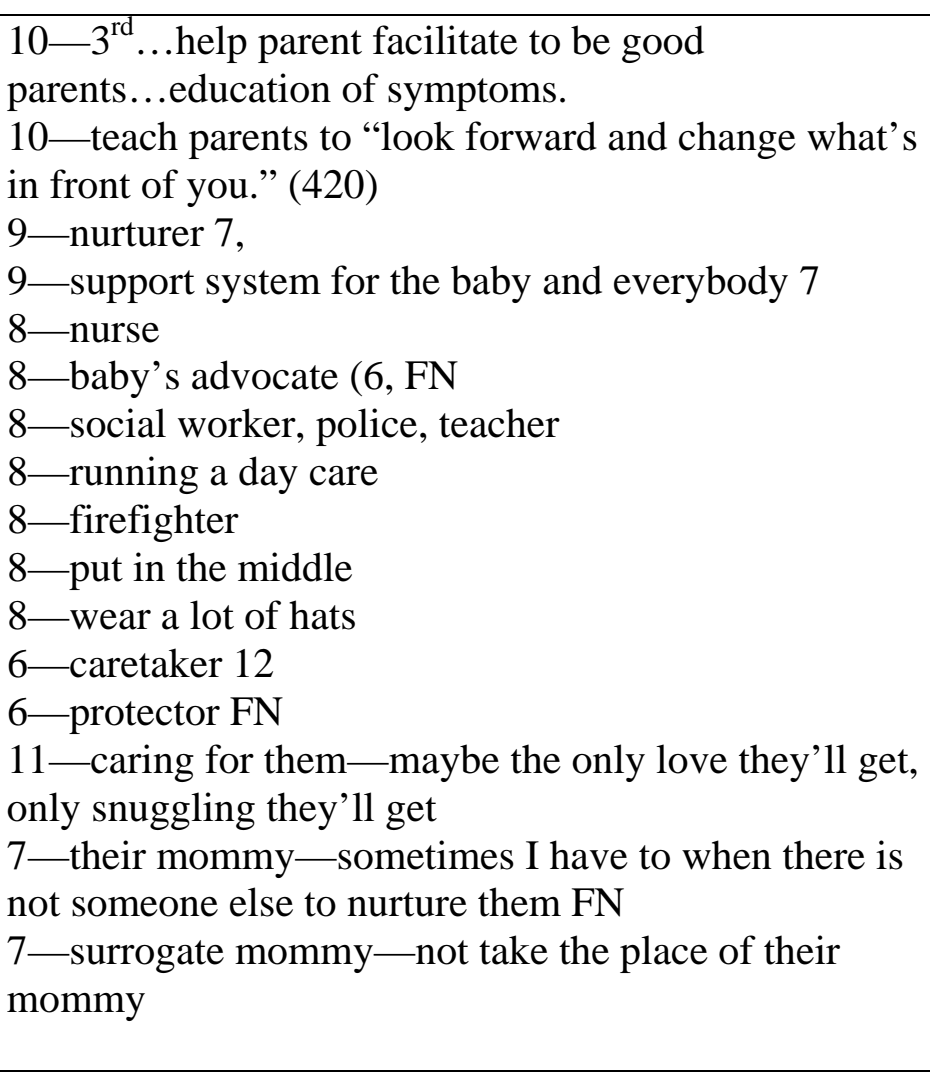 & $\begin{array}{l}\text { Nurse } \\
\text { Medical } \\
\text { Good start } \\
\text { Educator } \\
\text { In the middle } \\
\text { Multiple hats } \\
\text {--firefighter } \\
\text {--social worker } \\
\text {--police } \\
\text {--running a daycare } \\
\text { Surrogate mommy } \\
\text {--not take place of mommy }\end{array}$ & $\begin{array}{l}\text { Personal role satisfaction } \\
\text {--nurturer or comforter }\end{array}$ \\
\hline $\begin{array}{l}\text { Last few questions: } \\
\text { What successes do you personally experience?(you } \\
\text { may want to modify this question for the purpose } \\
\text { of writing.) }\end{array}$ & & \\
\hline $\begin{array}{l}\text { 2-when infants respond to your care }(4,9,6,7,11 \text {, } \\
\text { 2-when infants get better }(4,5,7,12,6 \\
\text { 4-no regrets in working here } \\
\text { 10-when a baby goes home to a good home, when } \\
\text { you feel like a baby has a real chance }(5,7, \\
\text { 9-reward of working with the babies } \\
\text { 8-inner and personal success in caring for the babies }\end{array}$ & $\begin{array}{l}\text { Making a difference } \\
\text {--Babies get better (relax, heal) } \\
\text {--Baby going to a good home } \\
\text {--Education of family } \\
\text {--feeling and knowing } \\
\text { Inner successes }\end{array}$ & Making a difference \\
\hline
\end{tabular}




\begin{tabular}{|c|c|c|}
\hline $\begin{array}{l}\text { 6-educate a mom to being more competent } \\
\text { 6- help mom to stick with caring for the baby }\end{array}$ & $\begin{array}{l}\text {-respond to your care } \\
\text {--helping the baby } \\
\text {--helping the mom } \\
\text {--no regrets }\end{array}$ & Personal role satisfaction \\
\hline \multicolumn{3}{|l|}{ What hardships do you personally experience? } \\
\hline 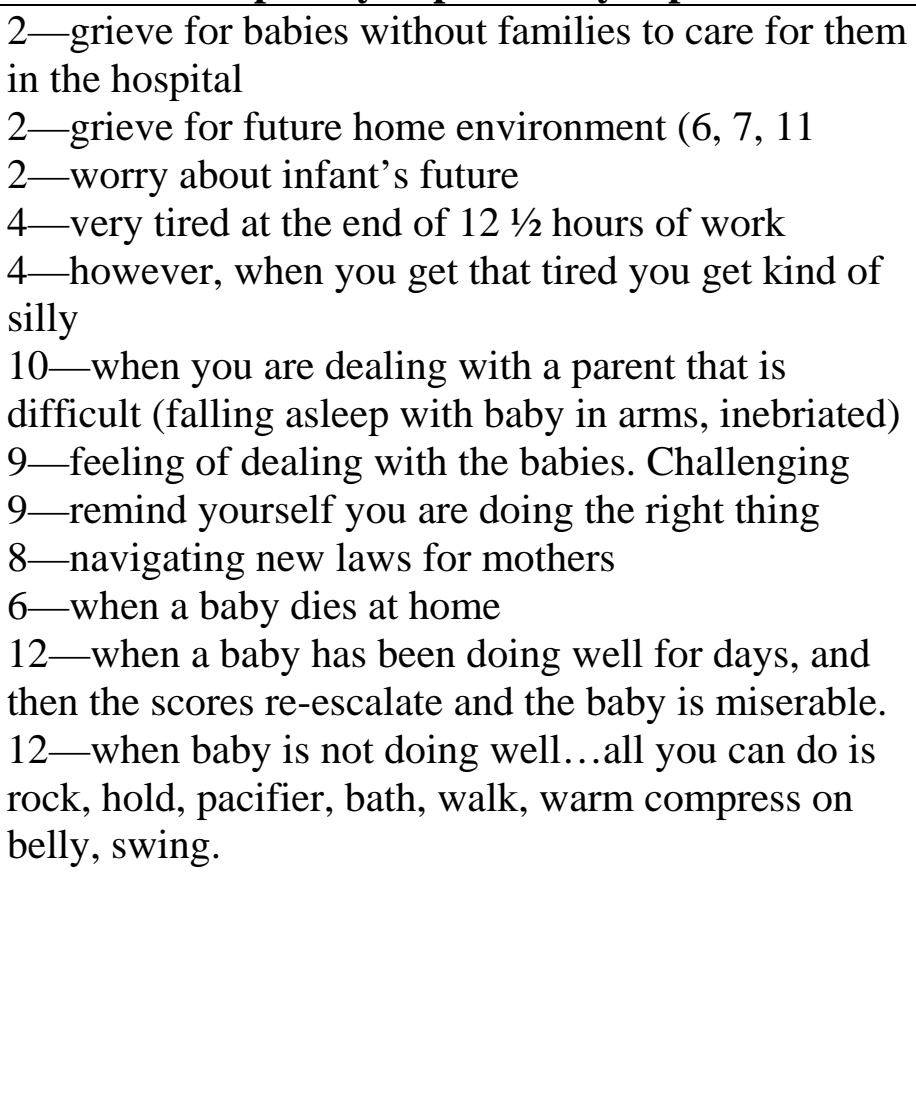 & $\begin{array}{l}\text { Grief for today } \\
\text {--When families not present to } \\
\text { care for infants } \\
\text {--When a baby is not doing } \\
\text { well, or re-escalates } \\
\\
\text { Grief for the future } \\
\text {--Future home environment } \\
\text {--New laws for mothers } \\
\text {--Reported deaths after } \\
\text { discharge } \\
\text {--Powerlessness to change the } \\
\text { future } \\
\text { Tiredness and stress } \\
\text {--Stress of the job } \\
\text {--Stress of parents } \\
\text {--Stress of babies }\end{array}$ & Grief \\
\hline $\begin{array}{l}\text { Is there anything else about your experiences on } \\
\text { NICU } 3 \text { that you have not had the chance to } \\
\text { express that you feel is important? (Be } \\
\text { comfortable with silence to give people time to } \\
\text { generate their thoughts) }\end{array}$ & & \\
\hline
\end{tabular}




\begin{tabular}{|c|c|c|}
\hline 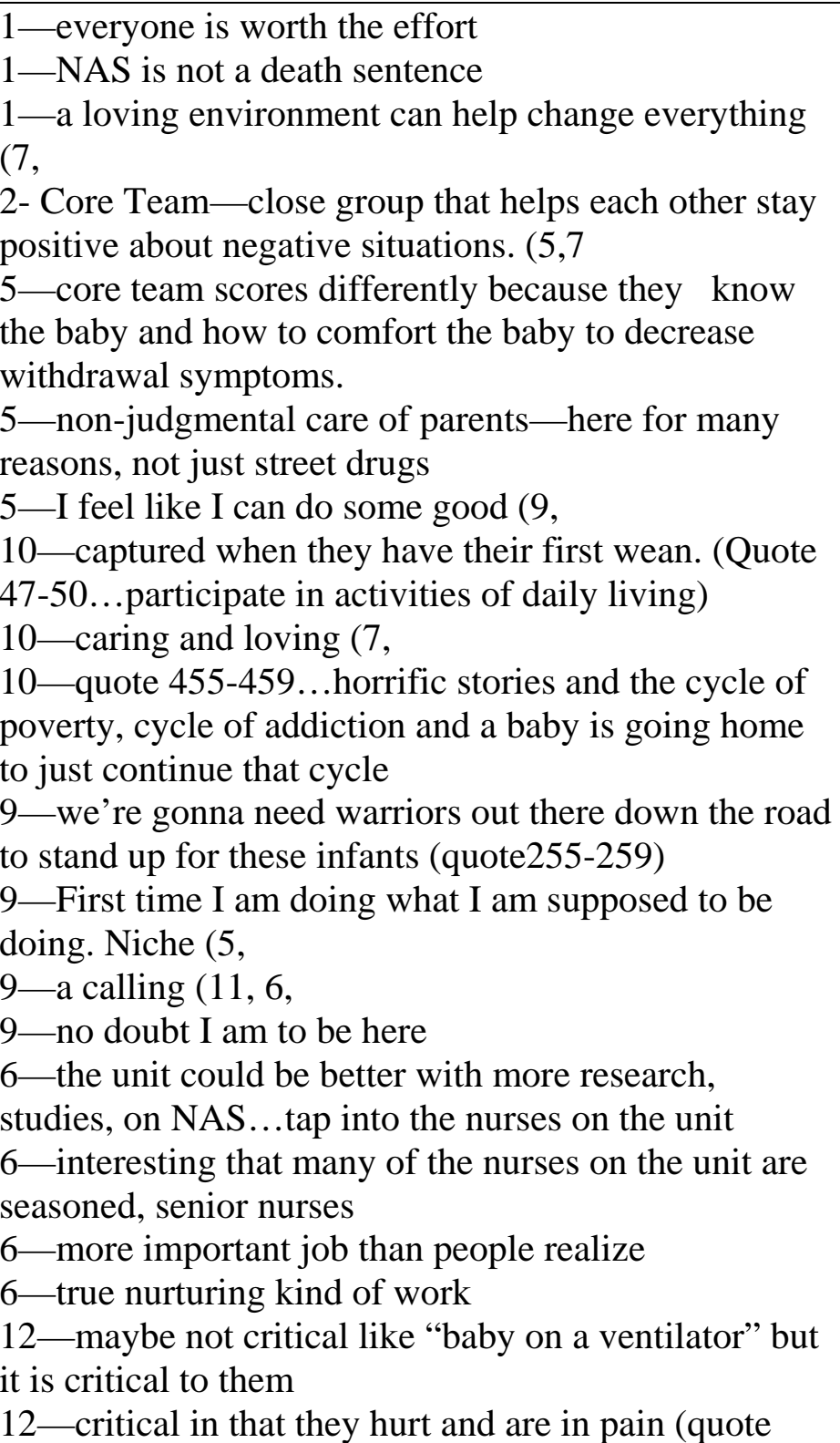 & $\begin{array}{l}\text { Worth the effort } \\
\text {--A loving environment can } \\
\text { make a difference } \\
\text {--Warriors who stand up for } \\
\text { these infants } \\
\text {--More important than people } \\
\text { realize } \\
\text {--Nurturing work } \\
\text { Core team } \\
\text {--Close group of nurses who } \\
\text { care for babies } \\
\text {--A calling for them } \\
\text {--Other nurses do not think this } \\
\text { is nursing-overcoming this } \\
\text { stigma } \\
\text {-- "Positive about the negative” } \\
\text {--Non-judgmental care of } \\
\text { parents }\end{array}$ & $\begin{array}{l}\text { Making a difference } \\
\text { Personal Role satisfaction } \\
\text {--nurture/comforter }\end{array}$ \\
\hline
\end{tabular}




\begin{tabular}{|c|c|c|}
\hline $\begin{array}{l}\text { 432-434... what we do is critical with them and you } \\
\text { know it makes a difference as to what A loving } \\
\text { environment can make a difference } \\
\text { their day's gonna be like.” } \\
\text { 11-unique unit } \\
\text { 11- some say “This is not even nursing” } \\
\text { 7-want to do my job to the best of my ability }\end{array}$ & $\begin{array}{l}\text { "Critical to them" } \\
\text {--baby’s pain } \\
\text {--baby’s hurting } \\
\text {--Other nurses do not think this } \\
\text { is nursing-overcoming this } \\
\text { stigma } \\
\text {--critical in different ways }\end{array}$ & $\begin{array}{l}\text { Making a difference } \\
\text {--Critical to them }\end{array}$ \\
\hline
\end{tabular}




\section{VITA}

\section{MONICA MARIE NELSON}

Education:

Professional Experience:

Publications:
Bachelor of Science in Nursing, Indiana University, Indianapolis, Indiana, 1984

MEd, Lincoln Memorial University, 2010

$\mathrm{PhD}$ Nursing, East Tennessee State University, Johnson City, Tennessee, 2014

King University, 2014 to present

Tennessee Wesleyan College, 2007-2014

Nelson, M. M. (2013). Neonatal abstinence syndrome: The nurse's role. International Journal of Childbirth Education, 28(1), 38-42.

Nelson, M. M. (2013). The benefits of human donor milk for premature infants. International Journal of Childbirth Education, 28(3),84-89.

2012 Award for Professional Nursing Clinical Excellence Sigma Theta Tau International: Epsilon Chapter 